\title{
Sleep-Disordered Breathing in Gestational Hypertension and Preeclampsia: Impact on Maternal and Fetal Outcomes
}

\author{
Submitted by Danielle L. Wilson \\ BSc (Psychology/Psychophysiology), BA (Hons), MSc (Research) \\ ORCID ID - 0000-0001-6754-7359
}

\begin{abstract}
A thesis submitted in total fulfilment of the requirements of the degree of Doctor of Philosophy
\end{abstract} June 2018

School of Medicine - Austin Health Faculty of Medicine, Dentistry and Health Sciences

University of Melbourne

Parkville, Victoria, 3010

Australia 


\begin{abstract}
Introduction: Hypertensive disorders of pregnancy (HDP) include both gestational hypertension $(\mathrm{GH})$ and preeclampsia (PE). They are the most common and serious medical disorders in pregnancy and are a leading contributor to maternal and perinatal morbidity and mortality worldwide. Sleep-disordered breathing (SDB) is reported to occur more commonly in HDP, although the confounding effect of obesity has been variably accounted for. SDB is associated with episodes of hypoxaemia potentially contributing to hypertension through sympathetic activation and oxidative stress, and the activation of inflammatory pathways leading to endothelial dysfunction. It is plausible that the effects of SDB could amplify the adverse consequences of HDP given they share similar pathological pathways, and in this way SDB may also be an important contributor to fetal compromise. This study firstly aimed to confirm if the link between SDB and HDP persists after controlling for obesity, and secondly to determine whether SDB increases the risk of acute and chronic adverse maternal and fetal outcomes, both in women with HDP and normotensive controls. This study also provided the opportunity to assess, for the first time, periodic limb movements in sleep (PLMS) during pregnancy. The related disorder of restless legs syndrome (RLS) is increased two-to-three-fold in pregnant women compared to the general population, and $80 \%$ of RLS patients reportedly experience PLMS. Despite this, no studies have investigated the prevalence of PLMS during pregnancy and the possible clinical significance of this condition. This study also evaluated the presence of PLMS in late pregnancy and its relationship to HDP, subjective and objective sleep quality.
\end{abstract}

Methods: Women diagnosed with HDP and normotensive BMI-and gestation-matched controls underwent polysomnography (PSG) with time-synchronised fetal heart rate monitoring in the third trimester of pregnancy. Fetal growth was assessed by third trimester ultrasound and birthweight, customised to adjust for maternal and fetal characteristics. Maternal venous blood was sampled in the third trimester, and again at delivery in addition to fetal cord blood for markers of HDP severity, iron studies and fetal growth regulators. Medical records were reviewed for measures regarding diagnosis and severity of hypertensive disease, and for perinatal outcomes.

Results: Forty women with HDP and 40 matched controls were recruited. The frequency of SDB (respiratory disturbance index $(R D I) \geq 5$ ) in the cases was $52.5 \%$ compared to $37.5 \%$ in the controls $(p=.18)$, and the RDI overall did not differ $(p=.20)$. However, more severe SDB (RDI $\geq$ 10) was twice as common in women with HDP $(35 \%$ vs $15 \%, p=.04)$. SDB had no impact on maternal outcomes for $\mathrm{GH}, \mathrm{PE}$ or normotensive women, including gestation at diagnosis, severity of hypertension or biomarkers of disease severity. There was no temporal relationship between maternal apnoea and fetal distress on fetal heart rate monitoring, but severity of SDB was weakly related to overall fetal heart rate decelerations in controls with well-grown fetuses $(r=.44, p=$ .02). The presence of SDB had no effect on birthweight centile, third trimester fetal growth trajectory or regulators of fetal growth in cord blood. Among the HDP women, infants of those 
with SDB were actually larger at birth $(p=.02)$. PLMS were found to be very common during pregnancy, with almost half experiencing a mild degree of PLMS and a quarter more severely affected. PLMS were unrelated to hypertensive status, sleep quality or iron deficiency.

Discussion: Mild SDB occurs in half of women with HDP, but also in over a third of BMI-matched normotensive women, suggesting that the link between SDB and HDP is in part due to the confounding effect of obesity. A significant independent association between HDP and moderate SDB suggests that SDB may still play an important role in the development of hypertension during pregnancy. SDB did not affect the course of hypertensive disease in pregnancy, nor did it have a detrimental effect on our indicators of fetal health. PLMS during pregnancy is a frequently observed sleep condition of pregnancy but appears to be benign in terms of sleep quality and hypertension. This study confirms the high prevalence of mild SDB in this population. Given our findings that only more severe SDB was related to HDP, this challenges the optimal definition and threshold for clinical significance in pregnant populations. Future research needs to identify those at highest risk of pathophysiological consequences of SDB in pregnancy, delineate the proposed causative pathways and the potential of interventional clinical trials involving CPAP to improve pregnancy outcomes. 


\section{Declaration}

This thesis comprises only the authors original work towards the Doctor of Philosophy except where indicated in the preface. No other person's work has been used without due acknowledgment in the main text of the thesis. The thesis is fewer than the maximum word limit in length, exclusive of tables, bibliographies and appendices.

Danielle L. Wilson 


\section{Preface}

This thesis is submitted in the "Thesis with Publication" format approved by The University of Melbourne, where it is permissible for candidates to submit a thesis including in progress or published material as part of their thesis, along with chapters that are written specifically for the thesis.

This thesis is presented as a series of seven chapters. Chapter 1 is a comprehensive literature review that provides an introduction to the research contained in this thesis. Chapter 2 is a general method chapter outlining the study design. Chapters 3, 4, 5 and 6 each correspond to a results chapter or a manuscript published in a peer reviewed journal. Chapter 7 brings together our main results in a general discussion, sets them in context of current research, and sign posts future research directions. Preceding each empirical chapter is a short preface that outlines the rationale for each chapter and draws a link between studies in the series.

The format and style of Chapters 3 and 6 follow the specific requirements set out by the particular journal in which they were published. The exceptions to this is that, for the sake of readability, tables and figures are presented in the body of the manuscript rather than at the end, and the subheadings and in-text citation style has been aligned to the rest of the thesis. For continuity, the referencing has been incorporated into the final reference list for the entire thesis. Appendices that were not required in the manuscripts for publication have now been referred to in the text and appended at the end of the thesis. Australian English spelling has been used in the rest of the thesis chapters apart from the words fetal and fetus, as used by The Royal Australian and New Zealand College of Obstetricians and Gynaecologists (RANZCOG).

Submission to each research ethics committee, data collection, data analysis, and initial manuscript preparation were almost exclusively undertaken by the candidate Danielle Wilson. The candidate was also responsible for participant recruitment, interpretation of the results and further preparation of manuscripts. Professor Susan Walker, Associate Professor Mark Howard and Dr. Fergal O'Donoghue provided supervision, intellectual input into the overall study and revision of published manuscripts arising from this thesis. Dr. Alison Fung and Dr. Maree Barnes also provided intellectual input into the overall study and published manuscripts arising from this thesis, and Ms. Gabrielle Pell assisted with participant recruitment. Pavlina Toman assisted with sleep study analysis in order to maintain blinding to the candidate, and Dr. Hannah Skrzypek performed CTG analysis of fetal heart rate events.

All work towards this thesis was carried out following enrolment in the degree. This thesis has not been submitted for the award of any degree or diploma in any other tertiary institution. No third party editorial assistance was provided in preparation of the thesis. 
The publication status of all results chapters in this thesis is as follows -

Chapter 3 - Sleep-disordered breathing in hypertensive disorders of pregnancy - a BMImatched study - Published by Journal of Sleep Research on 25 th January 2018.

Chapter 4 - The Acute and Chronic Impact of Sleep-Disordered Breathing in Pregnancy on Maternal Outcomes - Unpublished material not submitted for publication as yet.

Chapter 5 - The Acute and Chronic Impact of Sleep-Disordered Breathing in Pregnancy on Fetal Outcomes - Unpublished material not submitted for publication as yet.

Chapter 6 - Periodic Limb Movements in Sleep During Pregnancy: A Common but Benign Disorder - Published by Sleep and Biological Rhythms on $13^{\text {th }}$ September 2017.

All research procedures in this thesis were approved by the Human Research Ethics Committees at Austin Health (Project No. H2012/04469) and the Mercy Hospital for Women (Project No. R12/02), with approvals forwarded to the Human Ethics Committee of the Faculty of Medicine, Dentistry and Health Sciences, University of Melbourne.

This research was supported by funding from the Austin Health Medical Research Foundation, Institute for Breathing and Sleep, the Australian Stillbirth Alliance and the Medical Research Foundation for Women and Babies. The candidate Danielle Wilson was supported by a National Health and Medical Research Council (NHMRC) postgraduate scholarship (APP1038747). 


\section{Publications and Presentations associated with this Thesis}

Journal publications

- Wilson DL, Walker SP, Fung AM, Pell G, O'Donoghue F, Barnes M, Howard M. Sleepdisordered breathing in hypertensive disorders of pregnancy - A BMI-matched study. $J$ Sleep Res. 2018; DOI 10.1111/jsr.12656.

- Wilson DL, Walker SP, Fung AM, O'Donoghue F, Barnes M, Howard M. Periodic limb movements in sleep during pregnancy: a common but benign disorder? Sleep Biol. Rhythms. 2017; 16(1): 11-20. DOI 10.1007/s41105-017-0125-7.

- Whitehead C, Tong S, Wilson D, Howard M, Walker SP. Treatment of early-onset preeclampsia with continuous positive airway pressure. Obstet Gynecol. 2015; 125(5): $1106-9$.

\section{Book Chapters}

- Walker SP, Wilson DL, Howard ME. Sleep in pregnancy. In: Mansfield DR, Antic N, Rajaratnam S, Naughton MT, ed. Sleep Medicine. Research, Vic: IP Communications, P/L; 2017.

Presentations at Scientific Meetings

- 2014 - Chest Annual General Meeting

Austin, Texas

Oral Presentation - CPAP Therapy in Pregnancy

- 2013 - Australasian Sleep Association 25 $5^{\text {th }}$ Annual Scientific Meeting Brisbane, Australia.

Symposium Presentation - Sleep and Breathing in the Pregnant Woman.

- 2012 - World Congress on Sleep Apnea

Rome, Italy

Oral Presentation - Maternal Sleep-Disordered Breathing and Foetal Outcomes Wilson DL, Fung A, Esdale H, Barnes M, O'Donoghue F, Howard M, Walker S.

The Australian Health \& Medical Research Congress

Adelaide, Australia.

Oral Presentation - Maternal Sleep-Disordered Breathing and Foetal Outcome. Wilson DL, Fung A, Esdale H, Barnes M, O'Donoghue F, Howard M, Walker S. 


\section{Acknowledgments}

Firstly, I would like to thank my supervisors Associate Professor Mark Howard, Professor Sue Walker and Dr Fergal O'Donoghue for their unbelievable support throughout this process. I have truly been lucky to have mentors who are so knowledgeable and esteemed within their respective fields, who have genuinely been interested in my research and have generously given their time to support me and have continued to offer their support in my future career pursuits.

I would also like to express my gratitude and give recognition to my colleagues involved in the development and undertaking of this project - Dr. Maree Barnes, Dr. Alison Fung and Ms. Gabrielle Pell. Dr. Maree Barnes has supported my research from early on in my career and although she has taken a smaller role within this project, I couldn't have gotten this far without her initial support. Dr. Alison Fung receives thanks in two ways! As well as being involved in this study, as an obstetrician she has overseen two of the most important "projects" of my life - that of my two daughters. She will always be a special person in our lives. Gabrielle Pell has been a wonderful friend and support over the past 10 years of my research career, I could not have been as successful recruiting for this project without her guidance and readiness to share her extensive knowledge as a research midwife. Gabrielle and her research team were also invaluable in collecting delivery blood samples for this project. Thanks also go to Ms. Pavlina Toman and Dr. Hannah Skrzypek for their assistance with data analyses to maintain the integrity of our findings.

I'd also like to thank all of my colleagues at the Institute for Breathing and Sleep and the Austin Sleep Laboratory. My manager at the sleep lab, Mr. Tom Churchward, has been wholly supportive of my research and combining this with my clinical work, making a PhD much easier to manage when you feel encouraged by your employer and colleagues.

In order to make this research possible, we received the support of the Mercy Hospital for Women and for that I am very grateful. Thank you to the Fetal Monitoring Unit for allowing me to linger in order to recruit the tricky-to-find patients with a hypertensive disorder, and for the birth suite and operating theatre staff who assisted in collecting those difficult blood samples at delivery.

To my family and friends, thank you for your ongoing support and understanding throughout my time as a PhD student. My husband Phil has been my rock throughout the past six years, through buying our family home and the birth of our beautiful daughter Elise, and the impending arrival of our second baby daughter. I will be forever grateful for the wonderful life we have built together and look forward to moving on from being a student and getting a 'real job' to give him a break! 


\section{Table of Contents}

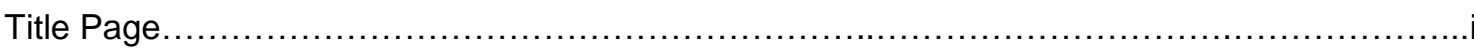

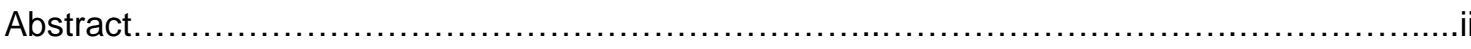

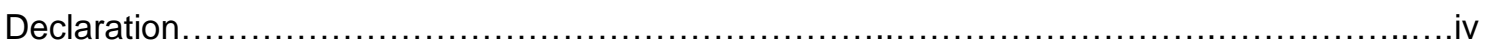

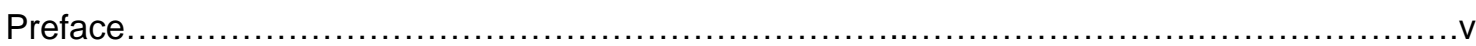

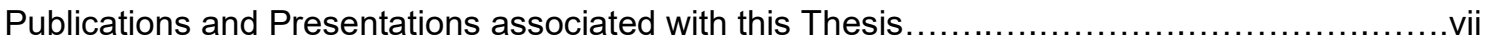

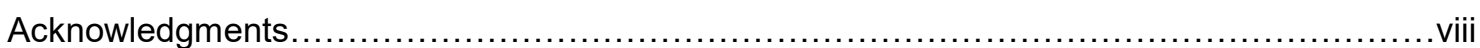

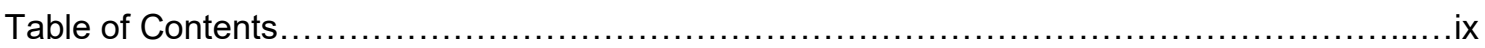

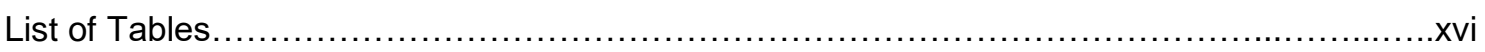

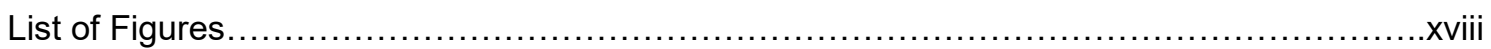

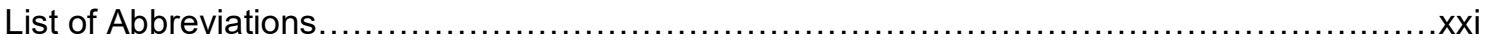

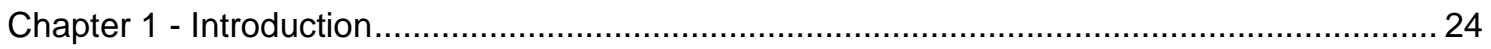

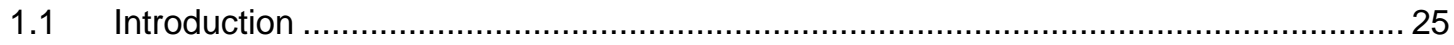

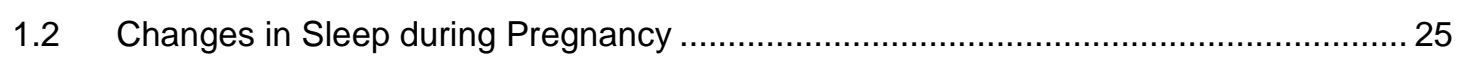

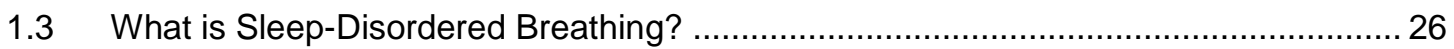

1.3.1 Assessment of Sleep-Disordered Breathing - Polysomnography ....................... 27

1.3.2 Epidemiology and Risk Factors for Sleep-Disordered Breathing ......................... 29

1.3.3 Effects of Sleep-Disordered Breathing on Health ....................................... 30

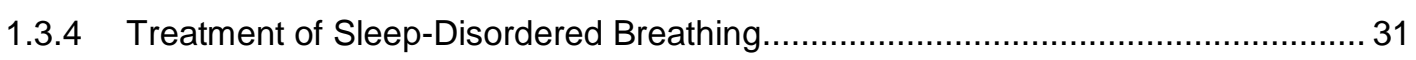

1.4 Sleep-Disordered Breathing in Pregnancy ..................................................... 32

1.4.1 Prevalence of Sleep-Disordered Breathing in Pregnancy.............................. 33

1.4.1.1 Self-Reported Snoring and Sleep-Disordered Breathing Symptoms ............. 33

1.4.1.2 Objective Data for Sleep-Disordered Breathing Prevalence in Pregnancy..... 33

1.4.2 Relevance of Sleep-Disordered Breathing in Pregnancy.................................... 35

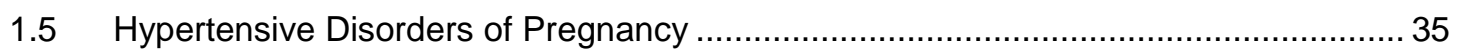

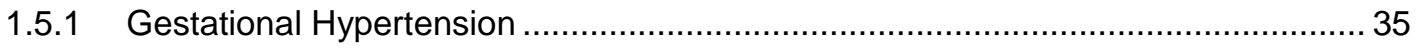

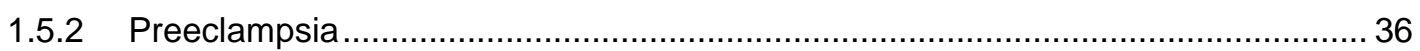

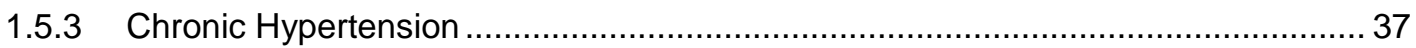

1.5.4 Preeclampsia Superimposed on Chronic Hypertension................................... 38

1.5.5 Maternal and Fetal Outcomes associated with Hypertensive Disorders of

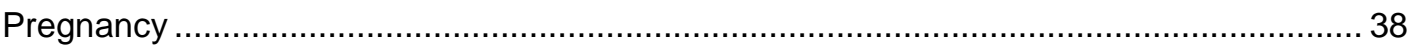

1.6 Proposed Relationship between Sleep-Disordered Breathing and Hypertensive

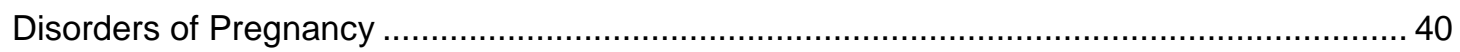

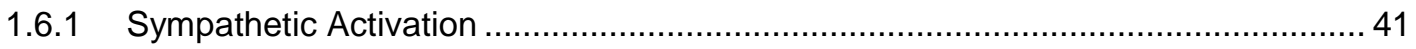

1.6.2 Oxidative Stress, Inflammation and Endothelial Dysfunction ............................ 42

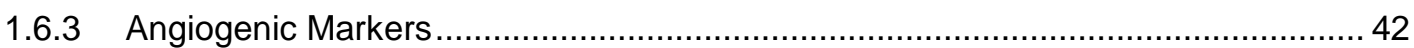

1.7 Sleep-Disordered Breathing and Fetal Health ............................................... 43 


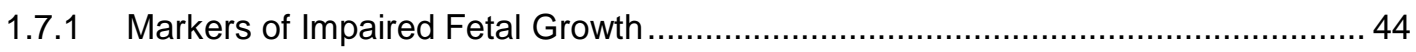

1.7.1.1 Measurement of Impaired Fetal Growth - Ultrasound ................................... 45

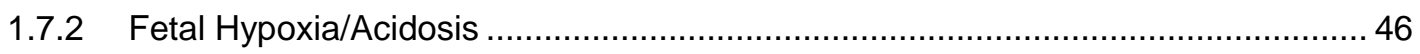

1.7.2.1 Measurement of Fetal Hypoxia/Acidosis - Cardiotocography …..................... 46

1.8 Sleep-Disordered Breathing and Hypertensive Disorders of Pregnancy - Current

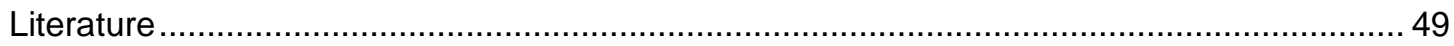

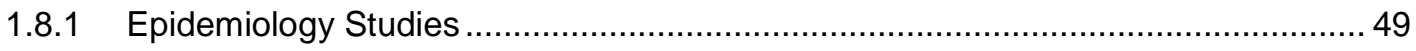

1.8.2 Potential Mechanisms of Sleep-Disordered Breathing and Hypertensive

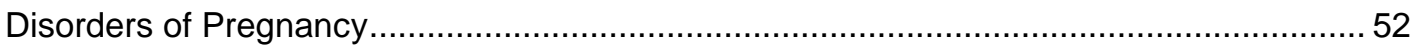

1.9 The Impact of Sleep-Disordered Breathing on other Maternal Outcomes .................... 53

$1.10 \quad$ The Impact of Sleep-Disordered Breathing on Fetal Outcomes ............................ 54

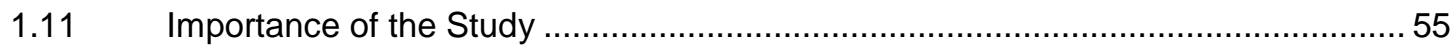

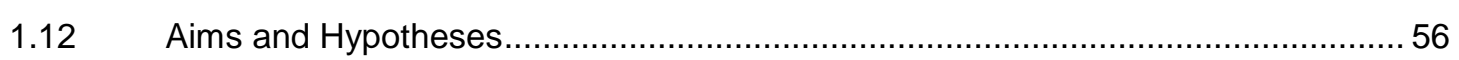

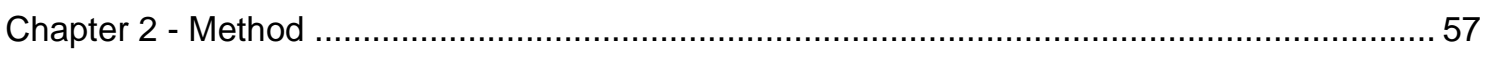

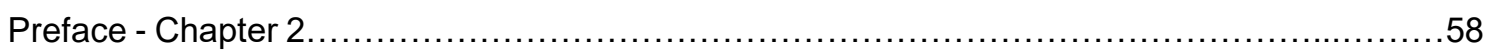

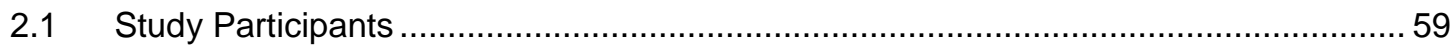

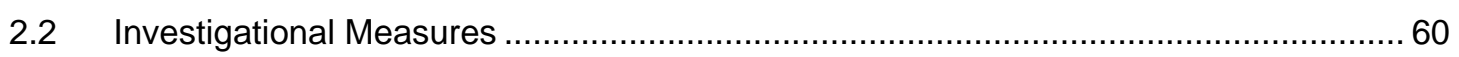

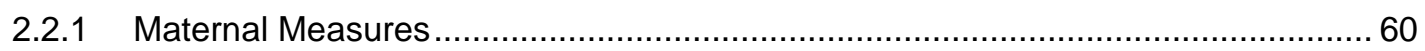

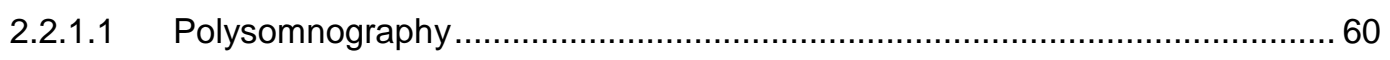

2.2.1.2 Overnight Blood Pressure Measurement ...................................................... 61

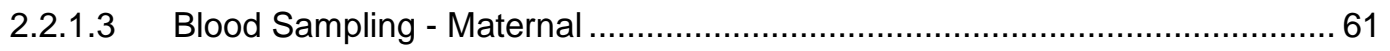

2.2.1.4 Prediction of Sleep-Disordered Breathing Questionnaires................................ 61

2.2.1.5 Medical Records - Maternal/Obstetric Outcomes ........................................... 62

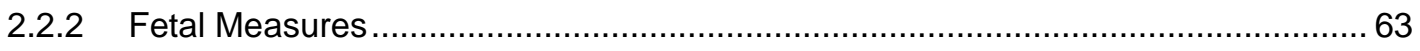

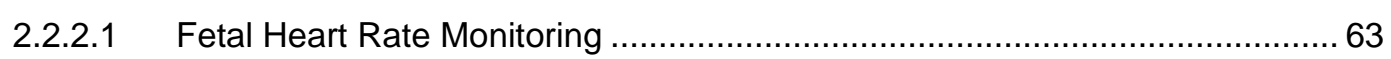

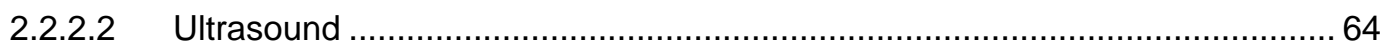

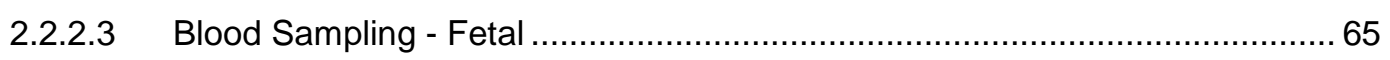

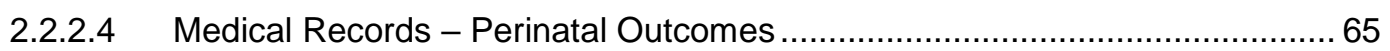

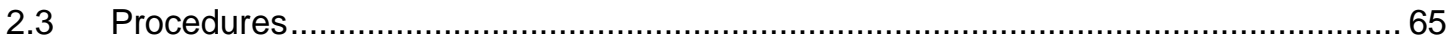

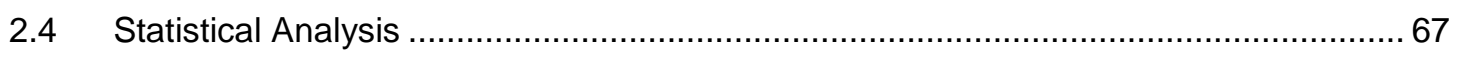

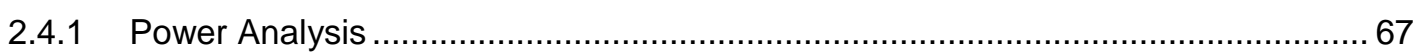

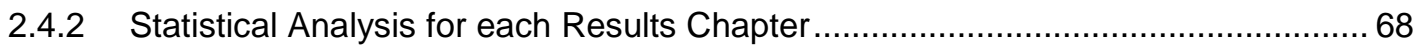

Chapter 3 - Sleep-Disordered Breathing in Hypertensive Disorders of Pregnancy -

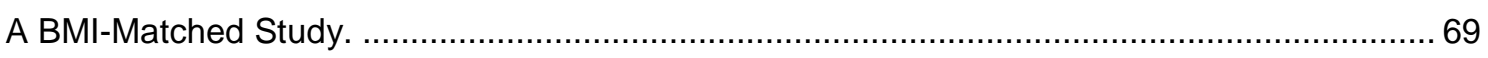

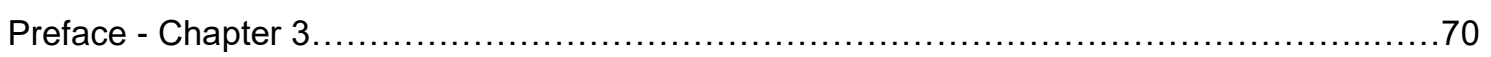

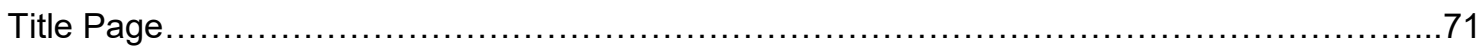

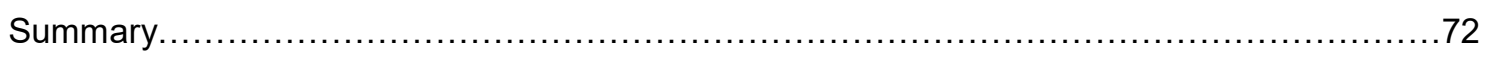




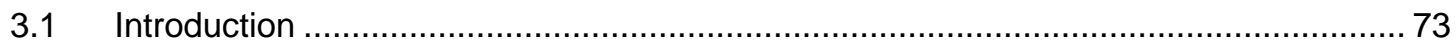

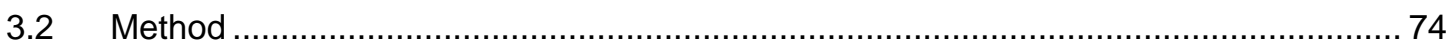

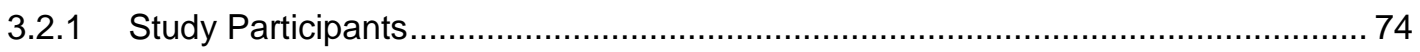

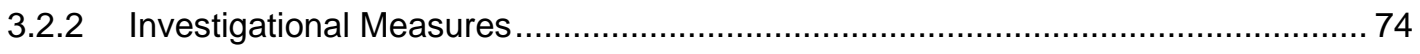

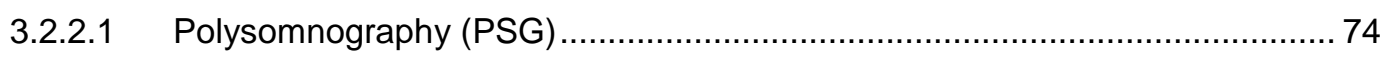

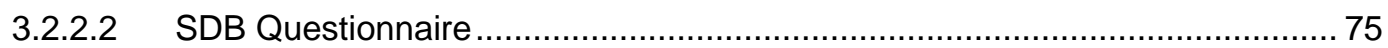

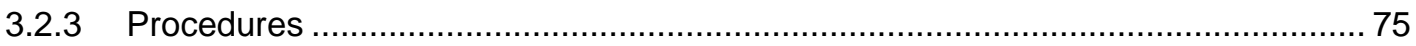

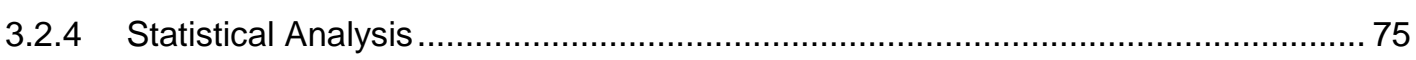

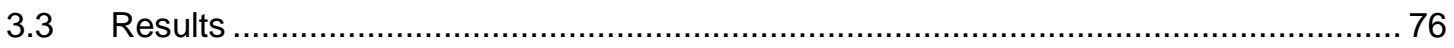

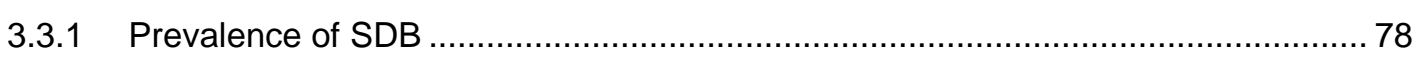

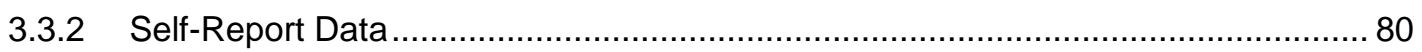

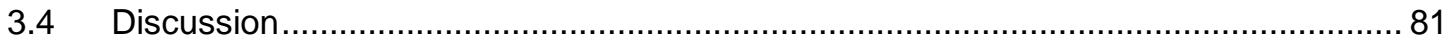

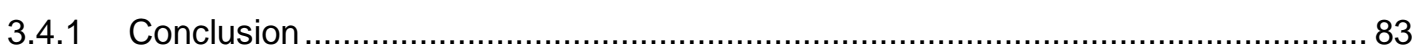

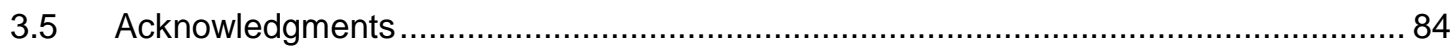

Chapter 4 - The Acute and Chronic Impact of Sleep-Disordered Breathing in Pregnancy on

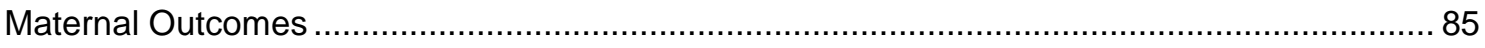

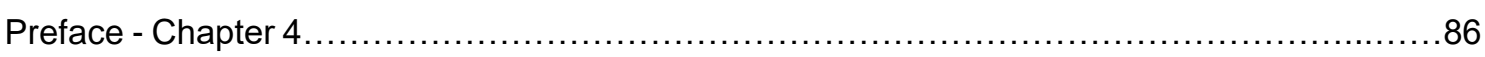

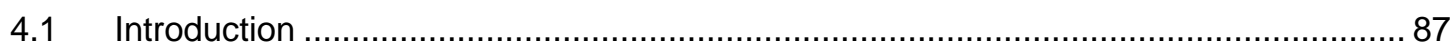

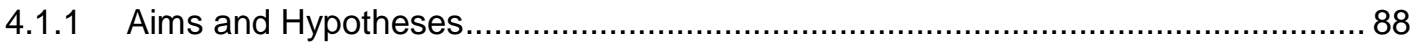

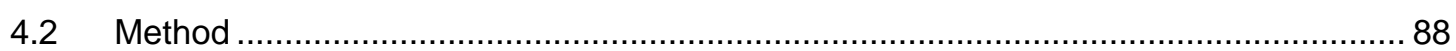

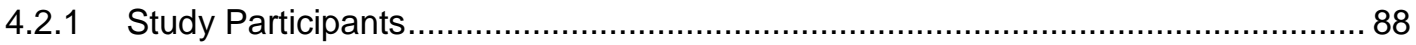

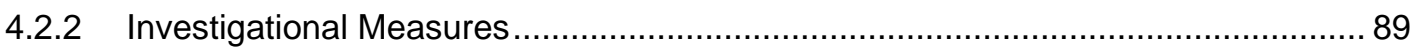

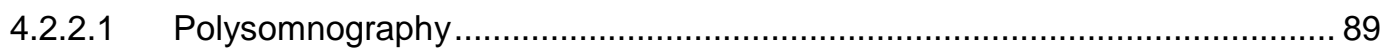

4.2.2.2 Overnight Blood Pressure Measurement …............................................... 89

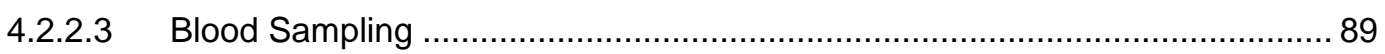

4.2.2.4 Medical Records for Maternal Outcomes ...................................................... 89

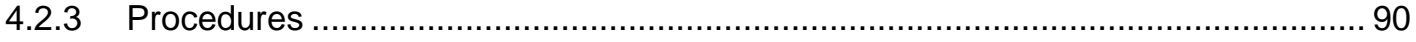

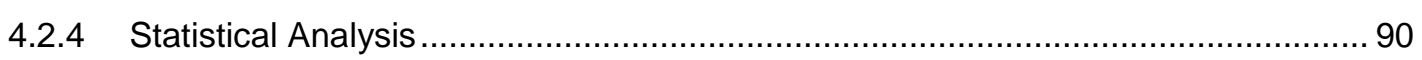

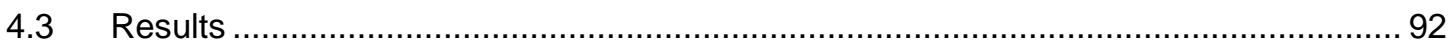

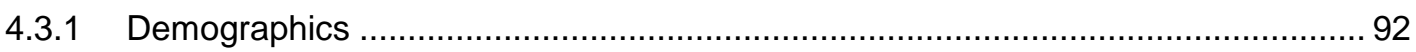

4.3.2 Hypertensive Disease and Blood Pressure Control .............................................. 94

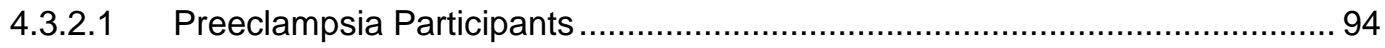

4.3.2.1.1 Gestational Age at Diagnosis of Preeclampsia ....................................... 94

4.3.2.1.2 Severity of Preeclampsia ................................................................... 94

4.3.2.1.3 Blood Pressure across Pregnancy - Preeclampsia ................................ 95

4.3.2.1.4 Biochemical and Haematological Indicators of Disease Severity................98

4.3.2.1.5 Overnight Blood Pressure on Sleep Study - Preeclampsia ....................... 98

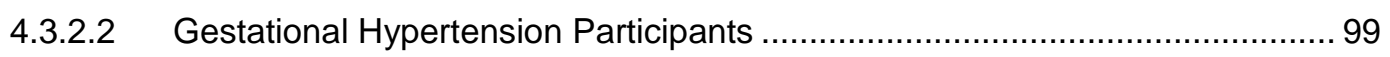

4.3.2.2.1 Gestation at Diagnosis of Gestational Hypertension ................................ 99 
4.3.2.2.2 Severity of Gestational Hypertension

4.3.2.2.3 Blood Pressure across Pregnancy - Gestational Hypertension................ 101

4.3.2.2.4 Biochemical and Haematological Indicators of Disease Severity ............ 102

4.3.2.2.5 Overnight Blood Pressure on Sleep Study - Gestational Hypertension .. 102

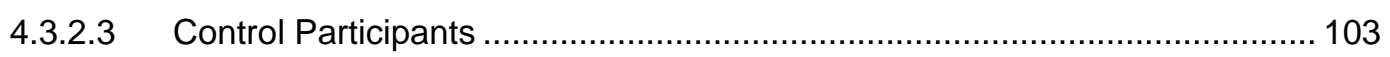

4.3.2.3.1 Diagnosis of Hypertensive Disorders of Pregnancy .............................. 103

4.3.2.3.2 Severity of Hypertensive Disease ....................................................... 103

4.3.2.3.3 Blood Pressure across Pregnancy - Controls........................................... 103

4.3.2.3.4 Overnight Blood Pressure on Sleep Study - Controls ........................... 104

4.3.3 Angiogenic Markers of Hypertensive Disease .................................................... 105

4.3.4 Severity of Sleep-Disordered Breathing and its Impact on Hypertensive

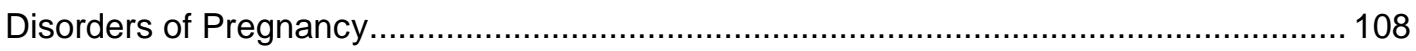

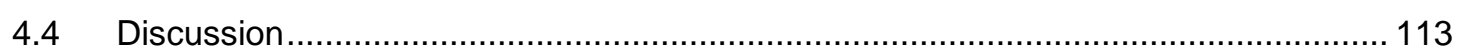

4.4.1 Diagnosis and Severity of Hypertensive Disease …........................................ 113

4.4.1.1 Preeclampsia Development and the Potential Impact of Sleep-Disordered

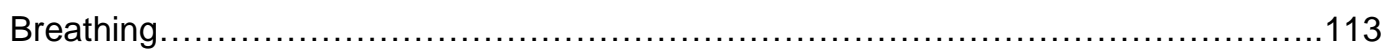

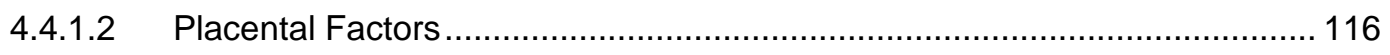

4.4.1.2.1 Anti-Angiogenic Factors - Soluble Flt-1 and Soluble Endoglin ............... 116

4.4.1.2.2 Pro-Angiogenic Factor - Placental Growth Factor ............................... 116

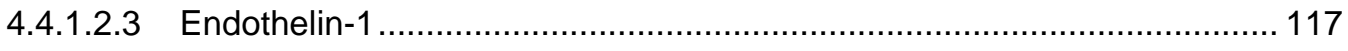

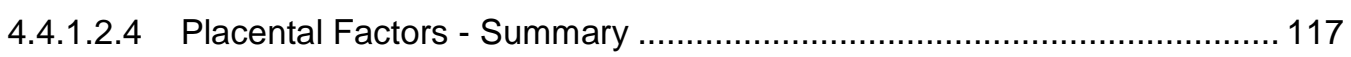

4.4.2 Blood Pressure Control during Pregnancy ......................................................... 118

4.4.2.1 Hypertension in Pregnancy and the Impact of Sleep-Disordered

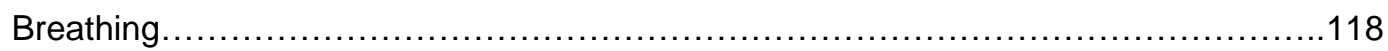

4.4.2.2 Nocturnal Blood Pressure and Sleep-Disordered Breathing ........................ 120

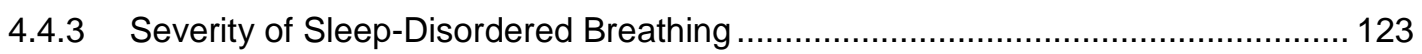

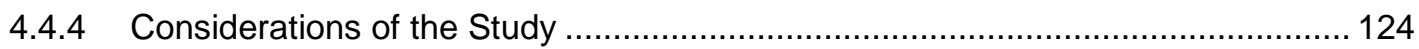

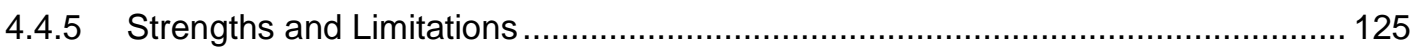

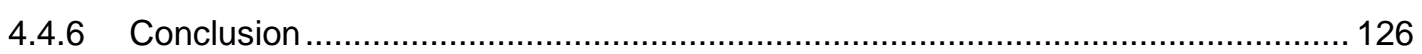

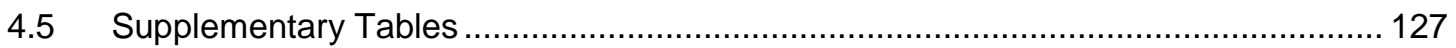

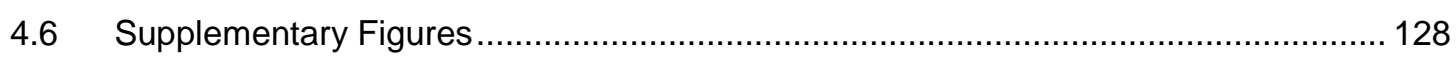

Chapter 5 - The Acute and Chronic Impact of Sleep-Disordered Breathing in Pregnancy

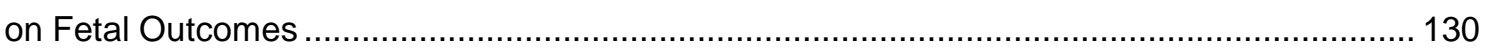

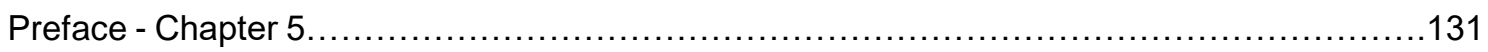

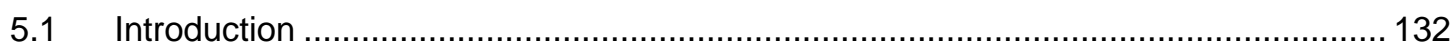

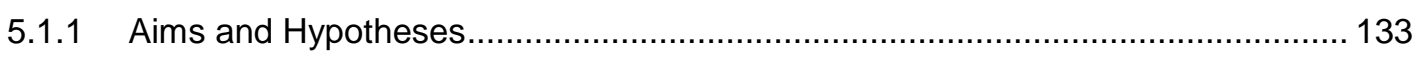

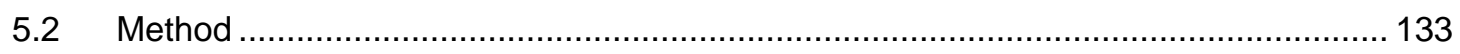

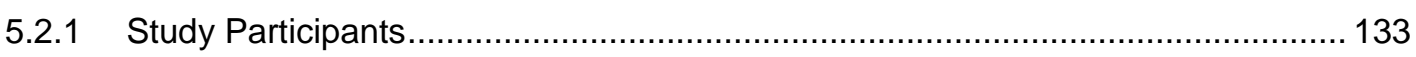

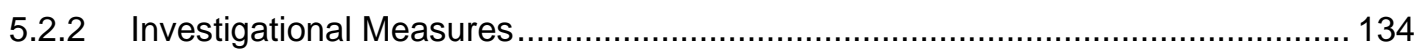




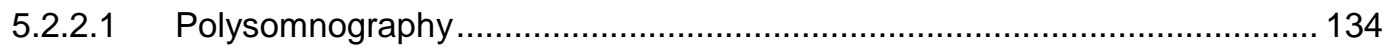

5.2.2.2 Fetal Heart Rate Monitoring …........................................................... 134

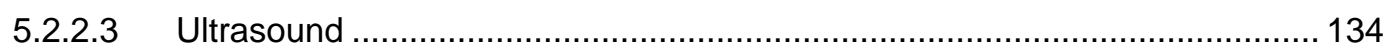

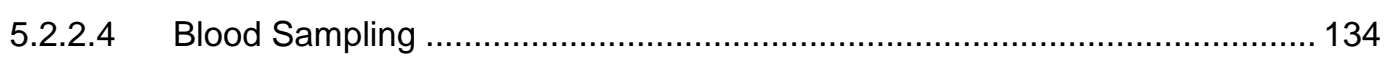

5.2.2.5 Medical Records for Fetal Outcomes ...................................................... 135

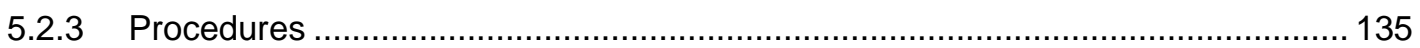

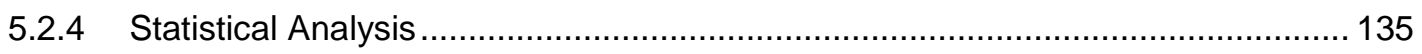

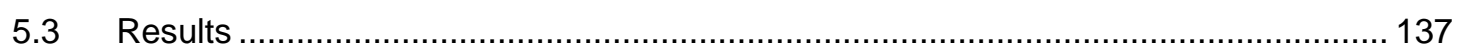

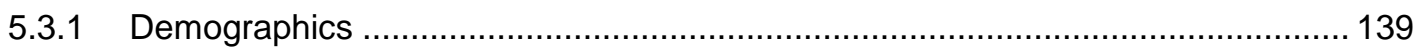

5.3.2 The Impact of Sleep-Disordered Breathing on Short-Term Indices of Fetal

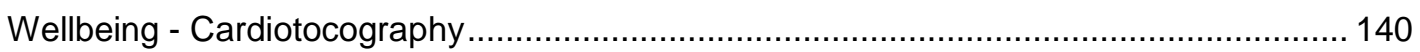

5.3.2.1 Overall Summary of Cardiotocography Results .......................................... 140

5.3.2.2 Indicators of Fetal Distress on Cardiotocography ....................................... 141

5.3.2.2.1 Impact of Sleep-Disordered Breathing Overall ........................................ 141

5.3.2.2.2 Impact of Sleep-Disordered Breathing in Hypertensive Disorders of

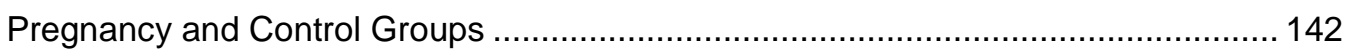

5.3.2.2.3 Multivariate Analysis of Risk Factors for Fetal Distress on

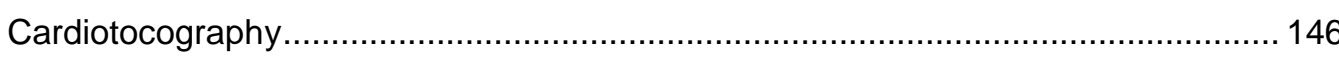

5.3.2.2.4 Severity of Sleep-Disordered Breathing and Fetal Distress on

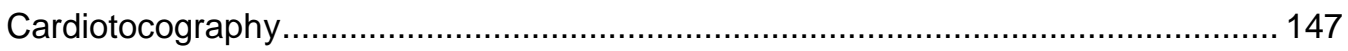

5.3.2.2.5 Relationship between Fetal Growth Restriction and Cardiotocography ... 148

5.3.3 The Impact of Sleep-Disordered Breathing on Long-Term Indices of Fetal

Wellbeing - Fetal Growth and Perinatal Outcomes........................................................ 148

5.3.3.1 Fetal Growth and Sleep-Disordered Breathing ............................................ 148

5.3.3.2 Fetal Growth and Perinatal Outcomes - Impact of Sleep-Disordered

Breathing in Hypertensive Disorders of Pregnancy and Control Groups ....................... 150

5.3.3.2.1 Hypertensive Disorders of Pregnancy Group ...................................... 150

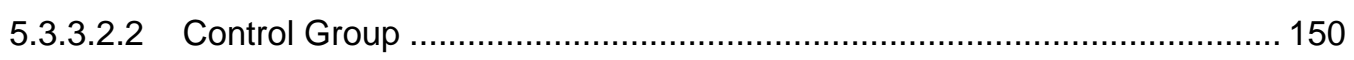

5.3.3.2.3 Impact of Hypertensive Disease on Fetal Growth and Perinatal

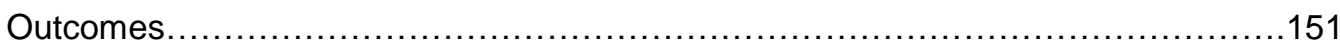

5.3.4 The Impact of Sleep-Disordered Breathing on Long-Term Indices of Fetal Wellbeing - Biomarkers of Fetal Growth in Cord Blood .................................................. 153

5.3.4.1 Biomarkers of Fetal Growth - Impact of Sleep-Disordered Breathing in Hypertensive Disorders of Pregnancy and Control Groups ........................................ 153

5.3.4.2 Biomarkers of Fetal Growth - Impact of Hypertensive Disease.................... 154

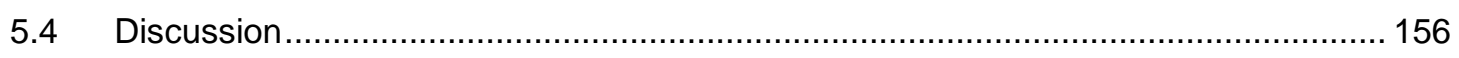

5.4.1 Acute Impact of SDB on Fetal Wellbeing - Cardiotocography............................ 156

5.4.2 Chronic Impact of Sleep-Disordered Breathing on Fetal Wellbeing ..................... 159

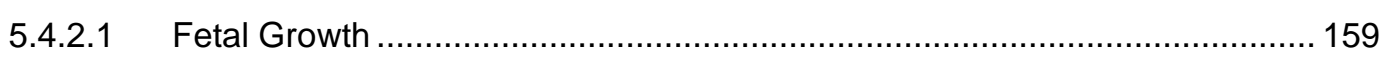

5.4.2.1.1 Fetal Growth and Hypertensive Disease .......................................... 159 
5.4.2.1.2 Fetal Growth and Sleep-Disordered Breathing in Hypertensive and

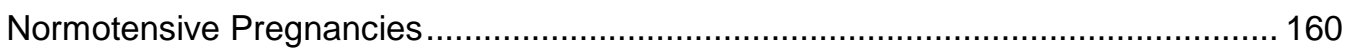

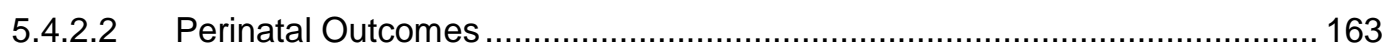

5.4.2.3 Fetal Growth Hormones in Cord Blood .................................................. 164

5.4.2.3.1 Fetal Growth Hormones in Cord Blood and Sleep-Disordered

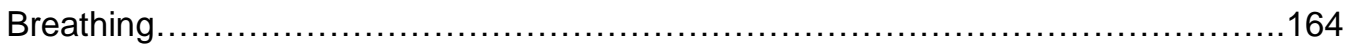

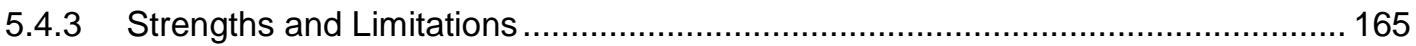

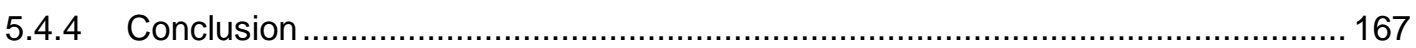

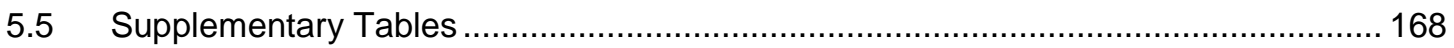

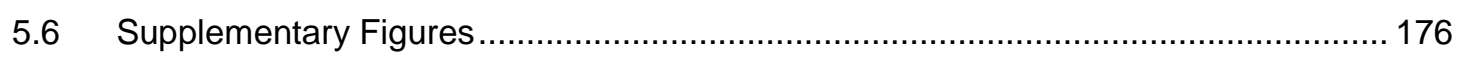

Chapter 6 - Periodic Limb Movements in Sleep During Pregnancy: A Common but Benign

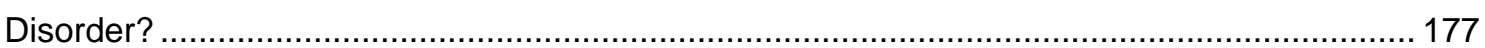

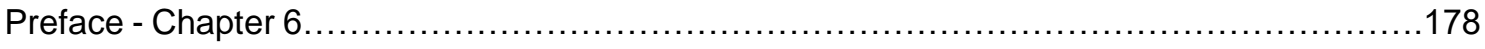

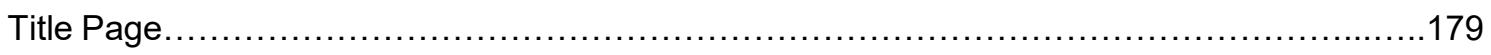

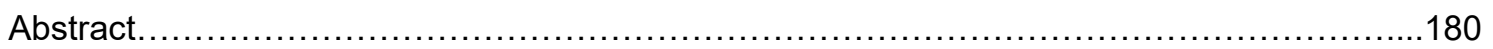

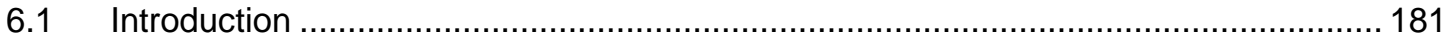

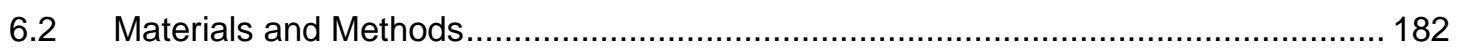

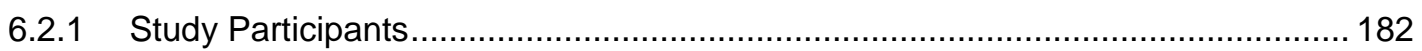

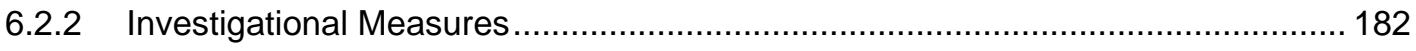

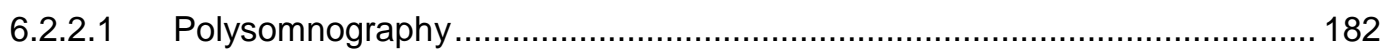

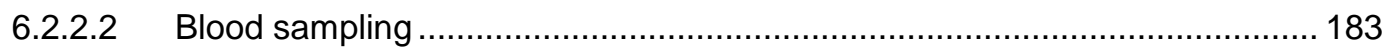

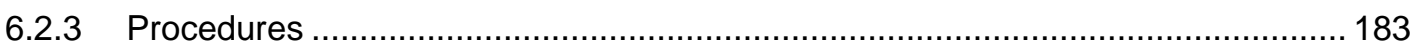

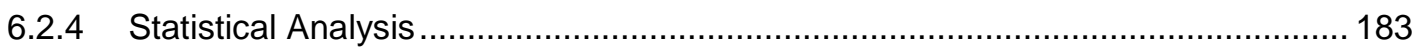

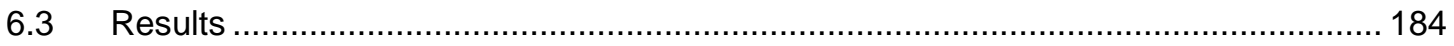

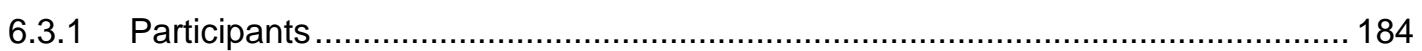

6.3.2 Periodic Limb Movements during Sleep............................................................ 184

6.3.3 Restless Legs Syndrome Questionnaire …................................................... 187

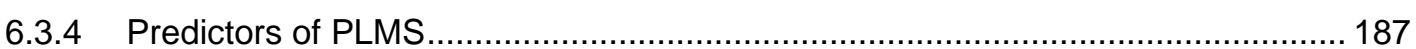

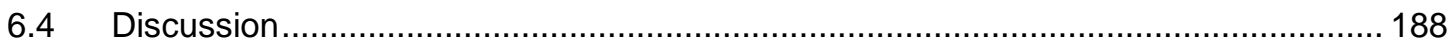

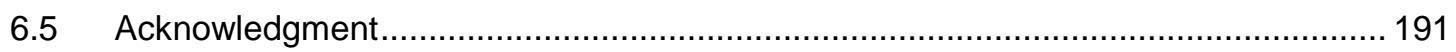

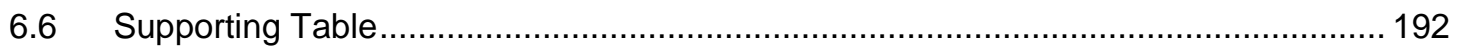

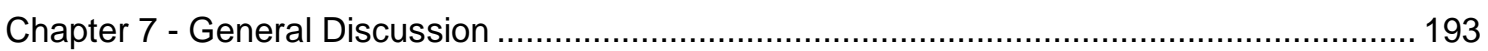

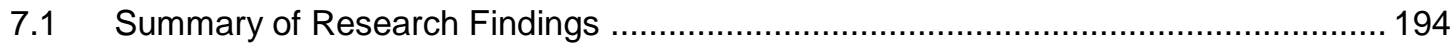

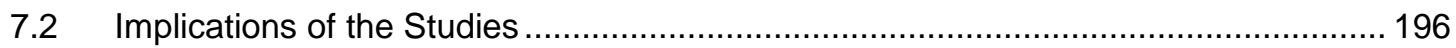

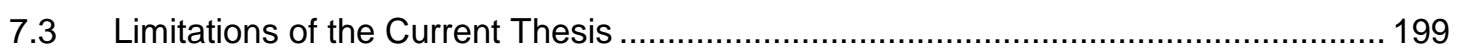

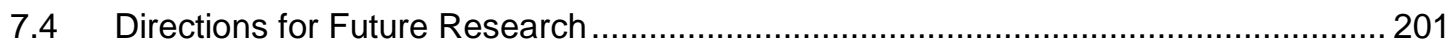

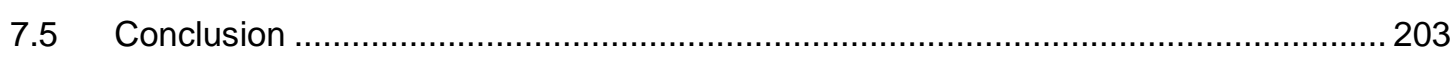


Reference List.

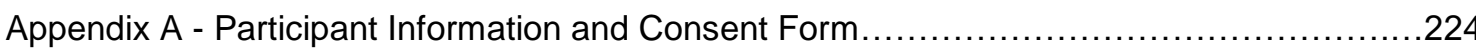

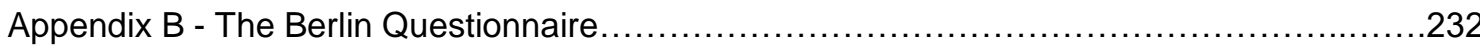

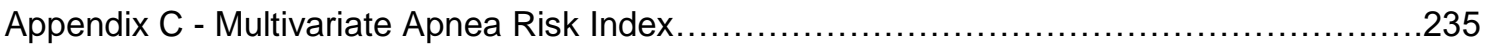

Appendix D - Restless Legs Syndrome Questionnaire .........................................238 


\section{List of Tables}

Chapter 2

Table 2.1 The Revised ISSHP Definition of Preeclampsia (2014) 59

Chapter 3

Table 3.1 Demographics for Gestational Hypertension and Preeclampsia Cases with Matched Controls

Table 3.2 RDI Categories for Gestational Hypertension and Preeclampsia Cases with

Matched Controls

Table 3.3 Indices of SDB for Gestational Hypertension and Preeclampsia Cases with

Matched Controls

Table 3.4 Sleep Quality for Gestational Hypertension and Preeclampsia Cases with

Matched Controls. 80

Table 3.5 Berlin Questionnaire Results.... 81

Chapter 4

Table 4.1 Demographics for Preeclampsia and Gestational Hypertension Cases and Normotensive Control Groups Stratified by SDB Status

Table 4.2 Booking and Peak Blood Pressure for Preeclampsia and Gestational

Hypertension Cases and Normotensive Control Groups Stratified by SDB

Status

Table 4.3 Biochemical and Haematological Measures of Preeclampsia Severity for the

Preeclampsia and Gestational Hypertension Group Stratified by SDB Status .98

Table 4.4 Change Per Week for Endothelin-1, Soluble Flt-1, Soluble Endoglin and

Placental Growth Factor 106

Table 4.5 Key Results for Each Group with SDB Defined as an RDI $\geq 15$ 109

Table 4.6 Key Blood Pressure Results for Each Group with SDB Defined as an RDI $\geq 15 \ldots . . .110$

Table 4.7 SDB defined as RDI $\geq 15$ - Change Per Week for Endothelin-1, Soluble Flt-1,

Soluble Endoglin and Placental Growth Factor 112

Table 4.8 Third Trimester (A), Delivery (B) and Change Per Week Values for

Endothelin-1, Soluble Flt-1, Soluble Endoglin and Placental Growth Factor for the Preeclampsia versus Gestational Hypertension Groups, and the

Gestational Hypertension/Preeclampsia versus Control Groups 127

Chapter 5

Table 5.1 Demographics for Hypertensive Disorders of Pregnancy Cases and Normotensive Controls Stratified by SDB Status. 140

Table 5.2 Cardiotocography Variables for SDB vs No SDB Groups. 141 
Table 5.3 Cardiotocography Variables for Hypertensive Disorders of Pregnancy Cases and Normotensive Controls stratified by SDB

Table 5.4 Ordinal Regression - Univariate Relationships Between Demographic Variables and Number of Fetal Heart Rate Events per Hour on Cardiotocography

Table 5.5 Factors associated with Number of Fetal Heart Rate Events per hour on Cardiotocography on Stepwise Ordinal Regression Modelling 147

Table 5.6 Relationship between Fetal Heart Rate Events on Cardiotocography and Fetal Growth 148

Table 5.7 Measures of Fetal Growth in the SDB vs No SDB groups 149

Table 5.8 Measures of Fetal Growth and Perinatal Outcomes for those with and without SDB in the Hypertensive Disorders of Pregnancy and Control Groups 152

Table 5.9 Demographics for Preeclampsia and Gestational Hypertension Cases Stratified by SDB Status. 168

Table 5.10 Cardiotocography Variables across Hypertensive Groups 169

Table 5.11 Key Fetal Outcome Measures for those with and without Maternal SDB at an $\mathrm{RDI} \geq 15$ 170

Table 5.12 Measures of Fetal Growth for those with and without SDB across

Preeclampsia and Gestational Hypertension Groups

Table 5.13 Measures of Fetal Growth across Hypertensive Groups ..................................... 172

Table 5.14 Birth Outcomes across Hypertensive Groups ................................................... 173

Table 5.15 Birth Outcomes for those with and without SDB across Hypertensive Groups ...... 173

Table 5.16 Biomarkers of Fetal Growth in Cord Blood - Hypertensive Groups by SDB

Status 174

Table 5.17 Biomarkers of Fetal Growth in Cord Blood across Hypertensive Groups 175

Chapter 6

Table 6.1 PLMS Indices for Normotensive and Hypertensive Participants. 185

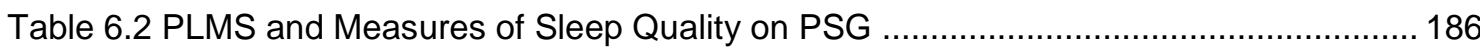

Table 6.3 PLMS and Iron Studies. 186

Table 6.4 Factors associated with PLMSI > 15/hr during Pregnancy on Stepwise Logistic Regression Model 187

Table 6.5 Demographic and RLS Symptoms associated with PLMSI > 15/hr during Pregnancy 192 


\section{List of Figures}

Chapter 1

Figure $1.1 \mathrm{a}$ ) Polysomnogram (PSG) showing normal breathing during stage N3 sleep 28

Figure $1.1 \mathrm{~b}$ ) Polysomnogram (portable) showing obstructive sleep apnoea with associated oxygen desaturations during REM sleep. .28

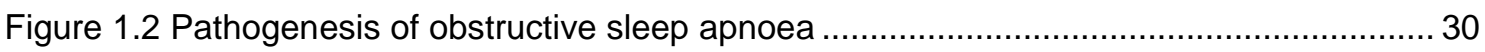

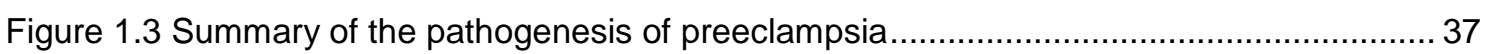

Figure 1.4 Potential interaction between SDB and hypertensive disorders of pregnancy ......... 40

Figure 1.5 Recordings of sympathetic nerve activity (SNA), respiration (RESP) and blood pressure (BP) during 3 min of stage II sleep .................................................. 41

Figure 1.6 Potential interaction between SDB and acute and chronic fetal compromise ........... 44

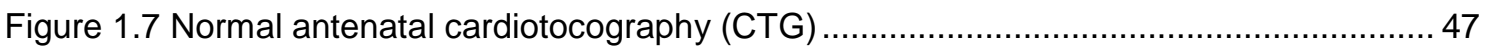

Figure 1.8 Example of prolonged fetal bradycardia on antenatal cardiotocography (CTG) at 32 weeks gestation

\section{Chapter 2}

Figure 2.1 Illustration of electrode placement on the maternal abdomen for the Monica AN24 fetal heart rate monitor (Monica Healthcare website)

Figure 2.2 Diagram highlighting key procedures involved in the study at planned gestational age of pregnancy

Chapter 3

Figure 3.1 Consort diagram.

Figure 3.2 Percentage of participants in each Respiratory Disturbance Index (RDI/hr) category for the GH and PE groups.

Figure 3.3 Respiratory Disturbance Index (RDI/hr) across different sleep stages and positions for the $\mathrm{GH} / \mathrm{PE}$ group compared to the control group

Chapter 4

Figure 4.1 Survival curve for the number of days from diagnosis of preeclampsia (PE) until delivery, with the covariate of fetal growth restriction (FGR), for the SDB and No SDB groups.

Figure 4.2 Mean \pm SEM systolic and diastolic blood pressure across pregnancy for the SDB group and the No SDB group within the preeclampsia group

Figure 4.3 Mean \pm SEM overnight systolic and diastolic blood pressure on the night of the sleep study for the PE group, for those with and without SDB.

Figure 4.4 Survival curve for the number of days between diagnosis of gestational hypertension $(\mathrm{GH})$ and delivery with gestation of diagnosis of $\mathrm{GH}$ as a covariate, for the SDB and No SDB groups. 
Figure 4.5 Mean \pm SEM systolic and diastolic blood pressure (BP) across pregnancy for the SDB group and the No SDB group within the gestational hypertension (GH) group ..... 101

Figure 4.6 Mean \pm SEM overnight blood pressure on the night of the sleep study for the gestational hypertension (GH) group, for those with and without SDB 102

Figure 4.7 Mean \pm SEM systolic and diastolic blood pressure (BP) across pregnancy for the SDB group and the No SDB group within the control group. 104

Figure 4.8 Mean \pm SEM overnight blood pressure on the night of the sleep study for the control group, for those with and without SDB

Figure 4.9 Median values (with IQR) for endothelin-1 (ET-1), soluble fms-like tyrosine kinase-1 (sFlt-1), soluble endoglin (sEng) and placental growth factor (PIGF) measured in the third trimester and at delivery......

Figure 4.10 Median values (with IQR) for endothelin-1 (ET-1), soluble fms-like tyrosine kinase-1 (sFlt-1), soluble endoglin (sEng) and placental growth factor (PIGF) measured in the third trimester and at delivery, with SDB defined as RDI $\geq 15 \ldots . .111$

Figure 4.11 Polysomnogram (PSG) showing BP response to obstructive respiratory events with oxygen desaturations to a nadir of $77 \%$, during REM sleep in one of our participants with $\mathrm{GH}$ at $36+5$ weeks of gestation.

Figure 4.12 Endothelin-1 (ET-1), soluble fms-like tyrosine kinase-1 (sFlt-1), soluble endoglin (sEng) and placental growth factor (PIGF) measured in the third trimester and at delivery for each participant in the GH/PE group - stratified by SDB $(\mathrm{RDI} \geq 5)$

Figure 4.13 Endothelin-1 (ET-1), soluble fms-like tyrosine kinase-1 (sFlt-1), soluble endoglin (sEng) and placental growth factor (PIGF) measured in the third trimester and at delivery for each participant in the control group - stratified by SDB $(R D I \geq 5)$

Chapter 5

Figure 5.1 Potential interaction between SDB and acute and chronic fetal compromise 132

Figure 5.2 Flow chart of participant data available for each fetal outcome. 138

Figure 5.3 Time-synchronised cardiotocography (CTG) with polysomnography (PSG) across a 10 minute period of NREM sleep

Figure 5.4 Time-synchronised cardiotocography (CTG) with polysomnography (PSG) across a 10 minute period of REM sleep

Figure 5.5 Growth trajectory between estimated fetal weight (EFW) from ultrasonography in the third trimester to birthweight using customised centiles 153

Figure 5.6 Insulin-like Growth Factor 1 and 2 (IGF-1, IGF-2) and Binding Protein 1 and 2 (IGFBP-1, IGFBP-2) for the SDB vs. No SDB group within the HDP and control group 
Figure 5.7 Scatterplot demonstrating relationship between RDI (log transformed) and total number of fetal heart rate (FHR) events on cardiotocography (CTG) per hour. 176

Chapter 6

Figure 6.1 Systolic (Sys) and diastolic (Dias) blood pressure $(\mathrm{M} \pm \mathrm{SD}$ ) measured prior to bedtime and upon morning awakening..... 185 


\section{List of Abbreviations}

AASM = American Academy of Sleep Medicine

AFP $=$ alpha feto-protein

$\mathrm{AHI}=$ apnoea-hypopnoea index

$\mathrm{ALT}=$ alanine transaminase

ANOVA $=$ analysis of variance

aOR $=$ adjusted odds ratio

AT1-AA = Angiotension II Type I Receptor Activating Autoantibodies

$\mathrm{BMI}=$ body mass index

$\mathrm{BP}=$ blood pressure

CEFM = continuous electronic fetal monitoring

$\mathrm{Cl}=$ confidence interval

$\mathrm{CNS}=$ central nervous system

$\mathrm{CPAP}=$ continuous positive airway pressure

$\mathrm{CRP}=\mathrm{C}$-reactive protein

$\mathrm{CTG}=$ cardiotocography

$\mathrm{DIC}=$ disseminated intravascular coagulation

$E C G$ = electrocardiogram

EDS = excessive daytime sleepiness

$E E G$ = electroencephalogram

EFW = estimated fetal weight

$E H G$ = electro hysterogram

$E M G$ = electromyogram

$E O G=$ electrooculogram

EPO = erythropoietin

ESS $=$ Epworth Sleepiness Scale

$\mathrm{ET}-1$ = endothelin-1

FGR = fetal growth restriction

$\mathrm{FHR}=$ fetal heart rate

GDM = gestational diabetes mellitus

$\mathrm{GH}=$ gestational hypertension

HDP = hypertensive disorders of pregnancy

HTN = hypertension

$\mathrm{ICU}=$ intensive care unit

IGF = insulin-like growth factor

IGFBP = insulin-like growth factor binding protein

IL-6 = interleukin-6

IRLSSG = International Restless Legs Syndrome Study Group

ISSHP = International Society for the Study of Hypertension in Pregnancy 
IUGR = intrauterine growth restriction

MAP = multivariate apnea risk

MAPI = multivariate apnea risk index

$\mathrm{MHR}=$ maternal heart rate

$\mathrm{N} 1$ = stage 1 sleep

$\mathrm{N} 2$ = stage 2 sleep

N3 = stage 3 sleep

$\mathrm{NICU}=$ neonatal intensive care unit

$\mathrm{NK}=$ natural killer

$\mathrm{NPV}=$ negative predictive value

nRBCs $=$ nucleated red blood cells

NREM = non-rapid eye movement

$\mathrm{ODI}=$ oxygen desaturation index

$\mathrm{OR}=$ odds ratio

OSA = obstructive sleep apnoea

OSAS = obstructive sleep apnoea syndrome

$\mathrm{PAP}=$ positive airway pressure

$\mathrm{PE}=$ preeclampsia

PIGF = placental growth factor

PLM = periodic limb movement

PLMD = periodic limb movement disorder

PLMS = periodic limb movements in sleep

PLMs $=$ periodic limb movements

PLMSI = periodic limb movements in sleep index

$\mathrm{PO}_{2}=$ partial pressure of oxygen

$\mathrm{PPV}=$ positive predictive value

$\operatorname{Pr}: \mathrm{cr}=$ protein:creatinine

$P S G=$ polysomnography

$\mathrm{RBC}=$ red blood cell

$\mathrm{RDI}=$ respiratory disturbance index

REM = rapid eye movement

RERA = respiratory event related arousal

$\mathrm{RLS}=$ restless legs syndrome

$\mathrm{SCN}=$ special care nursery

$\mathrm{SDB}=$ sleep-disordered breathing

sEng $=$ soluble endoglin

sFlt-1 = soluble fms-like tyrosine kinase-1

$\mathrm{SpO}_{2}=$ peripheral capillary oxygen saturation

SWS = slow wave sleep

T3 $=$ third trimester 
TGF- $\beta 1=$ transforming growth factor- $\beta 1$

TNF- $\alpha=$ tumor necrosis factor alpha

TST $=$ total sleep time

UARS = upper airway resistance syndrome

$\mathrm{uE3}=$ estriol

VEGF = vascular endothelial growth factor 
Chapter 1 - Introduction

Sleep-Disordered Breathing in Hypertensive Disorders of Pregnancy:

Impact on Maternal and Fetal Outcomes 


\section{$1.1 \quad$ Introduction}

Preeclampsia (PE) is a serious and progressive multi-system disorder which affects up to $8 \%$ of pregnancies. PE and its associated complications account for around $15 \%$ of direct maternal mortality and $10 \%$ of perinatal mortality, and worldwide it is responsible for the deaths of tens of thousands of mothers and babies each year. ${ }^{1-3}$ There are limited treatment options with delivery of the fetus and the pathological placenta the only definitive management. Gestational hypertension (GH), defined as the new onset of hypertension after 20 weeks of gestation, is the main component of PE and up to 1 in 4 women with $\mathrm{GH}$ go on to develop this disorder.

In the non-pregnant population, sleep-disordered breathing (SDB) is associated with hypertension and cardiovascular disease, ${ }^{4,5}$ potentially as a result of episodes of hypoxia contributing to hypertension through sympathetic activation and oxidative stress, and the activation of inflammatory pathways leading to endothelial dysfunction. Pregnant women are at an increased risk of SDB,, 7 and recent data has shown an association between SDB and adverse pregnancy outcomes including hypertensive disorders of pregnancy (HDP) ${ }^{8,9}$ It is plausible that the effects of SDB may amplify the already negative consequences of $\mathrm{GH}$ and $\mathrm{PE}$ through similar pathological pathways. This study aims to employ objective measurement techniques to confirm the relationship between SDB and HDP, and to determine whether SDB increases the risk of other adverse maternal and fetal outcomes.

\subsection{Changes in Sleep during Pregnancy}

Sleep complaints are commonly reported during pregnancy, primarily due to the many anatomical and physiological changes that occur. A number of questionnaire and interview-based studies have documented the frequency of sleep problems during pregnancy, with symptoms typically increasing across trimesters. ${ }^{10-13}$ By the third trimester, over $95 \%$ of pregnant women reported multiple wake periods overnight or poor overall sleep quality. ${ }^{10,11}$ Hutchison et al. ${ }^{14}$ found that only $29 \%$ of third trimester pregnant women rated their sleep quality as very good or fairly good, compared with $82 \%$ rating their sleep this way before pregnancy. Primary sleep problems noted during pregnancy were frequent awakenings, difficulty falling asleep and staying asleep, needing to change sleep position and daytime sleepiness. ${ }^{10,14}$ Common reasons for waking included nocturia, fetal movements, positional discomfort, back pain and leg cramps. ${ }^{10,13,14}$

Sleep difficulties are generally attributed to physical discomfort, however these changes may be related to the development of a sleep disorder. For example, clinically significant insomnia was reported by $50.5 \%$ of pregnant women in a cohort of 689 women ${ }^{11}$ and by $54.3 \%$ of third trimester women in a study of almost 200 women. ${ }^{12}$ Excessive daytime sleepiness (EDS) has consistently been reported by approximately one-third of pregnant women. ${ }^{11,12,14}$ Comparatively, a sample of over 34,000 adults in the United States found a prevalence of self-reported insomnia and EDS for those under 45 years of age of $15.8 \%$ and $12.7 \%$ respectively, with prevalence being higher amongst women compared to 
men. ${ }^{15}$ Increased insomnia and sleepiness in pregnancy is important to consider given the implications for mood, daytime functioning and increased risk for adverse events such as motor vehicle accidents.

Another common sleep-related disorder, Restless Legs Syndrome (RLS), has been extensively studied during pregnancy with prevalence rates reported as between $10 \%$ and $34 \% .{ }^{16-22} \mathrm{RLS}$ is a neurologic condition characterised by an urge to move, that occur or worsen at rest and is relieved by activity. ${ }^{23}$ One of the central characteristics of RLS is the worsening of symptoms in the evening and during the night, with a majority of patients reporting difficulty falling asleep or waking shortly after sleep onset. Symptoms usually develop or increase with advancing pregnancy and often resolve quickly postpartum. ${ }^{16}$ RLS during pregnancy is frequently misdiagnosed or unrecognised by clinicians ${ }^{21,24}$ even though it adversely affects maternal sleep initiation and duration, sleep quality, and daytime functioning. ${ }^{16,24,25}$ Severe RLS is also associated with increased risk of cardiovascular disease. ${ }^{26,27}$ The majority of people with RLS have stereotyped repetitive movements during sleep, a distinct condition known as periodic limb movements in sleep (PLMS). Although RLS has been extensively investigated, there is no specific research evaluating PLMS and pregnancy and therefore the prevalence of PLMS during pregnancy is undetermined. ${ }^{28}$

The cause of PLMS remains unknown, and the clinical significant has been a subject of debate. Each stereotyped limb movement (typically lower limb) is accompanied by a rapid rise in heart rate and arterial blood pressure (BP), followed by a return to baseline values. Hundreds of these movements each night could hypothetically contribute to increased risk of hypertensive disease, due to the repetitive autonomic surges during sleep. ${ }^{29}$ Only two studies have reported on PLMS during pregnancy. All 10 mothers in a study on multiple gestation pregnancy exhibited PLMS, ${ }^{30}$ and Dzaja et al. ${ }^{31}$ showed that 10 pregnant women diagnosed with RLS showed more periodic leg movements (PLMs) on overnight PSG before and after delivery, compared to those without RLS.

SDB, the most common form of which is obstructive sleep apnoea (OSA), is becoming more recognised as a potential comorbidity of pregnancy. Mounting evidence suggests that SDB may develop or worsen during pregnancy and affect maternal and fetal health. This serious disorder of sleep will become the focus of this review.

\subsection{What is Sleep-Disordered Breathing?}

SDB is a descriptive term for the spectrum of disorders characterised by increased upper airway resistance during sleep. The mildest form of SDB is snoring, and the most severe form is OSA.

Snoring is a sound produced by the vibrating structures of the upper airway. The International Classification of Sleep Disorders defines snoring as a "respiratory sound generated in the upper airway during sleep that typically occurs during inspiration but may also occur in expiration". ${ }^{2}$ Its main clinical significance is that it is a marker of obstructive sleep apnoea syndrome (OSAS) and upper airway resistance syndrome (UARS), and is the most common reason for referral to sleep laboratories. ${ }^{33}$ 
OSA occurs when the soft tissues of the upper airway collapse repeatedly during sleep, causing upper airway obstruction and cessation of airflow, often resulting in falls in blood oxygen saturation. OSAS is the most common organic sleep disorder causing excessive daytime somnolence. ${ }^{34}$

Clinically, OSAS presents with a characteristic history of habitual snoring, witnessed apnoeas overnight and EDS, as recurrent episodes of hypoxia result in arousal from sleep, and culminate in sleep fragmentation. Acute hemodynamic changes occur in patients with OSA. These patients experience repetitive nocturnal oscillations in heart rate and $\mathrm{BP}$, with sudden increases in heart rate and arterial BP occurring immediately following apnoea termination. These changes are likely attributable primarily to the effects of oxygen desaturation and reoxygenation, and arousal. ${ }^{35}$

\subsubsection{Assessment of Sleep-Disordered Breathing - Polysomnography}

Overnight polysomnography (PSG), or a 'sleep study', is the gold standard method used to confirm a diagnosis of SDB. A single-night PSG is usually sufficient to diagnose or to exclude SDB. ${ }^{36}$ A sleep study confirms the presence of apnoeas (cessation of airflow $\geq 10$ seconds), and hypopnoeas (discernible reduction in airflow $\geq 10 \mathrm{~s}$ associated with either oxyhaemoglobin desaturation or a cortical arousal, detected by electroencephalography), and is able to quantify the frequency of these events. Severity of SDB is classified using the apnoea-hypopnoea index ( $\mathrm{AHI}$ ), which is the number of apnoeas or hypopnoeas recorded per hour of sleep. There is no general agreement about assigning severity descriptors to indices of SDB, however most commonly mild SDB is defined as an AHI of 5-15, moderate disease an $\mathrm{AHI}$ of 15-30 and severe disease an $\mathrm{AHI}$ of greater than $30 .{ }^{37}$ The scoring guidelines introduced in $2007^{38}$ also specify rules for optional scoring of respiratory event related arousals (RERAs), defined by a sequence of breaths $\geq 10$ seconds characterised by increasing respiratory effort (measured by oesophageal pressure) or flattening of the nasal airflow leading to an arousal from sleep, in the absence of apnoeas or hypopnoeas. With the inclusion of RERAs, a summary measure called the respiratory disturbance index $(\mathrm{RDI})$ is calculated, which represents the sum total of apnoeas, hypopnoeas, and RERAs per hour of sleep. Other commonly used results, such as the extent and frequency of oxygen desaturations (oxygen desaturation index; ODI), can also be tallied and reported. ${ }^{36}$ A full PSG will also record signals for electrocardiogram (ECG), thoracic and abdominal respiratory effort (used to categorise apnoea and hypopnoea episodes into obstructive, central or mixed based on the presence or absence of respiratory effort during the entirety or some part of the breathing event), ${ }^{37}$ leg movements, snoring and body position.

PSG also allows objective measurement of sleep stages using electroencephalography (EEG) together with electromyogram (EMG) and electrooculogram (EOG) so that sleep can be classified into different sleep stages according to a set of criteria. ${ }^{38}$ Rapid eye movement (REM) sleep is characterised by the presence of rapid eye movements, and a global abolition of muscle tone. Non-rapid eye movement (NREM) sleep is divided into several stages, corresponding to increasing sleep depth. Stages N1 and N2 correspond to light sleep, with Stage N2 characterised by K complexes and sleep spindles. Stage N3 (also known as slow wave sleep (SWS)) has an increase in slow oscillations as sleep deepens, and 
muscle tone decreases compared to the lighter sleep stages. Throughout the night, the NREM and REM sleep periods alternate following an ultradian cycle, with SWS invariably preceding REM sleep in healthy individuals. ${ }^{39}$ An example of normal breathing during sleep and OSA is shown in Figures 1.1a and $1.1 \mathrm{~b}$.

a)

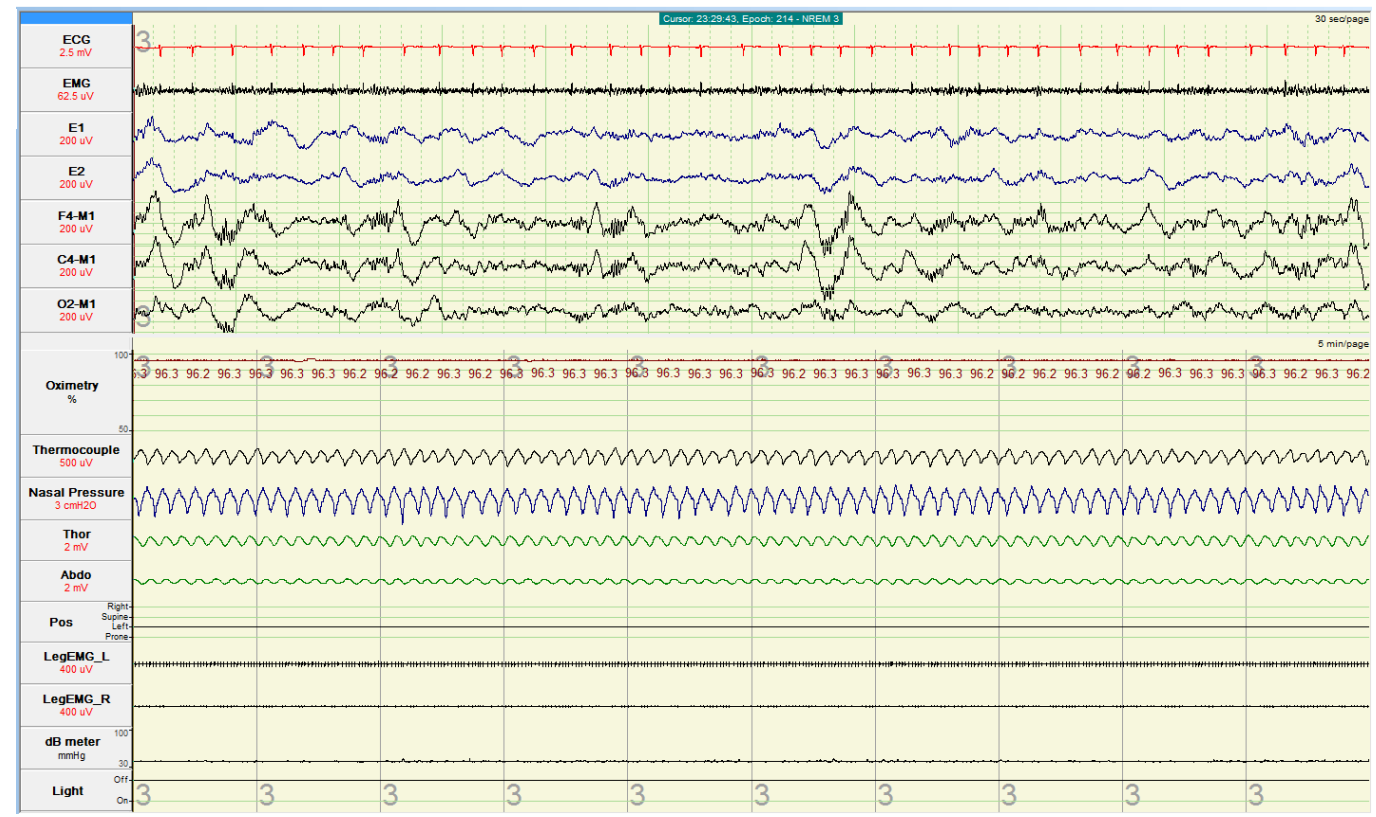

b)

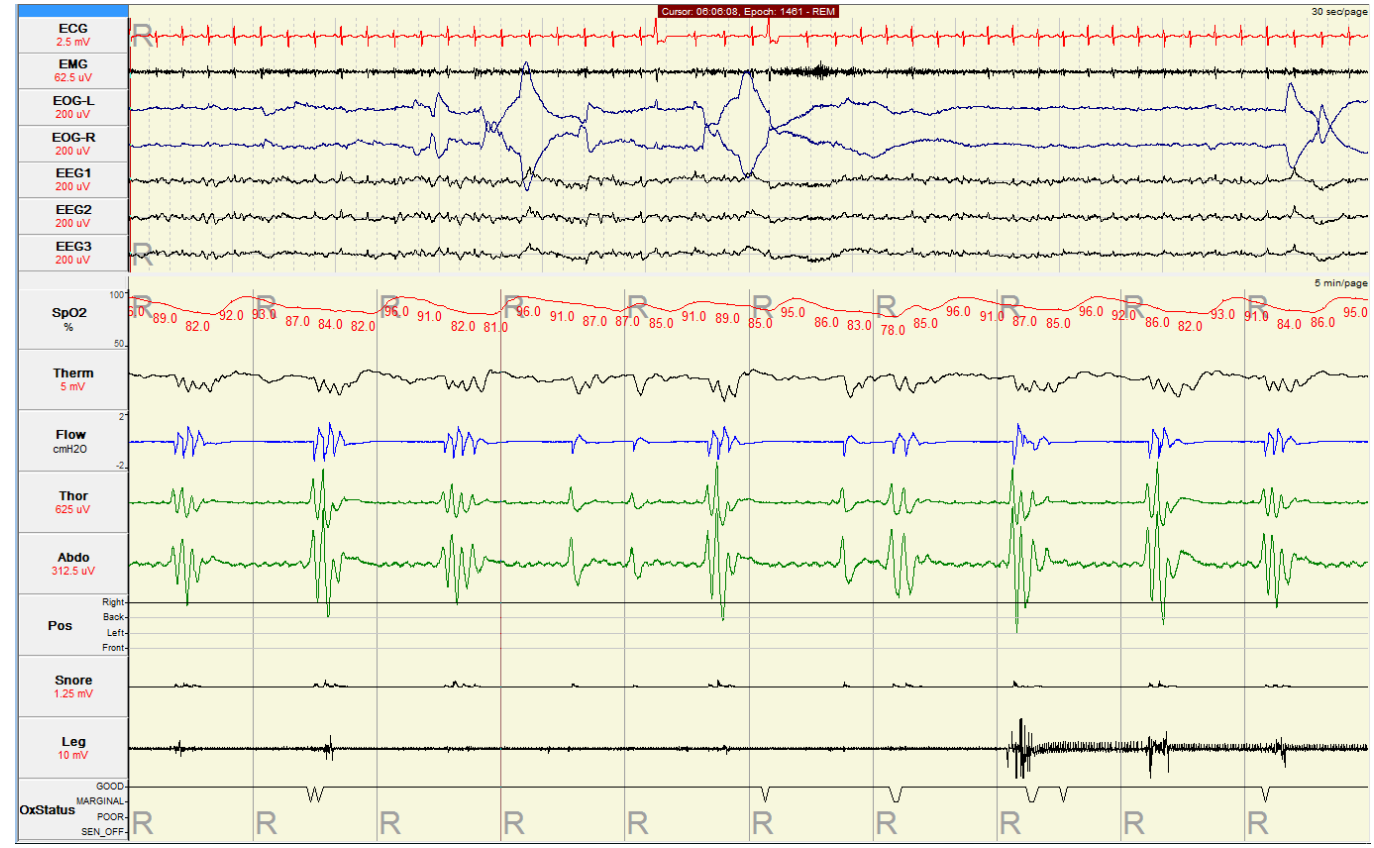

Figure 1.1 a) Polysomnogram (PSG) showing normal breathing during stage N3 sleep. The top panel shows signals involved in determining sleep stage, in a $30 \mathrm{sec}$ epoch. The bottom panel shows signals mostly related to respiration, in a $5 \mathrm{~min}$ epoch. The following signals are recorded for a full PSG - ECG = electrocardiogram, EMG = electromyogram, E1 = left electrooculogram, $E 2$ = right electrooculogram, F4-M1, C4-M1 and O2-M1 = EEG derivations referenced to $M 1$, oximetry = arterial oxygen saturation, thermocouple, nasal 
pressure, Thor $=$ thoracic respiratory effort, $A b d o=$ abdominal respiratory effort, Pos $=$ body position, LegEMG_L = left leg movements, LegEMG_R = right leg movements, $d B$ meter = measurement of sound i.e. snoring, and Light = light sensor.

b) Polysomnogram (portable) showing obstructive sleep apnoea with associated oxygen desaturations during REM sleep. The cessation of airflow for $>10 \mathrm{sec}$ with continued (but reduced) respiratory effort indicates cyclical obstructive apnoeas. Though containing the same signals, the different labels for an in-home study include EOG-L = left electrooculogram, EOG- $R=$ right electrooculogram, EEG1 $=$ F4-M1, EEG2 $=$ C4-M1, EEG3 = O2-M1, SpO2 = arterial oxygen saturation, Therm = thermocouple, Flow = nasal pressure, Thor $=$ thoracic respiratory effort, $A$ Abdo $=$ abdominal respiratory effort, $P o s=$ body position, Snore = snoring, Leg = left and right leg movements combined and OxStatus = oximetry status.

\subsubsection{Epidemiology and Risk Factors for Sleep-Disordered Breathing}

The first large epidemiologic PSG study of SDB estimated that $2 \%$ of women and $4 \%$ of men in the middle-aged workforce met the minimal diagnostic criteria for the OSAS, defined as an AHI of 5 and daytime hypersomnolence. Up to $9 \%$ of women and $24 \%$ of men in this study had an AHI of 5 without daytime somnolence. ${ }^{40}$ In the study by Bixler and co-workers, ${ }^{41}$ the prevalence of clinically defined sleep apnoea among premenopausal women was $0.6 \%$, with the prevalence increasing after menopause.

SDB is associated with multiple anatomic risk factors. Many patients with SDB are obese and have a large neck or crowded upper airway. In those with a normal body habitus, common structural abnormalities such as narrow nasal passage, rhinitis, long soft palate, large tonsils, or retroflexed mandible leading to a small airway, contribute to airway obstruction. ${ }^{42}$

Severity of SDB can be influenced by factors such as sleep stage and body position. REM sleep in patients with SDB is typically associated with increased frequency of obstructive events that are often prolonged and accompanied with severe oxygen desaturation..$^{43}$ REM-related SDB, in which respiratory events occur predominantly in REM sleep, accounts for a significant proportion of the general SDB population. ${ }^{44-46}$ Worsening of SDB severity during REM sleep is likely related to the accompanying atonia resulting in more prominent airway collapse. ${ }^{45}$ Reviews have shown that supine sleep is consistently associated with more severe SDB indices in adults. ${ }^{47,48}$ More than half of SDB patients are considered to be "positional", in that they have at least twice as many apnoeas and/or hypopnoeas during sleep in the supine compared to the lateral position. ${ }^{45,48}$ The basis for supine-related worsening of SDB severity likely relates to gravity-driven collapse of the tongue and pharyngeal soft tissue to occlude the airway. ${ }^{48}$ 


\subsubsection{Effects of Sleep-Disordered Breathing on Health}

OSA is associated with a number of biochemical and cellular abnormalities, and the metabolic and endocrine sequelae of OSA may contribute to increased cardiovascular morbidity as summarised in Figure 1.2.49 The cycle of apnoea and recovery causes hypoxaemia/reoxygenation and hypercapnia/hypocapnia; these periods result in sympathetic activation with elevated catecholamines. ${ }^{50,51}$ Baroreceptor sensitivity may also be altered, resulting in sustained hypertension. ${ }^{52}$ Recurrent episodes of hypoxia and reoxygenation activate neutrophils, which adhere to endothelium and generate reactive oxygen species. Endothelial dysfunction results ${ }^{53}$ and contributes to the development of atherosclerosis. OSA is associated with elevations in proinflammatory cytokines interleukin-6 (IL-6), tumor necrosis factor alpha (TNF- $\alpha$ ) and C-reactive protein (CRP), ${ }^{54-57}$ which may contribute to atherosclerosis, endothelial dysfunction, insulin resistance and type 2 diabetes. Leptin levels are positively correlated with $\mathrm{AHI}, 58$ and have been implicated as an independent marker of cardiovascular risk. ${ }^{59}$ Adiponectin is an adipokine with insulin-sensitizing, anti-atherogenic and antiinflammatory properties. It is reduced by proinflammatory cytokines and the levels of this hormone are lower in patients with OSA.60,61

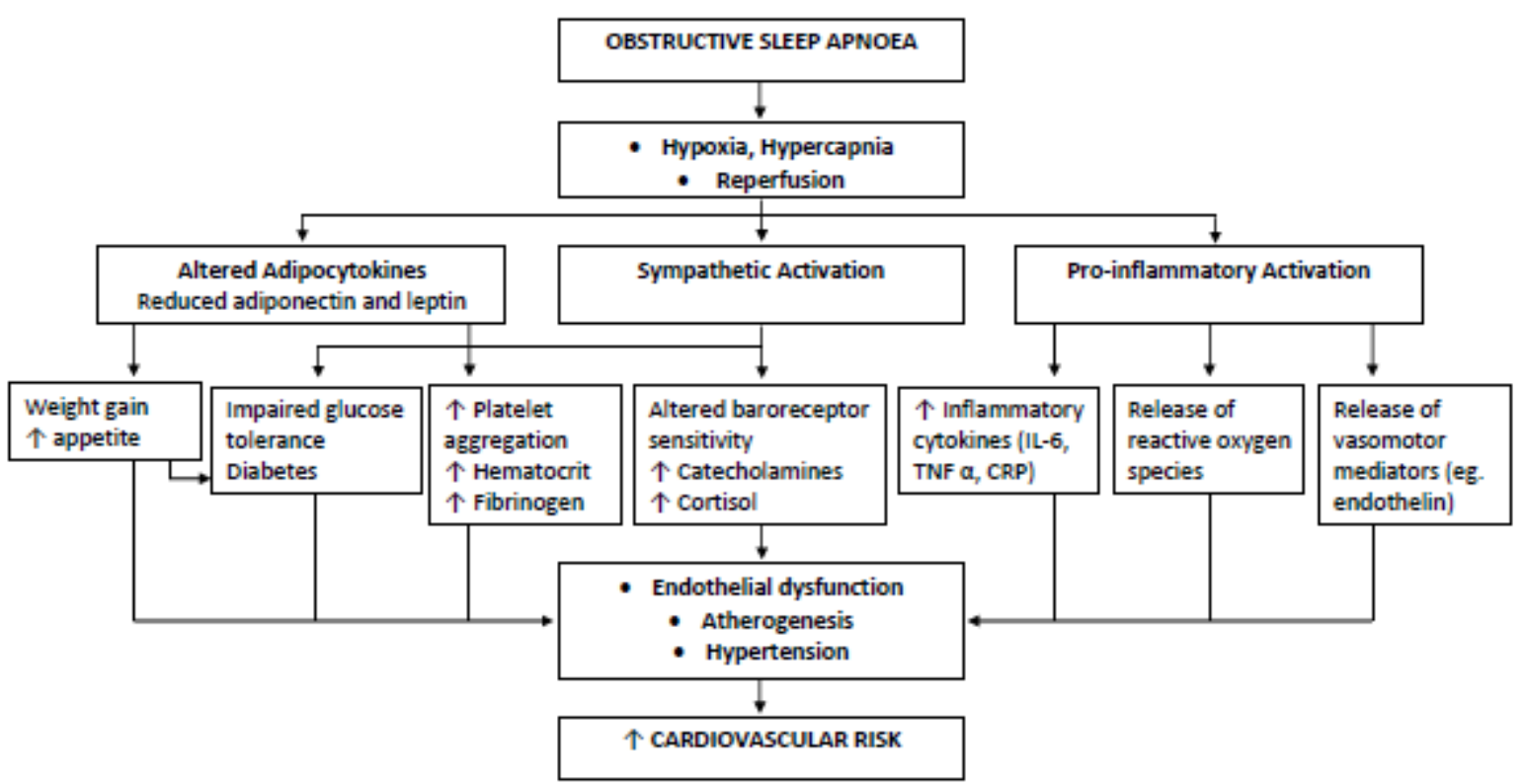

Figure 1.2 Pathogenesis of obstructive sleep apnoea. IL-6 = interleukin-6; TNF- $\alpha=$ tumor necrosis factor- $\alpha 49$

In the general population, SDB is associated with significant health risks and lowered quality of life measures. Longitudinal studies have demonstrated that moderate to severe sleep apnoea is independently associated with a large increased risk of all-cause mortality. ${ }^{62-64}$ In particular, SDB has been consistently linked to hypertension and cardiovascular disease. Individuals with PSG-indicated 
SDB have 2 to 3 times greater odds of having or developing hypertension than those without SDB, after adjustment for potential confounding factors. ${ }^{4}$ The incidence of new hypertension has also been shown to be significantly dependent on baseline level of OSA, strongly suggesting a causal association. ${ }^{65}$ Several cross-sectional and prospective studies have now confirmed that there is a significant association between SDB and ischemic heart disease, ${ }^{66,67}$ myocardial infarction ${ }^{68}$ and congestive heart failure. ${ }^{5,69,70} \mathrm{~A}$ significant relationship between SDB and ischemic stroke also exists. ${ }^{62,71} \mathrm{~A}$ growing body of evidence suggests that SDB is associated with metabolic dysfunction. Metrics of SDB severity such as $\mathrm{AHI}$ and degree of hypoxaemia are associated with the degree of insulin resistance, glucose intolerance and type 2 diabetes mellitus independent of potential confounders including obesity. ${ }^{72,73}$

The degree of SDB also appears to be a predictor of cardiovascular risk. While severe OSA (AHI > $30 / \mathrm{hr}$ ) is strongly associated with increased mortality, stroke and cardiovascular disease, the risk from moderate OSA ( $\mathrm{AHI} 15-30 / \mathrm{hr}$ ) is uncertain, particularly if the ODI is low. There is no conclusive evidence of increased cardiovascular risk from mild OSA $(\mathrm{AHI}<15 / \mathrm{hr}) .{ }^{74}$

Sleep is chronically disturbed in untreated SDB. As a consequence, individuals with SDB may experience variable degrees of cognitive and performance deficits such as difficulties with cognitive processing, inability to concentrate, memory problems, poor executive abilities and mood changes. ${ }^{75,76}$ The deficits experienced by those with SDB are associated with decreased work performance ${ }^{77,78}$ lower general health status and diminished quality of life. ${ }^{79,80}$ Sleep loss, sleepiness and resultant neurobehavioural performance deficits in SDB sufferers represents a significant risk to driving safety. Compared to normal controls, non-commercial drivers with untreated sleep apnoea are 2 to 3 times more likely to experience a motor vehicle accident, with successful treatment of sleep apnoea improving driver performance. ${ }^{81}$

\subsubsection{Treatment of Sleep-Disordered Breathing}

Continuous positive airway pressure (CPAP) is the gold standard treatment for moderate to severe OSA. CPAP acts as a pneumatic splint to prevent collapse of the pharyngeal airway. ${ }^{82}$ CPAP therapy utilises a machine specifically designed to deliver a constant flow of air pressure, delivered via a nasal or oronasal mask. Although CPAP is the most effective way to treat OSA many patients have poor adherence, often due to tolerance issues with the air pressure or the mask interface.

Treatment with CPAP may ameliorate the negative health outcomes associated with OSA. Two recent meta-analyses described modest but statistically significant reductions in diurnal and nocturnal BP in patients with OSA treated with CPAP therapy. ${ }^{83,84}$ In a large prospective cohort study over 10 years, patients on CPAP therapy had a lower incidence of fatal and nonfatal cardiovascular events compared to those with untreated severe OSA. ${ }^{85}$ On the other hand, a large Australian-based study showed that CPAP therapy did not prevent cardiovascular events in patients with moderate to severe OSA and established cardiovascular disease, however health-related quality of life and mood was improved. ${ }^{86}$ 
CPAP treatment has been shown to reverse endothelial dysfunction that was related to severity of OSA. ${ }^{87}$

A recent review highlighted how CPAP can have a positive effect on diabetes mellitus through various metabolic parameters, however negative results were also reported. ${ }^{88}$ Adherence to CPAP therapy for 6 months has been shown to improve quality of life measures, daytime sleepiness and symptoms associated with OSA. ${ }^{89}$ Motor vehicle crash risk among drivers with OSA is reduced following CPAP treatment, with simulated driving performance improving within 2 to 7 days of initiating treatment. ${ }^{90} \mathrm{~A}$ meta-analysis recently found that CPAP treatment has a small significant effect on improving attention but not other cognitive domains. ${ }^{91}$

Data on the benefit of CPAP therapy in various conditions may differ for a number of reasons - different definitions and methods to diagnose SDB, variable adherence to CPAP therapy and treatment duration as well as management of confounding variables and comorbidities. A recent meta-analysis showed that $\mathrm{BP}$ reductions following CPAP treatment were evident only in studies with younger patients, greater degrees of daytime hypersomnolence and more severe OSA. ${ }^{84}$ Regardless, given the sizable potential health risks associated with untreated SDB and the postulation that pregnancy may give rise to a higher incidence of this treatable condition, further investigation into the incidence and consequences of SDB during pregnancy is warranted.

\section{$1.4 \quad$ Sleep-Disordered Breathing in Pregnancy}

Pregnancy is associated with many physiological changes that may promote SDB due to their resemblance to known anatomical risk factors. Firstly, progressive gestational weight gain along with upper airway oedema may reduce upper airway dimensions ${ }^{92}$ and predispose women to apnoeic respiratory events during sleep. During pregnancy, functional residual capacity is often reduced, $, 93,94$ due to elevation of the diaphragm from the expanding uterus. It has also been suggested that the increase in the hormone relaxin culminates in increased upper airway collapsibility and decreased upper airway calibre, ${ }^{92,95}$ and changes in the airway mucosa results in nasopharyngeal oedema. ${ }^{96}$ Increased progesterone during pregnancy may in fact be protective against apnoeic respiratory events due to its impact on heightened respiratory drive, ${ }^{97}$ which protects against upper airway occlusion by enhancing responsiveness of upper airway dilator muscles to stimuli during sleep. ${ }^{98}$ However, progesterone also increases diaphragmatic effort leading to greater negative inspiratory pressures at the level of the upper airway which may lead to an increased tendency for the upper airway to collapse during sleep. ${ }^{99}$ For these reasons, as pregnancy progresses episodes of partial or complete upper airway obstruction may cumulate and progress to SDB.

On the other hand, postural changes during sleep may help protect pregnant women from SDB as by late pregnancy most women avoid sleeping in the supine position, when SDB is typically most severe. 47,48 Obstructive events are often more common during REM sleep, a sleep stage which is often decreased during pregnancy. ${ }^{100,101}$ 


\subsubsection{Prevalence of Sleep-Disordered Breathing in Pregnancy}

The prevalence of SDB during pregnancy remains unknown. Due to the methodological constraints of performing sleep studies on high numbers of pregnant women, current SDB prevalence estimates have been predominantly based on self-report data.

\subsubsection{Self-Reported Snoring and Sleep-Disordered Breathing Symptoms}

Studies demonstrate that as many as 12 to $41 \%$ of women report frequent snoring by the third trimester of pregnancy, ${ }^{6,92,102-104}$ compared with only 4 to $17 \%$ of non-pregnant women of similar age.6,92,102 Symptoms of SDB such as loud snoring, snorting, or gasping and apnoeas reportedly increase as pregnancy progresses. ${ }^{105}$

Gathering prevalence data based on subjective questionnaires is suboptimal in this population. A number of studies have now demonstrated that the most commonly used questionnaires to characterise SDB in pregnancy poorly predict a diagnosis of SDB. ${ }^{106-109}$ The Berlin Questionnaire, ${ }^{110}$ Epworth Sleepiness Scale (ESS) ${ }^{111}$ and Multivariate Apnea Prediction Index ${ }^{112}$ have all been validated in nonpregnant populations but have so far proven inappropriate and unreliable as a screening tool in pregnancy. For example, the Berlin Questionnaire demonstrated poor sensitivity and specificity of 0.35 and 0.64 respectively in one study, ${ }^{107}$ whereas another study demonstrated adequate sensitivity but very poor specificity ( 0.87 and 0.32 respectively) for detecting an $\mathrm{RDI}>5 / \mathrm{hr} .{ }^{108}$

\subsubsection{Objective Data for Sleep-Disordered Breathing Prevalence in Pregnancy}

As described in Section 1.3.1, objectively-measured SDB can be categorised by severity with the AHI or RDI. AHI has historically been the standard measure, however with the introduction of scoring of RERAs in $2007,38 \mathrm{RDI}$ can be used as a more widely encompassing measure of SDB. RERAs can appear without a significant number of apnoeas and hypopnoeas, in what is referred to as UARS. ${ }^{113}$ RERAs are presumed to have a similar pathophysiology to obstructive apnoeas and hypopnoeas and are believed to be as much of a risk factor for symptoms of unrefreshing sleep, daytime somnolence and fatigue. ${ }^{114}$ For example, patients with UARS (defined as a normal AHI but increased RDI) had worse sleep quality and more fatigue than patients with mild OSA (AHI $\geq 5$ and $\leq 15$ events/hour). ${ }^{115}$ There is also evidence that in the absence of apnoeas and hypopnoeas, repetitive increased in BP can occur as a result of increased airway resistance during sleep, and that treatment of UARS can help to control high BP. ${ }^{116}$

Gender differences in SDB disease manifestation are thought to exist. Two clinical investigations found that although there was a male predominance in both conditions, more women were diagnosed with UARS than with OSA. ${ }^{117,118}$ Women with OSA are also more likely to have partial airway obstruction than complete obstruction and to have a shorter duration of respiratory events. ${ }^{119}$ For these reasons, it could be argued that RDI rather than $\mathrm{AHI}$ is a more appropriate measure of SDB in women, including during pregnancy. 
To date, only a small number of studies have used objective sleep monitoring during uncomplicated pregnancy. One of the earliest PSG studies of SDB during pregnancy was performed by Guilleminault and colleagues. ${ }^{120}$ Overnight PSG recording in a group of 26 women showed that although no participant demonstrated an $\mathrm{AHI}$ of $>5 / \mathrm{hr}$, the snorers presented two features not seen in the nonsnorers. Firstly, 'crescendos' consisted of progressive increases in respiratory effort, terminated by an abrupt drop in effort and typically an EEG micro-arousal. The second pattern was termed 'abnormal sustained effort' and presented as an abrupt increase in respiratory effort continuing for successive breaths, also terminating variably with an EEG change. The mean 24-hr systolic and diastolic BP was significantly higher for the 'abnormal breathing group', suggesting that lesser degrees of SDB may also have important consequences.

Another study focusing on inspiratory airflow limitation during sleep found that women in the third trimester of pregnancy spend $15 \%$ of the night with limited airflow, compared to less than $5 \%$ for women in the first and second trimester, and non-pregnant women. None of the 60 women displayed evidence of clinically significant SDB. ${ }^{121}$

Four large cohort studies focusing on OSA prevalence during high-risk pregnancy have recently been published, with three of these being longitudinal in design. ${ }^{7,122,123}$ Pien et al. ${ }^{7}$ performed PSG on 105 women in the first and third trimester of pregnancy, with recruitment stratified by body mass index (BMI) class to enrich the sample for subjects likely to develop SDB. First trimester women had a mean AHI of 2.1 (median 1.1) compared to a mean AHI of 3.7 (median 1.5) in the third trimester, demonstrating that SDB events increase during pregnancy but the magnitude of the increase is small. Proportionally, $10.5 \%$ of women had SDB ( $A H I \geq 5$ ) in the first trimester and $26.7 \%$ had SDB in the third trimester; only 4 of these had moderate SDB (AHI 15-29) and 1 severe (AHI $\geq 30$ ). Women with third trimester OSA were mostly obese $\left(\mathrm{BMI} \geq 30 \mathrm{~kg} / \mathrm{m}^{2}\right)$, with $40 \%$ of the obese women having OSA by the third trimester compared to $14.5 \%$ of normal or overweight $\left(\mathrm{BMI}<30 \mathrm{~kg} / \mathrm{m}^{2}\right)$ women. Higher $\mathrm{BMI}$ and maternal age were significant determinants for developing OSA in the third trimester of pregnancy.

Another group of 128 high-risk pregnant women with obesity, chronic hypertension, pre-gestational diabetes, history of PE and/or a twin gestation, completed home overnight evaluation with an abbreviated sleep monitor called the Watch-PAT100, between 6-20 weeks and again in the third trimester. ${ }^{122}$ In early pregnancy, $21 \%, 6 \%$ and $3 \%$ of women had mild (AHI 5-14.9), moderate (AHI 1529.9 ) or severe $(\mathrm{AHI} \geq 30$ ) SDB respectively; these frequencies increased to $35 \%, 7 \%$ and $5 \%$ in the third trimester. About $27 \%(n=34)$ experienced a worsening of SDB by the third trimester; 26 of these were cases of new-onset SDB while the other 8 had SDB in early pregnancy that worsened in severity. The incidence of new onset SDB was $20 \%$, with the majority being of mild severity. ${ }^{122}$

Following this, the same group were involved in the publication of the most recent study of SDB prevalence in pregnancy which enrolled over 3,000 nulliparous women. ${ }^{123}$ Use of the Watch-PAT100 device in early (6 - 15 weeks of gestation) and mid-pregnancy (22 - 31 weeks of gestation) found the 
prevalence of SDB (defined as an $\mathrm{AHI} \geq 5$ ) was $3.6 \%$ and $8.3 \%$ respectively. The majority of SDB cases were mild; only nine participants had an $\mathrm{AHI} \geq 15$ in early pregnancy and all of these women were obese.

The study by Louis et al. ${ }^{124}$ involved overnight monitoring with a limited channel recording device on 175 women with a pre-pregnancy BMI of $>30 \mathrm{~kg} / \mathrm{m}^{2}$. OSA prevalence (defined as an $\mathrm{AHI}>5$ ) in this study was $15.4 \%$, with those with OSA having a higher mean BMI.

Edwards et al. ${ }^{125}$ investigated 10 non-obese women with severe SDB during the third trimester and found that the degree of disease reduced markedly by 3 months post-partum, to mild-moderate levels. Although not directly providing prevalence data, this study highlights how late pregnancy may increase SDB severity.

From the limited number of available studies objectively measuring SDB during healthy, uncomplicated pregnancy, it appears that OSA is relatively uncommon. However milder forms of SDB such as airflow limitation or upper airway resistance may have some significance. Higher risk pregnant women on the other hand, especially those with obesity, are much more likely to be diagnosed with SDB, however the level of disease is typically minor with very few cases of severe SDB reported.

\subsubsection{Relevance of Sleep-Disordered Breathing in Pregnancy}

Why would the development of SDB during pregnancy be of clinical concern? As previously discussed, cyclical episodes of airway obstruction with intermittent hypoxia can result in sympathetic activation, oxidative stress and inflammation, resulting in endothelial dysfunction. Consequently, snoring and SDB are associated with a number of health risks, with the strongest evidence linking SDB to cardiovascular health, particularly hypertension. ${ }^{4}$ In the non-pregnant population, SDB confers a 3-fold increase in risk of hypertension independent of other risk factors, ${ }^{126}$ and perimenopausal women with SDB have a higher prevalence of hypertension and antihypertensive medication use. ${ }^{127}$ Several studies have shown that habitual snoring, considered to be on the milder end of the SDB spectrum, is also a determinant of risk of arterial hypertension in both men and women. ${ }^{128,129}$

Hypertensive disorders in pregnancy are common but very serious conditions associated with increased maternal and fetal morbidity and mortality. Identification of potentially modifiable risk factors for this condition, such as SDB and its pathophysiological consequences, may lead to better health outcomes for these women.

\subsection{Hypertensive Disorders of Pregnancy}

\subsubsection{Gestational Hypertension}

GH is characterised by the new onset of hypertension after 20 weeks gestation and affects up to $10 \%$ of pregnancies. ${ }^{130,131}$ Hypertension during pregnancy is defined as systolic BP greater than or equal to 
$140 \mathrm{mmHg}$ and or/ diastolic BP greater than or equal to $90 \mathrm{mmHg}$, with these measurements confirmed by repeated readings over several hours. ${ }^{132}$ Severe hypertension in pregnancy is defined as a systolic BP greater than or equal to $170 \mathrm{mmHg}$ and/or diastolic BP greater than or equal to $110 \mathrm{mmHg}$. This represents a level of BP above which cerebral autoregulation is overcome in normotensive individuals. ${ }^{132}$

\subsubsection{Preeclampsia}

PE is a multi-system disorder unique to human pregnancy characterised by hypertension and involvement of one or more other organ systems and/or the fetus. It is estimated to complicate $2-8 \%$ of all pregnancies. ${ }^{133}$ According to the International Society for the Study of Hypertension in Pregnancy (ISSHP), a clinical diagnosis of PE can be made when hypertension arises after 20 weeks gestation and is accompanied by new onset of proteinuria, or one or more of renal, haematological, liver or neurological involvement, or uteroplacental dysfunction. ${ }^{134}$ For research purposes, the definition of PE is new hypertension after 20 weeks gestation returning to normal post-partum, and properly documented proteinuria (confirmed by spot urine protein/creatinine ratio $\geq 30 \mathrm{mg} / \mathrm{mmol}$ ). ${ }^{134}$

The exact cause of PE remains unknown, however it is believed to be caused by the presence of the placenta or the maternal response to placentation (see Figure 1.3). ${ }^{3}$ In PE, the placentation process is aberrant. The invasion of the embryo-derived cytotrophoblasts is incomplete, with cells present only in the superficial layers of the maternal uterine wall. ${ }^{135}$ The spiral arteries fail to be invaded or remodelled, resulting in constricted, high-resistance vessels. ${ }^{136}$ This adaptive failure results in substantially reduced placental blood supply in the preeclamptic woman. ${ }^{137}$

The malperfusion of the placenta is thought to result in placental oxidative stress and the release of a complex mix of factors, including markers of oxidative stress, pro-inflammatory cytokines, apoptotic debris and angiogenic regulators into the maternal circulation. ${ }^{138}$ In particular, the release of antiangiogenic factors such as soluble fms-like tyrosine kinase-1 (sFlt-1) and soluble endoglin (sEng) has been implicated as the cause of the endothelial dysfunction resulting in many of the maternal features of $\mathrm{PE}$, such as hypertension, proteinuria, liver and cerebrovascular involvement. PE can also manifest clinically as a fetal syndrome, where poor placentation and ischemia result in fetal growth restriction (FGR), reduced amniotic fluid and abnormal fetal oxygenation. ${ }^{3}$

Up to $25 \%$ of women who initially present with GH will be in the process of developing PE but have not yet developed other manifestations. Any women presenting with new hypertension after 20 weeks gestation should be assessed for signs and symptoms of PE. If features of PE are detected, admission to hospital is generally indicated. ${ }^{132}$

$\mathrm{PE}$ is a progressive disorder that will inevitably worsen if pregnancy continues. No therapy exists to ameliorate the placental pathology or alter the pathophysiology of PE. Delivery is the only definitive management and is generally followed by resolution within a few days. Treatment of hypertension in 
$\mathrm{PE}$ is intended to prevent cerebral haemorrhage and eclampsia (seizures or convulsions which can be followed by coma) and perhaps delay progression of proteinuria and delivery. This prolongation of pregnancy in the presence of PE carries no benefit for the mother but is desirable at early gestations to improve the fetal prognosis. ${ }^{132}$

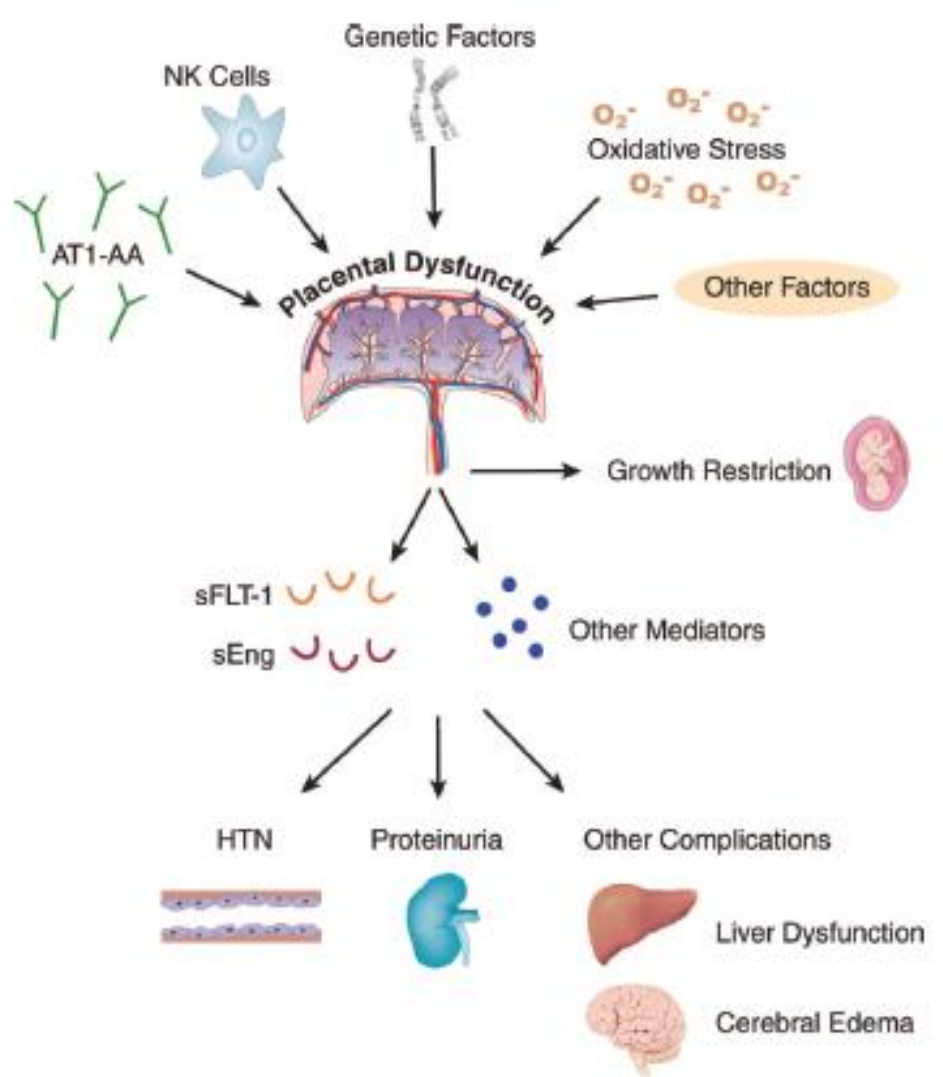

Figure 1.3 Summary of the pathogenesis of preeclampsia. Angiotensin II Type I Receptor Activating Autoantibodies (AT1-AA), immunologic factors, oxidative stress, and other factors may cause placental dysfunction, which in turns leads to the release of antiangiogenic factors (such as sFlt-1 and soluble endoglin [sEng]) and other inflammatory mediators to induce preeclampsia. NK indicates natural killer; HTN, hypertension. ${ }^{136}$

\subsubsection{Chronic Hypertension}

Essential hypertension is defined by a BP $>140 \mathrm{mmHg}$ systolic and/or $>90 \mathrm{mmHg}$ diastolic confirmed before pregnancy or before 20 completed weeks gestation without a known cause. Chronic hypertension can also have a secondary cause, such as kidney disease, renal artery stenosis, systemic disease, and endocrine disorders. Some women with apparent essential hypertension may have white 
coat hypertension. This is defined as hypertension in a clinical setting with normal BP away from this setting when assessed by 24-hour ambulatory BP monitoring or home BP monitoring. ${ }^{132}$

\subsubsection{Preeclampsia Superimposed on Chronic Hypertension}

Superimposed PE is diagnosed when a woman with chronic hypertension develops one of more of the systemic features of PE after 20 weeks gestation. Existing hypertension prior to pregnancy is a strong risk factor for the development of PE. ${ }^{139}$ Those with mild hypertension have a risk of PE of up to $20 \%$, but the risk increases up to $50 \%$ with severe hypertension. ${ }^{140}$ Worsening or accelerated hypertension should increase surveillance for PE but is not diagnostic.

\subsubsection{Maternal and Fetal Outcomes associated with Hypertensive Disorders of Pregnancy}

Hypertensive disorders were responsible for $14 \%$ of maternal deaths worldwide and was the second most common direct cause between 2003 - 2012. ${ }^{141}$ Although most maternal deaths occur in developing countries, the proportion of direct obstetric deaths associated with PE and eclampsia in high-income countries is similar to that in low and middle-income countries (10-15\%). ${ }^{133} \mathrm{PE}$ is also one of the most common maternal complications associated with late fetal death and early neonatal death. ${ }^{142}$

Adverse perinatal outcomes are generally increased in women with all subcategories of hypertensive disease in pregnancy as compared to normotensive women. ${ }^{143}$ In particular, there is considerable data showing that $\mathrm{PE}$ is associated with an increased risk of adverse pregnancy outcomes, whereas the risks in women with $\mathrm{GH}$ are less clear. ${ }^{144}$

In a large multicentre study involving over 2000 nulliparous women, ${ }^{145}$ the outcome of pregnancies complicated by either PE or GH was compared with that of uncomplicated pregnancies. Overall, the rate of perinatal complications including prematurity, low birthweight, FGR, placental abruption and perinatal mortality was higher only in the PE group, while the rate of these complications in the GH group was similar to that observed in uncomplicated pregnancies. Similarly, another large retrospective study showed that the rates of FGR, placental abruption and low Apgar scores were higher only in the mild PE group and not in the GH group, compared to normotensive women. ${ }^{146}$ However, women with $\mathrm{GH}$ as well as PE had an increased risk for postpartum haemorrhage and intensive care unit (ICU) admission, with the neonates of both groups of women being more likely to suffer from respiratory distress syndrome and pneumonia. A recent study by Shen et al. ${ }^{147}$ had similar results. Compared to normotensive women, PE significantly increased the risk of adverse outcomes including caesarean section, placental abruption, small for gestational age, preterm birth, and decreased 5 min Apgar scores. GH significantly increased the risk of preterm birth only.

The increase in adverse outcomes is also greater in those with superimposed PE. ${ }^{148}$ Among patients who developed severe PE, those with prior chronic hypertension were found to have a significantly 
higher incidence of perinatal mortality $(32 \%$ vs. $7.7 \%, p<.001)$, with more frequent placental abruption and small for gestational age infants. ${ }^{149}$

The severity of GH appears to dictate the severity of adverse pregnancy outcomes. Whereas outcomes of women with mild GH have been shown to be similar to those of uncomplicated pregnancies, women with severe GH have been shown to have significantly higher rates of clinical complications including placental abruption, FGR, neonatal morbidity and maternal renal dysfunction. ${ }^{150}$ Women with severe $\mathrm{GH}$ have also been shown to have higher rates of preterm delivery and small for gestational age infants when compared to those with mild GH and even mild PE. ${ }^{151}$

In PE, gestational age at disease onset is a strong predictor of fetal prognosis. A large cohort study of all singleton deliveries in Washington State, USA, over a five-year period compared both fetal and maternal outcomes for early ( $<34$ weeks) and late-onset ( $\geq 34$ weeks) PE to women without the disease. Early- but not late-onset PE conferred a high risk of fetal (adjusted odds ratio [aOR], 5.8 vs. $\mathrm{aOR}, 1.3$ ) and neonatal death (aOR, $11.4 \mathrm{vs.} \mathrm{aOR,} \mathrm{1.1).} \mathrm{Liveborn} \mathrm{infants} \mathrm{of} \mathrm{mothers} \mathrm{with} \mathrm{early-onset}$ $\mathrm{PE}$ also had higher odds of severe neonatal morbidity, neonatal intensive care unit (NICU) admission and small for gestational age compared with those born at the same gestation to mothers without PE, whereas late-onset PE was associated with significantly elevated rates of small for gestational age infants only. ${ }^{152}$ In terms of maternal outcomes, the rate of severe morbidity was highest among women with early-onset PE, with particularly high rates observed for cardiovascular, central nervous system (CNS) and respiratory morbidity, and acute renal and liver failure, compared with women without earlyonset disease. Rates of maternal morbidity were relatively lower among women with late-onset PE. ${ }^{153}$ Another study of early onset PE ( $<34$ weeks gestation) confirms that adverse infant outcome (as defined by perinatal death or severe morbidity) was predominantly influenced by gestation age, with the highest proportions of pregnancies affected before 30 weeks of gestation. ${ }^{154}$

Gestational age at onset of $\mathrm{GH}$ appears most related to likelihood of progression to PE. For example, a study by Saudan et al. ${ }^{155}$ found that over $40 \%$ of those presenting with GH prior to 30 weeks gestation progressed on to develop PE. However, those diagnosed with $\mathrm{GH}$ after 36 weeks of gestation had only a $10 \%$ risk of developing PE.

Hypertensive disorders during pregnancy can also predispose to longer-term cardiovascular issues. Following pregnancies complicated by PE, women have a higher long-term risk of ischemic heart disease and stroke, venous thromboembolism and development of congestive heart failure. Both PE and GH are associated with an increased risk of chronic hypertension subsequent to the pregnancy. 156,157 


\section{6 Proposed Relationship between Sleep-Disordered Breathing and Hypertensive Disorders of Pregnancy}

PE and SDB appear to share biological pathways. It is biologically plausible that SDB could contribute to the development of hypertension or PE in pregnancy via the mechanisms outlined in Figure 1.4. The repeated episodes of hypoxia associated with SDB may (i) provoke placental ischaemia directly, (ii) contribute to hypertension through sympathetic activation, and (iii) contribute to endothelial dysfunction induced by the pro-inflammatory state. In turn, the upper airway oedema associated with PE may exacerbate the cascade.

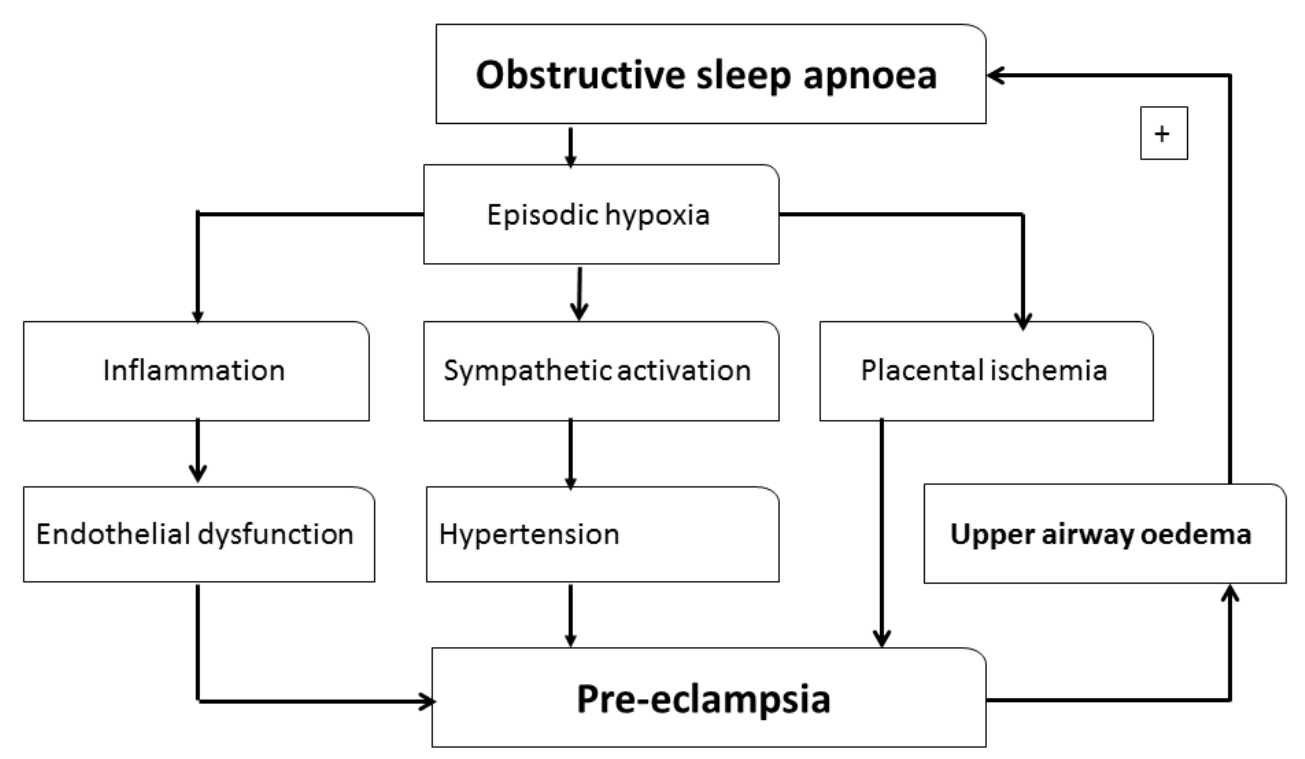

Figure 1.4 Potential interaction between SDB and hypertensive disorders of pregnancy ${ }^{49}$

$\mathrm{PE}$ is a multisystem disorder manifested by widespread endothelial dysfunction, committed by placental factors secreted into the maternal circulation when the placenta has been exposed to hypoxia or ischemia, inflammation or both. ${ }^{158}$ Additionally, increased circulating levels of anti-angiogenic factors, such as sFlt-1 and sEng, ${ }^{159}$ and reactive oxygen species ${ }^{160}$ are thought to be linked to the pathogenesis of PE. However, the exact cause of PE remains unknown. Outside of pregnancy, the intermittent hypoxic and hypercapnic events of SDB are known to result in sympathetic activation, oxidative stress and activation of inflammatory pathways that can lead to endothelial dysfunction. ${ }^{161}$ Individually or collectively, these are the proposed mechanisms by which SDB results in hypertension and may contribute to the development of PE. 


\subsubsection{Sympathetic Activation}

Levels of sympathetic activity have been shown to be elevated in women with PE. ${ }^{162}$ In SDB, the sympathetic nervous system appears to play a key role in cardiac pathophysiology. During sleep, because of activation of the peripheral and central chemoreflexes by hypoxia and hypercapnia, sympathetic activity increases. In the setting of apnoea, the inhibitory effect of the thoracic afferents is absent, thus resulting in further potentiation of sympathetic activation. The consequent vasoconstriction results in surges in BP. ${ }^{163}$ Sympathetic activity abruptly ceases at onset of breathing due to the inhibitory effect of the thoracic afferents (Figure 1.5). ${ }^{164}$ This may explain how chronic intermittent hypoxia causes hypertension. ${ }^{165}$

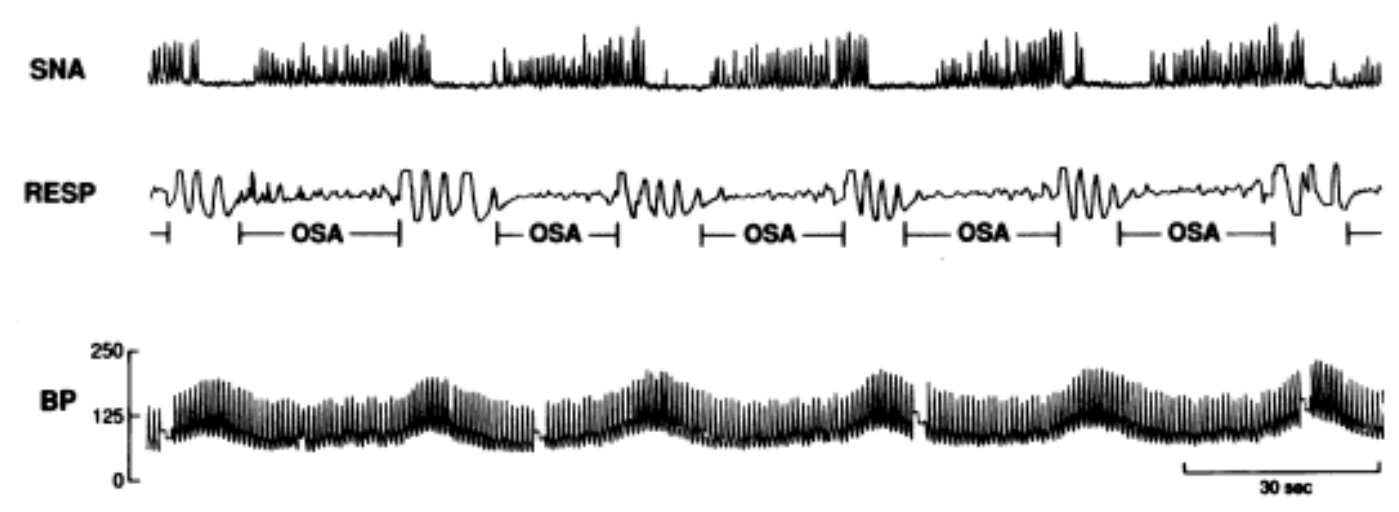

Figure 1.5 Recordings of sympathetic nerve activity (SNA), respiration (RESP) and blood pressure (BP) during 3 min of stage II sleep, showing incessant oscillations in blood pressure and sympathetic activity in response to repetitive apnoeas. ${ }^{164}$

Cortical arousal from sleep is associated with sympathetic activation leading to transient increases in heart rate, BP and muscle sympathetic nerve activity. ${ }^{166-168}$ Conditions such as SDB are characterised by frequent arousals across the night, where this leads to a sustained sympathetic influence on the cardiovascular system. ${ }^{163}$

Among pregnant women with SDB, increased sympathetic vasoconstrictor activity owing to apnoea, hypoxia and arousals during sleep may cause increases in peripheral vascular resistance, a key characteristic of PE. ${ }^{3}$ Such increases could lead to elevations in systemic arterial BP and reductions in maternal cardiac output, resulting in compromised uteroplacental blood flow, as is observed in PE. ${ }^{169-}$ 171 


\subsubsection{Oxidative Stress, Inflammation and Endothelial Dysfunction}

Hypoxaemia with reoxygenation may be analogous to ischemia with reperfusion, and reoxygenation may cause additional damage through further production of free radical species. Biochemical injury due to hypoxaemia-reoxygenation has considerable relevance for patients with SDB, where intermittent and sometimes profound alterations in the partial pressure of oxygen $\left(\mathrm{PO}_{2}\right)$ may occur hundreds of times during sleep. ${ }^{161}$ Intermittent hypoxia and the additional production of oxygen radicals has been proposed to be more harmful than sustained hypoxia in terms of its contribution to oxidative stress. ${ }^{172}$ Among pregnant women with SDB, it is possible that hypoxaemia and reoxygenation following apnoea contribute to the development of $\mathrm{PE}$, due to the generation of reactive oxygen species causing oxidative stress damage to cells and tissues. ${ }^{137,173}$

The increased levels of pro-inflammatory cytokines that occur in SDB are another important potential contributor to the causal pathway in PE. The oxidative stress associated with SDB can increase inflammatory cytokines and activation of endothelial cells, leading to endothelial dysfunction. ${ }^{174}$ The non-pregnant literature suggests a significant increase in inflammatory markers such as TNF- $\alpha$, CRP and IL- 6 in patients with SDB compared with controls, ${ }^{54,56,175,176}$ which decrease back to normal levels following treatment. ${ }^{56}$

An increase in inflammatory cytokines has also been shown in placental diseases such as PE. Plasma concentrations of cytokines including CRP, IL-6, TNF- $\alpha$, and 8-isoprostane have all been found to be significantly higher in women with PE than in healthy pregnant women. ${ }^{177,178}$ These inflammatory markers are increased in both early- and late-onset PE, and are markedly more elevated when PE is severe. ${ }^{177,179}$ Elevations of CRP are also present from the first trimester of pregnancy in women destined to develop PE and FGR. ${ }^{180}$ Early onset of PE typically features inadequate and incomplete trophoblast invasion of maternal spiral arteries ${ }^{181}$; inflammatory cytokines such as TNF- $\alpha$ are thought to interfere with trophoblast implantation. ${ }^{182,183}$

Endothelial dysfunction is the most common clinical manifestation in PE. ${ }^{3}$ Yinon et al. ${ }^{184}$ demonstrated that women with PE had a lower endothelial function index than normotensive controls, however they also had significantly higher levels of SDB. A weak negative correlation between RDI and endothelial function index was found, leading them to speculate that SDB contributes to the functional abnormality of blood vessels seen in PE. Benyo et al. ${ }^{185}$ showed that inducing hypoxia in the human placenta could lead to the elaboration of the inflammatory cytokines TNF- $\alpha$ and interleukin-1; cytokines that are capable of eliciting endothelial cell dysfunction.

\subsubsection{Angiogenic Markers}

Angiogenesis is the physiological process through which new blood vessels grow from existing vasculature $^{186}$; therefore anti-angiogenic factors inhibit this process. Excess circulation of placental antiangiogenic factors are thought to be responsible for producing systemic endothelial dysfunction, 
resulting in hypertension, proteinuria and the other systemic manifestations of PE. ${ }^{187}$ SFlt-1 and sEng, secreted by the placenta, are increased in the maternal circulation weeks before the onset of PE, with levels of sFlt-1 and the pro-angiogenic placental growth factor (PIGF) correlated with disease severity. ${ }^{188-191}$ Furthermore, the ratio of sFIt-1 to PIGF has been shown to be a better marker of PE than either measure alone, ${ }^{192-194}$ implying an imbalance of anti- and pro-angiogenic factors lead to PE. ${ }^{136}$

Anti-angiogenic proteins are released under hypoxic stress and secondary to the inflammatory state ${ }^{195}$; it is therefore feasible that SDB may result in higher levels in circulation. To date, serum sFlt-1 and sEng levels have not been clearly determined in patients with SDB, ${ }^{196}$ however one study has demonstrated significantly elevated plasma concentrations of sFlt-1, but not sEng, in normotensive patients with SDB compared to controls. ${ }^{197}$ Within a pregnant sample, Bourjeily and colleagues ${ }^{198}$ found that women with OSA had lower levels of PIGF compared to controls, and the ratio of sFIt-1/PIGF was significantly higher in OSA cases after adjustment for relevant confounders.

We recently reported a case study on a patient diagnosed with early-onset PE and severe SDB. ${ }^{158}$ After commencement of CPAP therapy for SDB, BP and proteinuria were improved. In addition, circulating levels of sFlt-1 were reduced by over $50 \%$ and remained low for 23 days. The ratio of sFlt-1 to PIGF also decreased, as did sEng. In this case, treatment of SDB resulted in improved clinical and angiogenic markers of $\mathrm{PE}$, implying a role for SDB in the pathogenesis of $\mathrm{PE}$ in this patient.

\subsection{Sleep-Disordered Breathing and Fetal Health}

As well as SDB linking to hypertensive disease in pregnancy, it is plausible that the intermittent hypoxia and hypercapnia, systemic inflammatory response and endothelial dysfunction may be an important contributor to fetal compromise. The potential interaction between SDB and acute or chronic fetal compromise is summarised in Figure 1.6.49 


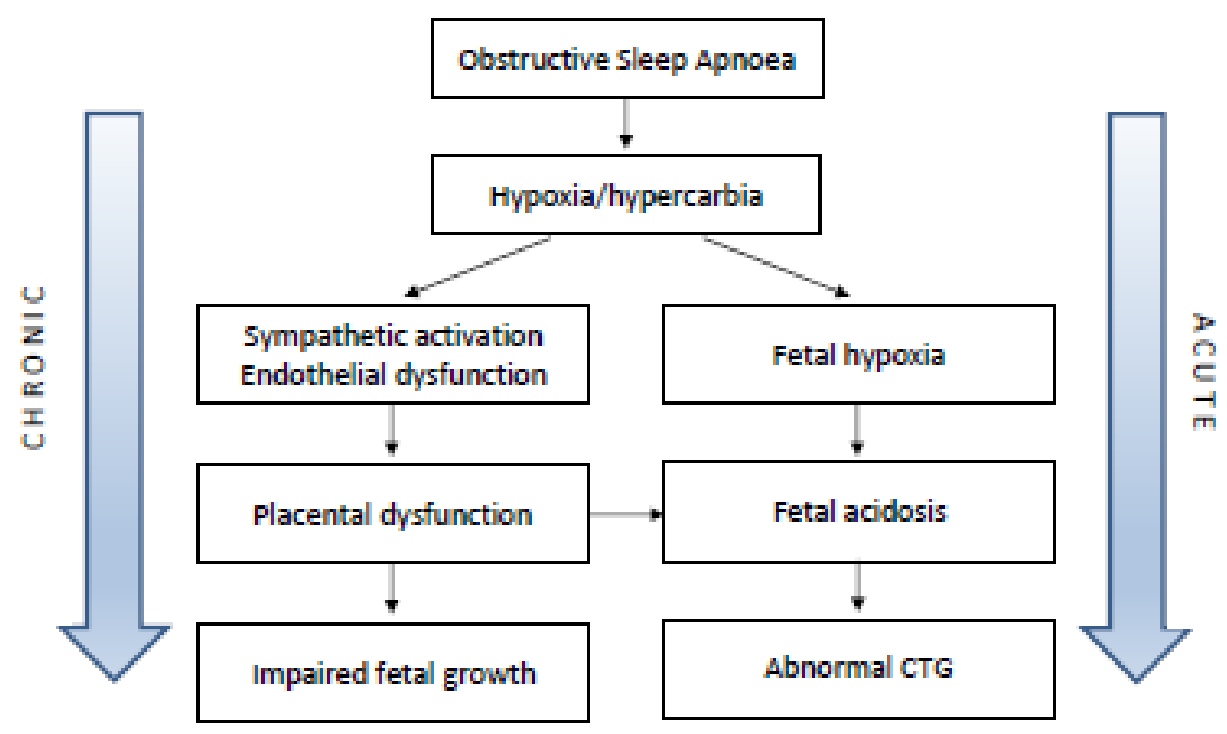

Figure 1.6 Potential interaction between SDB and acute and chronic fetal compromise. CTG = cardiotocogram. 49

\subsubsection{Markers of Impaired Fetal Growth}

Impairment of fetal growth may have maternal or placental contributors. Maternal contributors include hypertension 199,200 and conditions associated with hypoxia, ${ }^{201-203}$ whereas placental factors include impaired vascular perfusion ${ }^{204}$ or inflammatory activation ${ }^{205}$ that may contribute to impaired oxygen and nutrient exchange. Hypertension and peripheral vasoconstriction have been shown to be associated with reduced placental delivery to the fetus. ${ }^{206}$ It is plausible that, through these mechanisms, SDB may be a contributor to FGR, over and above that caused by hypertensive disease alone.

In general, insulin-like growth factor (IGF) is a hormone involved in mediating growth, differentiation, survival and metabolism in almost every organ of the body, ${ }^{207}$ and within pregnancy the IGF axis is an important regulator of placental development and fetal growth. ${ }^{208}$ Numerous studies have demonstrated the importance of IGFs actions for fetal growth and development, with birthweight positively correlated with insulin-like growth factor 1 (IGF-1) levels in cord blood in healthy newborns, ${ }^{209,210}$ whereas levels of IGF-1 are low in small for gestational age infants. ${ }^{211}$ There are numerous reports of increased maternal insulin-like growth factor binding protein 1 (IGFBP-1) and insulin-like growth factor binding protein 2 (IGFBP-2) levels in pregnancy complications such as FGR. ${ }^{209,212,213}$ In non-pregnant people with SDB, IGF-1 has been negatively correlated with parameters of apnoea such as AHI, ODI and minimum oxygen saturations, ${ }^{214,215}$ whereas treatment of SDB with CPAP leads to significant increases in IGF-1 concentrations, particularly in younger men. ${ }^{215,216}$ 
When comparing small for gestational age and appropriate for gestational age infants, there are significant differences seen in the concentration of adipocytokines, such as leptin, adiponectin, IL-6 and TNF- $\alpha .{ }^{217}$ Small for gestational age infants have significant elevations in IL-6, TNF- $\alpha$, and CRP in cord serum compared to appropriately grown controls, ${ }^{218}$ and higher TNF- $\alpha$ levels in maternal serum of FGR pregnancies. ${ }^{219}$ As mentioned earlier, OSA is also associated with elevations in these proinflammatory cytokines, ${ }^{54-57}$ with leptin levels being positively correlated to OSA severity. ${ }^{58}$

Levels of oxidative stress have also been found to be increased in patients with FGR. ${ }^{220}$ Bourjeily et al. ${ }^{221}$ showed that the second trimester markers of fetoplacental unit wellbeing, alpha feto-protein (AFP) and estriol (uE3), are reduced in women with SDB compared to controls, however only the reduction in uE3 levels persisted after correction for maternal BMI.

\subsubsection{Measurement of Impaired Fetal Growth - Ultrasound}

In many countries, systematic "routine" ultrasonography is recommended in pregnancy in order to gain information regarding fetal health and well-being. ${ }^{222}$ Measurement of fetal size is based on ultrasound biometry, typically calculated using a formula based on measurements of head size, abdominal circumference and femur length. ${ }^{223}$ Estimated fetal weight (EFW) based on these calculations is then compared to population-based weight centiles to determine fetal growth.

A newer method of monitoring fetal growth is the use of customised birthweights to develop intrauterine growth curves for individual assessment of growth. Customised growth curves adjust for physiological factors known to affect fetal size, such as fetal gender, maternal weight and height, and ethnicity. ${ }^{224}$ These customised growth curves appear to be better than population curves at identifying small fetuses at risk of increased perinatal complications. ${ }^{225-227}$

Given that perinatal weight correlates with neonatal survival, ${ }^{222}$ fetal weight estimates commonly play a pivotal role in management decisions. Growth failure can be defined in several ways. Fetal growth restriction (FGR) is a term used to describe a pathological limitation to the growth of the fetus, implying a failure to reach a given size potential due to adverse genetic or environmental factors, ${ }^{228}$ and is defined as an EFW of below the $10^{\text {th }}$ percentile for gestational age on a standardised population growth curve. Small for gestational age is a term used for the neonate, defined as a birthweight of below the $10^{\text {th }}$ percentile, and intrauterine growth restriction (IUGR) refers to FGR with a small placenta and oligohydramnios. ${ }^{229}$ Growth restricted infants have higher risks of neonatal complications such as birth hypoxia, ${ }^{230}$ and growing evidence suggests that there are long-term metabolic consequences of FGR, such as increased risks of insulin resistance and cardiovascular complications. ${ }^{231}$ In addition to fetuses that have true FGR, about $70 \%$ of small for gestational age infants are healthy but constitutionally small fetuses that have a lower risk of abnormal perinatal outcomes. ${ }^{200,232}$ These babies characteristically grow at a constant speed that usually parallels a specific percentile throughout pregnancy, and typically have a normal postnatal outcome. ${ }^{229}$ Other measures of fetal well-being such as amniotic fluid volume, fetal arterial and venous Doppler measurements (blood flow velocity) and fetal heart rate, as well as 
charting growth velocity, can help distinguish the constitutionally small but well fetus from the compromised one. 228

Maternal risk factors for FGR include maternal hypoxia, ${ }^{201-203}$ along with hypertensive diseases of pregnancy ${ }^{233}$ hence the inclusion of fetal growth as an outcome of this study. All forms of hypertensive disease increase the incidence of FGR by two to threefold as a result of reduced uteroplacental perfusion - the interaction between maternal and fetal circulations in the placenta is fundamental for adequate exchange of nutrients and oxygen.200 Maternal SDB has also been associated with histopathologic evidence of chronic placental hypoxia and uteroplacental underperfusion, suggesting that SDB during pregnancy is associated with fetoplacental hypoxia, ${ }^{234}$ that may potentially impact fetal growth.

\subsubsection{Fetal Hypoxia/Acidosis}

Fetal hypoxia, which can be caused by inadequate maternal blood gas concentrations or inadequate uterine blood supply, may lead to fetal acidosis. ${ }^{235}$ Even small declines in maternal oxygenation can endanger oxygen delivery to the fetus, and any cause of maternal hypercapnia quickly leads to fetal respiratory acidosis. ${ }^{236}$ Especially if prolonged for days or weeks, chronic acidosis is associated with significant morbidity and mortality with potential long term damage, ${ }^{235}$ such as impaired neurodevelopment. 237

In situations where severe hypoxia and acidosis are suspected, one non-invasive method of assessment is fetal heart rate monitoring (cardiotocography - CTG). In animal models, exposure to intermittent hypoxia during pregnancy leads to FGR, fetal bradycardia and decreased fetal breathing movements. ${ }^{238,239}$ Fetal heart rate decelerations and accompanying acidosis have been recorded during maternal apnoeic episodes with oxygen desaturation in humans, ${ }^{240,241}$ however this hasn't been a consistent finding. ${ }^{107,242}$

Increased fetal erythropoiesis manifested by elevated circulating nucleated red blood cells (nRBCs) has been found in pregnancies complicated by fetal hypoxia. ${ }^{243}$ Both erythropoietin (EPO) and IL-6 mediate elevation of circulating nRBCs. The intermittent hypoxia and systemic inflammation elicited by SDB has been hypothesised to affect fetal erythropoiesis during pregnancy. ${ }^{206}$ Within a group of pregnant women, Tauman and colleagues 206 found that cord blood levels of circulating nRBCs, EPO and IL-6 were elevated in reported habitual snorers compared with non-snorers, suggesting that this marker of SDB is associated with subtle alterations in markers of fetal wellbeing.

\subsubsection{Measurement of Fetal Hypoxia/Acidosis - Cardiotocography}

Fetal heart rate analysis is the prime means by which a fetus is evaluated for adequacy of oxygenation.244 A cardiotocograph (CTG) is a continuous electronic record of the fetal heart rate obtained via an ultrasound transducer placed on the mother's abdomen. A second transducer is placed 
on the mother's abdomen over the uterine fundus to record simultaneously the presence of any uterine activity. CTG is often used in maternity care, both in the antepartum and postpartum periods. ${ }^{245}$ Widely recognised antenatal indications for fetal heart rate monitoring include decreased fetal movements, clinical FGR, and maternal hypertension including PE; where fetal heart rate may be affected due to reduced fetal reserves or reduced placental function. The term 'electronic fetal monitoring' is sometimes used synonymously with CTG monitoring, however can also refer to fetal electrocardiograph or fetal pulse oximetry. ${ }^{246}$

A normal antenatal CTG will demonstrate a baseline fetal heart rate of 110-160 bpm, normal baseline variability, reactivity and no decelerations (Figure 1.7). The presence of normal baseline variability (three to five cycle per minute fluctuations around the baseline, in the range of 5-25 bmp in amplitude) is the most important feature of the CTG in terms of fetal wellbeing, as this reflects a balanced sympathetic and parasympathetic input from the cardio-regulatory centre and represents an adequately oxygenated CNS. ${ }^{247}$ Reactivity is defined as the presence of two or more accelerations, or transient increases in fetal heart rate as a fetal response to stimulation, within a twenty minute period, and is another hallmark of fetal wellbeing and oxygenation. ${ }^{244}$

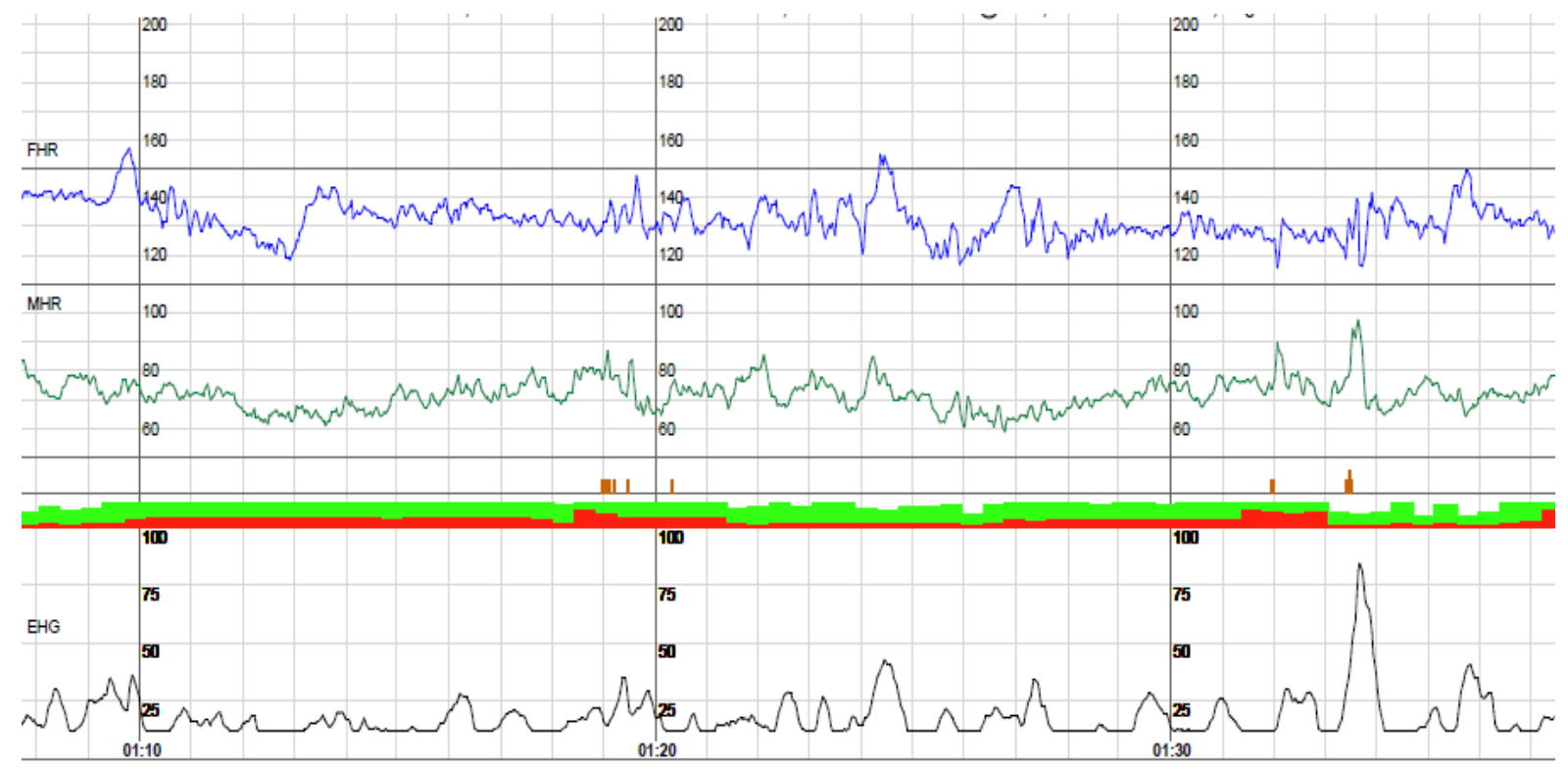

Figure 1.7 Normal antenatal cardiotocography (CTG) demonstrating a baseline fetal heart rate of approximately 125 beats per minute (bpm), normal baseline variability (5-15 bpm), fetal heart rate accelerations in response to fetal movement (elevation from baseline of at least $15 \mathrm{bpm}$ ) with no decelerations (spontaneous or provoked by uterine contractions). Heart rate $(\mathrm{bpm})$ is indicated on the vertical axes. Small horizontal divisions represent 1 min, with large divisions representing $10 \mathrm{~min}$. $F H R=$ fetal heart rate; $M H R=$ maternal heart rate; $E H G$ = Electro Hysterogram. 
Decelerations in fetal heart rate can be categorised into four types - early, variable, prolonged and late - with some variations. They are typically associated with uterine contractions ${ }^{244}$ and events such as cord compression during labour. In the antenatal period, well fetuses move regularly and will occasionally impeded umbilical cord blood flow, resulting in an isolated variable deceleration. In this situation, this does not mean the fetus is compromised. ${ }^{247}$ However, decelerations can be a sign of something pathological. Prolonged decelerations last for more than 90 seconds and less than 5 minutes, are caused by hypoxia and are reflective of the fetal condition and environment. Late decelerations are caused by contractions in the presence of hypoxia, and will start after the start of the contraction and return to baseline after the contraction has finished. ${ }^{248}$

Once a diagnosis of PE is made, it is mandatory to monitor the fetal condition. ${ }^{249}$ One study has shown that non-reassuring fetal heart rate on intrapartum CTG is more predominant in PE with either mild or severe proteinuria, with severe PE particularly associated with recurrent late decelerations. However, indicators of fetal distress on intrapartum CTG were not increased in GH compared to low risk pregnancies. ${ }^{250}$ Any fetus that is already compromised, such as with FGR, is also at an increased risk of developing late decelerations.

In the case of significant SDB with associated hypoxia, reduced maternal oxygen availability will rapidly impact on fetal oxygenation, as the availability of oxygen in the intervillous space is reduced. The initial response of the normal fetus to acute hypoxia or acidosis is a sudden drop from normal fetal heart rate, referred to as bradycardia (FHR of less than $110 \mathrm{bpm}$ ). ${ }^{251}$ Prolonged deceleration or bradycardia is considered to represent a prolonged stepwise decrease in fetal oxygenation, which can be due to maternal apnoea or a decrease in uterine blood flow, for example. The extent of the bradycardia and the time taken for the fetal heart rate to return to baseline is reflective of the degree of fetal hypoxia. ${ }^{244,248}$ A mild or accumulative hypoxia may also result in tachycardia (FHR baseline in $>160 \mathrm{bpm}$ ) through increased sympathetic innervation, in an attempt to correct the oxygen deficit. Bradycardia or tachycardia in the presence of other reassuring features such as accelerations or normal variability or no decelerations is not associated with hypoxia. ${ }^{248}$

Hypoxia is a relatively uncommon though important cause of reduced baseline variability. In the chronically hypoxic fetus this occurs through utero placental insufficiency and changes in biophysical activity, where in the more acutely hypoxic fetus this is due to alterations to the balance of sympathetic and parasympathetic CNS innervation. ${ }^{248}$ Accelerations commonly occur as a result of fetal movement. An absence of reassuring features such as accelerations may indicate an acutely hypoxic fetus, as it reduces unnecessary movements to reduce oxygen consumption and enhance its chance of survival. ${ }^{248}$ Compared to normotensive pregnancy, fetuses with preeclamptic mothers have been shown to display less spontaneous fetal heart rate accelerations. ${ }^{252}$ 
Figure 1.8 demonstrates a prolonged bradycardia on CTG at $32+4$ weeks gestation. This prolonged event was likely related to hypoxia following an episode of cord occlusion; the infant was delivered with a true knot in the cord.

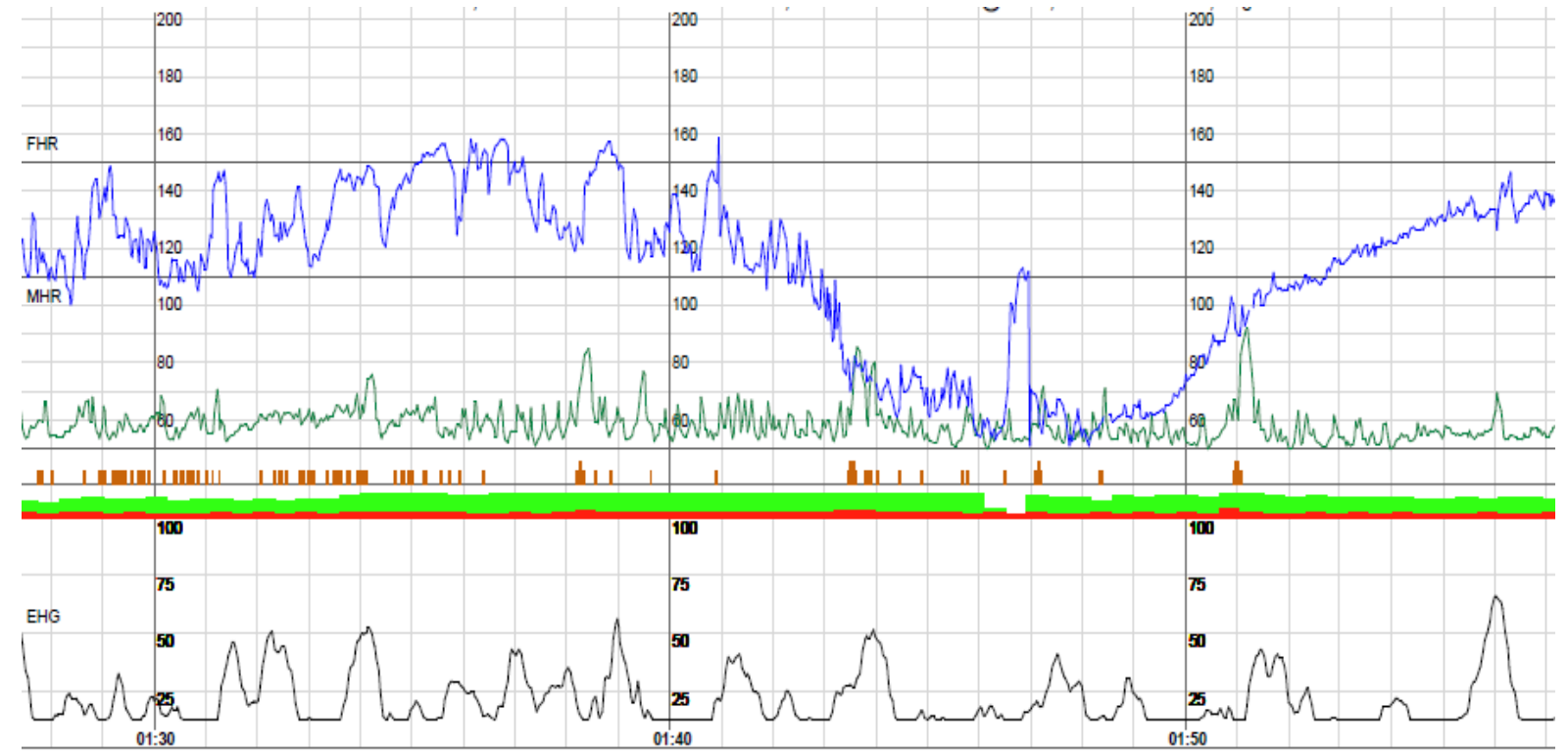

Figure 1.8 Example of prolonged fetal bradycardia on antenatal cardiotocography (CTG) at 32 weeks gestation. Heart rate (bpm) is indicated on the vertical axes. Small horizontal divisions represent $1 \mathrm{~min}$, with large divisions representing $10 \mathrm{~min}$. FHR = fetal heart rate; $M H R=$ maternal heart rate; $E H G=$ Electro Hysterogram.

\subsection{Sleep-Disordered Breathing and Hypertensive Disorders of Pregnancy - Current}

\section{Literature}

\subsubsection{Epidemiology Studies}

Several large cross-sectional studies have reported an increase in the prevalence of HDP among women reporting symptoms of SDB. GH and PE occur more frequently in pregnant women who report habitual snoring, ${ }^{6,103}$ and pregnant women scoring as high risk for SDB on predictive questionnaires have a higher incidence of PE. ${ }^{253,254}$ The association between SDB symptoms and PE persists even after adjusting for potential confounding variables such as age and BMI.9,104 Only two studies have failed to find a relationship between reported SDB symptoms and PE. ${ }^{255,256}$ However as discussed earlier, symptom reporting is a poor surrogate for diagnosis of SDB. 
A number of studies have employed objective techniques to quantify SDB to make comparisons between women with HDP and normotensive pregnant women. An initial study by Champagne et al.257 suggested a strong relationship between $\mathrm{GH}$ and the presence of SDB, which persisted after adjustment for maternal age and pre-pregnancy BMI (OR 7.5). It should be mentioned that in comparison to other PSG-based studies, this study had unusually high mean AHI values for both $\mathrm{GH}$ and normotensive females (38.6 \pm 36.7 vs. $18.2 \pm 12.2)$.

A similar study design using full PSG found that women with GH have a higher RDI on average than healthy pregnant women, with $53 \%$ of $\mathrm{GH}$ women having a significant level of SDB (RDI $\geq 5$ ) compared to only $12 \%$ of controls. ${ }^{8}$ There was a large significant discrepancy between the groups in BMI however $\left(37.4 \mathrm{~kg} / \mathrm{m}^{2} \pm 7.3\right.$ vs $28.7 \pm 4.4$ respectively), so that after adjustment for this strong SDB risk factor there remained no association between SDB and GH. Repeat testing of 18 women from this study (11 with GH and 7 healthy) 1-2 years postpartum, ${ }^{258}$ found that the women with $\mathrm{GH}$ had a decrease in mean $\mathrm{RDI}$ back to within normal levels, suggesting that the physiologic effects of pregnancy may have a pathologic role in the development of antepartum SDB in women with $\mathrm{GH}$.

Facco et al. ${ }^{259}$ performed overnight sleep evaluation with the Watch-PAT100 device on 20 preeclamptic inpatients and 20 normotensive control inpatients. Preeclamptic patients had higher mean indices of SDB (including AHI, RDI and ODI), however none of these differences reached statistical significance. As with other studies, BMI was markedly higher in the preeclamptic patients compared to controls. In a study which included chronic hypertension as well as GH and PE, ${ }^{260}$ the frequency of SDB for women with HDP was more than double that of normotensive controls ( $41 \%$ vs. $19 \%$ ). Again, the strong risk factor of BMI was not considered in any analysis, with only a mention that the normotensive controls had a significantly lower mean BMI pre-pregnancy $\left(23.7 \mathrm{~kg} / \mathrm{m}^{2}\right)$ compared to each hypertensive group $(P E=31.1, G H=34.1$, and chronic hypertension = 41.0). Also, a closer look at the data shows that the overall $\mathrm{AHI}$ values did not differ significantly between normotensives and any of the individual hypertensive groups; in particular the $\mathrm{GH}$ group had a similar prevalence of $\mathrm{AHI} \geq 5$ to the normotensive controls of $25 \%$, despite being obese on average. A study investigating SDB (as measured by ODI of $>4 \%$ ) as a cause of hypertensive disease in pregnancy also found no relationship, with not one of the 48 hypertensive women showing significant oxygen desaturation during sleep. ${ }^{261}$

Two studies by Louis et al. ${ }^{124,262}$ instead looked at the prevalence of PE among those with a diagnosis of OSA. A retrospective analysis of 57 pregnancies complicated by OSA, which was either diagnosed pre-pregnancy (at an unspecified time) or during pregnancy, compared maternal complications with normal weight and obese controls. ${ }^{262}$ All women diagnosed with OSA were recommended to use CPAP therapy however the authors state that compliance data was not available and therefore the degree of treatment undertaken by these participants is unknown. Results found that OSA patients had significantly more PE than normal weight women (19.3\% vs. $7.0 \%)$ but not obese controls of a similar mean BMI. The second study by Louis et al. ${ }^{124}$ focused on 175 obese pregnant women, but was limited by the use of a self-applied screening device for SDB that did not measure sleep staging or respiratory 
efforts. They found that after controlling for increased BMI and other relevant covariates, a diagnosis of OSA was still associated with an increased risk of developing PE (OR 3.55, 95\% Cl [1.1 - 11.3]). Worth noting is that both studies also found that chronic hypertension was significantly more common in the OSA groups compared to controls. ${ }^{124,262}$

The most recent and largest study to date by Facco et al. ${ }^{123}$ enrolled over 3,000 nulliparous women to undergo SDB monitoring in early and midpregnancy. Abbreviated home sleep tests were performed using a six-channel monitor that was self-applied by the participant; sleep data was not recorded and rather sleep time was estimated based on actigraphy. In early and midpregnancy, the adjusted odds ratios for PE when SDB was present were $1.94(95 \% \mathrm{Cl}$ [1.07 - 3.51]) and 1.95 (95\% Cl [1.18 - 3.23]) respectively. For HDP (PE and GH combined), the adjusted odds ratio when SDB was present in early pregnancy did not reach statistical significance, but in midpregnancy this was significant (adjusted OR $1.73,95 \% \mathrm{Cl}[1.19-2.52])$. Statistically significant linear trends were also observed between increasing $\mathrm{AHI}$ and the rate of all hypertensive disorders in adjusted analysis $(\mathrm{p}<.001)$, and adjusting for BMI did not alter the direction, magnitude or statistical significance of these effects. This study could be considered limited by the use of abbreviating SDB monitoring, assumingly employed due to the very large sample size. Regardless, this is likely the most robust study to date with good control of confounders. Although the majority of SDB cases identified were mild, the authors conclude that the data demonstrates that even modest elevations of $\mathrm{AHI}$ in pregnancy are associated with increased risk of developing hypertensive disorders. ${ }^{123}$

Whilst the majority of studies have focused on a positive association between hypertensive disease during pregnancy and SDB, it should be noted that a small number have not confirmed this. Pien et al. ${ }^{7}$ performed PSG on 105 pregnant women in the first and third trimester. Twenty of these women developed a hypertensive disorder, however there was no association between degree of SDB at either point of pregnancy and $\mathrm{GH}$ or $\mathrm{PE}$, or with arterial $\mathrm{BP}$ measured in the third trimester. A similarly designed study by Facco et al. ${ }^{263}$ objectively assessed SDB with the Watch-PAT100 in 182 high risk pregnant women between 6-20 weeks gestation and again in the third trimester. Even amongst this vulnerable sample, no relationship was demonstrated between SDB exposure in early or late pregnancy and PE. Both of these studies have strong sample sizes considering objective sleep monitoring was employed, however the authors acknowledge that their studies likely lacked adequate statistical power given the relatively few participants with moderate or severe OSA in the third trimester $(n=5 \%$ and $7 \%$, respectively).

Until recently, most studies have concluded that a relationship between SDB and hypertensive disorders exists, despite closer examination revealing poor adjustment or control of obesity, 8,257,259,260 limited sleep monitoring techniques ${ }^{124,259,263}$ or non-significant results not highlighted in the study conclusions. ${ }^{8,259,260}$ In particular, given that obesity is a well-recognised risk factor for both SDB, ${ }^{264,265}$ and a risk factor for both increased $\mathrm{BP}$ and $\mathrm{PE},{ }^{266,267}$ any assessment of the relationship between SDB and PE needs to carefully consider the effect of BMI. Before any strong conclusion can be drawn, 
studies with larger numbers, gold standard sleep monitoring and careful BMI-matching need to be conducted. Furthermore, assuming SDB promotes hypertension in pregnancy, it is yet to be determined what degree of SDB is clinically relevant and what indices are most predictive of adverse maternal and fetal outcomes.

\subsubsection{Potential Mechanisms of Sleep-Disordered Breathing and Hypertensive Disorders of Pregnancy}

Only a few studies have investigated the physiological mechanisms of how SDB may impact upon the development of hypertensive disorders in pregnancy. An early study in this field found that women with a normotensive pregnancy who snored and experienced minor $\mathrm{SpO}_{2}$ drops during sleep presented the largest increase in BP between the $6^{\text {th }}$ week and $6^{\text {th }}$ month prenatal visits, despite remaining below the pathological range. ${ }^{120}$ Sullivan et al..$^{268}$ similarly found that women who snored for more than $20 \%$ of the night during the third trimester of pregnancy had significantly higher BP, and a larger rise in BP over the duration of pregnancy.

An innovative study performed by Edwards and colleagues ${ }^{269}$ performed full PSG with concurrent continuous beat-to-beat BP recording via finger photoplethysmography, on ten pregnant women with SDB and ten pregnant women with PE and coexisting SDB. Despite similar severity of SDB, there was a marked augmentation of the hemodynamic response to obstructive respiratory events during sleep in women with PE compared with normotensive control patients. The systolic and diastolic BP increases coinciding with apnoea were significantly enhanced in preeclamptic patients compared with control SDB patients ( $21 \pm 2 / 12 \pm 1 \mathrm{mmHg}$ and $38 \pm 5 / 25 \pm 4 \mathrm{mmHg}$ above baseline respectively, $p=.005 / .005)$. The data suggest that the augmented pressor responses in preeclamptic women occur as a result of maternal endothelial damage induced by the evolution of preeclamptic disease.

Edwards et al..$^{270}$ hypothesised that snoring and upper airway obstruction, as well as overt respiratory events, contributes to nocturnal rises in BP in PE. By controlling sleep-induced upper airway flow limitation with CPAP treatment, they were able to show a marked reduction in nocturnal BP compared with the initial study night without treatment. It should be noted that none of these participants actually had significant SDB, rather airflow limitation was diagnosed using a non-validated technique. A followup study used the same methodology of full PSG with beat-to-beat BP recording to measure hemodynamic parameters in women with severe PE compared with normotensive pregnant women. ${ }^{169}$ Sleep induced marked decrements in heart rate, stroke volume, and cardiac output in preeclamptic women with elevation in total peripheral resistance. When preeclamptic subjects were treated with CPAP, reductions in cardiac output were minimised, while increments in total peripheral resistance were also reduced. This further strengthened their hypothesis that milder degrees of upper airway obstruction play a role in altered hemodynamic parameters during sleep in PE.

A study by Yinon et al. ${ }^{184}$ measured SDB with the Watch-PAT100 and evaluated endothelial function with the reactive hyperaemia test (using Endo-PAT200) in females with PE and normotensive females. 
Preeclamptic patients had a significantly higher RDI than controls, but also had a lower endothelial function index, with endothelial function index tending to weakly correlate with RDI. The authors speculate that respiratory disturbances contribute to the functional abnormality of the blood vessels seen in preeclamptic women.

The gold standard treatment for SDB is CPAP, which is a safe and effective treatment during pregnancy. ${ }^{271}$ Only a handful of studies have trialled CPAP as a means of improving pregnancy outcomes, with no published clinical trials to date. One of the first studies was conducted by Guilleminault et al. ${ }^{272}$ and followed 12 women with PE risk factors who underwent CPAP therapy from early pregnancy. These women did not have SDB or oxygen desaturations, however it was found that application of CPAP in pregnant women alleviated flow limitation and snoring but was not sufficient to prevent negative pregnancy outcomes. Rather, the most important factors associated with the worst pregnancy complications were obesity and a prior history of PE. A small randomised study with only 16 participants found a decrease in BP and antihypertensive dose in a small sample of chronic hypertensive pregnant women treated with CPAP longitudinally. ${ }^{273}$ Again these participants exhibited chronic snoring only rather than actual SDB.

Reid et al. ${ }^{274}$ compared SDB treatment methods by assigned 11 women with GH to auto-titrating CPAP verses 13 women with $\mathrm{GH}$ to a mandibular advancement device. Most women demonstrated prolonged periods of inspiratory flow limitation during sleep, with $38 \%$ of the participants demonstrating an RDI $\geq$ 5. CPAP was more effective at treating SDB compared to the mandibular advancement device, however first morning BP and inflammatory markers were not improved with either therapy. It should be noted this was a single night intervention only.

\subsection{The Impact of Sleep-Disordered Breathing on other Maternal Outcomes}

Aside from links to hypertensive disorders, SDB has been associated with other adverse maternal outcomes. A number of studies have related self-reported snoring to a 1.86 to 3.4 fold increased risk of gestational diabetes mellitus (GDM), 9,275,276 however one study of over 1700 pregnant women found no independent association. ${ }^{104}$ Studies relating objective measurement of SDB to GDM have had mixed results. SDB severity in early pregnancy has been associated with the risk of developing GDM with an adjusted OR for moderate/severe SDB of 3.6,263 whereas in another study SDB symptoms were associated with hyperglycaemia but the $\mathrm{AHI}$ in the first and third trimester of pregnancy was not. ${ }^{277}$ Bisson et al. ${ }^{278}$ found no differences in multiple measures of SDB including $\mathrm{AHI}$ and flow limitation across 26 cases of GDM pregnancy and controls matched for gestation, BMI and age, and Pien et al. ${ }^{7}$ also found that in over 100 pregnant women who underwent PSG, SDB was not associated with GDM. Both women reporting symptoms of SDB and those with confirmed SDB were more likely to have a caesarean delivery compared to those without symptoms or a diagnosis. $9,124,262,279,280$ 


\subsection{The Impact of Sleep-Disordered Breathing on Fetal Outcomes}

In addition to the maternal effects, the combination of intermittent hypoxia, sympathetic activation, inflammation and endothelial dysfunction as a result of SDB may contribute to poor placentation and impaired fetal growth, via the mechanisms described earlier.

Studies of self-reported SDB symptoms have failed to clarify any relationship with fetal wellbeing. Some research has suggested that self-reported snoring is related to FGR, low birthweight and decreased Apgar scores at birth.6,281 However, a number of symptom-based studies have not supported this relationship, finding no difference in fetal outcomes between snorers and non-snorers. ${ }^{102,253-255,282,283}$ Alternately, a positive relationship has been demonstrated between endorsement of SDB symptoms and increased infant birthweight. ${ }^{253,254,279}$

Data on the fetal sequelae of objectively-measured SDB during pregnancy are sparse and inconclusive. Three studies to date have simultaneously monitored fetal heart rate during PSG as a means of identifying episodes of fetal distress in response to apnoea and subsequent hypoxia during maternal sleep. In four pregnant women with SDB, fetal heart rate decelerations were reported in three during maternal oxygen desaturations. ${ }^{240}$ In contrast, among 20 women with SDB during pregnancy, none of the apnoea episodes monitored were associated with any fetal heart rate trace abnormality. ${ }^{107}$ Similarly, Fung et al. ${ }^{242}$ showed that despite significant apnoeas with oxygen desaturation below $80 \%$ in some women, respiratory events were largely unaccompanied by acute fetal heart rate responses in a sample of 51 women.

Retrospective analysis of 57 female patients with confirmed SDB found that they were at increased risk of pre-term birth and NICU admission compared to obese and normal weight controls, but not small for gestation age infants. ${ }^{262,280}$ Another large retrospective study of 791 women with SDB identified via the Taiwanese National Health Insurance Research Dataset found that SDB was a risk factor for low birthweight, preterm birth, and low Apgar scores at 5 minutes. ${ }^{262,280}$

Prospectively performed studies have yielded mixed results. On the one hand, a diagnosis of SDB amongst a sample of 175 obese pregnant women has been associated with more NICU admission for infants, but no difference in pre-term birth or birthweight. ${ }^{124}$ Conversely, a study by Fung et al. ${ }^{242}$ found that SDB was associated with impaired fetal growth in the third trimester rather than small for gestational age infants, and no increase in NICU admission. Two longitudinal studies with over 100 participants each used full PSG to objectively measure SDB in the first and third trimester of pregnancy. ${ }^{7,263}$ Both found no associations between SDB variables and pre-term delivery, low birthweight and small for gestational age infants. As mentioned earlier, both of these studies had strong methodologies, however relatively few participants with moderate to severe SDB limits their findings to those with mild SDB.

One study has investigated the effect of treating SDB in PE on fetal wellbeing. Blyton and colleagues 284 used overnight PSG with concurrent fetal movement recording to show that PE was associated with 
reduced total fetal movements overnight compared to a control group. However, treatment of SDB with CPAP increased the number of nocturnal fetal movements significantly.

The current prospective studies using objective measurements of SDB are lacking consistency in their results. Also, most studies have only looked at the fetal outcomes at birth, rather than the impact SDB may have on the fetus while still in utero. There is a clear need for further studies to clarify the acute and chronic impacts of SDB on fetal wellbeing.

\subsection{Importance of the Study}

PE is a hypertensive disorder that affects as many as $10 \%$ of pregnancies, with $10 \%$ of these cases so severe that it represents a serious threat to the life of the baby and the mother. ${ }^{285}$ Despite advances in perinatal care, the incidence of PE has not lessened. ${ }^{286,287}$ Therapeutic options remain limited, with the mainstay of management remaining timely delivery. The various pathological mechanisms that lead to PE are not completely understood and research has not resulted in a reliable prediction method for this disorder. ${ }^{288,289} \mathrm{GH}$ is the main component of $\mathrm{PE}$ and can often progress on to $\mathrm{PE}$, particularly if severe or early onset, and must be monitored carefully.

Outside of pregnancy, SDB confers a 3-fold increase in risk of hypertension, independent of other risk factors ${ }^{126,290}$ and treatment of SDB lowers BP.291 PE and SDB share similar biological pathways, making it plausible that SDB could be a contributor to HDP. Symptoms of SDB are commonly reported in GH and $\mathrm{PE}$, however to date most studies investigating a relationship between SDB and HDP have not yet been convincing with a number of shortcomings. The impact of SDB on fetal outcomes is even less clear, with a relatively small number of studies and conflicting results.

This study will be the first to assess the prevalence of SDB in HDP compared to BMI-matched normotensive controls, therefore ascertaining the unique relationship between SDB and hypertension over and above that related to obesity. This study will also focus on the development of hypertensive disease during pregnancy rather than simply the presence versus absence of disease. In terms of fetal outcomes, we will objectively measure SDB with concurrent CTG and use ultrasound to follow fetal growth trajectory in the third trimester in an attempt to clarify the discrepancies in the literature, therefore assessing acute and chronic fetal responses to obstructed airflow and resulting hypoxaemia. This will enable us to firmly establish whether SDB is related to hypertensive diseases and poor fetal outcomes in pregnancy. If SDB has a negative maternal and/or fetal impact in women with GH or PE, this opens a new treatment option given that effective therapy for SDB is already available in the form of CPAP. This project could provide the basis for a therapeutic trial of CPAP in women with GH or PE and coexisting SDB, with the aim to safely prolong gestation and improve perinatal outcomes.

As a related aside, this study enables the opportunity to be the first to assess PSG-measured PLMS in pregnancy. The related disorder of RLS affects pregnant women two-to-three-fold compared to the general population, and $80 \%$ of RLS patients reportedly experience the stereotyped movements during 
sleep known as PLMS. Despite this, no studies have investigated the prevalence of PLMS during pregnancy and the possible significance of this condition.

\subsection{Aims and Hypotheses}

The primary aim of this study is to investigate the prevalence of SDB in HDP. The primary hypothesis is that pregnant women with HDP will have a higher rate of SDB compared to BMI- and gestationmatched normotensive pregnant women.

The secondary aim of this study is to investigate the acute and chronic effects of SDB on maternal and fetal health. We hypothesise that SDB in addition to HDP is associated with more malignant hypertensive disease, in terms of earlier gestation at diagnosis and reduced prolongation of pregnancy, earlier gestation at delivery, poorer BP control, increased anti-hypertensive requirements and worsening biochemical and angiogenic markers of disease. In terms of fetal health, we hypothesise that SDB in addition to HDP is associated with acute pathological fetal heart rate responses owing to intermittent maternal hypoxia overnight, and associated with chronic effects including impaired fetal growth, alterations in fetal growth hormones on cord blood and worse indicators of fetal wellbeing at birth. Lastly, this study also aims to assess the prevalence of RLS and PLMS during both hypertensive and normotensive pregnancy, and the relationship of these disorders to nocturnal BP, iron and folate levels. 
Chapter 2 - Method

Sleep-Disordered Breathing in Hypertensive Disorders of Pregnancy:

Impact on Maternal and Fetal Outcomes 


\section{Preface - Chapter 2}

The study that was performed for this thesis involved one large research project wherein each participant was involved in all investigational measures and procedures. For this reason, the general method chapter describes the study protocol in full. Each chapter that follows includes the methodology relevant to the results being presented in that chapter only. In the case of published manuscripts, the relevant method in full was required and hence some repetition is unavoidable. Within the unpublished chapters any relevant methodological information has been re-presented as a recap for the reader but has been condensed where appropriate.

For clarity, the terms PE and GH are used when referring to these groups of cases individually, whereas HDP includes participants with both of these hypertensive conditions. 


\subsection{Study Participants}

This was a single-centre prospective case-control study conducted between October 2012 and October 2015, comparing women with the diagnosis of HDP to healthy women with uncomplicated pregnancies. The Human Research Ethics Committees at Austin Health, Mercy Hospital for Women and University of Melbourne approved the study and informed consent was obtained from all participants (Appendix $A$ - Participant Information and Consent Form).

Cases included women with a singleton pregnancy diagnosed with GH, PE or chronic hypertension, not requiring immediate delivery between 26 and 37 weeks gestation. Women with GH or PE were recruited from the Pregnancy Day Assessment Centre or were inpatients at Mercy Hospital for Women. Hypertension in pregnancy was defined as systolic BP greater than or equal to $140 \mathrm{mmHg}$ and/or diastolic BP greater than or equal to $90 \mathrm{mmHg}$, confirmed by a minimum of two readings over several hours. GH was defined as the new onset of hypertension after 20 weeks of gestation. ${ }^{134}$ We used the most recent International Society for the Study of Hypertension in Pregnancy (ISSHP) definition of PE, as per Table 2.1.134 Women with chronic hypertension who developed superimposed PE (diagnosed when one or more systemic features of PE occur after 20 weeks gestation in addition to pre-existing hypertension) were also eligible to participate as a PE case.

Table 2.1134 The Revised ISSHP Definition of Preeclampsia (2014) is

Hypertension developing after 20 weeks gestation and the coexistence of one or more of the following new onset conditions:

1) Proteinuria (spot urine protein/creatinine $\geq 30 \mathrm{mg} / \mathrm{mmol}$ or $\geq 300 \mathrm{mg} /$ day)

2) Other maternal organ dysfunction

a) Renal insufficiency (creatinine $\geq 90 \mathrm{umol} / \mathrm{L}$ - ref range $44-80 \mathrm{umol} / \mathrm{L}$ )

b) Liver involvement (elevated transaminases (ALT ref range $<33 \mathrm{U} / \mathrm{L}$ ) and/or severe right upper quadrant or epigastric pain)

c) Neurological complications (examples include eclampsia, altered mental status, blindness, stroke, or more common hyperreflexia when accompanied by clonus, severe headaches when accompanied by hyperreflexia, persistent visual scotomata)

d) Haematological complications (thrombocytopenia (platelet count ref range 150,000400,000/uL), DIC, haemolysis)

3) Uteroplacental dysfunction

a) Fetal growth restriction

Note. DIC = Disseminated Intravascular Coagulation. Normal values (ref range) added to table.

Women with chronic hypertension were recruited from either the antenatal outpatient clinic or the obstetric medicine clinic. Chronic hypertension in pregnancy includes essential hypertension (without a known cause) as well as secondary hypertension, and is confirmed before pregnancy or before 20 
completed weeks of gestation. Important secondary causes of chronic hypertension in pregnancy include chronic kidney disease, systemic disease with renal involvement, and endocrine disorders. ${ }^{132}$

Control participants were normotensive pregnant women one-to-one matched by BMI to each of the cases (within $\pm 4 \mathrm{~kg} / \mathrm{m}^{2}$, measured at the first antenatal appointment), and were recruited from the antenatal outpatient clinic at the Mercy Hospital for Women. Control participants had otherwise uncomplicated pregnancies and were confirmed to have an appropriately-grown fetus (EFW $>10^{\text {th }}$ centile with normal fetoplacental Doppler studies) prior to inclusion. All potentially eligible women were screened by medical record review. Exclusion criteria included $<18$ years of age, multiple gestation, fetal abnormality or other maternal/fetal condition likely to mandate early or imminent delivery, and previous diagnosis of a sleep disorder. Imminent delivery was also an exclusion, as such hypertensive women past 38 weeks of gestation were typically too close to induction or delivery and not approached for recruitment.

\section{$2.2 \quad$ Investigational Measures}

\subsubsection{Maternal Measures}

\subsubsection{Polysomnography}

Attended overnight PSG was conducted in the Austin Health sleep laboratory using the Compumedics E series (Abbotsford, Victoria, Australia), or unattended in the participant's home with the Somté (Compumedics) portable sleep-monitoring device. Participants who were inpatients were also studied using the portable device. Portable sleep monitoring systems are commonly used in clinical settings, and have been shown to have a high level of agreement with standard laboratory-based systems. ${ }^{292}$ 294 Signals recorded both in-laboratory and portably included electroencephalogram (EEG; with recommended derivations of $\mathrm{F} 4, \mathrm{C} 4$ and $\mathrm{O} 2$ referenced to $\mathrm{M} 1^{38}$ ), electrooculogram (EOG), electromyogram (EMG), electrocardiogram (ECG), arterial oxygen saturation, thoracic and abdominal respiratory effort via inductance plethysmography, nasal airflow measured via nasal cannula, oronasal thermistor, leg movements, snoring and body position. After sensors were placed and calibrated, signal quality and impedances were checked. Recordings were sleep-staged and respiratory-scored by a single experienced sleep technologist, who participates in internal and external quality assurance programs, who was blinded to maternal hypertensive status. As per the American Academy of Sleep Medicine (AASM) criteria ${ }^{295}$, respiratory events were categorised as apnoeas (a decrease in airflow of $\geq 90 \%$ from baseline for $\geq 10 \mathrm{sec}$ ); hypopnoeas (decrease in airflow $\geq 30 \%$ from baseline for $\geq 10 \mathrm{sec}$ and followed by either an oxygen desaturation of $\geq 3 \%$ from the pre-event baseline or a EEG cortical arousal); and respiratory event related arousals (RERAs; a sequence of breaths lasting $\geq 10 \mathrm{sec}$ characterised by increasing respiratory effort or by flattening of the inspiratory portion of the nasal pressure waveform leading to an arousal from sleep). The number of apnoeas and/or hypopnoeas per hour of sleep was calculated as the apnoea-hypopnoea index (AHI). The addition of RERAs per hour 
to the $\mathrm{AHI}$ was expressed as the respiratory disturbance index (RDI). The ODI $\geq 3 \%$ was defined as the number of arterial oxygen desaturations of $\geq 3 \%$ from baseline, per hour of sleep. ${ }^{295}$

All participants diagnosed with SDB at an RDI $\geq 5$ were offered clinical follow up with a sleep physician, whereas those diagnosed at an RDI $\geq 15$ or with clinically significant symptoms (i.e. falling asleep while driving) were given urgent follow up within 2 weeks of the PSG.

Leg movements classified as PLMS were visually scored as per the AASM criteria, ${ }^{38}$ and the PLMS index (PLMSI) was calculated as the number of PLMS per hour of sleep. A leg movement was defined by duration of between 0.5 to 10 seconds with a minimum amplitude of $8 \mathrm{uV}$-increase in EMG voltage above resting EMG. A PLM series required a minimum number of 4 consecutive leg movements, with an inter-movement interval of 5-90 seconds. Leg movements on 2 different legs separated by less than 5 seconds were counted as a single leg movement. A leg movement was not scored if it occurred during a period from $0.5 \mathrm{~s}$ preceding or following an apnoea, hypopnoea or RERA. ${ }^{38}$

\subsubsection{Overnight Blood Pressure Measurement}

Immediately prior to lights out for the PSG and upon awakening, BP was measured from the upper nondominant arm at the brachial artery with an automated device (OMRON Standard BP Monitor HEM7203), via an appropriately sized cuff with the participant in a sitting position. Each measurement was taken twice with the average being used for analysis, and the same arm was used in the evening and morning. Participants undergoing a portable PSG at home were given the BP monitor and instructed on how to perform and record measurements.

\subsubsection{Blood Sampling - Maternal}

A venous blood sample was taken from each participant near the time of the sleep study and again when admitted for delivery. Each sample was assayed for Endothelin-1 (ET-1), sFIt-1, sEng and PIGF. Details for how the blood samples were assayed are given in Chapter 4 - Section 4.2.2.3 page 89.

An additional venous blood sample was taken during the third trimester for the women included in Chapter 6, and was assayed for Iron Studies (iron, transferrin, saturation, and ferritin), red blood cell (RBC) folate, haematocrit and haemoglobin.

\subsubsection{Prediction of Sleep-Disordered Breathing Questionnaires}

The Berlin Questionnaire ${ }^{110}$ (Appendix B) assesses pre-test probability of OSA. The questionnaire contains five questions concerning snoring, three questions addressing daytime sleepiness, and one question concerning presence of high BP and/or increased current BMI. Predetermination of high risk and lower risk for sleep apnoea was based on responses in three categories. In category 1, high risk was defined as persistent symptoms ( $>3$ to 4 times/week) in two or more questions about their snoring. In category 2, high risk was defined as persistent (> 3 to 4 times/week) waketime sleepiness, drowsy 
driving or both. In category 3, high risk was defined as a history of high BP and/or a BMI more than 30 $\mathrm{kg} / \mathrm{m}^{2}$. As per the questionnaire guidelines, to be considered as high risk for OSA a participant had to qualify as high risk for at least two categories. Those with positive answers in at least two categories which were not persistent, or who qualified in only one category were considered low risk for OSA.

Multivariate Apnea Risk (MAP) Index ${ }^{112}$ (Appendix C) is based on a model developed for predicting the diagnosis of OSA. The full version includes 16 self-report symptoms from four domains associated with sleep disorders - SDB, difficulty sleeping including an item pertaining to RLS/PLMS, EDS and narcolepsy-like symptoms. Frequency of each of the symptoms over the past month was scored as follows $-(0)=$ never, $(1)=$ less than once a week, $(2)=1-2$ times per week, $(3)=3-4$ times per week, and $(4)=5-7$ times per week

Restless Legs Syndrome (RLS) questionnaire (Appendix D) - was derived from the four essential diagnostic criteria for RLS. ${ }^{296}$ All four of the following criteria were required to make the diagnosis of RLS - i) an urge to move the legs, usually accompanied or caused by uncomfortable and unpleasant sensations in the legs; ii) the urge to move or unpleasant sensations begin or worsen during periods of rest or inactivity such as lying or sitting; iii) the urge to move or unpleasant sensations are partially or totally relieved by movement, such as walking or stretching, at least as long as the activity continues; and iv) the urge to move or unpleasant sensations are worse in the evening or night than during the day or only occur in the evening or night. Questions regarding the frequency of these symptoms and whether the symptoms were present prior to the current pregnancy were included.

\subsubsection{Medical Records - Maternal/Obstetric Outcomes}

Baseline information including maternal age, ethnicity, parity, BMI recorded at the first antenatal appointment and estimated due date of current pregnancy, as well as relevant comorbidities such as diagnosis of $\mathrm{GDM}^{297}$ were recorded at recruitment. Following delivery, medical records were reviewed regarding diagnosis and severity of hypertensive disease, including gestation at diagnosis of GH or PE, days between diagnosis and delivery of the fetus, use of anti-hypertensive medication, and highest BPs recorded antenatally, postnatally and during the intrapartum period for those who laboured. BP recorded at each antenatal appointment was also recorded. Antenatal appointments were typically every four weeks during the second trimester increasing to every 2 weeks during the third trimester, however hypertensive participants were usually seen more frequently as clinically indicated. Participants diagnosed with or suspected of a hypertensive disorder were routinely sent to the Pregnancy Day Assessment Centre (ranging from three times per week to sporadically as determined by the treating physician) for four-hour BP monitoring and blood sampling for indicators of PE severity - these pathology measures included quantification of proteinuria, serum liver transaminases, uric acid and creatinine, and platelet count.

$\mathrm{GH}$ and PE was considered to be severe if $\mathrm{BP}$ was $\geq 160 \mathrm{mmHg}$ systolic and/or $\geq 110 \mathrm{mmHg}$ diastolic on more than one occasion, and PE was considered as early-onset if diagnosed prior to 34 weeks 
gestation. ${ }^{298}$ Cases of GH or PE were reviewed by a senior obstetrician (Sue Walker) to confirm diagnosis.

\subsubsection{Fetal Measures}

\subsubsection{Fetal Heart Rate Monitoring}

CTG was measured in the form of continuous electronic fetal monitoring (CEFM) using the Monica AN24 fetal heart rate monitor (Monica Healthcare Ltd., Nottingham, UK). The Monica AN24 is a noninvasive monitor requiring the placement of 5 adhesive electrodes onto the maternal abdomen, to monitor fetal ECG, maternal ECG and uterine EMG to measure uterine contractions, and maternal movement (Figure 2.1). Monica is a single set up device that generally requires no adjustment or repositioning of the electrodes, and the wireless design allows the woman to be free to ambulate. The leads are connected to the small portable AN24 unit where the data is stored until the recording is completed - the acquired signals are then converted into a digital format and processed and downloaded to the Monica VS software for review.
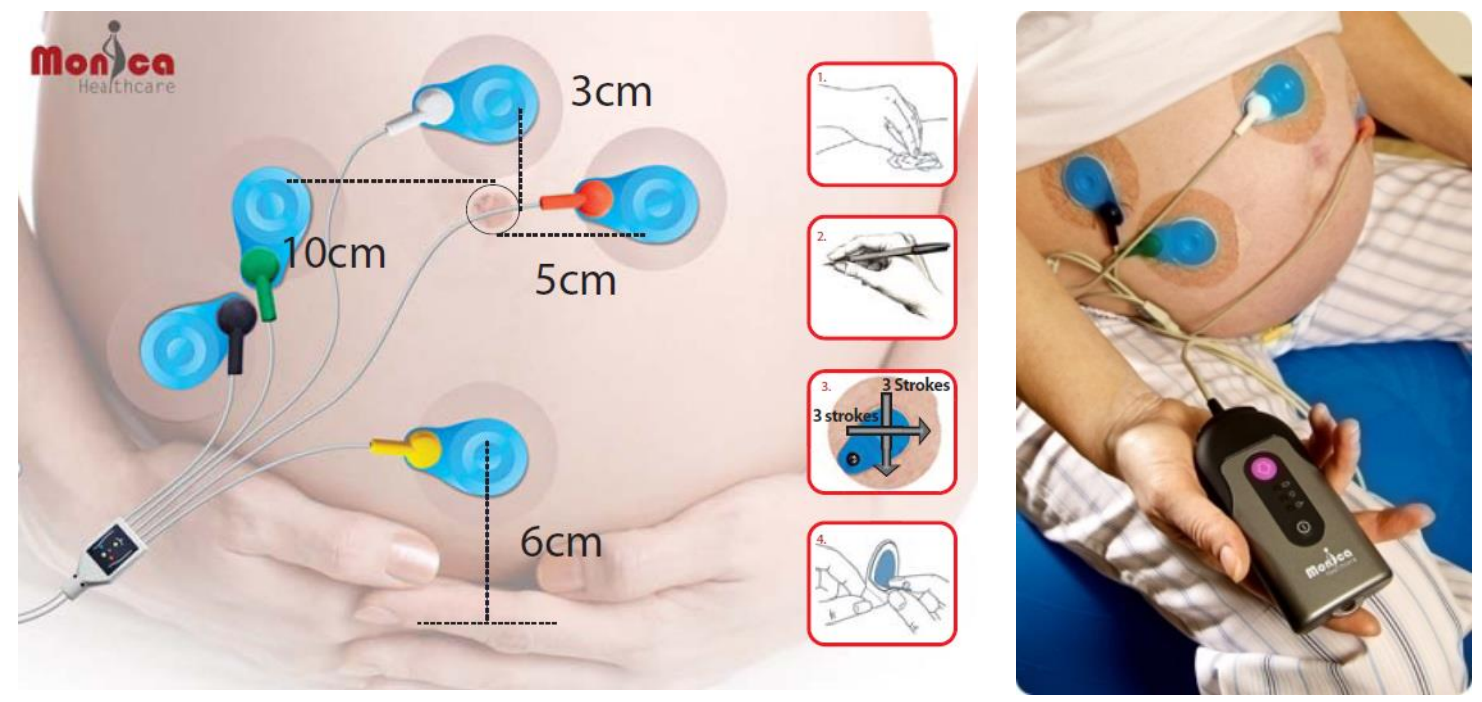

Figure 2.1 Illustration of electrode placement on the maternal abdomen for the Monica AN24 fetal heart rate monitor (Monica Healthcare website).

Fetal heart rate was calculated using the time between the R-waves of consecutive fetal QRS complexes in the ECG signal $(60 / R R$ interval time $=H R)$. Similarly, the time between the R-wave of consecutive maternal QRS complexes in the maternal ECG signal was used to calculate maternal heart rate - this is called the maternal RR interval. The fetal heart rate and maternal heart rate are calculated every $1 / 4$ second using a 'rolling window average'. The amount of averaging is dependent on the ratio of the fetal height to noise and the number of fetal ECG complexes detected, but it is never more than 2 seconds. The fetal heart rate and maternal heart rate data is only made available for viewing when 
the AN24 is confident that this data is accurate, which is based upon the general noise in the recorded data and characteristic shape of the ECG complex.

The Monica VS software program connects wirelessly via Bluetooth to the Monica AN24 for real-time viewing - this was utilised during the initial set up period to ensure adequate signals but was disconnected and not observed during the sleep study. Following skin preparation with an alcohol wipe and abrasive skin tape, the AN24 will check the quality of the electrode connections before commencing a recording.

Each CTG recording was analysed by an obstetrician who was blinded to hypertensive and SDB status for any of four abnormal fetal heart rate monitoring events; i) prolonged bradycardia (slowing of $>15$ beats per min below baseline for $\geq 90 \mathrm{sec}$ and $<5 \mathrm{~min}$ ), ii) recurrent severe variable decelerations (a fall of $>60$ beats per min from previous baseline and of $>60 \mathrm{sec}$ duration and at least 2 per 2 hours), iii) repeated unprovoked decelerations or late decelerations accompanied by tachycardia or loss of variability, and iv) deceleration lasting between 60 to $90 \mathrm{sec}$ and dropping below the baseline by $>15$ beats per min. An example of the CTG output was given in Figures 1.7 and 1.8 in Chapter 1 Introduction.

\subsubsection{Ultrasound}

Ultrasound was performed in the third trimester of pregnancy to monitor fetal growth. These ultrasounds were performed by obstetricians with sub-specialist training in diagnostic ultrasound. The formula used for calculating EFW was based on measurements of head size (head circumference and biparietal diameter), abdominal circumference and femur length. ${ }^{223}$ The ultrasound EFW was customised for maternal height, pre-pregnancy (or if unknown, early pregnancy) weight, ethnicity, parity and fetal sex using the Australian dataset of the GROW software (www.gestation.net). ${ }^{299}$

At the time of delivery, birthweight was also customised as described above, using the GROW software, ${ }^{299}$ so that fetal size at both assessments was adjusted for maternal and fetal characteristics. Fetal growth restriction (FGR) was defined as a customised birthweight $<10^{\text {th }}$ centile for gestational age. Due to variations in the time between gestational age at third trimester ultrasound and delivery, a rate of change in customised centile per day was calculated for each participant, and extrapolated to give a change in customised centile over six weeks (as further discussed in Section 5.2.4 page 135). Evidence of slowed third trimester growth was thereby defined as a projected or actual fall in customised centile of greater than a third (33\%) across six weeks in the third trimester. Impaired fetal growth was defined as either FGR or slowed third trimester growth.

Customisation of EFW and birthweight is superior to population-based weight centiles as it generates an in utero standard, which is then individualised for each fetus, to adjust for relevant maternal characteristics which may affect fetal growth. ${ }^{242}$ The use of customised EFW and birthweight to develop individual intrauterine growth curves appear to be better than population curves at identifying small 
fetuses at risk of increased perinatal complications. ${ }^{225,227}$ Determination of growth velocity with serial measurement and charting along centile curves, rather than a single estimate of abdominal circumference or EFW, has also been shown to be superior in the prediction of FGR and poor perinatal outcome. ${ }^{300}$ The Royal College of Obstetricians and Gynaecologists now recommends the use of customised growth charts and growth velocity in its most recent guideline on the small for gestational age fetus. ${ }^{301}$

\subsubsection{Blood Sampling - Fetal}

Venous cord blood was collected at delivery and analysed for the fetal growth regulators IGF-1, IGF-2, IGFBP-1 and IGFBP-2. Details for cord blood assaying are given in Chapter 5 - Section 5.2.2.4 page 134.

\subsubsection{Medical Records - Perinatal Outcomes}

Following delivery, birthweight was customised as previously described using the GROW software (www.gestation.net), ${ }^{299}$ so that EFW and birthweight were adjusted for gestational age and relevant maternal characteristics.

Medical records were reviewed for the following delivery and fetal outcomes: gestation at delivery, customised birthweight centile, mode of delivery, Apgar scores at $1 \mathrm{~min}$ and $5 \mathrm{~min}$, and admission to the NICU or special care nursery (SCN).

\subsection{Procedures}

A diagram outlining the key procedures involved in this study at their respective gestational ages is shown in Figure 2.2. After consent was obtained, GH and PE participants underwent overnight PSG at their earliest convenience, given that they were usually in the third trimester and time to induction or delivery was a factor. Women with chronic hypertension were typically recruited at an earlier gestation but underwent PSG at a similar gestation to those with GH and PE. Control women were recruited as a one-to-one BMI-match (BMI at the first antenatal appointment) to within $\pm 4 \mathrm{~kg} / \mathrm{m}^{2}$ of a hypertensive case participant, and underwent PSG within \pm 4 weeks of gestational age of that case. 


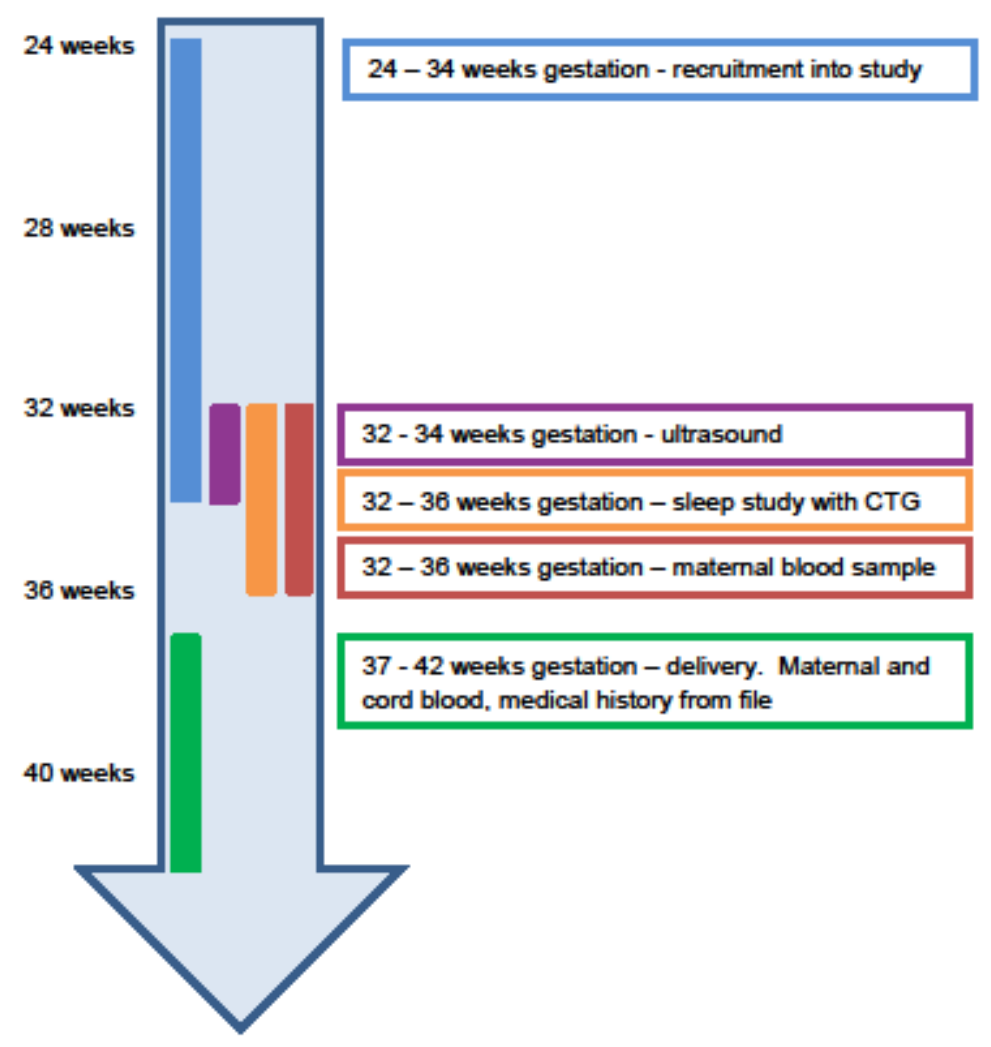

Figure 2.2 Diagram highlighting key procedures involved in the study at planned gestational age of pregnancy. $C T G=$ cardiotocography.

PSG was performed in the sleep laboratory at Austin Health or if preferred, the participant was set-up with the Somte portable sleep monitoring device and allowed to sleep within their own home. Inpatients with $\mathrm{GH}$ or $\mathrm{PE}$ admitted to the antenatal ward were also monitored with the Somte device. The candidate was responsible for all aspects of the in-laboratory studies, and initiated all Somte in-home monitoring and retrieved the equipment the following morning and downloaded the data. At the time of the PSG, height and weight were recorded. Neck circumference was measured at the most prominent part of the thyroid cartilage while the participant was standing. Immediately prior to and immediately following the PSG, BP was measured at the upper arm in the seated position using an automated BP monitor (Omron HEM-7203).

Time-synchronised CTG was performed during the overnight sleep study to enable correlation of any fetal heart rate abnormalities with objectively confirmed respiratory events and/or oxygen desaturations. Approximately one hour prior to PSG set-up, the Monica AN24 fetal heart rate monitor was applied to the maternal abdomen (as per Figure 2.1). Signal quality was confirmed for at least $15 \mathrm{~min}$ in the supine and non-supine position, with electrodes repositioned as required. All fetal heart rate traces were reviewed the following morning by an obstetrician blinded to hypertensive and SDB status, to ensure no abnormalities were present that needed further follow-up and management. 
Around the time of the sleep study (typically within \pm 2 weeks), the participants completed the Berlin Questionnaire, MAP Index and the RLS Questionnaire. Two blood samples were taken by pathology nurses around this time, one for iron studies and the other to measure angiogenic factors.

All women underwent ultrasound for fetal growth between 32-34 weeks gestation (or as soon as recruited if after 34 weeks of gestation). Ultrasound was performed at this time to enable sufficient time between third trimester growth assessment and delivery for any significant change in customised centile and for slowing of fetal growth trajectory to be identified.

At delivery, venous blood from both the mother and the umbilical cord was collected. Participants were given a pack of blood tubes and pathology slips to take with them when admitted to have their baby. If admission for delivery was during business hours a research midwife collected the maternal and cord blood sample after delivery of the baby, otherwise other delivery room/operating theatre staff were relied upon for blood collection. The relevant birth and fetal outcome measures were obtained from medical histories following delivery.

\section{$2.4 \quad$ Statistical Analysis}

\subsubsection{Power Analysis}

At the planning phase of this study, there were very few references indicating the prevalence of objectively-measured SDB in pregnancy, particularly during uncomplicated pregnancy. Preliminary analysis partway through our recruitment identified that women with chronic hypertension were rarely diagnosed with SDB in our sample, and that a very large and unfeasible sample size would be required to show any effect in this group. A decision was made to cease recruitment of participants with chronic hypertension and focus on those with gestationally-based hypertensive disease (GH and PE). By this time, a few more studies had been published regarding prevalence of objectively-measured SDB in pregnancy.

Final sample size was based on the reported prevalence of SDB (RDI $\geq 5)$ in gestational hypertensive disorders being $53 \%^{8}$ compared to $19.7 \%$ in a clinical obstetric population. ${ }^{7}$ A sample size of 64 (32 cases and 32 controls) would be required to provide a power of $80 \%$ (alpha $=0.05$ ) to detect this increased prevalence of SDB in GH/PE. As obesity is a risk factor for both PE and SDB, we suspected that the matched controls would likely be heavier and have a higher prevalence of SDB than previously reported, hence a decision was made to increase the sample size by $25 \%$ ( 40 cases and 40 controls). This updated sample size would provide a power of $80 \%$ (alpha $=0.05$ ) to identify correlations of 0.3 or higher between the severity of SDB and adverse fetal or maternal outcomes (e.g. birthweight centile) within the GH/PE group. 


\subsubsection{Statistical Analysis for each Results Chapter}

The primary aim of this study was to investigate the prevalence of SDB in HDP compared to a control group and is the basis for Chapter 3 . The secondary aims of this study were to investigate the acute and chronic effects of SDB on maternal and fetal health, as described in Chapters 4 and 5. Lastly, Chapter 6 aimed to investigate how frequently PLMS occur in both normotensive and hypertensive pregnancy. In-depth details of statistical analyses are given for the primary and secondary aims of this study in each of the results Chapters 3 through 6. 


\section{Chapter 3 -}

\section{Sleep-Disordered Breathing in Hypertensive Disorders of Pregnancy -}

\section{A BMI-Matched Study.}

Manuscript accepted for publication on $6^{\text {th }}$ December 2017.

Wilson DL, Walker SP, Fung AM, Pell G, O'Donoghue FJ, Barnes M, Howard ME. Sleep-disordered breathing in hypertensive disorders of pregnancy - a BMI-matched study. Journal of Sleep Research, 2018, DOI: $10.1111 / \mathrm{jsr} .12656$.

This is the accepted version of the article, which has been published in final form at Wiley Online Library - http://onlinelibrary.wiley.com and can be found using the article Digital Object Identifier (DOI) $-10.1111 / j s r .12656$. 


\section{Preface - Chapter 3}

The purpose of the first empirical chapter of this thesis was to evaluate the frequency of SDB in women with GH and PE compared to normotensive pregnant women, while controlling for $\mathrm{BMI}$ and gestational age. This chapter was published as a manuscript in the Journal of Sleep Research and hence the style and format follow the specific requirements set out by the journal except where detailed in the main preface (page v). The author accepted version rather than the final published PDF version has been included as per the Copyright Transfer Agreement -

"The Journal of Sleep Research licenses back the following rights to the Contributor in the version of the Contribution that has been peer-reviewed and accepted for publication, but not the final (the "Accepted Version")

- The right to self-archive the Accepted Version in the Contributor's...institutional repository or archive...subject to an embargo period of 12 months for scientific, technical and medical (STM) journals following publication of the Final Published Version.... The Contributor may not update the Accepted Version or replace it with the Final Published Version."

For this project, a total of 102 pregnant women were recruited and underwent PSG. As discussed in the Method - Section 2.4 Statistical Analysis, a decision was made to exclude those with chronic hypertension, leaving a total of 87 participants as used in Chapters 4 and 5 looking at SDB and maternal and fetal outcomes. However, as we were comparing prevalence of SDB in HDP and otherwise healthy normotensive controls for Chapter 3, we had to exclude three participants we studied (1 PE and 2 controls) who had a clinical suspicion of SDB and three control participants who had FGR (with one fetal death in utero). These participants were still recruited to the pregnancy outcome study, as those with suspected SDB could still be used to look at the impact on maternal and fetal outcomes, and those with FGR could be used for the chapter on maternal health. 
Sleep-Disordered Breathing in Hypertensive Disorders of Pregnancy - A BMI-Matched Study

Shortened Title - SDB in HDP with BMI-Matching

Danielle L. Wilson, MSc. ${ }^{1,2,3}$; Susan P. Walker, MBBS, MD, FRANZCOG, DDU, CMFM²,4; Alison M. Fung, MBBS, FRANZCOG, DDU, CMFM²; Gabrielle Pell RN, RM'; Fergal J O'Donoghue, MB, BCh, $\mathrm{PhD}^{1,3}$; Maree Barnes, MBBS ${ }^{1,3}$; Mark E Howard, MBBS, FRACP, GDEB, PhD ${ }^{1,3}$.

${ }^{1}$ Institute for Breathing and Sleep, Austin Health, Heidelberg, Victoria, Australia;

${ }^{2}$ Mercy Perinatal, Mercy Hospital for Women, Heidelberg, Victoria, Australia.

${ }^{3}$ Department of Medicine, University of Melbourne, Parkville, Victoria, Australia.

${ }^{4}$ Department of Obstetrics and Gynaecology, University of Melbourne, Parkville, Victoria, Australia.

Address correspondence to: Danielle L. Wilson, Ground Floor Bowen Centre, Institute for Breathing and Sleep, Austin Health, Heidelberg, Victoria, Australia. Tel: 6139496 3517; Fax: 6139496 5124; email: danielle.wilson@austin.org.au

Mark Howard receives research support from the Resmed Foundation, Philips Respironics and the Cooperative Research Centre (CRC) for Alertness, Safety and Productivity. Maree Barnes receives research support from AirLiquide Healthcare. This article is not related to either relationship. Danielle Wilson, Susan Walker, Alison Fung, Gabrielle Pell and Fergal O'Donoghue declare they have no conflict of interest.

\section{Author Contributorship}

D. Wilson was involved in the study design, data collection, data analysis, interpretation of results and preparation of the manuscript.

S. Walker, M. Howard and F. O'Donoghue were involved in the study design, interpretation of results and preparation of the manuscript.

A. Fung and M. Barnes were involved in the study design and preparation of the manuscript.

G. Pell was involved in the data collection and the preparation of the manuscript.

Word Count - $4998 \quad$ References - 36 


\section{Summary}

Sleep-disordered breathing (SDB) is more common in hypertensive disorders during pregnancy, however most studies have not adequately accounted for the potential confounding impact of obesity. This study evaluated the frequency of SDB in women with gestational hypertension and preeclampsia compared to BMI- and gestation-matched normotensive pregnant women. Women diagnosed with gestational hypertension or preeclampsia underwent polysomnography shortly after diagnosis. Normotensive controls BMI-matched within $\pm 4 \mathrm{~kg} / \mathrm{m}^{2}$ underwent PSG within \pm 4 weeks of gestational age of their matched case. The mean BMI and gestational age at polysomnography were successfully matched for 40 women with gestational hypertension/preeclampsia and 40 controls. The frequency of SDB in the cases was $52.5 \%$ compared to $37.5 \%$ in the control group $(p=.18)$, and the RDI overall did not differ $(p=.20)$. However, more severe SDB was more than twice as common in women with gestational hypertension or preeclampsia ( $35 \%$ vs $15 \%, p=.039$ ). While more than half of women with a hypertensive disorder of pregnancy meet the clinical criteria for SDB, it is also very common in normotensive women of similar BMI. This underscores the importance of adjusting for obesity when exploring the relationship between SDB and hypertension in pregnancy. More severe degrees of SDB are significantly associated with gestational hypertension and preeclampsia, and SDB may plausibly play a role in the pathophysiology of pregnancy hypertension in these women. This suggests that more severe SDB is a potential therapeutic target for reducing the prevalence or severity of hypertensive disorders in pregnancy.

Keywords: Obstructive sleep apnoea, gestational hypertension, preeclampsia, obesity, polysomnography. 


\subsection{Introduction}

Hypertensive disorders of pregnancy (HDP) are the most common and serious medical disorders in pregnancy, affecting 3-8\% of pregnant women and responsible for over 60,000 maternal deaths and far greater numbers of perinatal deaths worldwide every year. ${ }^{2,3}$ Gestational hypertension (GH) is characterised by the new onset of hypertension after 20 weeks gestation, whereas preeclampsia (PE) is diagnosed when $\mathrm{GH}$ is accompanied by multisystem organ dysfunction;134 most typically renal involvement manifesting as proteinuria. Once established, PE inexorably worsens until delivery. PE can have a range of life-threatening complications including maternal liver damage, renal failure and seizures, with increased risks of pre-term birth and fetal growth restriction. ${ }^{3}$ There is no cure, and the mainstay of management is delivery of the fetus. Although $\mathrm{GH}$ alone is generally associated with a good prognosis, up to a quarter of women with GH will go on to develop PE and therefore close surveillance is required. ${ }^{132}$ Because of the disease burden associated with HDP, contributors that have an effective treatment could have an important impact on the health of women and their offspring.

In the non-pregnant population, sleep-disordered breathing (SDB) confers a 3-fold increase in risk of hypertension independent of other risk factors. ${ }^{126}$ Perimenopausal women with SDB have a higher prevalence of hypertension and antihypertensive medication use, ${ }^{127}$ while snoring and symptoms of SDB are commonly reported during pregnancy. ${ }^{6} \mathrm{SDB}$ during pregnancy appears to be linked to adverse outcomes for the mother and baby ${ }^{49,302}$ and in particular, was significantly associated with GH and PE in a recent large prospective cohort study. ${ }^{123}$ On the other hand, obesity is a risk factor for both increased blood pressure during pregnancy and PE. ${ }^{266}$ It is also a well-recognised risk factor for SDB, ${ }^{264}$ and a determinant of developing SDB in pregnancy. ${ }^{7}$ Thus the relative contribution of SDB and obesity to HDP requires independent evaluation.

Several cross-sectional studies have identified an increased frequency of objectively-measured SDB in HDP, but many have not adjusted for the confounding effect of obesity. For example, Reid et al. ${ }^{8}$ demonstrated that women with $\mathrm{GH}$ with or without proteinuria were at least four times more likely to have SDB compared to women with uncomplicated pregnancies. However, the women with GH had a substantially higher body mass index (BMI) on average $\left(37.4 \mathrm{~kg} / \mathrm{m}^{2}\right.$ vs $\left.28.7 \mathrm{~kg} / \mathrm{m}^{2}, \mathrm{p}=.0001\right)$. Other studies have statistically accounted for BMI in multivariate tests, ${ }^{123,257}$ but no studies to date have used a BMI-matched approach to assess the impact of HDP on SDB prevalence.

This study aims to address this gap in the literature, by exploring the relationship between HDP and SDB (assessed with full polysomnography), using a closely matched design to control for the effect of obesity. We hypothesised that pregnant women with HDP (both GH and PE) will have a higher rate of SDB compared to BMI- and gestation-matched normotensive pregnant women. 


\subsection{Method}

\subsubsection{Study Participants}

This was a single-centre cross-sectional study conducted between October 2012 and October 2015, comparing the prevalence of SDB in late pregnancy among women with a hypertensive disorder of pregnancy to women with uncomplicated pregnancies. The Human Research Ethics Committees at Austin Health, Mercy Hospital for Women and University of Melbourne approved the study and written informed consent was obtained from all participants.

Cases included women with a singleton pregnancy diagnosed with GH or PE not requiring immediate delivery between 26 and 37 weeks gestation and were recruited from the Pregnancy Day Assessment Centre or were inpatients at Mercy Hospital for Women. Hypertension in pregnancy was defined as systolic blood pressure greater than or equal to $140 \mathrm{mmHg}$ and/or diastolic blood pressure greater than or equal to $90 \mathrm{mmHg}$, confirmed by a minimum of two readings over several hours. GH was defined as the new onset of hypertension after 20 weeks of gestation. ${ }^{134}$ We used the most recent International Society for the Study of Hypertension in Pregnancy (ISSHP) definition of PE: new hypertension after 20 weeks gestation and one or more of the following new-onset conditions: i) proteinuria, ii) other maternal organ dysfunction (renal insufficiency, liver involvement or neurological complications), and iii) uteroplacental dysfunction - fetal growth restriction. ${ }^{134}$ Women with chronic hypertension who developed superimposed PE (diagnosed when one or more systemic features of PE occur after 20 weeks gestation in addition to pre-existing hypertension) were also considered eligible cases.

Control participants were normotensive pregnant women one-to-one matched by BMI (within $\pm 4 \mathrm{~kg} / \mathrm{m}^{2}$, measured at the first antenatal appointment) to each of the cases, and were recruited from the antenatal outpatient clinic. Control participants had otherwise uncomplicated pregnancies and were confirmed to have an appropriately grown fetus (estimated fetal weight $>10^{\text {th }}$ centile with normal fetoplacental Doppler studies) prior to inclusion. All potentially eligible women were screened by medical record review. Exclusion criteria included $<18$ years of age, multiple gestation, fetal abnormality or other maternal/fetal condition likely to mandate early or imminent delivery, and previous diagnosis of a sleep disorder.

\subsubsection{Investigational Measures}

\subsubsection{Polysomnography (PSG)}

Attended overnight PSG was conducted in the Austin Health sleep laboratory using the Compumedics E series (Abbotsford, Victoria, Australia), or unattended in the participant's home with the Somte (Compumedics) portable sleep-monitoring device. Participants who were inpatients were also studied using the portable device. Signals recorded both in-laboratory and portably included electroencephalogram (EEG; with recommended derivations of $\mathrm{F} 4, \mathrm{C} 4$ and $\mathrm{O} 2$ referenced to $\mathrm{M} 1$ ), electrooculogram (EOG), electromyogram (EMG), electrocardiogram (ECG), arterial oxygen saturation, 
thoracic and abdominal respiratory effort via inductance plethysmography, nasal airflow measured via nasal cannula, oronasal thermistor, leg movements, snoring and body position. Recordings were sleepstaged and respiratory-scored by a single experienced sleep technologist who was blinded to maternal hypertensive status. As per the AASM criteria, ${ }^{295}$ respiratory events were categorised as apnoeas, hypopnoeas, and respiratory event related arousals (RERAs). The number of apnoeas and/or hypopnoeas per hour of sleep was calculated as the apnoea-hypopnoea index ( $\mathrm{AHI}$ ). The addition of RERAs per hour to the $\mathrm{AHI}$ was expressed as the respiratory disturbance index (RDI). The oxygen desaturation index (ODI $\geq 3 \%$ ) was defined as the number of arterial oxygen desaturations of $\geq 3 \%$ from baseline, per hour of sleep. ${ }^{295}$

All participants diagnosed with SDB at an RDI $\geq 5$ were offered clinical follow up with a sleep physician, whereas those diagnosed at an RDI $\geq 15$ or with clinically significant symptoms (i.e. falling asleep while driving) were given urgent follow up within 2 weeks of the PSG.

\subsubsection{SDB Questionnaire}

The Berlin Questionnaire ${ }^{110}$ (Appendix B) assesses pre-test probability of obstructive sleep apnea (OSA). Predetermination of high risk and low risk for OSA was based on responses in three symptombased categories - snoring, daytime sleepiness, and high blood pressure and/or BMI.

\subsubsection{Procedures}

After consent was obtained, participants with GH and PE underwent overnight PSG at their earliest convenience. Each control participant underwent PSG within \pm 4 weeks of gestational age of their matched case. At the time of the PSG, the Berlin Questionnaire was completed and height and weight were recorded. Neck circumference was measured at the most prominent part of the thyroid cartilage while the participant was standing. A sleep technologist with over 10 years of experience (DW) was responsible for all sleep monitoring and anthropometric measurements.

\subsubsection{Statistical Analysis}

All statistical analyses were performed with SPSS 21.0 (SPSS Inc., Chicago, Illinois). Values are given in means with standard deviations $(M \pm S D)$ or median and interquartile range (Mdn (IQR)) for nonnormally distributed variables. A two-sided $p$ value of less than 0.05 was considered to indicate statistical significance. SDB as the primary outcome was defined as an RDI of $\geq 5$ events per hour. Secondary outcomes included diagnosis of SDB using stricter cut-offs of RDI $\geq 10, \geq 15$ and $\geq 30$, and median RDI.

To test the hypothesis that SDB was more common in women with HDP, the proportion of those with an $\mathrm{RDI} \geq 5,10,15$ and 30 were compared to the control group using the chi-square test. Within-subjects analysis for SDB and sleep variables was conducted using paired sample t-tests for normally-distributed 
continuous variables and Wilcoxon signed-rank tests for non-normally distributed continuous variables. Cross tabulations were done to determine sensitivity and specificity values for the Berlin Questionnaire.

Sample size was based on the previously reported prevalence of SDB (RDI $\geq 5$ ) in gestational hypertensive disorders being $53 \%{ }^{8}$ compared to $19.7 \%$ in a clinical obstetric population. ${ }^{7} \mathrm{~A}$ sample size of 64 (32 cases and 32 controls) would be required to provide a power of $80 \%$ (alpha $=0.05$ ) to detect this increased prevalence of SDB in HDP. As obesity is a risk factor for both PE and SDB, we suspected that the matched controls would likely be heavier and have a higher prevalence of SDB than previously reported, hence a decision was made to increase the sample size by $25 \%$ ( 40 cases and 40 controls).

\subsection{Results}

A total of 81 pregnant women participated (see Consort Diagram, Figure 3.1), with one home sleep study failure in the control group due to signal loss. An in-laboratory sleep study was chosen by 22 participants and a home sleep study chosen by 58 . The severity of SDB was not different between these two groups $(p=0.45)$; the only difference in overall sleep quality was better sleep efficiency for those who slept at home $(81.7 \% \pm 9.8)$ compared to the laboratory $(76.0 \% \pm 10.7, p=.03)$.

Table 3.1 confirms the hypertensive and control groups were carefully matched for age, BMI and gestational age at the time of the sleep study. Average age was $32.9 \pm 4.6$ years and BMI at first antenatal appointment and at the time of the sleep study was $32.6 \mathrm{~kg} / \mathrm{m}^{2} \pm 7.1$ and $36.3 \mathrm{~kg} / \mathrm{m}^{2} \pm 6.6$ respectively, with no differences between cases and controls. Neck circumference was significantly larger for the HDP women compared to their BMI-matched controls. Sleep studies in both groups were performed at approximately 33 weeks gestation. At the time of the sleep study, $23(58 \%)$ of the hypertensive participants were taking anti-hypertensive medication, most commonly Labetalol, Aldomet, or a combination of the two. 


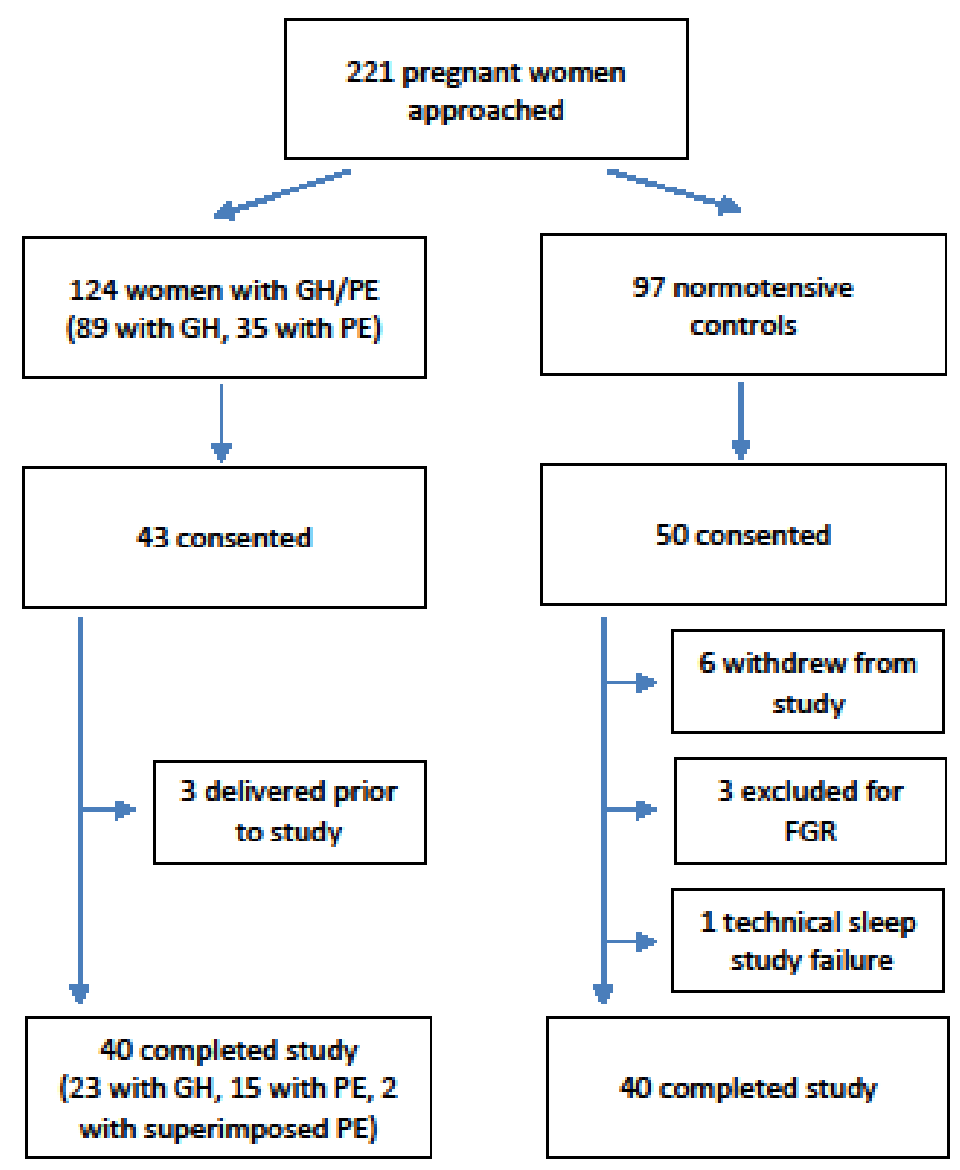

Figure 3.1 Consort diagram. $G H=$ gestational hypertension; $P E=$ preeclampsia; $F G R=$ fetal growth restriction.

Table 3.1 Demographics for Gestational Hypertension and Preeclampsia Cases with Matched Controls

\begin{tabular}{lccc}
\hline $\mathrm{M} \pm \mathrm{SD}$ & $\begin{array}{c}\text { Cases } \\
(\mathrm{n}=40)\end{array}$ & $\begin{array}{c}\text { Controls } \\
(\mathrm{n}=40)\end{array}$ & p value \\
\hline Age (years) & $32.5 \pm 4.7$ & $33.3 \pm 4.4$ & .47 \\
Nulliparous & $26(65.0 \%)$ & $19(47.5 \%)$ & .12 \\
First appointment BMI $\left(\mathrm{kg} / \mathrm{m}^{2}\right)$ & $32.4 \pm 7.3$ & $32.7 \pm 7.0$ & .83 \\
Sleep study BMI $\left(\mathrm{kg} / \mathrm{m}^{2}\right)$ & $36.2 \pm 7.0$ & $36.4 \pm 6.1$ & .90 \\
Neck circumference $(\mathrm{cm})$ & $38.1 \pm 2.0$ & $35.6 \pm 2.0$ & $<.001$ \\
Gestation sleep study (weeks) & $33.5 \pm 3.4$ & $33.1 \pm 2.4$ & .59
\end{tabular}

Note. $\mathrm{BMI}=$ body mass index. 


\subsubsection{Prevalence of SDB}

Using our a priori definition of $\mathrm{RDI} \geq 5,36 / 80$ (45\%) women in the study met the diagnostic criteria for SDB. SDB was more commonly observed among women with HDP (53\%) than the control group (38\%) but this difference was not statistically significant (Table 3.2). An RDI $\geq 10$ was significantly more common among women with $\operatorname{HDP}(35 \%$ compared to $15 \%, \mathrm{p}=0.04)$. A similar trend was seen for moderate SDB $(R D I \geq 15)$ and severe SDB $(R D I \geq 30)$ but due to small numbers, these associations failed to achieve statistical significance (Table 3.2). There were no differences in the prevalence of SDB according to the severity of hypertensive disease (see Figure 3.2).

Table 3.2 RDI Categories for Gestational Hypertension and Preeclampsia Cases with Matched Controls

\begin{tabular}{lccc}
\hline$n(\%)$ & $\begin{array}{c}\text { Cases } \\
(\mathrm{n}=40)\end{array}$ & $\begin{array}{c}\text { Controls } \\
(\mathrm{n}=40)\end{array}$ & p value \\
\hline $\mathrm{RDI} \geq 5$ & $21(52.5 \%)$ & $15(37.5 \%)$ & .18 \\
$\mathrm{RDI} \geq 10$ & $14(35.0 \%)$ & $6(15.0 \%)$ & .039 \\
$\mathrm{RDI} \geq 15$ & $9(22.5 \%)$ & $5(12.5 \%)$ & .24 \\
$\mathrm{RDI} \geq 30$ & $6(15.0 \%)$ & $3(7.5 \%)$ & .29 \\
\hline
\end{tabular}

Note. $\mathrm{RDI}=$ respiratory disturbance index.

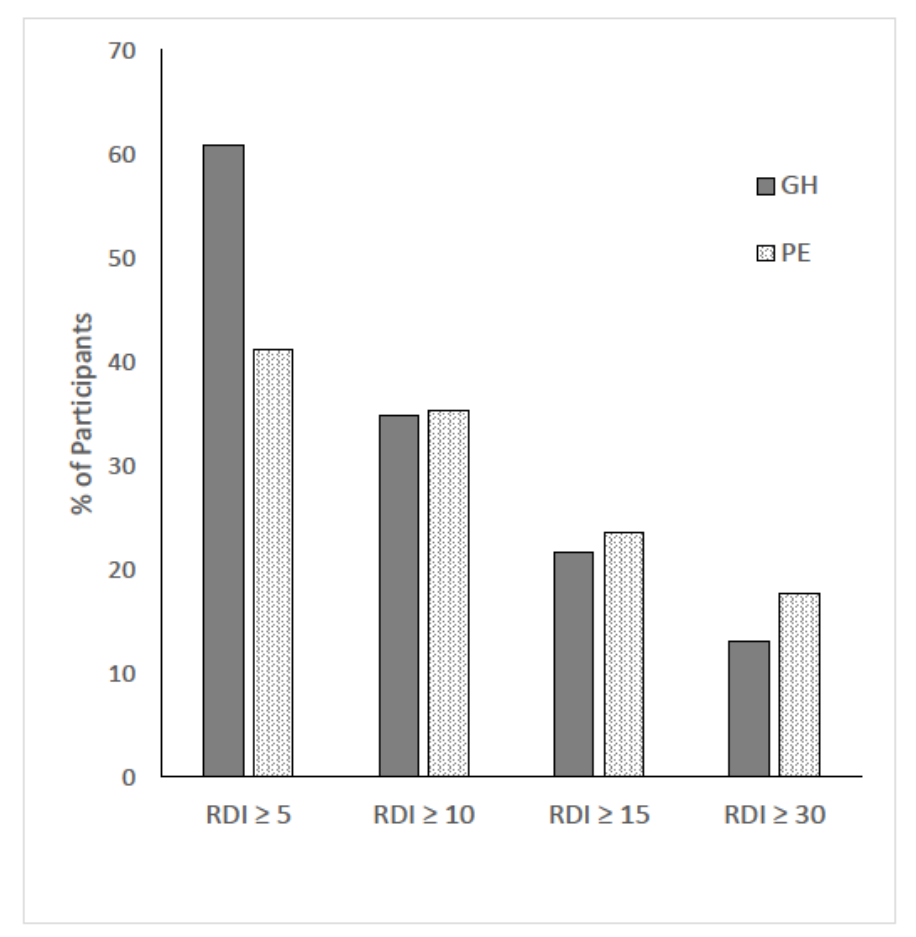

Figure 3.2 Percentage of participants in each Respiratory Disturbance Index (RDI/hr) category for the GH and PE groups. There was no difference in prevalence at $R D I \geq 5(p=.34), \geq 10, \geq 15$ or $\geq 30$ (all $p=1.0$ ). $G H n=23, P E n=17$. 
The median RDI and AHI did not differ between the HDP and control group overall (Table 3.3), however, the HDP group had a significantly higher RDI during non-rapid eye movement (NREM) sleep and supine sleep (Figure 3.3). There were trends toward a higher $\mathrm{ODI} \geq 3 \%$ and a lower $\mathrm{SpO}_{2}$ nadir in the HDP group but this was not statistically significant.

Table 3.3 Indices of SDB for Gestational Hypertension and Preeclampsia Cases with Matched Controls

\begin{tabular}{llll}
\hline $\operatorname{Mdn}(\mathrm{IQR})$ & Cases & Controls & p value \\
& $(n=40)$ & $(n=40)$ &
\end{tabular}

\begin{tabular}{|c|c|c|c|}
\hline RDI & $5.2(2.0,14.8)$ & $4.1(2.3,7.7)$ & .20 \\
\hline $\mathrm{AHI}$ & $4.7(1.6,14.1)$ & $3.9(2.3,7.7)$ & .38 \\
\hline ODI $\geq 3 \%$ overall & $2.1(0.4,6.0)$ & $1.5(0.5,4.4)$ & .06 \\
\hline $\mathrm{SpO}_{2}$ nadir & $90.0(87.3,92.8)$ & $91.0(89.0,92.0)$ & .09 \\
\hline$\%$ TST SpO $2<95 \%$ & $11.3(0.6,36.7)$ & $13.7(1.7,52.4)$ & .39 \\
\hline
\end{tabular}

Note. $\mathrm{RDI}=$ respiratory disturbance index; $\mathrm{AHI}=$ apnea hypopnea index; ODI = oxygen desaturation index, TST $=$ total sleep time.

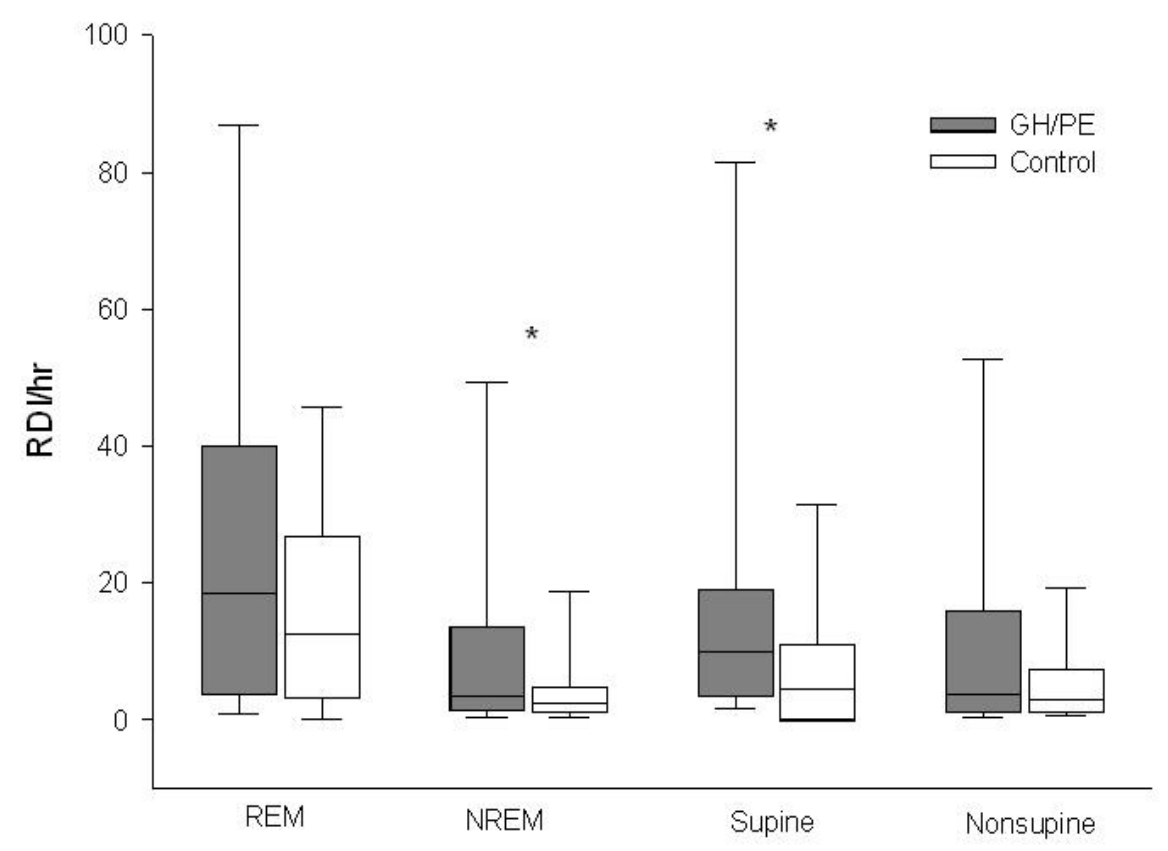

Figure 3.3 Respiratory Disturbance Index (RDI/hr) across different sleep stages and positions for the GH/PE group compared to the control group ( ${ }^{*} p$ value <.05). REM = Rapid Eye Movement sleep; NREM = Non-rapid Eye Movement Sleep. 
Sleep quality was comparable across the HDP group and their matched controls, apart from a lower percentage of REM sleep in the HDP group (Table 3.4).

Table 3.4 Sleep Quality for Gestational Hypertension and Preeclampsia Cases with Matched Controls

\begin{tabular}{lccc}
\hline & $\begin{array}{c}\text { Cases } \\
(\mathrm{n}=40)\end{array}$ & $\begin{array}{c}\text { Controls } \\
(\mathrm{n}=40)\end{array}$ & p value \\
\hline TST $(\mathrm{min})$ & $380.3 \pm 81.2$ & $391.2 \pm 64.7$ & .54 \\
Sleep efficiency \% & $79.5 \pm 10.9$ & $81.0 \pm 9.8$ & .53 \\
$\%$ REM & $13.6 \pm 7.1$ & $16.2 \pm 4.6$ & .03 \\
$\%$ N3 & $32.9 \pm 13.0$ & $31.8 \pm 11.2$ & .66 \\
Sleep latency & $7.8(4.5,15.5)$ & $9.8(4.6,18.0)$ & .30 \\
\%TST supine & $29.1(8.3,41.8)$ & $17.7(0.7,39.7)$ & .49 \\
Arousal Index & $22.5(17.6,32.6)$ & $22.0(16.8,28.1)$ & .59
\end{tabular}

Note. TST = total sleep time; REM = rapid eye movement; N3 = stage N3 sleep. Values given as M \pm SD for normally distributed and Mdn (IQR) for non-normally distributed data.

Within our cohort, neck circumference was a significant predictor of HDP (OR 1.58, 95\% Cl $1.20-$ 2.08), however the trend towards more commonly observed SDB in the HDP group could not be explained by a relationship between neck circumference and $R D I\left(r_{s}=.12, p=.33\right)$. RDI was weakly related to $\mathrm{BMI}$ at the sleep study $\left(r_{s}=.26, p=.03\right.$ ); those with an $\mathrm{RDI} \geq 5$ had a higher BMI than those without SDB in the HDP group $\left(38.4 \mathrm{~kg} / \mathrm{m}^{2} \pm 6.4\right.$ vs $\left.33.8 \pm 7.0, \mathrm{p}=.04\right)$ with a similar trend observed in the control group ( $38.5 \pm 6.7$ vs $35.1 \pm 5.5, p=.09)$.

\subsubsection{Self-Report Data}

The majority of women in both the hypertensive and control group were considered at high risk of SDB (Table 3.5). Significantly more women in the HDP group reported snoring at least three nights per week than their matched controls, and there was a trend towards more frequently witnessed apnoeas.

With the frequency of SDB-related symptoms reported by the participants, the Berlin Risk score overestimated the number of pregnant women with an RDI $\geq 5$ and had poor specificity (sensitivity $=0.81$, specificity $=0.33, \mathrm{PPV}=0.47, \mathrm{NPV}=0.70$ ). Using habitual snoring only as a predictor of $\mathrm{RDI} \geq 5$ did not improve prediction of SDB (sensitivity $=0.60$, specificity $=0.41$, PPV $=0.47, \mathrm{NPV}=0.54$ ). 


\begin{tabular}{lccc}
\hline & Cases & Controls & p value \\
\hline Berlin High Risk & $30 / 37(81.1 \%)$ & $25 / 38(65.8 \%)$ & .19 \\
Habitual Snoring & $22 / 30(73.3 \%)$ & $16 / 34(47.1 \%)$ & .04 \\
Witnessed Apneas & $7 / 36(19.4 \%)$ & $2 / 37(5.4 \%)$ & .09 \\
\hline
\end{tabular}

Note. Missing data due to response of "I don't know".

\subsection{Discussion}

Previous research has suggested a relationship between HDP and SDB, however this study is the first to prospectively address the potential confounding role of obesity using BMI-matching and gold standard PSG to assess SDB. We found SDB to be more common in our control group than in previous studies, 8,260 confirming that $\mathrm{BMI}$ is an important covariate that requires evaluation in future studies exploring the relationship between SDB and HDP. While SDB occurred in more than half of women with HDP, over a third of BMI-matched controls also had SDB. We found no increase in the overall median $\mathrm{RDI}$ and $\mathrm{AHI}$ in the HDP group compared to BMI-matched controls. In a planned secondary analysis, we report a significant independent association between HDP and moderate SDB, suggesting that SDB may still play an important role in the development of hypertension in pregnancy.

That the relationship between HDP and SDB is partly confounded by the presence of obesity is unsurprising, since obesity is a known risk factor for both. SDB may be a mechanism by which obesity and adverse perinatal outcomes are linked, but given the important contribution of obesity to both SDB and HDP, failing to adjust for this co-variate will overestimate the strength of association between SDB and HDP. A dose response relationship has been described for both GH and PE among women with Class 1,2 and 3 obesity, ${ }^{303}$ and in a large Danish population cohort of nearly 71,000 women, the risk of PE increased linearly with BMI among nulliparous women. ${ }^{267}$ Obesity drivers of HDP include increased sympathetic activation, inflammation and oxidative stress. These contribute to placental ischemia, which in turn leads to placental production and release of anti-angiogenic factors such as soluble fms-like tyrosine kinase-1 (sFlt-1) and soluble endoglin (sEng). These circulating factors cause widespread endothelial dysfunction, with the resultant multisystem damage seen in PE. ${ }^{136}$ Obesity is also a strong risk factor for SDB in the non-pregnant ${ }^{264}$ and pregnant population ${ }^{123}$ and in particular, BMI, neck girth and central fat patterning may independently contribute to SDB. ${ }^{304}$ Excess body weight may affect breathing in numerous ways, including changes in upper airway structure or function, reduced chest wall compliance and alterations in the balance between ventilatory drive and load. ${ }^{305}$ Interestingly, within our sample we had six women with a BMI of around $50 \mathrm{~kg} / \mathrm{m}^{2}$, but only two of these women had SDB (one with GH/PE and one control). There are clearly reasons other than obesity- such as changes in hormone levels, respiratory function, and the upper airway and nasal mucosa- which determine whether SDB will be present during pregnancy. Protective factors, such as increased progesterone stimulating ventilatory drive and increasing responsiveness of the upper airway dilator muscle, ${ }^{98}$ may explain why some women with such a high BMI escape SDB. 
Consistent with the observations of others, ${ }^{123,257}$ we confirm a significant association of moderatesevere SDB and HDP. In particular, we found similar frequencies of SDB in HDP across RDI categories to both Reid et al. ${ }^{8}$ and Facco et al. ${ }^{259}$ and like Reid et al. we found no differences in SDB frequency between GH and PE. We found at the level of RDI $\geq 10$ and $R D I \geq 30$ a doubling of prevalence for the $\mathrm{GH} / \mathrm{PE}$ compared to the control group, implying that more severe levels of SDB may contribute to the development of HDP. SDB and PE share similar biological pathways. The recurrent episodes of hypoxia in SDB are known to result in sympathetic activation, oxidative stress and activation of inflammatory pathways that can lead to endothelial dysfunction ${ }^{161}$; a weak negative trend between SDB severity and endothelial function in PE women has been described by Yinon et al. ${ }^{184}$ These are the proposed mechanisms by which SDB results in hypertension and may plausibly contribute to the development of PE. In the same manner, fetal well-being may also be compromised by SDB contributing to placental ischemia. Habitual snoring in pregnancy has been associated with enhanced fetal erythropoiesis, ${ }^{206}$ and women with SDB show significantly increased placental markers of chronic hypoxia and uteroplacental underperfusion. 234

It is possible that the observed high prevalence of SDB identified after being diagnosed with HDP in our study is partly due to physiological changes accompanying HDP. PE is associated with marked fluid retention that affects the upper airway resulting in pharyngolaryngeal edema. ${ }^{302}$ As a result, upper airway size is smaller in PE. ${ }^{306}$ We also found that our GH/PE group had a significantly larger neck circumference than the controls despite almost identical BMI, suggesting that SDB may be exacerbated by the presence of PE-related upper airway oedema. This is partly supported by our novel finding that RDI was higher among hypertensive women during supine sleep. Within our cohort however, we did not find any relationship between neck size and severity of SDB.

Our study along with others has confirmed that subclinical and undiagnosed mild SDB is extremely common among women with higher BMI, irrespective of whether they have HDP. ${ }^{7,124}$ It is not feasible to offer treatment to over a third of all women with an increased BMI, therefore future research needs to determine at what severity threshold SDB should be considered significant enough to intervene. In our study, all women with SDB were offered clinical review with a sleep physician, yet only three of these women attended and were commenced on CPAP. This has been identified in other studies ${ }^{263}$ and suggests that women themselves regard this as relatively low priority and may not commit to what they perceive as onerous treatment. Better engagement may be more likely for women with more severe disease if stronger links with adverse pregnancy outcome are demonstrated.

Strengths of this study were that we used gold standard full PSG to characterise SDB rather than questionnaire data alone, which tends to overestimate the likelihood of SDB. ${ }^{109,307}$ Aligning with many past studies, ${ }^{8,260,306}$ habitual snoring was reported by a substantial proportion of normotensive pregnant women and significantly more GH/PE women. Witnessed apnoeas were also reported by a fifth of GH/PE women. These symptoms along with high BMI and hypertension meant that many women in our cohort were considered as high risk for SDB on the Berlin Questionnaire. Unfortunately, screening 
tools will significantly overestimate the presence of SDB within hypertensive and obese cohorts of pregnant women, which limits their utility in clinical practice.

Use of full PSG also enabled us to look at differences across sleep stages and body positions and detect subtle changes in airflow as well as more discrete events. In particular, we found that SDB in NREM sleep and supine sleep was significantly more severe in HDP compared to normotensive women despite no difference in overall RDI. This is a strength in comparison to studies that have used abbreviated monitoring techniques. ${ }^{122-124,184,259}$ Abbreviated devices may underestimate SDB prevalence due to estimation of sleep time, and absence of EEG monitoring means hypopneas with arousal will be undetected.

Our study utilised domiciliary PSGs as well as in-laboratory. This may be considered a limitation as unattended studies are prone to signal quality problems, however we had a high success rate with only one failure from 58 home studies. Almost three-quarters of our sample chose to have their sleep study at home. Anecdotally, the pregnant women were not keen on spending an extra night in the hospital in an unfamiliar bed, particularly those who were receiving additional antenatal monitoring for clinical reasons. Our results may be useful for future research in this area, demonstrating that women who have a home study may sleep better in terms of sleep efficiency, but measures of SDB are comparable.

A further limitation of our study is that we were underpowered for our primary outcome given the higher than expected prevalence of SDB in the control group. In order to have enough power to obtain a significant difference between frequency of SDB in HDP and BMI-matched controls in our study, we would have needed 169 participants per group. Nevertheless, this is important information to inform the sample size and power of future studies, particularly when matching for the important covariate of BMI.

\subsubsection{Conclusion}

SDB occurs in half of women diagnosed with a gestational hypertensive disorder however it is also common in normotensive women of similar body habitus, with our results suggesting that the previously reported relationship between SDB and hypertension in pregnancy is due at least in part to the confounding effect of obesity. More severe SDB was at least twice as common in women with HDP, suggesting a multifactorial relationship with directionality still needing to be established. The high prevalence of this condition raises questions of a threshold of clinical significance, with further research required to determine at what severity the value of treatment should be tested. 


\subsection{Acknowledgments}

Thank you to the staff at the antenatal clinics at Mercy Hospital for Women and the Sleep Laboratory at Austin Health for their support of this project. In particular, thank you to Pavlina Toman for polysomnogram analysis.

This research was supported by the Austin Medical Research Foundation and the Medical Research Foundation for Women and Babies. Author D. Wilson was supported by a National Health and Medical Research Council (NHMRC) postgraduate scholarship. 
Chapter 4 -

The Impact of Sleep-Disordered Breathing in Pregnancy

on Immediate and Long-Term Maternal Outcomes 


\section{Preface - Chapter 4}

Given the prevalence of SDB in both HDP and normotensive obese pregnant women identified in Chapter 3, this chapter focused on identifying how SDB may influence the course of hypertensive disease during pregnancy and the potential physiological mechanisms underpinning this relationship. This chapter includes control participants with FGR and participants who were excluded from Chapter 3 for clinical suspicion of SDB, as Chapter 4 focuses on the sequalae of SDB on maternal health only. This chapter is currently unpublished however has been written in a style to facilitate submission to an appropriate peer-reviewed journal in due course. 


\subsection{Introduction}

We have demonstrated in Chapter 3 that SDB is common in women with a hypertensive disease of pregnancy, and that more severe levels of SDB occur twice as often compared to BMI-matched normotensive women. Within pregnancy, few experimental studies have attempted to identify the physiological mechanisms underpinning this relationship between SDB and HDP. 169,184,269,270

Among pregnant women with SDB, increased sympathetic activity in response to hypoxaemia and hypercapnia with consequent chemoreflex activation may increase peripheral vascular tone, leading to elevations in systemic arterial BP. ${ }^{169-171}$ Baroreflex and chemoreflex dysfunction in patients with sleep apnoea may, in part, contribute to sustained sympathetic activation and increased BP during the daytime.$^{308-310}$ It stands to reason that pregnant women with SDB should be more prone to hypertension with higher awake and nocturnal BPs, as has been demonstrated in non-pregnant women. ${ }^{127}$

Along with the sympathetic activation and hypertension, SDB also generates inflammation, oxidative stress, and the release of other factors that could contribute to placental dysfunction. Placental dysfunction results in the release of anti-angiogenic proteins and other inflammatory mediators that generate widespread endothelial dysfunction, characteristic of PE. ${ }^{136}$ In particular, the anti-angiogenic factors sFlt-1 and sEng have been implicated in the pathogenesis of many of the maternal features of PE. ${ }^{195}$ SFIt-1 and SEng work as potent signalling inhibitors of pro-angiogenic factors such as PIGF and transforming growth factor- $\beta 1$ (TGF- $\beta 1$ ), which promote proliferation and survival of endothelial cells. ${ }^{136}$

The recurrent episodic hypoxia and reoxygenation of SDB may further worsen placental ischaemia, impacting on the release of these proteins and subsequent damage to the maternal endothelium. Hypoxaemia also triggers the production of ET-1,311,312 a potent long-acting vasoconstrictive peptide with BP-elevating properties. ${ }^{173}$ Recurrent hypoxaemia as a consequence of SDB is accompanied by increases in ET-1 levels ${ }^{313,314}$ which are known to be elevated in PE. ${ }^{315-317}$

GH and PE have different epidemiologic, ${ }^{318}$ pathologic, ${ }^{319}$ pathogenetic ${ }^{320,321}$ and hemodynamic characteristics, ${ }^{322}$ yet the challenge for clinicians is determining whether a patient with hypertension has $\mathrm{GH}$ or whether this is an early manifestation of PE yet to appear. GH involves transient hypertension with usually good outcomes, ${ }^{323}$ whereas PE is accompanied by evidence of end-organ damage and increased risk of adverse pregnancy outcomes. ${ }^{144}$ SDB may thus affect the course of GH and PE differently. For example, among women with GH, SDB may impact on BP control and development of $\mathrm{PE}$, while for women with PE, SDB may impact disease progression and severity. For these reasons, the effect that SDB has on the health of women with GH and PE is considered separately for the majority of the analyses in this chapter. 


\subsubsection{Aims and Hypotheses}

In this study, we aimed to see whether the presence of SDB influenced the course of hypertension in pregnancy. Specifically, we hypothesised that

i) women with PE and SDB have more malignant disease compared to women with PE without SDB, evidenced by -

a.earlier diagnosis of PE and shorter gestation until delivery following PE diagnosis

b.earlier gestation at delivery

c.poorer BP control and heightened nocturnal BP

d.increased anti-hypertensive requirements

e.worsening biochemical and angiogenic markers of disease

ii) women with GH and SDB have more serious disease compared to women with GH without SDB, evidenced by -

a.earlier diagnosis of GH and shorter gestation until delivery following GH diagnosis

b.earlier gestation at delivery

c.development of $\mathrm{PE}$

d.poorer BP control and heightened nocturnal BP

e.increased anti-hypertensive requirements

f.worsening biochemical and angiogenic markers of disease

iii) normotensive control women with SDB are more likely to develop hypertension leading to GH or PE in the remainder of the pregnancy compared to women without SDB, evidenced by-
a. development of $\mathrm{GH}$ or $\mathrm{PE}$
b. poorer BP control and heightened nocturnal BP
c. worsening angiogenic markers of disease

\subsection{Method}

\subsubsection{Study Participants}

As described in Chapter 2 - Method Section 2.1 page 59, this study was initially a prospective casecontrol study. Cases included women with a singleton pregnancy diagnosed with $\mathrm{GH}$ or PE not requiring immediate delivery between 26 and 37 weeks gestation, and control participants were normotensive women one-to-one matched by BMl and gestational age to each of the cases (within $\pm 4 \mathrm{~kg} / \mathrm{m}^{2}$, measured at the first antenatal appointment and within \pm 4 weeks of gestational age), with uncomplicated pregnancies. To evaluate the impact of SDB on maternal outcomes related to $\mathrm{GH}$ and $\mathrm{PE}$, participants were further divided into those with and those without SDB.

Hypertension in pregnancy, and diagnosis of GH and PE were defined as per Chapter 2 - Method Section 2.1 and Table $2.1^{134}$ on page 59. Women with chronic hypertension who developed superimposed PE were also eligible to participate as a PE case. 


\subsubsection{Investigational Measures}

\subsubsection{Polysomnography}

Overnight PSG was conducted either in the Austin Health sleep laboratory using the Compumedics E series (Abbotsford, Victoria, Australia), or in the participant's home with the Somte (Compumedics) portable sleep-monitoring device. Participants who were inpatients at the Mercy Hospital for Women were also studied using the portable Somté device. See Chapter 2 - Method Section 2.2.1.1 page 60 for more detailed information on signals used and sleep and respiratory scoring criteria.

\subsubsection{Overnight Blood Pressure Measurement}

Immediately prior to lights out for the PSG and upon awakening, BP was measured from the upper nondominant arm at the brachial artery with an automated device (OMRON Standard BP Monitor HEM7203), via an appropriately sized cuff with the participant in a sitting position. Each measurement was taken twice with the average being used for analysis, and the same arm was used in the evening and morning.

\subsubsection{Blood Sampling}

A venous blood sample was taken from each participant near the time of the sleep study and again when admitted for delivery. Each sample was assayed for ET-1, sFlt-1, sEng and PIGF levels in maternal plasma were measured using Quantikine ELISA kits (R\&D Systems, Minneapolis, USA). Intraassay and interassay coefficients of variation were less than $10 \%$. In addition, the values of the quality controls provided in the kits read within the specified ranges.

\subsubsection{Medical Records for Maternal Outcomes}

Baseline information including maternal age, ethnicity, parity, BMI recorded at the first antenatal appointment and estimated due date of current pregnancy, as well as relevant comorbidities such as diagnosis of $\mathrm{GDM}^{297}$ were recorded at recruitment. After delivery, medical records were reviewed regarding diagnosis and severity of hypertensive disease, including gestation at diagnosis of GH or PE, days between diagnosis and delivery of the fetus, use of anti-hypertensive medication, and highest BPs recorded antenatally, postnatally and during the intrapartum period for those who laboured. BP recorded at each antenatal appointment was also recorded. Antenatal appointments were typically every four weeks during the second trimester increasing to every 2 weeks during the third trimester, however hypertensive participants were usually seen more frequently as clinically indicated. Participants with suspected or confirmed hypertension in pregnancy were routinely sent to the Pregnancy Day Assessment Centre for four-hour BP monitoring and blood sampling for indicators of PE severity - these pathology measures included quantification of proteinuria, serum liver transaminases, uric acid and creatinine, and platelet count. 
$\mathrm{GH}$ and PE was considered to be severe if $\mathrm{BP}$ was $>160 \mathrm{mmHg}$ systolic and/or $110 \mathrm{mmHg}$ diastolic on more than one occasion, and PE was considered as early-onset if diagnosed prior to 34 weeks gestation. ${ }^{298}$ Cases of GH or PE were reviewed by a senior obstetrician (Sue Walker) to confirm diagnosis.

\subsubsection{Procedures}

After written informed consent was obtained, participants with GH and PE underwent overnight PSG at their earliest convenience. Each control participant underwent PSG within \pm 4 weeks of gestational age of a matched case. A trained sleep technologist with over 10 years of experience (the candidate) was responsible for all sleep monitoring. At the time of the sleep study, height, weight and neck circumference were recorded, as well as BP.

Within \pm three weeks of the PSG (with $>80 \%$ of participants within one week), a venous blood sample was taken from each participant to coincide with routine antenatal blood sampling. Participants were also given a pack of blood tubes and pathology slips to take with them when admitted to have their baby. If admission for delivery was during business hours the research midwife collected another venous blood sample prior to the baby being delivered, otherwise routine hospital staff were relied upon for blood collection.

\subsubsection{Statistical Analysis}

All statistical analyses were performed with SPSS 21.0 (SPSS Inc., Chicago, Illinois). Data were assessed for linearity and normality by obtaining skewness and kurtosis values and by checking distributions on histograms and normal probability plots. Values are given in means with standard deviations $(M \pm S D)$ or median and interquartile range $(M d n(I Q R))$ for non-normally distributed variables. A two-sided $p$ value of less than 0.05 was considered to indicate statistical significance. SDB was defined as an RDI of $\geq 5$ events per hour, and the PE, GH and control participants were further split into the SDB group and the No SDB group. Supplementary analyses were performed with SDB defined as $R D I \geq 10$ and $R D I \geq 15$ to investigate the impact of more severe SDB on HDP.

Data analysis was done separately for the PE, GH and normotensive control participant groups in all analyses except the maternal blood markers where PE and GH were grouped together due to reduced sample size in consequence of missing data. Comparisons between the SDB and No SDB groups were done using Fisher's exact test of independence for categorical variables, independent-samples t-tests for normally-distributed continuous variables and Mann-Whitney $U$ tests for non-normally distributed continuous variables.

A sequential Cox regression survival analysis was performed to test the hypothesis that women with SDB will have a shorter gestation between diagnosis of a hypertensive disorder and delivery, than those 
without SDB, after adjusting for the effects of covariates with a p value of less than 0.10 on the log rank test. The assumption of proportional hazards was adequately met.

Mixed modelling was conducted to investigate change in systolic and diastolic BP across pregnancy for those with and without SDB, taking into account the risk factors and treatments for hypertension in pregnancy. BP measurements were noted from each antenatal appointment or Pregnancy Day Assessment Centre visit (first measurement from that visit) that was closest to the following gestations for the PE group = before 20 weeks, 24, 28, 30, 32, 34, and 35 weeks, for the $\mathrm{GH}$ group = before 20 weeks, 24, 28, 30, 32, 34, 35, 36, 37, and 38 weeks; and for the Control group = before 20 weeks, 24, $28,32,34,36,37,38$ and 39 weeks. The additional measurements of 30 and 35 weeks were available within the PE and GH group due to more frequent antenatal monitoring. Longitudinal BP data was not available for five control participants and one PE participant. Analysis was capped at the gestation after which more than half of the participants in that group had delivered. For the control group, data was missing for less than $15 \%$ of the participants between 20 - 34 weeks gestation and less than $25 \%$ for 36 - 38 weeks gestation. For the GH group, less than $25 \%$ of data was missing up until 37 weeks gestation. For the PE group, only $10 \%$ was missing from 20-28 weeks, however due to many premature deliveries around $40 \%$ of data was missing from $30-35$ weeks gestation (although the missing data was split evenly across SDB groups). Forward stepwise selection was used to construct a model using SDB and gestation as fixed factors and anti-hypertensive use at each gestation as a covariate. Other covariates tested in the model included BMI at first antenatal appointment, GDM, age, and parity; the explanatory variable with the smallest $p$ value (of less than 0.20 ) was added at each step.

BP measured at the first antenatal appointment was excluded from analysis if it was after 20 weeks gestation. Peak antenatal BP was calculated as the average of the highest three BP measurements during pregnancy. Biochemical measures of PE severity were not available on most of the control participants as the blood tests were only performed clinically for suspicion of hypertensive disease. Use of anti-hypertensive medication and gestation at which it was commenced was defined according to when the medication was first prescribed, or when first administered as an inpatient. Mixed betweenwithin subjects analysis of variance (ANOVA) was performed to compare change in BP from evening to morning on the sleep study night for the SDB and No SDB groups.

Maternal blood samples taken at the time of the sleep study ranged from $26+5$ to $37+5$ weeks; this sample was referred to as the 'third trimester' sample. The second sample taken at delivery was referred to as the 'delivery' sample. Blood samples that were unable to be collected were most commonly due to i) delivery overnight or on the weekend when the research study was dependent on the participant giving their blood pack to the hospital staff for blood sample collection, followed by timely collection and analysis of the sample, ii) precipitate labour (limited time to collect the sample) or iii) consent not given by the participant. As the maternal blood samples were taken at different gestations for each participant, a rate of change per week from the third trimester sample to the delivery sample was calculated to test 
the hypothesis that those with SDB as well as GH/PE would have increasingly worsening angiogenic markers of hypertensive disease.

After undergoing the PSG in the third trimester of pregnancy, three participants who were diagnosed with severe SDB commenced CPAP - one participant with PE (RDI of 150.6) and two control participants (RDI of 108.8 and 83.6). The PE participant was left in analyses regarding diagnosis of hypertensive disease as CPAP was commenced after PE diagnosis. This participant was also left in the analyses for severity of hypertension as she had severe hypertension and was administered medication despite CPAP use. However, she was removed from the analysis regarding time from diagnosis of PE to delivery. The three participants on CPAP were included in the longitudinal analysis of $\mathrm{BP}$ however the $\mathrm{BP}$ values after commencing CPAP were censored, and they were excluded from the analysis of the maternal blood samples.

\subsection{Results}

\subsubsection{Demographics}

A total of 87 pregnant women participated, with one study failure in the control group due to substantial signal loss during the sleep study, and one control participant excluded as she changed care providers five weeks prior to delivery.

As per Table $4.1,41 \%$ of the PE group, $63 \%$ of the GH group and $39 \%$ of the control group had SDB. Within each group, there were no differences in age, parity, GDM, and gestation at sleep study for the SDB versus No SDB groups. The BMI value measured at the first antenatal appointment was taken at a mean of $15.1 \pm 2.6$ weeks gestation; neither this nor BMI at the sleep study appointment were different across groups. By design, those with SDB had a significantly higher RDI, ODI3\% and ODI4\% than those without SDB (Table 4.1).

An in-laboratory sleep study was chosen by 25 participants and a home sleep study chosen by 41 participants, the remaining 19 participants were inpatients (with no difference in RDI, in-lab $M d n(I Q R)$ $=4.6(3.7-18.2)$ vs. home $=4.0(2.3-9.2)$ vs. inpatient $=4.5(1.8-12.3), p=0.48)$. At the time of the sleep study, 23 of the hypertensive participants were taking anti-hypertensive medication; 16 were taking Labetalol, five were taking Aldomet, one was taking both Labetalol and Aldomet, and one was taking both Labetalol and Nifedipine. 
Table 4.1 Demographics for Preeclampsia and Gestational Hypertension Cases and Normotensive Control Groups Stratified by SDB Status

\begin{tabular}{|c|c|c|c|c|c|c|c|c|c|}
\hline & \multicolumn{2}{|c|}{$\begin{array}{c}\text { PE } \\
(n=17)\end{array}$} & \multicolumn{3}{|c|}{$\begin{array}{c}\mathrm{GH} \\
(\mathrm{n}=24)\end{array}$} & \multicolumn{4}{|c|}{$\begin{array}{l}\text { Controls } \\
(n=44)\end{array}$} \\
\hline & $\begin{array}{c}\text { SDB } \\
(\mathrm{n}=7)\end{array}$ & $\begin{array}{l}\text { No SDB } \\
(n=10)\end{array}$ & $\mathrm{p}$ & $\begin{array}{c}\text { SDB } \\
(n=15)\end{array}$ & $\begin{array}{c}\text { No SDB } \\
(n=9)\end{array}$ & $\mathrm{p}$ & $\begin{array}{c}\text { SDB } \\
(n=17)\end{array}$ & $\begin{array}{l}\text { No SDB } \\
(n=27)\end{array}$ & $p$ \\
\hline Age (years) & $30.6 \pm 4.7$ & $32.0 \pm 4.9$ & .56 & $33.9 \pm 5.1$ & $32.0 \pm 4.7$ & .35 & $33.4 \pm 3.8$ & $33.1 \pm 4.7$ & .85 \\
\hline Nulliparous & $6(85.7 \%)$ & $7(70.0 \%)$ & .60 & $8(53.3 \%)$ & $5(55.6 \%)$ & 1.0 & $10(58.8 \%)$ & $13(48.1 \%)$ & .55 \\
\hline GDM & $3(41.2 \%)$ & $3(30.0 \%)$ & .64 & $2(13.3 \%)$ & $0(0.0 \%)$ & .51 & $4(23.5 \%)$ & $4(14.8 \%)$ & .69 \\
\hline BMI first appt & $33.1 \pm 9.6$ & $29.7 \pm 8.3$ & .45 & $34.4 \pm 5.5$ & $31.8 \pm 6.5$ & .31 & $33.9 \pm 7.6$ & $32.2 \pm 6.7$ & .44 \\
\hline Weight gain $(\mathrm{kg})$ & $9.9 \pm 5.8$ & $7.3 \pm 5.8$ & .38 & $12.1 \pm 4.5$ & $10.1 \pm 7.0$ & .40 & $9.9 \pm 5.8$ & $9.0 \pm 5.4$ & .58 \\
\hline BMI PSG & $37.3 \pm 9.1$ & $32.3 \pm 7.8$ & .24 & $38.7 \pm 4.7$ & $35.4 \pm 6.1$ & .15 & $37.6 \pm 6.7$ & $35.4 \pm 5.7$ & .24 \\
\hline Gest PSG (weeks) & $30.6 \pm 3.5$ & $31.8 \pm 3.0$ & .45 & $35.5 \pm 1.6$ & $34.5 \pm 3.8$ & .48 & $33.1 \pm 2.2$ & $32.5 \pm 3.2$ & .48 \\
\hline $\mathrm{RDI} / \mathrm{hr}$ & $\begin{array}{c}16.8 \\
(12.3,108.6)\end{array}$ & $1.9(1.5,3.0)$ & $<.001$ & $\begin{array}{c}12.5 \\
(7.2,26.1)\end{array}$ & $3.0(1.1,3.2)$ & $<.001$ & $8.8(7.2,27.3)$ & $2.9(1.5,4.0)$ & $<.001$ \\
\hline ODI $\geq 3 \%$ overall & $\begin{array}{c}6.0 \\
(0.4,109.7)\end{array}$ & $0.7(0.2,1.4)$ & .055 & $5.9(3.2,35.9)$ & $1.3(0.0,2.9)$ & .002 & $5.4(0.7,27.1)$ & $1.0(0.2,2.4)$ & .007 \\
\hline ODI $\geq 4 \%$ overall & $\begin{array}{c}1.9 \\
(0.0,101.0)\end{array}$ & $0.1(0.0,0.7)$ & .055 & $3.3(0.5,23.3)$ & $0.3(0.0,1.3)$ & .01 & $1.9(0.3,12.7)$ & $0.3(0.0,0.5)$ & .005 \\
\hline
\end{tabular}

Note. Values given as $\mathrm{M} \pm \mathrm{SD}$, Mdn (IQR), or $\mathrm{n}(\%) . \mathrm{PE}=$ preeclampsia, GH = gestational hypertension, SDB = sleep-disordered breathing, GDM = gestational diabetes mellitus, $\mathrm{BMI}=$ body mass index $, \mathrm{kg} / \mathrm{m}^{2}, \mathrm{PSG}=$ polysomnography, $\mathrm{RDI}=$ respiratory disturbance index, $\mathrm{ODI}=$ oxygen desaturation index. Weight gain measured from first appointment to PSG. 


\subsubsection{Hypertensive Disease and Blood Pressure Control}

\subsubsection{Preeclampsia Participants}

\subsection{Gestational Age at Diagnosis of Preeclampsia}

Of the 17 women who had PE at the time of the sleep study, there was no significant difference in the average gestation at diagnosis of PE for those with and without SDB (SDB $=29.1$ weeks \pm 3.7 vs No $\mathrm{SDB}=31.0 \pm 3.6, \mathrm{p}=.32$ ). Fourteen of the PE group were diagnosed as early-onset ( $<34$ weeks $)$, again there was no difference between those with SDB and those in the No SDB group $(85.7 \%$ vs. $80.0 \%, p=1.0$ ). Ten of the 17 women had FGR as part of their PE diagnosis (four in the SDB group $(57.1 \%)$ versus six in the No SDB group $(60.0 \%), p=1.0)$.

\subsection{Severity of Preeclampsia}

There was no significant difference in gestational age at delivery between the SDB and no SDB group $(\mathrm{SDB}=32.1$ weeks \pm 4.8 vs No SDB $=33.0 \pm 3.5, p=.68)$. Given there was no difference in gestational age at diagnosis between the groups, we used another index of disease severity: the number of days between diagnosis of PE and delivery comparing those with and without SDB. The survival curve (Figure 4.1) demonstrates that the SDB group had $21.0 \pm 17.5$ days from diagnosis to delivery compared to $13.3 \pm 8.1$ days for the No SDB group $(p=.25)$. With consideration of potential covariates relating to preterm birth, SDB had no statistically significant effect on the number of days between PE diagnosis and delivery after taking into account known FGR at the sleep study, $X^{2}(1)=1.17, \mathrm{OR} 0.52,95 \% \mathrm{Cl}$ $[0.16,1.71], p=.28$, Figure 4.1. There was a trend for those with FGR to have a higher risk of earlier delivery following PE diagnosis, $X^{2}(1)=3.08$, OR 3.25, 95\% Cl $[0.87,12.12], p=.08$.

Of the 17 participants with PE, 12 had hypertension classified as severe (systolic BP $\geq 160 \mathrm{mmHg}$ and/or diastolic BP $\geq 110 \mathrm{mmHg}$ ) by the end of their pregnancy - four of those had SDB (4/7 $=57.1 \%)$ and 8 of those did not $(8 / 10=80.0 \% ; p=.59)$. Fourteen of the 17 participants with $P E$ required anti-hypertensive medication during their pregnancy - $5(71.4 \%)$ were in the SDB group and $9(90.0 \%)$ were in the No SDB group $(p=.54)$. There was no difference in the average gestation that anti-hypertensives were commenced $(\mathrm{SDB}=28.3$ weeks \pm 5.3 vs No SDB $=31.6 \pm 3.5, p=.23$ ). Multiple anti-hypertensives tended to be administered more often in the No SDB group (80.0\%) compared to the SDB group (28.6\%, $\mathrm{p}=.06)$. 


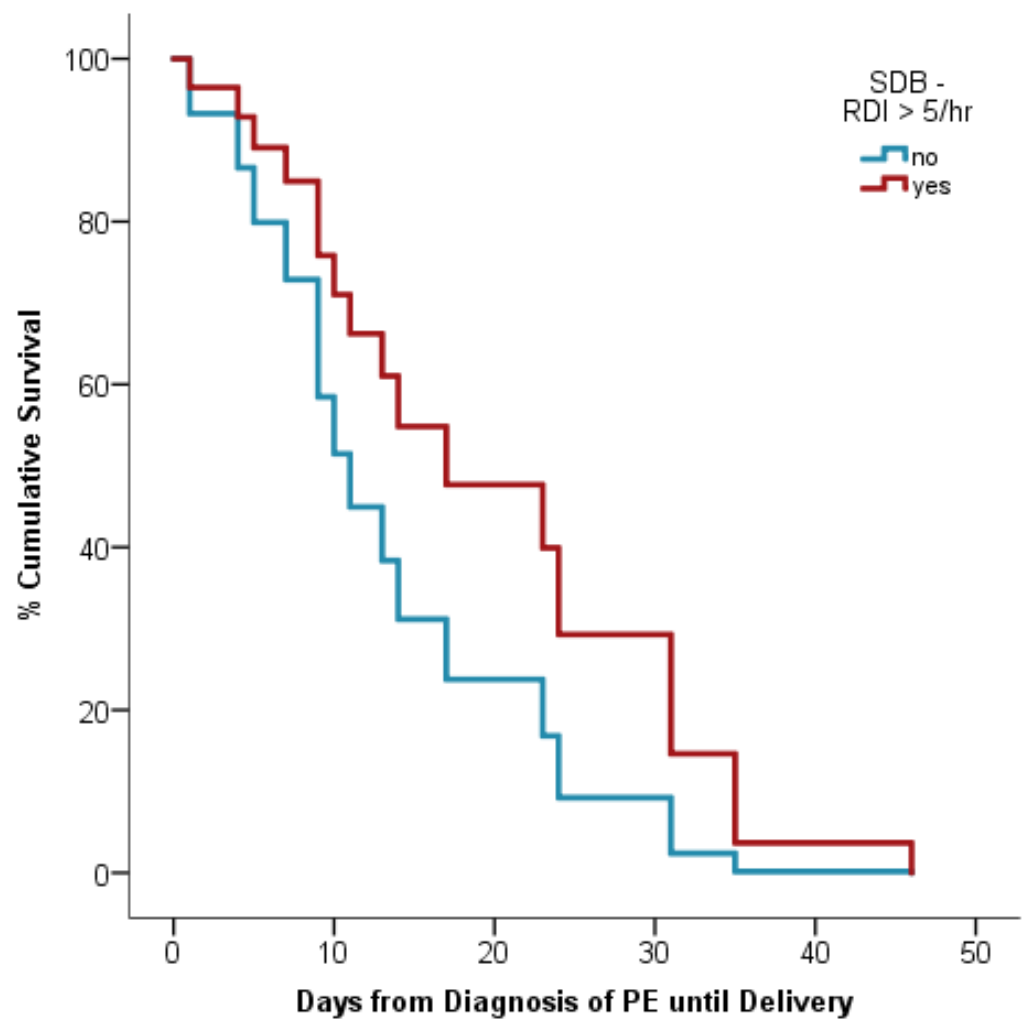

Figure 4.1 Survival curve for the number of days from diagnosis of preeclampsia (PE) until delivery, with the covariate of fetal growth restriction (FGR), for the $S D B(n=6)$ and No SDB $(n=10)$ groups. $S D B=$ sleep-disordered breathing, $R D I=$ respiratory disturbance index .

\subsection{Blood Pressure across Pregnancy - Preeclampsia}

Mixed modelling was conducted to investigate change in BP across pregnancy for those with and without SDB, taking into account risk factors and treatment for hypertension in pregnancy.

Within the PE group (Figure 4.2), systolic BP rose by $14.8 \pm 7.6 \mathrm{mmHg}$ on average across pregnancy, however this was not statistically significant $(p=.11)$, and there was no effect of SDB on systolic BP ( $p$ $=.63$ ) or interaction between SDB group and gestation $(p=.15)$. Anti-hypertensive use was a significant factor; when medication was administered systolic BP was estimated as $12.9 \mathrm{mmHg}(95 \% \mathrm{Cl}$ [2.72, 23.0] higher than when not required $(p=.01)$.

The increase in diastolic BP across pregnancy was not significant $(15.2 \pm 5.5 \mathrm{mmHg}, p=0.10)$ and there was no effect of SDB on diastolic BP $(p=.40)$ and no interaction $(p=.42)$. Anti-hypertensive use was not a significant covariate in this model $(p=.12)$. No other covariates met criteria to be entered into the model for systolic or diastolic BP. 


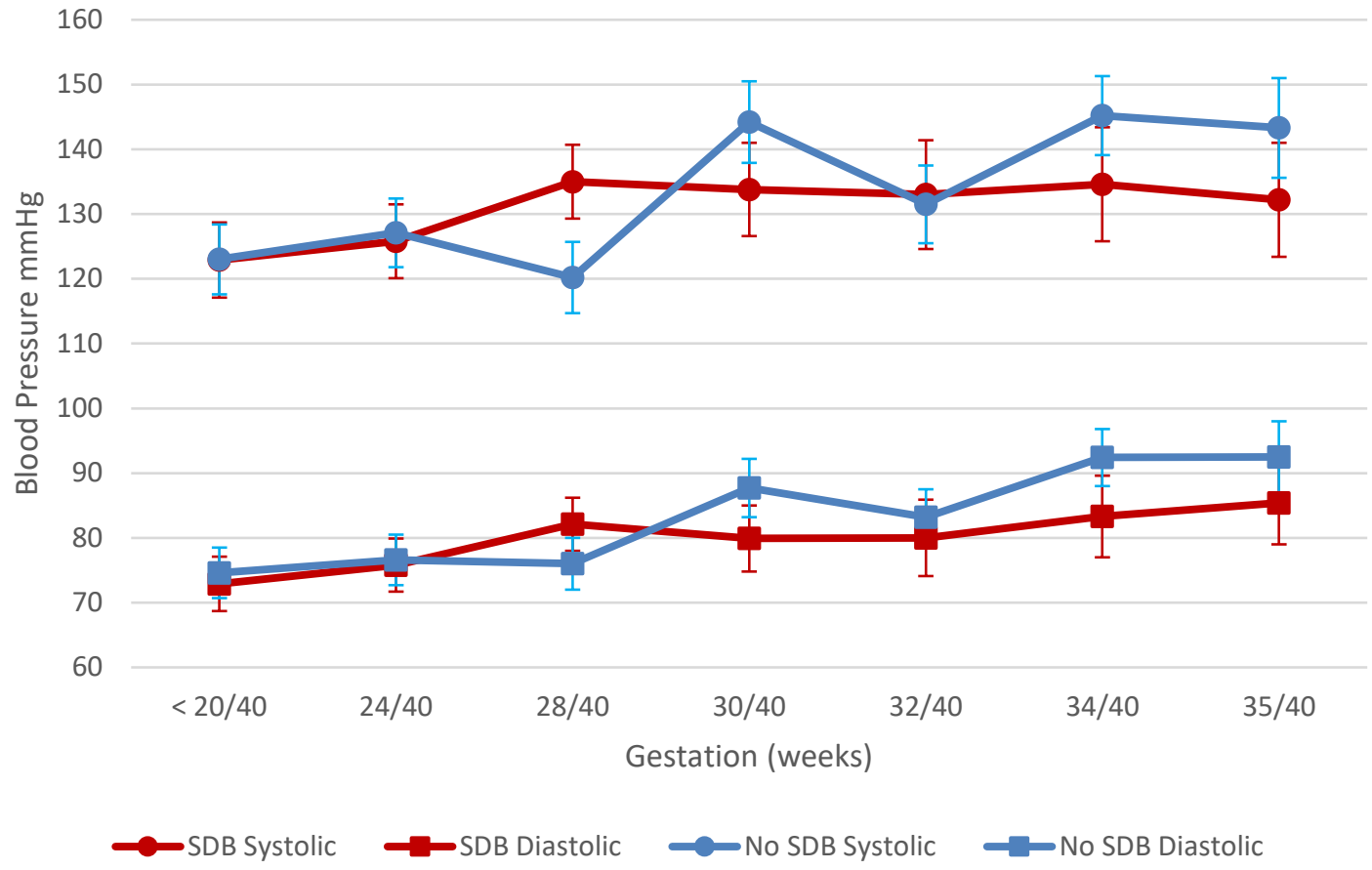

Figure 4.2 Mean \pm SEM systolic and diastolic blood pressure across pregnancy for the SDB group $(n=$ 7) and the No SDB group $(n=9)$ within the preeclampsia group. Values based on parameter estimates for the autoregressive mixed model with covariate of antihypertensive use. SDB = sleep-disordered breathing.

Peak measures of BP during pregnancy and postpartum for the PE group are presented in Table 4.2. There was no difference in systolic or diastolic BP at first antenatal visit between those with or without SDB. Women with PE and SDB did not have a higher peak BP antenatally or postnatally compared to women without SDB. 
Table 4.2 Booking and Peak Blood Pressure for Preeclampsia and Gestational Hypertension Cases and Normotensive Control Groups Stratified by SDB Status

\begin{tabular}{|c|c|c|c|c|c|c|c|c|c|}
\hline & \multicolumn{2}{|c|}{$\begin{array}{c}\text { PE } \\
(n=17)\end{array}$} & \multirow[b]{2}{*}{$\mathrm{p}$} & \multicolumn{2}{|c|}{$\begin{array}{c}\text { GH } \\
(n=24)\end{array}$} & \multicolumn{4}{|c|}{$\begin{array}{l}\text { Controls } \\
(\mathrm{n}=39)\end{array}$} \\
\hline & $\begin{array}{l}\text { SDB } \\
(n=7)\end{array}$ & $\begin{array}{l}\text { No SDB } \\
(n=10)\end{array}$ & & $\begin{array}{c}\text { SDB } \\
(n=15)\end{array}$ & $\begin{array}{c}\text { No SDB } \\
(n=9)\end{array}$ & $p$ & $\begin{array}{c}\text { SDB } \\
(n=15)\end{array}$ & $\begin{array}{l}\text { No SDB } \\
(n=24)\end{array}$ & $p$ \\
\hline \multicolumn{10}{|l|}{ First visit $\mathrm{BP}^{\mathrm{a}}$} \\
\hline Systolic mmHg & $119.0 \pm 10.6$ & $117.3 \pm 14.2$ & .79 & $119.3 \pm 10.3$ & $120.8 \pm 12.4$ & .76 & $110.8 \pm 11.7$ & $113.3 \pm 11.5$ & .53 \\
\hline Diastolic $\mathrm{mmHg}$ & $72.9 \pm 11.1$ & $71.4 \pm 10.0$ & .79 & $74.0 \pm 8.3$ & $75.3 \pm 9.0$ & .72 & $67.5 \pm 8.8$ & $66.8 \pm 6.8$ & .79 \\
\hline \multicolumn{10}{|l|}{ Peak BP } \\
\hline Systolic $\mathrm{mmHg}$ & $163.1 \pm 11.7$ & $166.9 \pm 11.2$ & .51 & $155.2 \pm 5.2$ & $161.7 \pm 14.8$ & .13 & $127.0 \pm 10.7$ & $128.7 \pm 9.5$ & .60 \\
\hline Diastolic $\mathrm{mmHg}$ & $103.1 \pm 5.2$ & $102.4 \pm 5.4$ & .78 & $100.3 \pm 6.1$ & $102.4 \pm 7.2$ & .45 & $79.4 \pm 7.4$ & $80.2 \pm 7.5$ & .76 \\
\hline \multicolumn{10}{|l|}{ Increase in $\mathrm{BPa}$} \\
\hline Systolic mmHg & $44.1 \pm 12.8$ & $48.9 \pm 14.4$ & .52 & $35.9 \pm 9.0$ & $40.9 \pm 19.5$ & .40 & $16.9 \pm 10.0$ & $15.4 \pm 13.2$ & .72 \\
\hline Diastolic $\mathrm{mmHg}$ & $30.3 \pm 12.2$ & $30.4 \pm 12.9$ & .99 & $26.3 \pm 11.5$ & $27.1 \pm 13.2$ & .88 & $12.5 \pm 7.0$ & $13.4 \pm 7.7$ & .71 \\
\hline \multicolumn{10}{|l|}{ Peak IP BPb } \\
\hline Systolic mmHg & * & * & * & $137.9 \pm 18.0$ & $158.2 \pm 2.5$ & .02 & $133.3 \pm 17.5$ & $130.8 \pm 12.8$ & .68 \\
\hline Diastolic $\mathrm{mmHg}$ & * & * & * & $87.1 \pm 12.5$ & $96.8 \pm 4.1$ & .10 & $81.7 \pm 7.5$ & $79.0 \pm 8.9$ & .46 \\
\hline \multicolumn{10}{|l|}{ Peak PN BP } \\
\hline Systolic mmHg & $158.7 \pm 14.1$ & $161.0 \pm 12.6$ & .74 & $154.1 \pm 19.0$ & $162.9 \pm 16.7$ & .26 & $133.1 \pm 15.5$ & $131.5 \pm 13.2$ & .73 \\
\hline Diastolic $\mathrm{mmHg}$ & $98.3 \pm 8.6$ & $97.3 \pm 7.8$ & .81 & $95.8 \pm 8.8$ & $98.8 \pm 9.0$ & .43 & $88.1 \pm 13.1$ & $80.8 \pm 9.5$ & .051 \\
\hline
\end{tabular}

Note. Values given as $\mathrm{M} \pm \mathrm{SD} . \mathrm{PE}=$ preeclampsia, $\mathrm{GH}=$ gestational hypertension, $\mathrm{SDB}=$ sleep-disordered breathing, $\mathrm{BP}=$ blood pressure, IP $=$ intrapartum, $\mathrm{PN}=$ postnatal.

aFour participants (two in PE no SDB group; two in Control SDB group) excluded as first visit was after 20 weeks gestation.

bIntrapartum BP recorded only for those who laboured $-\mathrm{GH} \operatorname{SDB} n=7, \mathrm{GH}$ no $\operatorname{SDB} n=5$, Controls $\operatorname{SDB} n=9$, Controls no SDB $n=16$. Insufficient numbers for PE group - 2 vs 3. 


\subsection{Biochemical and Haematological Indicators of Disease Severity}

There were no differences in the worst biochemical and haematological markers of disease for the pregnancy (proteinuria, ALT, urate, creatinine and platelets) when comparing those who had SDB and those who did not (Table 4.3).

Table 4.3 Biochemical and Haematological Measures of Preeclampsia Severity for the Preeclampsia and Gestational Hypertension Group Stratified by SDB Status

\begin{tabular}{lcccccc}
\hline & \multicolumn{3}{c}{ PE } & \multicolumn{3}{c}{$\mathrm{GH}$} \\
\cline { 2 - 7 } & $\begin{array}{c}\text { SDB } \\
(\mathrm{n}=7)\end{array}$ & $\begin{array}{c}\text { No SDB } \\
(\mathrm{n}=10)\end{array}$ & $\mathrm{p}$ & $\begin{array}{c}\text { SDB } \\
(\mathrm{n}=15)\end{array}$ & $\begin{array}{c}\text { No SDB } \\
(\mathrm{n}=9)\end{array}$ & $\mathrm{P}$ \\
\hline Peak Pr:cr ratio & $.10(.05, .31)$ & $.15(.05, .38)$ & .81 & $.03(.02, .08)$ & $.02(.02, .03)$ & .12 \\
Peak ALT & 36.0 & 23.0 & .36 & 24.0 & 20.0 & .91 \\
& $(15.0,63.0)$ & $(13.0,29.8)$ & & $(15.0,32.0)$ & $(16.0,42.5)$ & \\
Peak Urate & $0.44 \pm 0.12$ & $0.46 \pm 0.12$ & .75 & $0.34 \pm 0.06$ & $0.34 \pm 0.04$ & .95 \\
Peak Creatinine & $69.7 \pm 19.0$ & $71.6 \pm 15.1$ & .82 & $60.0 \pm 13.8$ & $61.8 \pm 6.0$ & .72 \\
Nadir Platelets & $202.1 \pm 48.1$ & $188.5 \pm 53.1$ & .60 & $213.7 \pm 48.3$ & $191.7 \pm 61.9$ & .34
\end{tabular}

Note. Values given as $\mathrm{M} \pm \mathrm{SD}$ or Mdn (IQR). $\mathrm{PE}=$ preeclampsia, $\mathrm{GH}=$ gestational hypertension, SDB = sleep-disordered breathing, $\operatorname{Pr}: \mathrm{cr}=$ protein:creatinine, $\mathrm{ALT}=$ alanine transaminase .

\subsection{Overnight Blood Pressure on Sleep Study - Preeclampsia}

For the PE group (Figure 4.3), there was no change in systolic BP from evening to morning overall $[F(1,14)=0.58, p=.46]$, and the rate of BP change overnight did not differ for each SDB group $[F(1,14)$ $=0.00, p=1.0]$. Overall, those with SDB did not have a higher overall systolic BP than those without $\operatorname{SDB}[F(1,14)=0.01, p=.93]$. There were no differences in diastolic BP overnight (main effects; time $=$ $[F(1,14)=0.26, p=.62], S D B=[F(1,14)=0.21, p=.66]$, interaction $=[F(1,14)=0.40, p=.54])$. 


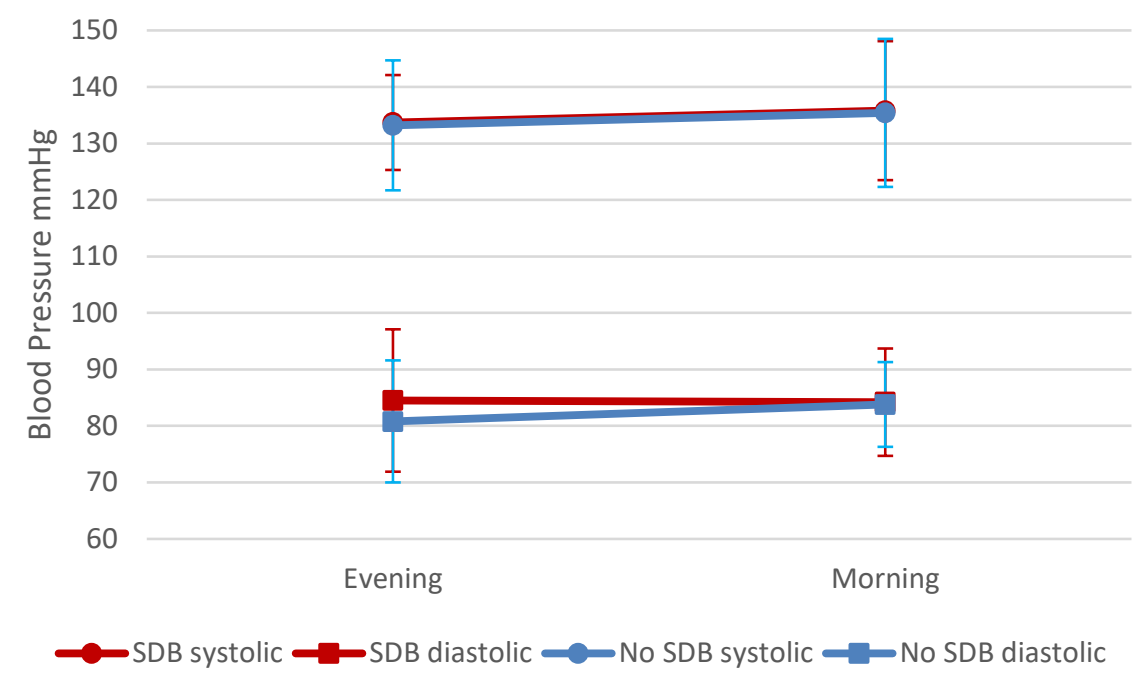

Figure 4.3 Mean \pm SEM overnight systolic and diastolic blood pressure on the night of the sleep study for the PE group, for those with and without $S D B . S D B=$ sleep-disordered breathing.

\subsubsection{Gestational Hypertension Participants}

\subsection{Gestation at Diagnosis of Gestational Hypertension}

There was no difference in the average gestation at diagnosis of $\mathrm{GH}$ for those with and without SDB $(\mathrm{SDB}=33.2$ weeks \pm 2.2 vs No SDB $=32.5 \pm 3.9, \mathrm{p}=.59$ ).

\subsection{Severity of Gestational Hypertension}

There was no significant difference in gestational age at delivery between the SDB and no SDB group $(\mathrm{SDB}=37.8$ weeks \pm 2.0 vs No $\mathrm{SDB}=37.0 \pm 2.9, \mathrm{p}=.46)$. Figure 4.4 shows the survival curve for the number of days between diagnosis of $\mathrm{GH}$ and delivery, comparing those with and without SDB. On average, the SDB group had $32.1 \pm 21.3$ days from diagnosis to delivery compared to $31.8 \pm 12.9$ days for the No SDB group $(p=.97)$.

Gestational age at diagnosis was a significant predictor of the number of days between diagnosis of GH and delivery, $X^{2}(1)=8.28, p=.004$; for each week of advancing gestational age the risk of delivery increased by $36 \%$ (OR 1.36, 95\% $\mathrm{Cl}[1.10,1.68])$. This is unsurprising since the threshold for delivery falls as gestation increases. To account for this, we controlled for the effect of gestational age at diagnosis on the number of days until delivery. SDB had no statistically significant effect on the number of days between $\mathrm{GH}$ diagnosis and delivery after taking into account gestational age at GH diagnosis, $\mathrm{X}^{2}(1)=0.93$, OR $0.64,95 \% \mathrm{Cl}[0.26,1.58], \mathrm{p}=.34$, Figure 4.4 . 


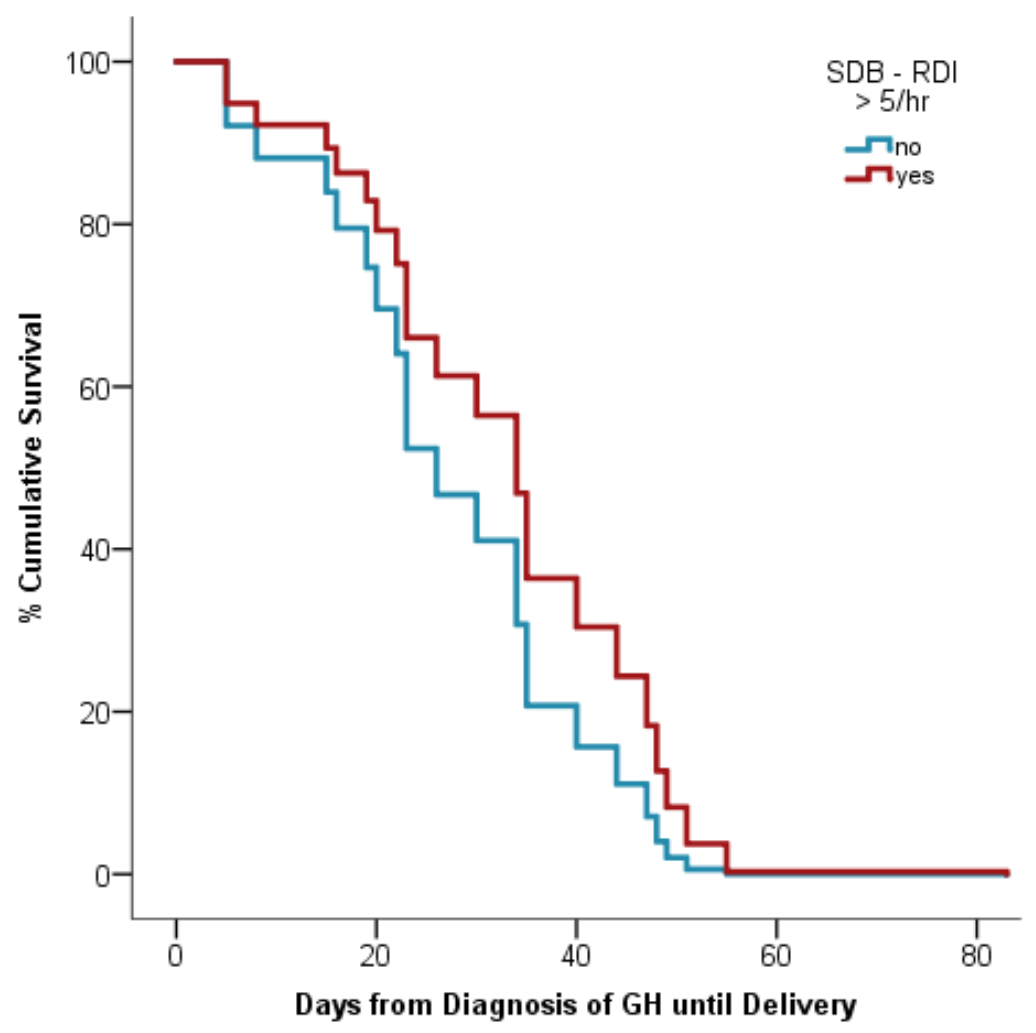

Figure 4.4 Survival curve for the number of days between diagnosis of gestational hypertension (GH) and delivery with gestation of diagnosis of $\mathrm{GH}$ as a covariate, for the $S D B(n=15)$ and No $S D B(n=9)$ groups. $S D B=$ sleep-disordered breathing, $R D I=$ respiratory disturbance index .

Of the $24 \mathrm{GH}$ participants at the time of the sleep study, 5/15 with SDB (33.3\%) went on to develop PE compared with $3 / 9$ without $\operatorname{SDB}(33.3 \%, p=1.0)$. Of these 8 women with $P E$, the 2 who were early onset were both within the No SDB group $(66.7 \%$ vs. $0 \%, p=.11)$.

Four of the 15 with SDB (26.7\%) and 4 of the 9 without SDB (44.4\%) had hypertension classified as severe (systolic BP $\geq 160 \mathrm{mmHg}$ and/or diastolic BP $\geq 110 \mathrm{mmHg}$ ) by the end of their pregnancy $(p=$ .41). Twenty of the $24 \mathrm{GH}$ women required anti-hypertensive medication during their pregnancy -12 $(80.0 \%)$ were in the SDB group and $8(88.9 \%)$ were in the No SDB group $(p=1.0)$. There was no difference in the average gestation that anti-hypertensives were commenced (SDB $=35.1$ weeks \pm 3.6 vs No SDB $=33.1 \pm 4.6, p=.30$ ). There was no difference in the frequency that multiple antihypertensives were needed between the SDB group (13.3\%) and No SDB group either $(11.1 \%, p=$ 1.0). 


\subsection{Blood Pressure across Pregnancy - Gestational Hypertension}

Within the mixed model for the GH group (Figure 4.5), systolic BP increased significantly across pregnancy (increase of $20.6 \pm 4.5 \mathrm{mmHg}, \mathrm{p}<.001$ ). There was also a trend toward the SDB group having a lower average systolic BP than the no SDB group $(130.0 \pm 1.5 \mathrm{mmHg}$ vs $134.2 \pm 1.98 \mathrm{mmHg}$, $p=.10)$, but there was no interaction between SDB group and gestation $(p=.48)$.

Diastolic BP also increased significantly across pregnancy (increase of $14.4 \pm 3.7 \mathrm{mmHg}, \mathrm{p}<.001$ ) and was significantly higher in the No SDB group compared to the SDB group $(85.1 \pm 1.6 \mathrm{mmHg}$ vs $80.5 \pm$ $1.2 \mathrm{mmHg}, p=.045)$. There was no interaction between SDB group and gestation for diastolic BP $(p=$ .64). Parity was a significant predictor in the model - nulliparous women had an estimated increase of $4.8 \mathrm{mmHg}(95 \% \mathrm{Cl}[0.14,9.39])$ in diastolic BP compared to multiparous women $(p=.04)$. BMI was included in the model for diastolic BP but was not significant $(p=.27)$. Medication was not a significant covariate in the model for systolic or diastolic BP $(p=0.79$ and 0.14$)$.

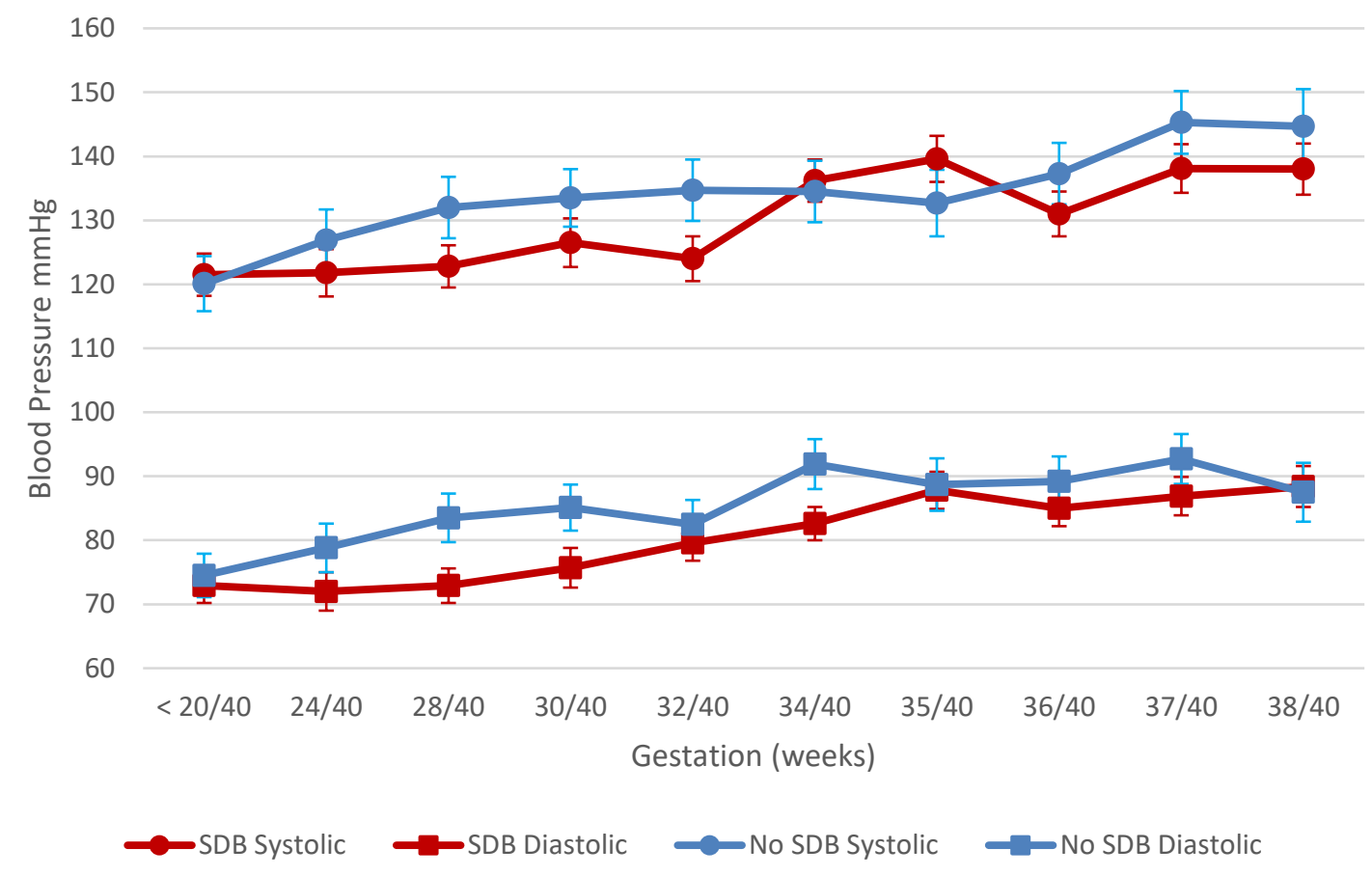

Figure 4.5 Mean \pm SEM systolic and diastolic blood pressure (BP) across pregnancy for the SDB group $(n=15)$ and the No SDB group ( $n=9)$ within the gestational hypertension (GH) group. Values based on parameter estimates for the mixed model with covariates of antihypertensive use for systolic BP, and antihypertensive use, parity and BMI for diastolic $B P . S D B=$ sleep-disordered breathing. 
Peak measures of BP throughout pregnancy for the GH group are presented in Table 4.2. There was no difference in systolic or diastolic BP at first antenatal visit between those with or without SDB. Women with GH and SDB did not have a higher peak BP antenatally or postnatally compared to women without SDB. Instead, women in the No SDB group had a significantly higher systolic BP in the intrapartum period compared to those with SDB $(p=.02)$.

\subsection{Biochemical and Haematological Indicators of Disease Severity}

As shown in Table 4.3, there were no differences in the markers of hypertensive disease severity (peak pr:cr ratio, ALT, urate, creatinine and nadir platelets) for the GH group, between those who had SDB and those who did not.

\subsection{Overnight Blood Pressure on Sleep Study - Gestational Hypertension}

In terms of systolic BP (Figure 4.6), there was no change from evening to morning overall $[F(1,19)=$ $1.95, p=.18]$, and no difference between those with and without $\operatorname{SDB}[F(1,19)=0.03, p=.87]$. The rate of $B P$ change overnight tended to be different for each SDB group $[F(1,19)=3.50, p=.077]$, with systolic BP increasing by $1.1 \mathrm{mmHg}$ for the SDB group and decreasing by $7.4 \mathrm{mmHg}$ for the No SDB group. There were no differences in diastolic BP overnight (main effects; time $=[F(1,19)=0.55, p=$ $.47], \mathrm{SDB}=[F(1,19)=0.02, p=.91]$, interaction $=[F(1,19)=0.14, p=.71])$.

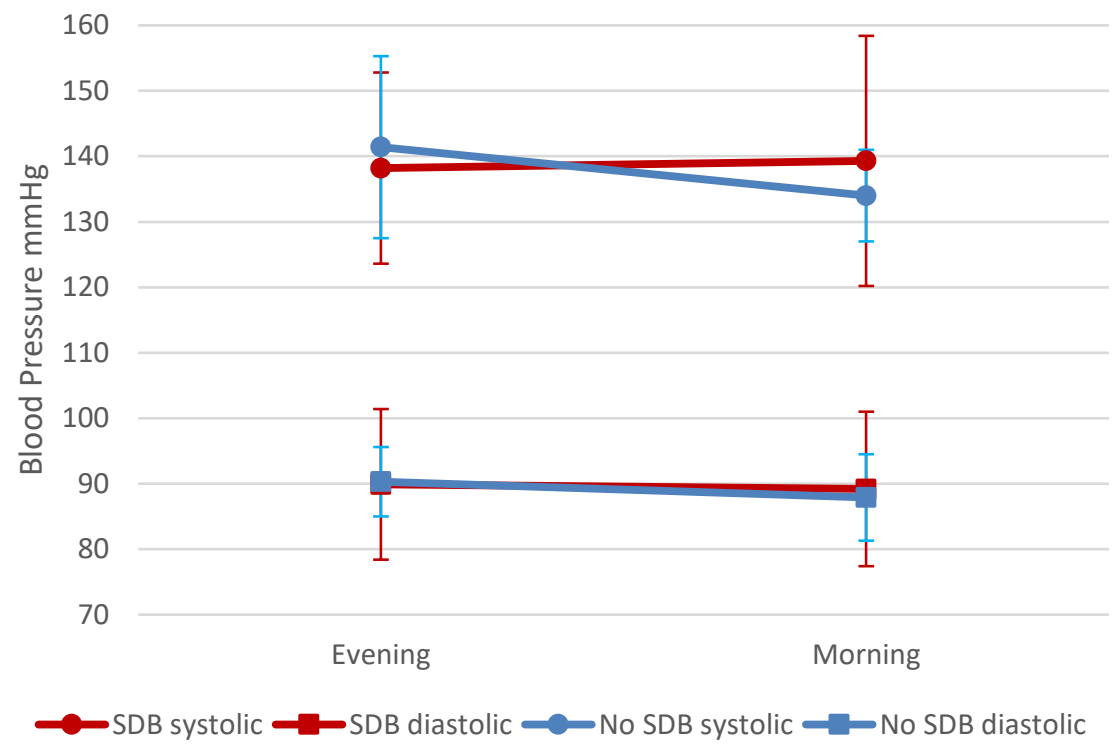

Figure 4.6 Mean \pm SEM overnight blood pressure on the night of the sleep study for the gestational hypertension $(G H)$ group, for those with and without $S D B$. SDB = sleep-disordered breathing. 


\subsubsection{Control Participants}

\subsection{Diagnosis of Hypertensive Disorders of Pregnancy}

Of the 44 participants who were normotensive at the time of the sleep study, 1 with SDB (5.9\%) was later diagnosed with $\mathrm{GH}$ versus 2 without SDB $(7.4 \%, \mathrm{p}=.85)$. Neither of the two control participants using CPAP developed $\mathrm{GH}$. None of the normotensive patients later developed PE.

\subsection{Severity of Hypertensive Disease}

There was no difference in gestational age at delivery between the SDB and no SDB group (SDB = 39.1 weeks \pm 1.8 vs No SDB $=38.7 \pm 3.3, p=.68$ ). None of the three control participants later diagnosed with $\mathrm{GH}$ had severe hypertension. Only one participant (in the No SDB group) used antihypertensive medication, administered during labour.

\subsection{Blood Pressure across Pregnancy - Controls}

Change in BP across pregnancy for those with and without SDB in the control group, taking into account risk factors for hypertension in pregnancy, is shown in Figure 4.7.

Systolic BP increased significantly across pregnancy (increase of $12.5 \pm 2.6 \mathrm{mmHg}, p<.001$ ). There was no effect of SDB on systolic BP $(p=.15)$ however the interaction between SDB group and gestation was significant $(p=.03)$. Specifically, between 38 and 39 weeks gestation there was a sharp increase in systolic BP of $17.7 \mathrm{mmHg}$ in the SDB group compared to an increase of only $1.4 \mathrm{mmHg}$ in the No SDB group. BMI at the first antenatal visit was a significant covariate, with an average increase in systolic $\mathrm{BP}$ of $0.42 \mathrm{mmHg}(95 \% \mathrm{Cl}[0.18,0.66])$ per unit of $\mathrm{BMI}(p=.001)$.

Diastolic BP also increased significantly across pregnancy (increase of $9.9 \pm 2.1 \mathrm{mmHg}, \mathrm{p}<.001$ ), but there was no effect of SDB group on diastolic BP $(p=.58)$ and there was no interaction between SDB group and gestation $(\mathrm{p}=.25)$. BMI at the first antenatal appointment was again a significant covariate, with an estimated increase in diastolic BP of $0.49 \mathrm{mmHg}(95 \% \mathrm{Cl}[0.29,0.68])$ per unit of BMI $(p<.001)$. Age was included in the model for diastolic BP but was not significant $(p=.10)$. No other covariates for systolic and diastolic BP were included in the models. 


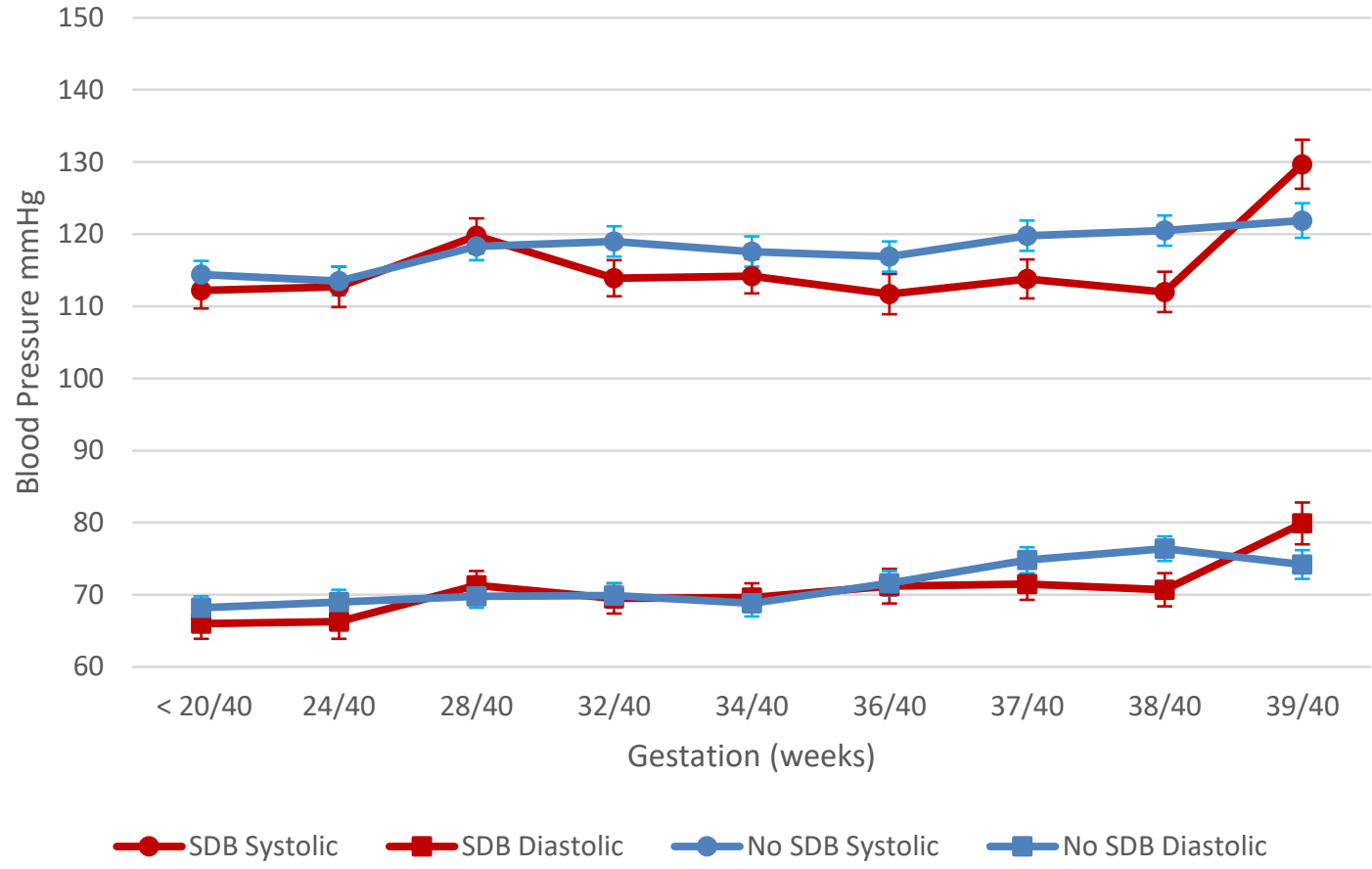

Figure 4.7 Mean \pm SEM systolic and diastolic blood pressure $(B P)$ across pregnancy for the SDB group $(n=15)$ and the No SDB group $(n=24)$ within the control group. Values based on parameter estimates for the mixed model with covariate of BMI for systolic BP, and BMI and age for diastolic BP. SDB = sleep-disordered breathing.

Peak measures of BP throughout pregnancy for the control group are presented in Table 4.2. There was no difference in systolic or diastolic BP at first antenatal visit between those with or without SDB. Women with SDB did not have a higher peak BP antenatally or during the intrapartum period, compared to women without SDB. Peak systolic BP postnatally did not differ across the SDB groups, however, there was a trend for those with SDB to have a higher peak diastolic BP during the postnatal period compared to the No SDB group $(p=.051)$.

\subsection{Overnight Blood Pressure on Sleep Study - Controls}

On the night of the sleep study, there was no change in systolic BP from evening to morning (Figure 4.8, $[F(1,30)=0.88, p=.36])$, or in rate of change overnight for each $\operatorname{SDB}$ group $[F(1,30)=0.81, p=$ .38]. Those with SDB did not have a higher overall BP than those without $\operatorname{SDB}[F(1,30)=0.49, p=.49]$. In terms of diastolic $B P$, there were also no differences (main effects; time $=[F(1,30)=0.002, p=.97]$, $\operatorname{SDB}=[F(1,30)=0.004, p=.95]$, interaction $=[F(1,30)=2.28, p=.14])$. 


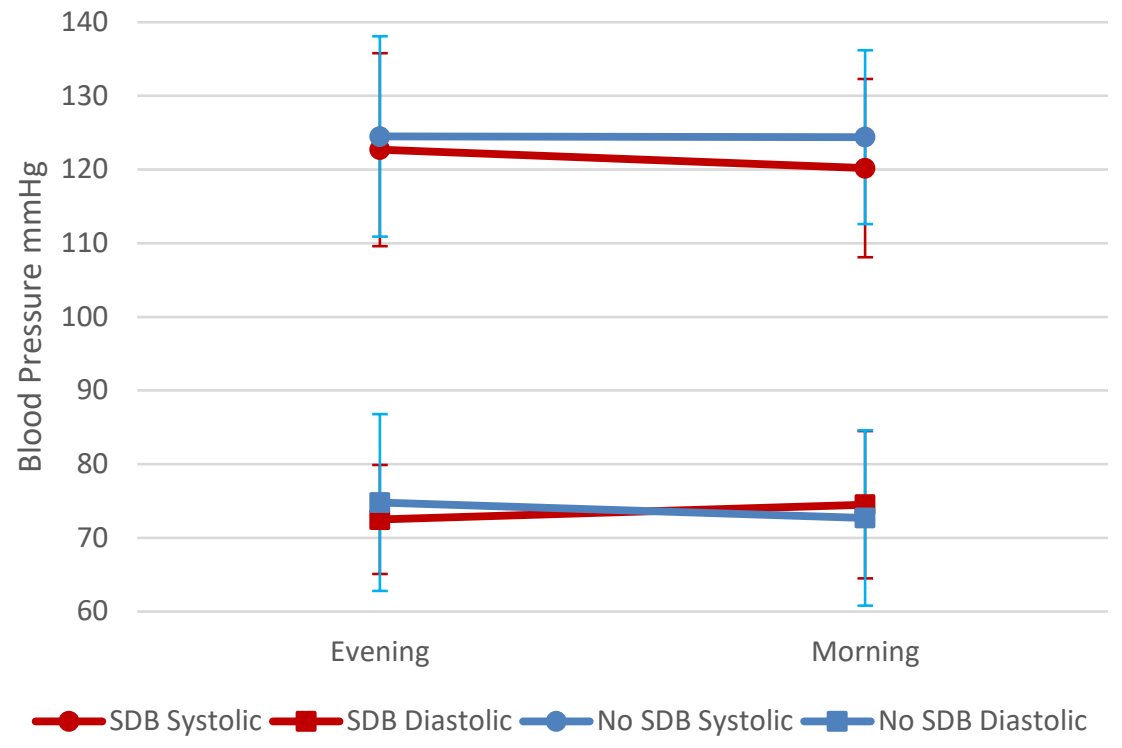

Figure 4.8 Mean \pm SEM overnight blood pressure on the night of the sleep study for the control group, for those with and without $S D B . S D B=$ sleep-disordered breathing.

\subsubsection{Angiogenic Markers of Hypertensive Disease}

The gestational age at which the third trimester sample was taken did not differ based on SDB status within the GH/PE or control group, and there was no difference in the number of days between the third trimester and delivery samples (Table 4.4). The individual results for each participant are shown in Supplementary Figures 4.12 and 4.13 .

As shown in Figure 4.9, the GH/PE and control cohorts showed no difference in ET-1, sFlt-1, sEng or PIGF between the SDB and No SDB group in the third trimester or at delivery. There was also no difference in change per week of gestation within the GH/PE group for those with or without SDB for ET-1, sFIt-1, sEng and PIGF (Table 4.4). Within the control group, there was no difference in the average change per week for ET-1 and PIGF. There was a non-significant trend for the increase in sFIt1 and sEng per week to be higher in the No SDB group compared to the SDB group $(p=.052)$.

A comparison of the angiogenic markers of hypertensive disease for the $\mathrm{GH}, \mathrm{PE}$ and control groups is shown in Supplementary Table 4.8. 
Table 4.4 Change Per Week for Endothelin-1, Soluble Flt-1, Soluble Endoglin and Placental Growth Factor

\begin{tabular}{|c|c|c|c|c|c|c|}
\hline & \multicolumn{2}{|c|}{$\begin{array}{c}\mathrm{GH} / \mathrm{PE} \\
(\mathrm{n}=23)^{*}\end{array}$} & \multicolumn{4}{|c|}{$\begin{array}{l}\text { Controls } \\
(n=24)\end{array}$} \\
\hline & $\begin{array}{c}\text { SDB } \\
(n=13)\end{array}$ & $\begin{array}{l}\text { No SDB } \\
(\mathrm{n}=10)\end{array}$ & $\mathrm{p}$ & $\begin{array}{c}\text { SDB } \\
(n=8)\end{array}$ & $\begin{array}{l}\text { No SDB } \\
(n=16)\end{array}$ & $p$ \\
\hline $\begin{array}{l}\text { Gestation T3 sample } \\
\text { (weeks + days) }\end{array}$ & $35+1(34+1,36+6)$ & $36+1(35+0,37+4)$ & .19 & $32+6(32+0,34+3)$ & $34+1(33+0,34+6)$ & .26 \\
\hline Days between sample & $13.0(8.0,25.0)$ & $8.0(5.0,15.0)$ & .13 & $41.5(25.8,47.8)$ & $37.0(29.5,53.3)$ & .98 \\
\hline ET-1 & $0.11(-0.15,0.31)$ & $0.05(-0.30,0.61)$ & .78 & $0.09(0.02,0.21)$ & $0.05(-0.04,0.14)$ & .29 \\
\hline sFIt-1 & $553.3(96.8,1228.8)$ & $872.6(-379.1,1252.9)$ & .83 & $204.6(151.1,369.2)$ & $459.6(313.0,750.8)$ & .052 \\
\hline sEng & $0.6(-0.8,4.2)$ & $-0.3(-1.7,2.9)$ & .74 & $0.2(-0.2,1.0)$ & $0.8(0.5,1.9)$ & .052 \\
\hline PIGF & $-7.1(-22.7,1.9)$ & $-4.5(-12.3,0.9)$ & .65 & $-10.3(-29.3,-3.4)$ & $-17.9(-35.9,-8.1)$ & .26 \\
\hline
\end{tabular}

Note. Values given as Mdn (IQR). GH = gestational hypertension, PE = preeclampsia, SDB = sleep-disordered breathing, T3= third trimester, ET-1 = endothelin1, sFlt-1 = soluble fms-like tyrosine kinase-1, sEng = soluble endoglin, PIGF = placental growth factor. Three participants on CPAP excluded.

*One PE participant was excluded from analysis due to extreme outlying values ( $z$ scores $>5.3$ ) on all change per week measures except PIGF. 


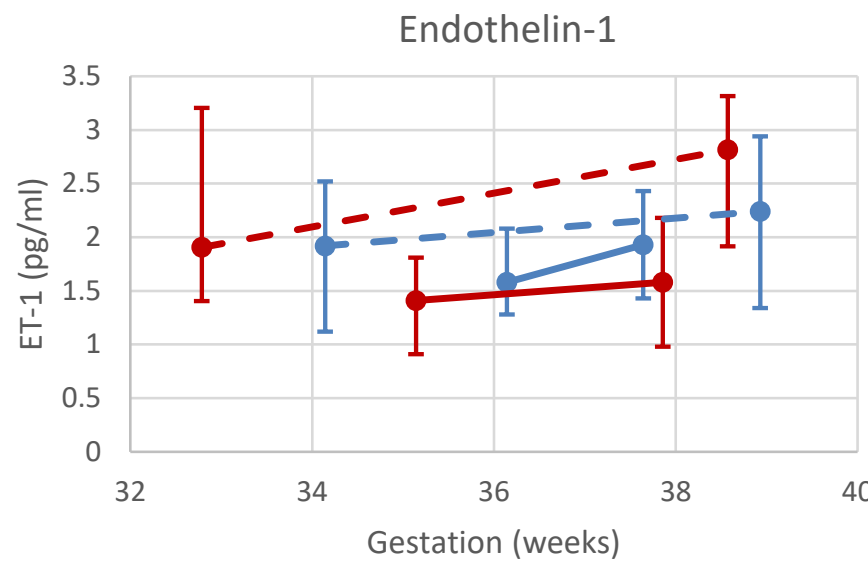

Soluble Endoglin

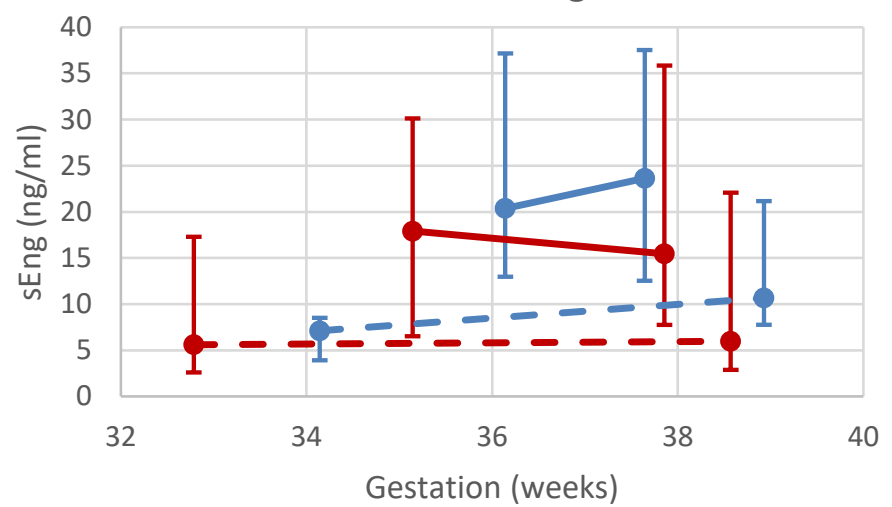

Soluble Flt_1

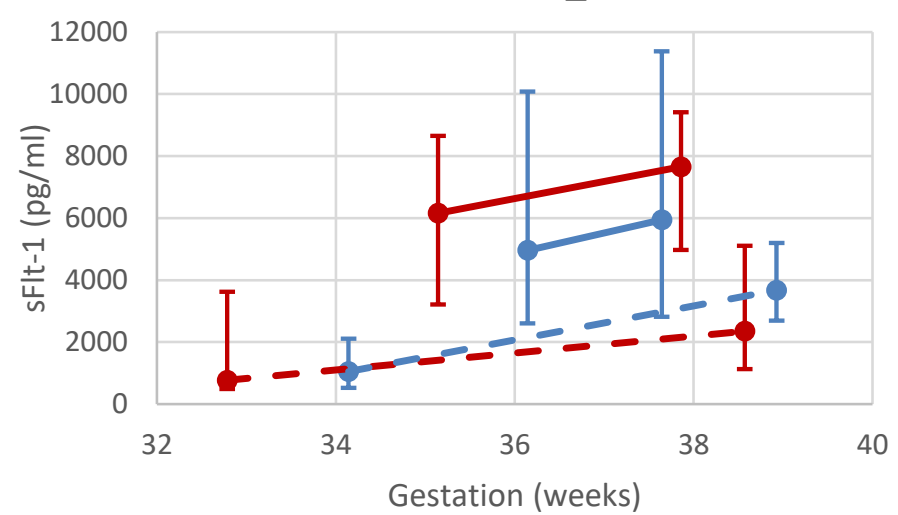

Placental Growth Factor

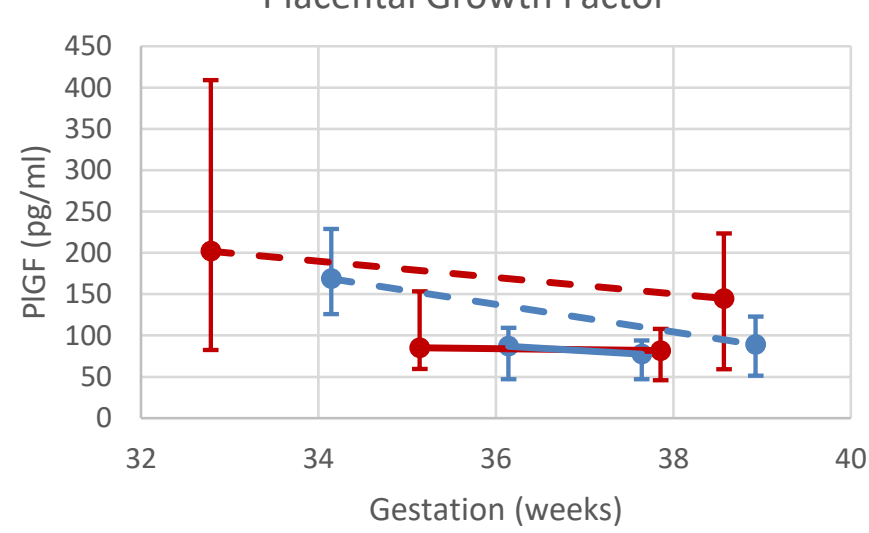

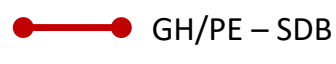

GH/PE - no SDB

$\leadsto$ Control-SDB

$\cdots$ Control-no SDB

Figure 4.9 Median values (with IQR) for endothelin-1 (ET-1), soluble fms-like tyrosine kinase-1 (sFlt-1), soluble endoglin (sEng) and placental growth factor (PIGF) measured in the third trimester and at delivery. The horizontal axis indicates the average gestation at which the third trimester and delivery samples were collected. The difference in rate of change per week between the SDB (RDI $\geq 5)$ and No SDB group in sFIt-1 and sEng within the control group was nearing significance (both $p=.052$ ). $G H=$ gestational hypertension, $P E=$ preeclampsia, $S D B=$ sleep-disordered breathing. 


\subsubsection{Severity of Sleep-Disordered Breathing and its Impact on Hypertensive Disorders of Pregnancy}

In a planned sub-group analysis, altering the threshold for diagnosis of SDB to a higher severity level of $\mathrm{RDI} \geq 15$ did not confer a worse prognosis. In fact, those women with at least moderate SDB and coexisting hypertension in pregnancy appeared to have a better outlook in some aspects of analysis.

At a cut-off of $R D I \geq 15$, those with $P E$ and SDB delivered their babies almost five weeks later than those with PE without SDB ( $p=.005$, Table 4.5$)$, however it should be noted that only 3 participants with $\mathrm{PE}$ had an RDI $\geq 15$ (excluding the participant on CPAP) for this analysis. The average platelet count also tended to be lower in the women without SDB in the GH group $(p=.06)$. On the evening of the sleep study night, systolic BP was significantly lower in those with GH and SDB compared to those with GH without SDB ( $p=.007$, Table 4.6), with a similar trend towards lower systolic BP in the morning $(p=.10)$.

In terms of angiogenic markers of hypertensive disease (Figure 4.10), those with GH/PE and SDB had lower ET-1 levels at delivery $(p=.06)$, and sEng was lower in the third trimester $(p=.052)$ and at delivery $(p=.062)$ at $R D I \geq 15$, compared to those without SDB. Within the control group the same trend continued for the change per week in SFlt-1 to be greater in the No SDB group compared to the SDB group (Table 4.7, $\mathrm{p}=.06$ ). 
Table 4.5 Key Results for Each Group with SDB Defined as an RDI $\geq 15$

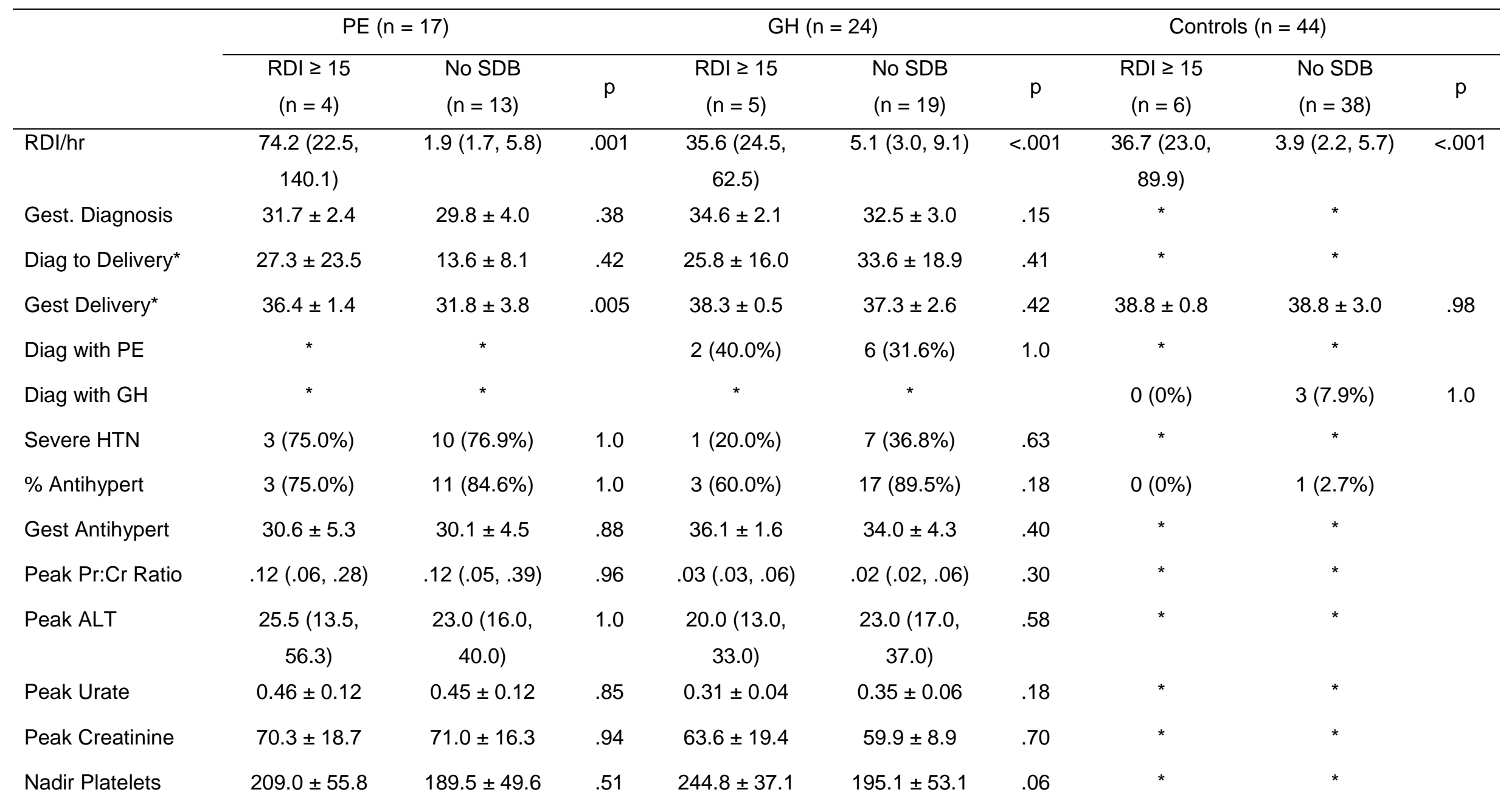

Note. Values given as $\mathrm{M} \pm \mathrm{SD}, \mathrm{Mdn}(\mathrm{IQR})$, or $\mathrm{n}(\%) . \mathrm{SBD}=$ sleep-disordered breathing, $\mathrm{RDI}=$ respiratory disturbance index, $\mathrm{PE}=$ preeclampsia, $\mathrm{GH}=$ gestational hypertension, gest $=$ gestation, diag = diagnosis, $\mathrm{HTN}=$ hypertension, Antihypert $=$ antihypertensive, $\mathrm{Pr}: \mathrm{Cr}=$ protein:creatinine, $\mathrm{ALT}=$ alanine transaminase. ${ }^{*}$ Three CPAP users removed from analysis for these outcomes. 
Table 4.6 Key Blood Pressure Results for Each Group with SDB Defined as an RDI $\geq 15$

\begin{tabular}{|c|c|c|c|c|c|c|c|c|c|}
\hline & \multicolumn{2}{|c|}{$\begin{array}{c}\text { PE } \\
(n=17)\end{array}$} & \multirow[b]{2}{*}{$\mathrm{p}$} & \multicolumn{2}{|c|}{$\begin{array}{c}\mathrm{GH} \\
(\mathrm{n}=24)\end{array}$} & \multicolumn{4}{|c|}{$\begin{array}{l}\text { Controls } \\
(n=39)\end{array}$} \\
\hline & $\begin{array}{c}\mathrm{RDI} \geq 15 \\
(\mathrm{n}=4)\end{array}$ & $\begin{array}{l}\text { No SDB } \\
(n=13)\end{array}$ & & $\begin{array}{c}\mathrm{RDI} \geq 15 \\
(\mathrm{n}=5)\end{array}$ & $\begin{array}{l}\text { No SDB } \\
(n=19)\end{array}$ & $\mathrm{p}$ & $\begin{array}{c}\mathrm{RDI} \geq 15 \\
(\mathrm{n}=6)\end{array}$ & $\begin{array}{l}\text { No SDB } \\
(\mathrm{n}=33)\end{array}$ & $\mathrm{p}$ \\
\hline \multicolumn{10}{|l|}{ Peak Antenatal BP } \\
\hline Systolic mmHg & $161.3 \pm 15.3$ & $166.6 \pm 10.1$ & .42 & $154.8 \pm 4.4$ & $158.4 \pm 11.1$ & .50 & $126.8 \pm 9.3$ & $128.3 \pm 10.1$ & .75 \\
\hline Diastolic mmHg & $103.8 \pm 6.2$ & $102.4 \pm 5.0$ & .66 & $98.8 \pm 6.6$ & $101.7 \pm 6.4$ & .38 & $79.2 \pm 7.6$ & $80.0 \pm 7.4$ & .80 \\
\hline \multicolumn{10}{|l|}{ Peak Postnatal BP } \\
\hline Systolic mmHg & $155.5 \pm 4.9$ & $161.7 \pm 14.4$ & .42 & $148.0 \pm 20.2$ & $159.8 \pm 17.5$ & .21 & $134.0 \pm 18.7$ & $131.8 \pm 13.3$ & .73 \\
\hline Diastolic $\mathrm{mmHg}$ & $99.5 \pm 7.9$ & $97.1 \pm 8.1$ & .61 & $93.6 \pm 11.5$ & $97.8 \pm 8.1$ & .35 & $92.3 \pm 17.4$ & $82.1 \pm 9.5$ & .21 \\
\hline \multicolumn{10}{|l|}{ Overnight BP pm } \\
\hline Systolic mmHg & $136.7 \pm 1.5$ & $132.6 \pm 11.1$ & .23 & $130.4 \pm 2.1$ & $142.3 \pm 15.1$ & .007 & $127.8 \pm 15.6$ & $122.8 \pm 12.7$ & .41 \\
\hline Diastolic $\mathrm{mmHg}$ & $87.3 \pm 16.3$ & $81.0 \pm 10.3$ & .40 & $91.8 \pm 11.7$ & $89.5 \pm 9.0$ & .65 & $75.8 \pm 9.7$ & $73.3 \pm 10.4$ & .48 \\
\hline \multicolumn{10}{|l|}{ Overnight BP am } \\
\hline Systolic mmHg & $135.7 \pm 6.7$ & $135.5 \pm 13.6$ & .99 & $127.2 \pm 9.7$ & $140.4 \pm 16.0$ & .10 & $125.8 \pm 14.1$ & $121.8 \pm 11.5$ & .47 \\
\hline Diastolic $\mathrm{mmHg}$ & $86.7 \pm 9.7$ & $83.3 \pm 7.8$ & .53 & $85.8 \pm 7.0$ & $89.6 \pm 10.7$ & .47 & $78.7 \pm 12.2$ & $72.3 \pm 10.6$ & .20 \\
\hline \multicolumn{10}{|l|}{ ON BP change } \\
\hline Systolic mmHg & $-1.0 \pm 7.2$ & $2.9 \pm 11.5$ & .59 & $-3.2 \pm 8.4$ & $-1.8 \pm 11.5$ & .80 & $-2.0 \pm 7.0$ & $-0.9 \pm 7.9$ & .76 \\
\hline Diastolic $\mathrm{mmHg}$ & $-0.7 \pm 8.1$ & $2.3 \pm 10.5$ & .66 & $-6.0 \pm 13.9$ & $0.1 \pm 7.2$ & .21 & $2.8 \pm 7.2$ & $-1.0 \pm 7.9$ & .28 \\
\hline
\end{tabular}

Note. Values given as $\mathrm{M} \pm \mathrm{SD} . \mathrm{SBD}=$ sleep-disordered breathing, $\mathrm{RDI}=$ respiratory disturbance index, $\mathrm{PE}=$ preeclampsia, $\mathrm{GH}=$ gestational hypertension, $\mathrm{BP}$

$=$ blood pressure, $\mathrm{ON}=$ overnight. 
Endothelin-1

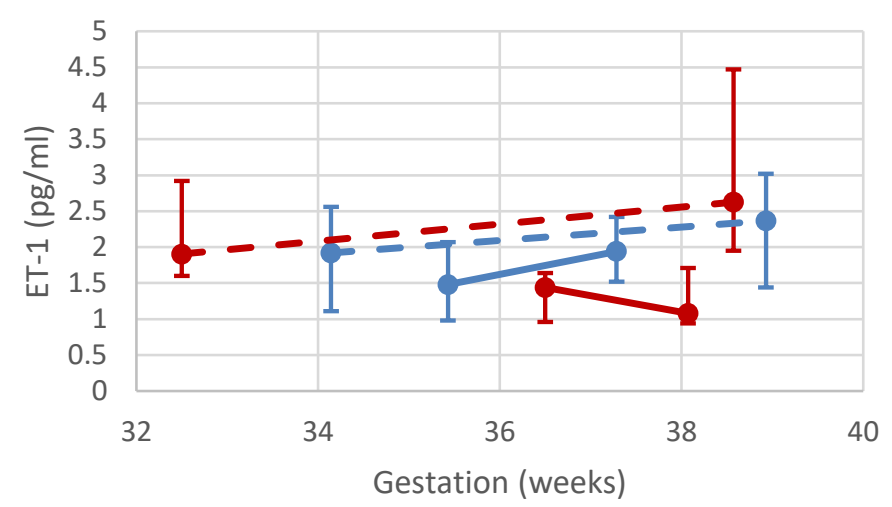

Soluble Endoglin

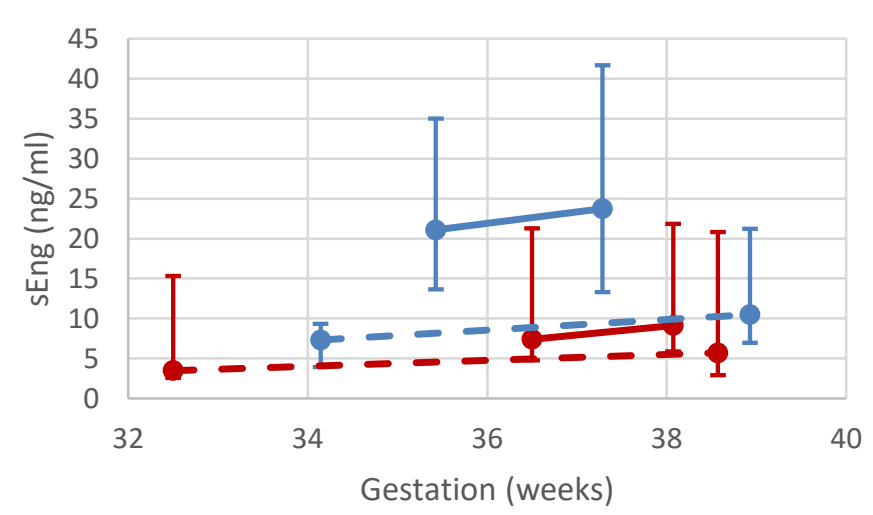

Soluble Flt_1

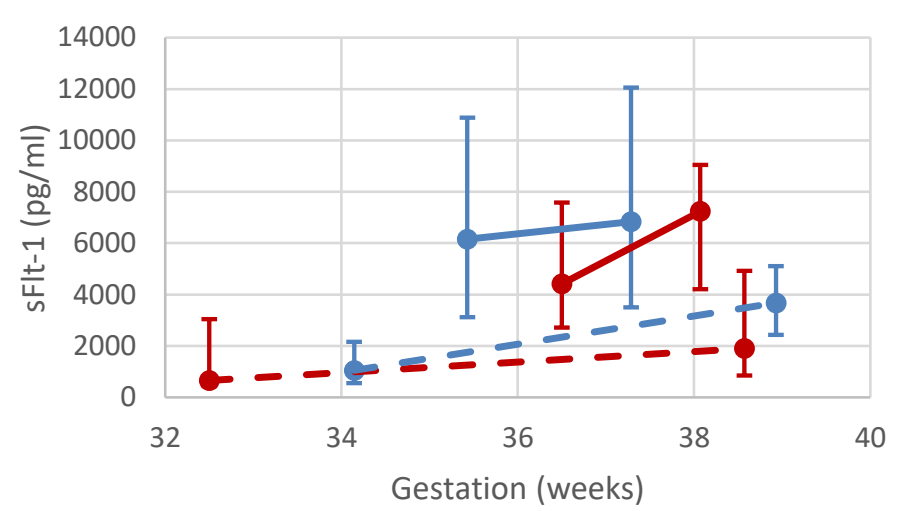

\section{Placental Growth Factor}

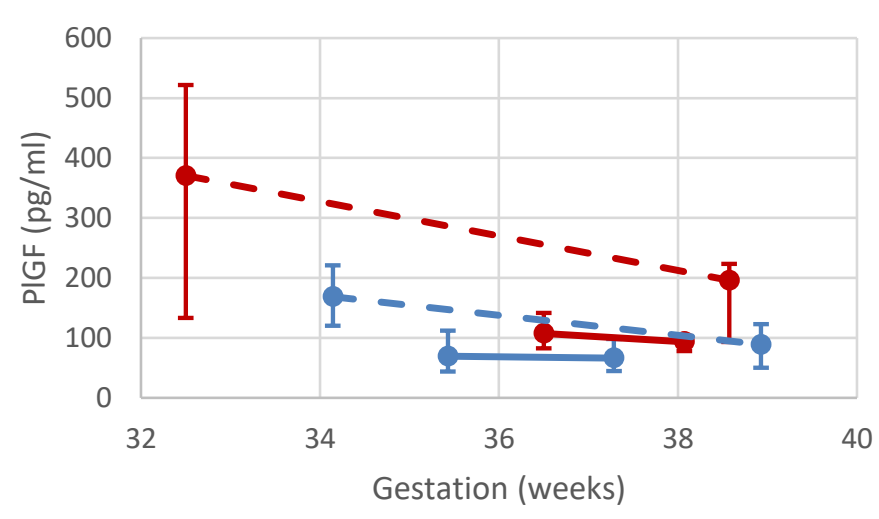

$\longrightarrow \mathrm{GH} / \mathrm{PE}-\mathrm{SDB} \mathrm{RDI} \geq 15$ GH/PE - no SDB

$\cdots$ Control-SDB RDI $\geq 15$

$-\infty$ Control - no SDB

Figure 4.10 Median values (with IQR) for endothelin-1 (ET-1), soluble fms-like tyrosine kinase-1 (sFlt-1), soluble endoglin (sEng) and placental growth factor (PIGF) measured in the third trimester and at delivery, with SDB defined as RDI $\geq 15$. The horizontal axis indicates the average gestation at which the third trimester and delivery samples were collected. Within the GH/PE group, ET-1 was lower at delivery in the SDB group ( $p=.06)$, and $s E n g$ was lower in the third trimester $(p=.052)$ and at delivery $(p=.06)$ in the SDB group. In the control group, there was a trend towards a greater increase per week in sFlt-1 for those without $S D B(p=.06)$. $G H=$ gestational hypertension, $P E=$ preeclampsia, $S D B=$ sleep-disordered breathing, $R D I=$ respiratory disturbance index . 
Table 4.7 SDB defined as RDI $\geq 15$ - Change Per Week for Endothelin-1, Soluble Flt-1, Soluble Endoglin and Placental Growth Factor

\begin{tabular}{|c|c|c|c|c|c|c|}
\hline & \multicolumn{2}{|c|}{$\mathrm{GH} / \mathrm{PE}$} & \multicolumn{4}{|c|}{ Controls } \\
\hline & $\begin{array}{c}\mathrm{RDI} \geq 15 \\
(\mathrm{n}=6)\end{array}$ & $\begin{array}{l}\text { No SDB } \\
(n=17)\end{array}$ & $\mathrm{p}$ & $\begin{array}{c}\mathrm{RDI} \geq 15 \\
(\mathrm{n}=4)\end{array}$ & $\begin{array}{l}\text { No SDB } \\
(n=20)\end{array}$ & $\mathrm{p}$ \\
\hline $\mathrm{RDI} / \mathrm{hr}$ & $37.7(25.3,59.2)$ & $3.5(1.9,7.5)$ & $<.001$ & $27.3(20.3,39.9)$ & $3.8(2.1,4.6)$ & $<.001$ \\
\hline ET-1 & $-0.04(-0.21,0.14)$ & $0.20(-0.13,0.66)$ & .29 & $0.09(0.04,0.29)$ & $0.05(-0.04,0.14)$ & .35 \\
\hline sFIt-1 & $679.2(292.8,1790.3)$ & $553.3(-181.5,1184.6)$ & .56 & $153.4(57.6,323.4)$ & $394.2(275.0,717.1)$ & .06 \\
\hline sEng & $0.7(-0.1,3.8)$ & $-0.3(-1.1,4.2)$ & .56 & $0.3(0.0,1.0)$ & $0.7(0.2,1.9)$ & .31 \\
\hline PIGF & $-14.9(-23.0,4.6)$ & $-4.1(-14.4,0.0)$ & .56 & $-22.6(-38.5,-4.7)$ & $-13.7(-32.1,-6.1)$ & .85 \\
\hline
\end{tabular}

Note. Values given as Mdn (IQR). SDB = sleep-disordered breathing, RDI = respiratory disturbance index, GH = gestational hypertension, $\mathrm{PE}=$ preeclampsia, $\mathrm{ET}-1$ = endothelin-1, sFlt-1 = soluble fms-like tyrosine kinase 1, sEng = soluble endoglin, PIGF = placental growth factor. Three participants on CPAP excluded. 


\subsection{Discussion}

\subsubsection{Diagnosis and Severity of Hypertensive Disease}

The overall aim of this chapter was to determine whether the presence of SDB influenced the course and severity of hypertensive disease in pregnancy. We hypothesised that women with $\mathrm{PE}$ or $\mathrm{GH}$, in combination with SDB would have more malignant hypertensive disease. Women with SDB were not diagnosed with either PE or GH earlier in pregnancy than those without SDB. Of particular clinical concern was whether SDB would alter the clinical course of GH or PE and require delivery at an earlier gestation due to complications. This was not the case for either the PE or GH group - SDB had no significant effect on the maintenance of pregnancy after diagnosis, or the gestation at delivery of the baby.

Most hypertensive women required medication and in fact, the use of multiple anti-hypertensives for BP control was required more often in women with PE who did not have SDB. Presence of SDB did not worsen indicators of renal or hepatic functioning typically associated with PE. Within the GH group, the same proportion (33\%) of those with and without SDB went on to develop PE.

We also hypothesised that normotensive women with SDB would be more likely to develop hypertension in the remainder of their pregnancy. Three out of 44 control women developed $\mathrm{GH}$, and only one of them had SDB. No normotensive women went on to develop PE. Again, the presence of SDB did not impact on the gestation of delivery of the baby.

Our results however do raise the possibility that paradoxically, hypertensive women with more severe SDB have a better outlook. Specifically, women with PE and moderate to severe SDB had pregnancies prolonged by around five weeks of gestation, and although not significant, had twice as long between diagnosis of PE and delivery compared to those with an RDI $<15$. Women with HDP and SDB also had lower levels of HDP markers ET-1 and sEng at delivery. It is important to consider the potential that HDP linked to SDB has a more benign course than that associated with primary placental ischemia, which is something we can only speculate here but is worth considering for future research.

\subsubsection{Preeclampsia Development and the Potential Impact of Sleep-Disordered Breathing}

There is substantial cross over between the pathophysiological mechanisms associated with SDB and the pathogenesis of PE. The development of PE is hypothesized to be in two distinct phases, both of which could hypothetically be augmented by SDB. The first stage is reduced placental perfusion caused by errors in vascular remodeling, followed by placental ischemia/hypoxia which lead to the second symptomatic phase hallmarked by widespread maternal endothelial dysfunction. ${ }^{324,325}$

At the initial stage of PE development, the placentation process is aberrant. The invasion of cytotrophoblasts of fetal origin into the maternal uterine wall is incomplete, with cytotrophoblast cells 
present in only the superficial layers of the decidua. ${ }^{326}$ The spiral arteries fail to be invaded or remodeled, resulting in constricted, high-resistance vessels. As a result of this failure to remodel, insufficient blood flow to the uteroplacental unit results and the placenta becomes hypoxic. ${ }^{324}$

The cause of this abnormal implantation and vascular remodeling is unknown, but proposed factors include hypoxia and oxidative stress, ${ }^{325}$ as well as Angiotensin II Type 1 receptor activating antibodies (AT1-AA), immunologic and genetic factors. ${ }^{136}$ Inflammatory cytokines such as TNF- $\alpha$ and IL-6 are also thought to interfere with trophoblast implantation ${ }^{182,183}$; plasma concentrations of these cytokines have been found to be significantly increased in both early- and late-onset PE compared to healthy women, and are markedly more elevated when PE is severe..$^{177,179}$

Hypoxia associated with SDB may contribute to abnormal placental development, because the failure of cytotrophoblasts to fully invade and switch to adhesion molecules can also be reproduced in vitro when cytotrophoblasts are cultured under hypoxic conditions. ${ }^{327}$ In animal models, intermittent hypoxia/reoxygenation injury leads to oxidative stress and reductions in antioxidant levels, ${ }^{173}$ and there is accumulating evidence from human studies that untreated SDB causes oxidative stress. ${ }^{328}$ In PE, oxidative stress is demonstrable both in the maternal circulation and in the placenta - preeclamptic placentas produce greater quantities of superoxide and have less antioxidant capacity than normal placentas. ${ }^{160}$ Patients with SDB may have significantly increased levels of inflammatory markers such as TNF- $\alpha$, CRP and IL-6, ${ }^{54,56,175,176}$ which decrease back to normal levels following treatment. ${ }^{56}$ At this early stage of pregnancy, it could be hypothesised that women with pre-existing SDB experiencing nocturnal hypoxia with subsequent oxidative stress and inflammation could be on an early pathway to developing PE.

During the second phase of PE development, the under-perfused placenta releases factors into the circulation, which then trigger maternal endothelial dysfunction. ${ }^{325}$ These factors include markers of oxidative stress, inflammatory cytokines, ET-1 and anti-angiogenic factors, ${ }^{325}$ which lead to the maternal syndrome of PE characterized by hypertension, renal insufficiency and proteinuria, liver dysfunction and cerebral edema. ${ }^{136}$

At this second stage of PE evolvement, the pathological sequalae of SDB could heighten the maternal endothelial damage seen in PE, increasing susceptibility of the vasculature to the effects of circulating anti-angiogenetic factors, ${ }^{187}$ resulting in worsening tissue damage. It has also been suggested that women with moderate levels of SDB are more vulnerable to endothelial dysfunction than men. ${ }^{329}$ Intermittent hypoxia and reoxygenation with the additional production of oxygen radicals has been proposed to be more harmful than sustained hypoxia in terms of its contribution to oxidative stress. ${ }^{172}$ Compounding the damage to the already ischemic placenta, intermittent hypoxia from SDB could further induce the release of anti-angiogenic factors. 
A recent study which is the largest of its kind found robust evidence for a relationship between SDB measured in early and mid-pregnancy and HDP. ${ }^{123}$ They specifically found that $92 \%$ of the hypertension diagnoses were made more than 2 weeks after the midpregnancy sleep study performed at a median of 28 weeks gestation. This supports earlier studies finding that SDB was independently associated with the development of PE in obese pregnant women, ${ }^{124}$ and with $\mathrm{PE}$ and $\mathrm{GH}$ in non-obese pregnant women. ${ }^{280}$ No studies have specifically looked into changes in the diagnostic features of gestational hypertensive disorders associated with SDB, such as severity of hypertension, early onset of disease, antihypertensive use, biochemical and haematological markers of disease severity or prolongation of pregnancy following diagnosis of disease. Rather, just two studies have suggested that treatment of mild SDB in preeclamptic women results in reductions in nocturnal BP and minimisation of cardiac output reductions. ${ }^{169,270}$

Despite the plausible biological arguments for how SDB could have a detrimental effect on the diagnosis and severity of hypertensive disease during pregnancy and the aforementioned literature, our results did not support this. We found that women who had SDB during their pregnancy did not experience worse outcomes in relation to their co-existing $\mathrm{PE}$ or $\mathrm{GH}$, and normotensive women were no more likely to develop a hypertensive disorder. In support of our negative results, three studies have found that SDB did not impact on the incidence of hypertensive disease during pregnancy. 7,262,263

The most pertinent possibilities for a lack of association between SDB and development of HDP relate to the phenotype of both SDB and PE featured in our study. As discussed more thoroughly in Section 4.4.3 - Severity of SDB, the overall degree of SDB diagnosed in our sample was predominantly mild, with a low number of hypoxaemic episodes experienced by the mothers. Many studies focus solely on PE, whereas the combining of both GH and PE into an HDP group for Chapter 3 means our sample size within each of the hypertensive groups has been reduced, potentially affecting the power required to successfully demonstrate any associations. Additionally, we had a range of PE severity featuring in our study, with both a maternal or fetal pathology dominating the diagnoses. Whilst some studies concentrated on women with severe PE or those admitted and potentially requiring imminent delivery, $169,259,270$ we had participants with both mild and severe PE in our study who were either in- or out-patients, with pregnancy continuing between one day to six weeks post diagnosis of PE.

An alternate hypothesis on the cause of $\mathrm{PE}$ and a potential reason why our results were negative is the immune theory of PE. Epidemiological studies support the concept of maternal-fetal (paternal) immune maladaptation being centrally implicated in the causation of $\mathrm{PE},{ }^{330,331}$ rather than ischaemia-reperfusion leading to oxidative stress and vascular disease. Deposition of semen into the female genital tract provokes a cascade of cellular and molecular events that resemble a classic inflammatory response, ultimately leading to superficial placentation. Major support for this hypothesis comes from studies showing that main risk factors of PE are limited sperm exposure with the same partner before conception, ${ }^{332}$ change in paternity, ${ }^{331,333}$ and pregnancies after donor insemination and oocyte donation. 334,335 


\subsubsection{Placental Factors}

Circulating anti-angiogenic proteins released by the ischemic placenta have been implicated as the mediators of many of the maternal features of PE. ${ }^{136}$ PIGF, part of the family of vascular endothelial growth factors (VEGF), is a pro-angiogenic factor that promotes proliferation and survival of endothelial cells and induced vascular permeability. ${ }^{336,337}$ In PE, excess placental secretion of sFlt-1 and sEng causes endothelial dysfunction by inhibiting PIGF and transforming growth factor- $\beta 1$ (TGF $\beta 1$ ) signalling in the vasculature, which is required to maintain endothelial health in several tissues including the kidney and perhaps the placenta. ${ }^{136}$

\subsection{Anti-Angiogenic Factors - Soluble Flt-1 and Soluble Endoglin}

SFIt-1 and sEng are placentally derived anti-angiogenic factors. They are among the most extensively researched anti-angiogenic factors in PE and were therefore a focus in our study. Epidemiological studies have revealed that in PE, circulating levels of sFlt-1 begin to rise at least 5 weeks before the onset of clinical disease and remain elevated compared with healthy pregnant women. ${ }^{159,188,338}$ SFIt-1 is increased further in patients who have early onset PE. ${ }^{188}$ SEng levels in women with normal pregnancies are stable until approximately week 33 of pregnancy, when they rise, peaking at delivery. However, in women with preterm PE, levels of sEng begin to rise by 20 weeks gestation and rise more steeply after 33 weeks. ${ }^{190}$ As expected, our results showed that sFlt-1 and sEng were substantially higher in the GH/PE group compared to the healthy controls, particularly in the PE participants. However, the rate of increase over time did not differ across groups.

The effect of placental hypoxia on sFlt-1 has been investigated; in animal models, surgical uterine ischemia has been shown to induce elevated circulating sFlt-1 levels in addition to the PE-like syndrome. ${ }^{339,340}$ The impact of hypoxaemia on placental anti-angiogenic markers has been examined using SDB as the hypoxic model. In humans, both sFlt-1 and sEng are increased in the circulation of patients with OSA. ${ }^{196,197}$ In contrast, our results showed no differences in the levels of SFIt-1 or sEng during pregnancy between those with or without SDB, and within the hypertensive group the change in sFIt-1 and sEng over time was not statistically different. Surprisingly, within the controls it was those without SDB who had a greater increase in both sFlt-1 and sEng between the third trimester and delivery, rather than those with SDB.

\subsection{Pro-Angiogenic Factor - Placental Growth Factor}

PIGF binds to the Flt-1 receptor with high affinity, and is suggested to play a role in angiogenesis in pathological settings. ${ }^{341}$ PIGF levels increase in high amounts in the second trimester, peak during weeks 29 to 32 , and decline thereafter. ${ }^{136}$ PIGF is used clinically as a marker of PE. Levels of free PIGF are depressed in women with PE, with the degree of suppression of serum/urinary PIGF levels correlated with severity of PE. ${ }^{342}$ Similarly, we found that the hypertensive groups, particularly the PE group, had significantly lower levels of PIGF during the third trimester compared to normotensive women. 
The impact of hypoxia and SDB on PIGF has received little attention. One study has shown that PIGF levels were significantly elevated in acute coronary syndrome patients with OSA compared to those without OSA. ${ }^{343}$ Hypoxia associated with high altitude does not alter levels of circulating PIGF in normal or PE pregnancies. ${ }^{344}$ Similarly, we found that the absolute values of PIGF in the third trimester and at delivery, and the decrease in PIGF during this time was not affected by SDB status.

\subsection{Endothelin-1}

ET-1 is a potent vasoconstrictive peptide with BP-elevating properties, produced by endothelial cells. ${ }^{173}$ The release of anti-angiogenic factors from the ischemic placenta causes endothelial injury, which then induces hypertension through the production of ET-1. ${ }^{324,345}$ Circulating levels of ET-1 have been found to be elevated in preeclamptic compared to normal pregnancy. ${ }^{315,317}$ Our results were unexpected, in that ET-1 was actually higher in the normotensive group compared to those with hypertensive disease during pregnancy, but increased at a similar rate from third trimester to delivery. Also, ET-1 was not elevated in the PE compared to GH group.

Several studies have demonstrated enhanced levels of circulating ET-1 in OSA, and its decrease after treatment with CPAP, 313,314 however one contrasting study did report that ET-1 levels were not elevated in OSA. ${ }^{346}$ Such conflicting findings may reflect the actual levels of hypoxaemia patients experienced, or individual differences in the response to the hypoxic stimulus. ${ }^{347}$ In our study, levels of ET-1 were not influenced by SDB in either the normotensive or hypertensive pregnancy group.

\subsection{Placental Factors - Summary}

Few studies, and mostly case reports, have looked at angiogenic biomarkers of PE amongst those with comorbid SDB. We presented a woman diagnosed with early-onset $P E$ at 30 weeks gestation who was confirmed to have very severe SDB $(\mathrm{AHI} / \mathrm{hr}=149) .{ }^{158}$ Treatment with CPAP in this case resulted in a reduction of the circulating anti-angiogenic factors sFlt-1 and sEng, and stabilisation of ET-1, which paralleled improvement in both clinical and biochemical measures of PE. A published abstract in 2012 described elevated sFlt-1 levels associated with untreated SDB in two pregnant women with hypertensive disease compared to a hypertensive woman treated with CPAP and a healthy control. ${ }^{348}$ A case study from this group has recently been published regarding CPAP treatment of mild SDB in a pregnant woman with chronic hypertension. ${ }^{349}$ They found that sFlt-1 concentrations rose only $16 \%$ across pregnancy from a low baseline and the patient did not develop PE or require an escalation in her antihypertensives. The only case control study in this area was performed by Bourjeily and colleagues, ${ }^{198}$ which showed that circulating placenta-secreted glycoproteins and markers of angiogenesis (PAPP-A and SFIt-1/PIGF ratio) are altered in pregnant women with OSA.

In a related vein, one other study has found a weak negative trend towards a correlation between endothelial function and RDI. ${ }^{184}$ Women who experience sleep disturbances, but not specifically SDB, were likely to have an increase in inflammation in terms of IL- 6 but not TNF- $\alpha,{ }^{350}$ whereas no 
improvement of inflammatory markers were seen following a single night of CPAP for mild SDB in pregnancy. ${ }^{274}$ We were unable to confirm any adverse impact of SDB on the anti-angiogenic biomarkers of PE.

Although we determined PE and GH to be different disease processes, we needed to analyse the angiogenic markers together due to lack of blood samples obtained in the PE group. Vascular biology studies have suggested that there is a contrasting pathophysiology between PE and GH, for example maternal blood levels of the anti-angiogenic markers of sFlt-1 and sEng were elevated only in women with PE, ${ }^{321}$ and levels of endothelial microparticles (associated with endothelial cell damage) were found to be significantly higher in women with PE but not GH. ${ }^{320}$ As we showed in the supplementary results, the PE and GH results for sFlt-1, sEng and PIGF did show some significant differences. Our results may have been influenced by the presence of fewer PE compared to GH participants within our analysis, as well as a more benign PE phenotype given those with maternal blood sampled delivered at least one to five weeks post diagnosis. However, our outcome of interest related to the negative impact that SDB, not severity of hypertensive disease, may have on angiogenic markers and our results showed no suggestion of significant differences.

Timing of collection of blood samples in the third trimester and delivery was quite variable. As control participants were usually recruited earlier in pregnancy there was more flexibility in when the third trimester sample could be taken, and GH and PE participants typically delivered their babies sooner, resulting in a shorter time lapse between the $\mathrm{GH}$ and PE samples compared to the controls. Given the unavoidable differences in gestational age at recruitment and delivery, we calculated a change per week assessment, and there was no difference in the time elapsed between samples for those with and without SDB.

\subsubsection{Blood Pressure Control during Pregnancy}

\subsubsection{Hypertension in Pregnancy and the Impact of Sleep-Disordered Breathing}

In normal pregnancy, BP initially decreases until around 18-20 weeks gestation and then rises back to baseline levels until delivery. A large longitudinal study of over 13,000 pregnant women has shown that women with HDP have greater increases in BP from 18 weeks onwards compared to normotensive women $^{351}$ and in the third trimester BP increases were distinctly greater in women who developed PE compared to those who developed GH. ${ }^{351,352}$ Our results showed an increase in SBP and DBP from around 20 weeks gestation until delivery for our gestational hypertensive and normotensive groups. In contrast to the Macdonald et al. ${ }^{351}$ and Gaillard et al. ${ }^{352}$ studies, the rise in BP in our PE group was not significant, and was no more substantial than that amongst the GH group.

Most importantly and in contrast to our hypotheses, the presence of SDB did not contribute to higher BP measurements during hypertensive pregnancies - in fact the women with SDB in the GH group had lower SBP and DBP on average across pregnancy. Similarly, overall peak SBP and DBP measured in 
pregnancy, postpartum and in the intrapartum period was not increased in women with SDB compared to those without.

Our results are surprising given past literature in the non-pregnant population. As mentioned, patients with SDB spend their sleep periods in a state of intermittent hypoxia and a cyclic pattern of recurrent surges of vasoconstriction. Increased sympathetic tone (as a result of SDB in the sleep period) exerts systemic changes that promote persistence of increased $\mathrm{BP}^{353}$; sympathetic activity has been shown to be increased during the daytime in patients with OSA, even in awake, normoxic conditions. ${ }^{308,354} \mathrm{It}$ is plausible that increased sympathetic activity due to SDB events could be a mechanism for vasoconstriction in some pregnant women ${ }^{236}$ contributing to the development of $\mathrm{GH}$ or PE, however our data did not support this.

Repetitive hypoxic stress can alter sympathetic chemoreflex function in patients with OSA, contributing to increased BP during the daytime. ${ }^{355}$ Patients with OSA may also develop changes in their autonomic regulation of BP consistent with adaptation of the baroreceptors to higher BP set points. ${ }^{356-358}$ In addition, sympathetic activation-mediated vasoconstriction may induce long-lasting structural changes in resistance vessels that contribute to the persistence of hypertension, with animal models of intermittent hypoxia showing early structural and functional vascular changes, ${ }^{359,360}$ along with impaired vasodilator response to hypoxia. ${ }^{361} \mathrm{An}$ increase in vascular resistance is a key characteristic of PE. ${ }^{3}$

A number of studies have found a relationship between SDB and a diagnosis of hypertension in the non-pregnant population. ${ }^{362-364}$ In particular, prevalence rates of hypertension have been linearly related to severity of SDB, ${ }^{365}$ although even those with mild SDB (AHI $\left.\geq 5\right)$ have been shown to have twice the odds of a hypertension diagnosis four years following identification of SDB. ${ }^{65}$ Conversely, two longitudinal studies have found that the odds of developing hypertension 5+ years after diagnosis of SDB increased with a dose-response effect to severity of SDB, however this relationship was attenuated and no longer significant after adjustment for BMI. ${ }^{366,367}$ Attempts to explain the discrepancies between these studies come down to differences in population characteristics, especially in terms of sex, age and BMI, and the differences in diagnostic procedures used (polysomnography vs polygraphy). ${ }^{368}$

Only one study has looked at longitudinal BP control across pregnancy, by comparing the addition of nasal CPAP treatment to standard prenatal care in a small group of women with pre-existing hypertension and snoring but who did not have SDB. The CPAP group showed a progressive decrease in BP and anti-hypertensive medication dosage from the sixth month of pregnancy compared to the control group, implying that correction of even mild levels of SDB may have a positive impact on BP control. 273

Why did we find no evidence to suggest that SDB has a detrimental effect on BP control across pregnancy? One key factor could relate to pregnancy being a transient condition of only nine months. Evidence has suggested that pregnancy can induce SDB, ${ }^{125,369}$ and that it can become worse across 
pregnancy. ${ }^{7,122}$ Development of SDB can depend on other temporary factors such as gestational weight gain and decreased upper airway size.7,92 Most cross-sectional or longitudinal studies in the nonpregnant population could assume that for the most part, participants diagnosed with SDB have had the condition for a reasonable length of time, particularly as SDB is often unrecognised. ${ }^{370}$ Also, large longitudinal studies that have followed up participants at least four years post SDB diagnosis $65,366,371$ allow time for the repetitive apnoea/hypoxia/reoxygenation cycle to evoke changes in the autonomic regulation of BP. We need to consider that the pathophysiology of pregnancy versus non-pregnancy hypertension are likely different. The causes behind major changes in vascular resistance (a key characteristic of PE) that occur in hypertension in the general population are less relevant in a short insult such as pregnancy. If SDB results in chronic cardiovascular changes, it is unlikely that the transient respiratory changes in pregnancy would have been present long enough to result in downstream physiological effects.

The demographics of our sample were also dissimilar to the many studies in this area. We had a group of relatively young, otherwise healthy, females, whereas many studies focus on older populations, ${ }^{65,365,366,372,373}$ often predominantly male. ${ }^{374-376}$ In fact, Hedner et al..$^{377}$ noted an independent association between OSA and hypertension in men but not in women. Many studies also find significant results for those with severe levels of SDB with substantial oxygen desaturations ${ }^{363,376}$ and animal models of baroreflex and chemoreflex controls of sympathetic activity following apnoea tend to replicate severely hypoxic conditions. ${ }^{378-380}$ In comparison, we used a low threshold for diagnosis of SDB, our sample were typically on the mild end of the SDB scale, and severe hypoxia was rare.

Our study design allowed for good control of obesity as a confounder, given our original BMI matched design. Furthermore, we made sure to take obesity into account in each analyses, considering obesity is a risk factor for increased BP during pregnancy and PE, ${ }^{266,381}$ as well as SDB. ${ }^{264}$ The exact mechanisms by which obesity is associated with PE are not understood, however possible explanations include enhanced cytokine-mediated oxidative stress, amplified sympathetic activity and increased inflammation. ${ }^{3}$ It was surprising that BMI was not related to BP control within either hypertensive pregnancy group - perhaps our consistent adjustment for obesity is another reason why our results are discrepant to some of those outside of pregnancy.

Lastly, we have to allow for the possibility of some degree of measurement error in the longitudinal BP recordings. BP measurements at each antenatal appointment were taken by whoever the midwife or obstetrician was at the time and recorded in the participants file. We made the assumption that all of the health professionals performed these measurements in a standardised manner, but we have no way of guaranteeing this.

\subsubsection{Nocturnal Blood Pressure and Sleep-Disordered Breathing}

We hypothesised that women with SDB would have heightened nocturnal BP as measured on the night of the sleep study, as a result of increased sympathetic tone with surges in BP associated with 
obstructive respiratory events. The circadian rhythm of BP during pregnancy is essentially similar to the non-pregnant state. BP reaches a maximum by late evening, with a minimum typically occurring in the early hours of the morning before increasing again during waking hours. ${ }^{382}$ Patients with $\mathrm{GH}$ and mild PE may experience a less pronounced nocturnal decrease, whereas those with severe PE may display a reversed circadian rhythm with no decrease or an increase in nocturnal BP. ${ }^{382,383}$

In patients who have SDB with discrete respiratory events, each obstructive apnoea results in an episode of hypoxia followed by reoxygenation when resumption of breathing occurs. Each hypoxic episode stimulates the carotid chemoreceptors, ${ }^{378,380}$ resulting in sympathetic nerve activation. ${ }^{378}$ On resumption of breathing, cardiac output increases which coincides with the constricted peripheral vasculature, and sympathetic nerve activity is further enhanced by arousal. All of this results in marked increases in arterial BP (See Figure 4.11 for illustration). ${ }^{384,385}$ In preeclamptic pregnancy, Edwards et al. ${ }^{269}$ found that acute beat-to-beat BP responses to obstructive respiratory events during sleep resulted in substantial rises of $38 \mathrm{mmHg}$ systolic and $25 \mathrm{mmHg}$ diastolic BP on average, significantly greater than in normotensive pregnancy. Cumulative increases in BP throughout the night during sleep have also been demonstrated in PE, ${ }^{270}$ with marked increases from the start to the end of the night of $33 \mathrm{mmHg}$ systolic and $26 \mathrm{mmHg}$ diastolic BP on average. Although these women did not have discrete respiratory events with oxygen desaturations, treatment with CPAP was able to ameliorate the rise in BP overnight, hence the authors' conclusion that upper airway flow limitation in $\mathrm{PE}$ is associated with increments in BP.

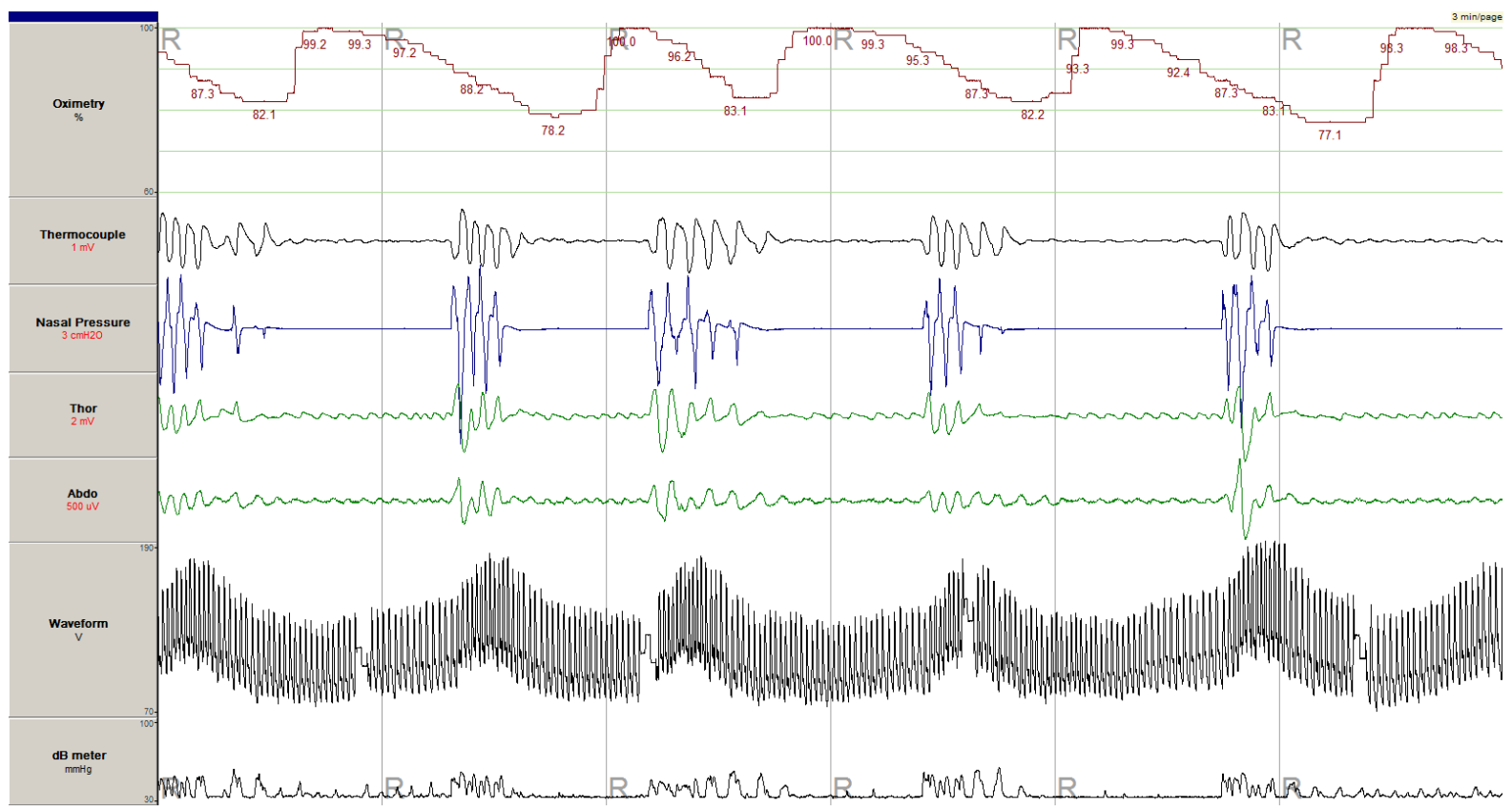

Figure 4.11 Polysomnogram (PSG) showing BP response to obstructive respiratory events with oxygen desaturations to a nadir of $77 \%$, during REM sleep in one of our participants with $G H$ at $36+5$ weeks of gestation. A small number of participants in our study had overnight beat-tobeat finger arterial BP monitoring with the Portapres (Finapres Medical Systems, 
Netherlands) however the sample was too small for meaningful analysis and was excluded from the thesis. Beat-to-beat BP is shown in the 'Waveform' signal ranging from $70-$ $190 \mathrm{mmHg}$, with the peaks representing systolic and the troughs representing diastolic BP. Oximetry $=$ arterial oxygen saturation, Thor $=$ thoracic respiratory effort, Abdo $=$ abdominal respiratory effort, $d B$ meter $=$ snoring.

A number of authors have documented a consistent relationship between SDB and BP in the general population. Studies including those with thousands of participants found that $\mathrm{AHI}$ was linearly associated with 24 hour BP values, even after adjustment for BMI, ${ }^{365,375,386,387}$ and that daytime and nocturnal BP was higher in patients with OSA compared to habitual snorers. ${ }^{376,388} \mathrm{~A}$ morning BP surge, defined as the difference in BP from the nadir during sleep to the two hours after waking, has been associated with risk of coronary events and ischemic stroke ${ }^{389,390}$ and even death in already hypertensive patients. ${ }^{391}$ Morning BP surges have been found to be significantly larger in untreated essential hypertension patients with OSA compared to those without OSA. ${ }^{373}$ Within pregnancy, very few studies have attempted to associate BP with SDB. Yinon et al. ${ }^{184}$ reported a significant correlation between RDI and mean BP in preeclamptic women, whereas Pien et al. ${ }^{7}$ found no such association in a mostly normotensive sample. Reid et al. ${ }^{274}$ failed to demonstrate a significant improvement in BP amongst GH women following treatment of mild SDB with CPAP.

Our results showed that within the GH group, systolic BP rose slightly from evening until morning for those with SDB, whereas those without SDB had the anticipated drop in systolic BP. However, the magnitude of $\mathrm{BP}$ change overnight was small. Within the PE and control groups, late evening and early morning BP was unaffected by SDB and neither systolic nor diastolic BP changed overnight irrespective of SDB status. Given the physiological and clinical evidence to support surges in nocturnal BP and raised daytime $\mathrm{BP}$ resulting from obstructive events and hypoxia in SDB, why did our results within the $\mathrm{PE}$ and control groups not support our hypotheses? Firstly, it may be that the morning BP measurement was delayed too long after awakening, and so the acute effects of SDB on BP had been attenuated. In our protocol, we endeavoured to take the BP measurements as soon as possible upon awakening; however, this was not always the case. At times, the pregnant woman had already been awake for a time before PSG recording stopped, or a bathroom break was urgently required. Prior studies have shown that if sleep is interrupted sufficiently, BP returns to waking levels ${ }^{392}$ and BP changes upon awakening occur rapidly. ${ }^{393}$ In illustration of this, Edwards et al. ${ }^{270}$ noted that one of their PE patients woke for 3 hours in the middle of their PSG, with their BP returning to normal during this time before rising again once asleep.

Secondly, BP recordings were taken at a single time point on one night and the following morning only. It should also be considered that BP measurements performed by research staff in a clinical setting may differ from those taken by the patient in her home. As mentioned, the level of SDB within our sample was relatively mild compared to those analysed in non-pregnant populations, and the phenotype of PE within our sample was less severe than those featured in the Edwards et al. studies above, ${ }^{269,270}$ 
where all participants were inpatients admitted with severe PE, and the women had marked rises in their $\mathrm{BP}$ in response to obstructive respiratory events and across the night.

\subsubsection{Severity of Sleep-Disordered Breathing}

A potential explanation for findings that were contrary to our original hypothesis was the severity of SDB within our sample. Our primary outcomes were analysed using a low-threshold definition of SDB as RDI $\geq 5$, and of those that did have SDB in our study, almost two-thirds were only mild. Many studies relating SDB to hypertension and cardiovascular disease have shown a dose-response relationship. ${ }^{65,365,386}$ In a study of perimenopausal women, it was only those with moderate to severe SDB (AHI $\geq 15)$ rather than mild SDB, who had a higher prevalence of hypertension and higher 24-h ambulatory BP than those without SDB. ${ }^{127}$ A recent review study concluded that severe SDB ( $\mathrm{HHI} \geq 30 / \mathrm{hr}$ ) is strongly associated with increased cardiovascular disease, however risk from moderate SDB (AHI 15-30/hr) is uncertain and there is no conclusive evidence of increased cardiovascular risk from mild SDB $(\mathrm{AHI}<15 / \mathrm{hr}) .{ }^{74}$ Given the preponderance of mild disease amongst our participants, this may have diluted any effect of SDB across our whole cohort.

Another reason for the absence of association between SDB and HDP may relate to focusing on frequency of respiratory events rather than measures of hypoxaemia. The theory of shared pathophysiology between SDB and PE depends highly on the consequences of intermittent hypoxia following apnoea. For example, Peled et al. ${ }^{394}$ found that the degree of nocturnal hypoxia rather than RDI was more closely associated with sympathetic activation and daytime BP and a large European cohort study demonstrated that ODI was independently associated with prevalent hypertension whereas AHI was not. ${ }^{395}$ Nonetheless, although the median RDI of our sample was 4.5 , the ODI $3 \%$ and ODI $4 \%$ were only 1.8 and $0.5 / \mathrm{hr}$ respectively and this low degree of hypoxia may go towards explaining why SDB in pregnancy did not have an impact on severity of hypertensive disease or BP.

Even so, there is evidence to suggest that respiratory events without hypoxia have cardiovascular consequences. 396,397 In preeclamptic women with mild SDB characterised by upper airway flow limitation only, reductions in nocturnal BP and minimisation of cardiac output reductions associated with PE have been seen with CPAP treatment, ${ }^{169,270}$ suggesting that even mild SDB is associated with adverse hemodynamic changes in women with PE. The lack of hypoxaemia in our sample may only be part of the reason for our negative results. Perhaps a more likely explanation is a combination of the milder degree of respiratory events and hypoxia experienced by this sample and the potentially transient nature of SDB in pregnancy discussed earlier.

In a planned sub-analysis, we examined our results at an increased SDB severity level of RDI $\geq 15$ and there were still no relationships to suggest that SDB has a negative impact on any measure of hypertensive disease in pregnancy. In fact, it is possible that women with moderate to severe SDB unexpectedly have a better outlook, in that the three preeclamptic women with an RDI $\geq 15$ had a significantly later gestation at delivery compared to those without SDB, and those with GH/PE and an 
$\mathrm{RDI} \geq 15$ had lower levels of PE severity markers ET-1 and sEng at delivery. Although not significant, the PE and SDB group also had twice as many days from initial diagnosis of PE and delivery.

These findings are important for us to consider. Keeping in mind that these could simply be a result of small numbers or a reflection of multiple comparisons, it remains intriguing that these outputs all point in the same direction. It is plausible that hypertensive disease where SDB plays a role in later placental dysfunction might have a better course than PE where poor initial placentation with ongoing uteroplacental ischaemia/hypoxia is a key feature, because at least the placenta and maternal endothelium gets re-oxygenation during the day and intermittently at night. In other words, we could speculate that there are 'different sorts' of PE - one in which the pathogenesis is earlier in the pregnancy and is associated with primary placental ischaemia, and another more benign subtype wherein the initial placentation process is essentially normal but SDB-linked hypertension exacerbates the second phase of PE involving sympathetic activation, inflammation, oxidative stress and widespread endothelial dysfunction. Future research into this novel concept may be worthwhile as further prolongation of gestation for these women with SDB-linked hypertensive disease may be plausible.

We had $18 \%$ of our overall sample with an $\mathrm{RDI} \geq 15$, which is a high proportion compared to previous studies in pregnancy. ${ }^{7,123}$ Our results may have been somewhat diluted due to three women with severe SDB (RDI's of 149, 108 and 83/hr) commencing CPAP during their pregnancy and being removed from some analyses. However, the two most severe SDB participants within the GH group (RDI 69 and 55/hr) did not go on to develop PE, and the three normotensive women who developed GH had RDI's below 10.

\subsubsection{Considerations of the Study}

Allocation into SDB groups for this study was based on a sleep study performed in the middle of the third trimester, at an average of 33 weeks gestation. We acknowledge however that SDB status may have been different for some women depending on when the sleep study was done. We know from previous studies that severity of SDB does change across pregnancy, however the magnitude of this change is generally minor. For example, in a sample stratified by BMI, Pien et al. ${ }^{7}$ demonstrated that mean AHI significantly increased from 2.07 events/hr in the first trimester to 3.74 events $/ \mathrm{hr}$ in the third trimester. Despite this small relative increase, this translated into an increase in the proportion of those with $\mathrm{AHI} \geq 5$ from $10.5 \%$ to $26.7 \%$. The results were similar within a high-risk pregnant cohort, with RDI increasing from 8.9 to 11.9 from early pregnancy to the third trimester, increasing the proportion of those with $\mathrm{RDI} \geq 5$ from $30 \%$ to $47 \%$. Within this study, new onset of SDB in the third trimester occurred in $20 \%$ of the sample, with the majority of cases being mild. ${ }^{122}$

Despite this, it is highly likely that those with moderate and severe SDB towards the end of pregnancy would have had it prior to pregnancy or at least from the early stages, and hence our persistent negative results when defining SDB at higher levels of severity should be more representative of more chronic effects of SDB on hypertensive disease in pregnancy. 


\subsubsection{Strengths and Limitations}

Our study is amongst the first to look longitudinally at how SDB impacts on measures of the diagnosis and severity of hypertensive disease in pregnancy. Most studies to date have simply compared the presence of the conditions of GH and PE in those with versus without SDB,7,8,123,124,260 but have not looked at key outcomes such as longitudinal BP control and antihypertensive use, gestation at diagnosis and prolongation of pregnancy following diagnosis. We are also the first study to look at angiogenic markers in a pregnant cohort with SDB.

A key strength of our study over others was the use of full gold standard PSG to measure SDB, rather than abbreviated monitoring techniques. ${ }^{122-124,184,259}$ This allowed us to most accurately designate pregnant women by SDB status, and to include subtle respiratory events to be included when calculating severity of disordered breathing. The initial BMI-matched design of our study also allowed us to somewhat control for the influence of obesity - the BMI measured at the time of the sleep study was not considerably different across those that had and did not have SDB.

The most notable limitation of our study is sample size. The sample size calculation was based on our primary outcome in Chapter 3, which compared the frequency of SBD in HDP to normotensive pregnancy. The analyses undertaken in this chapter were limited by smaller sample size, given we decided to split the GH and PE groups given their very different pathophysiology. This means they need to be interpreted with some caution. The very wide IQR's for the angiogenic results, for example, suggests high variability amongst pregnant women. A larger sample size would have strengthened these findings by allowing us to look at individual sub-groups with more rigour.

Another limitation of our study relates to the logistics and timing of obtaining the maternal blood samples. The third trimester blood sample was relatively easy to collect from the participant at an antenatal appointment, but the maternal blood samples at delivery were often missed for a number of reasons generally out of our control (see Section 4.2.4 - Statistical Analysis page 90). As a consequence, we collected 21 blood samples in the third trimester that did not have a matching delivery sample and were therefore not analysed.

Finally, from a statistical analysis perspective, we were only able to measure SDB status at one timepoint during pregnancy which is not ideal for survival analysis and mixed modelling. It is unlikely the $\mathrm{RDI}$ stays completely static across pregnancy, however it was not feasible to perform PSG multiple times across pregnancy in a cohort study such as ours. Allocating SDB status as measured in the third trimester via gold standard PSG was our best option and it is unlikely that this significantly affected our results. 


\subsubsection{Conclusion}

Our study is amongst the first to look specifically at how SDB impacts on clinical, biochemical and antiangiogenic measures of hypertensive disease in pregnancy. We found that the presence of SDB overall did not influence the course of hypertensive disease in pregnancy. Specifically, mild or at least moderate SDB did not impact gestation at diagnosis of GH or PE, or measures of the maternal syndrome of increased BP, renal insufficiency and proteinuria, low platelet count and liver dysfunction. Angiogenic markers, which are thought to best characterise severity of PE, were not adversely affected by SDB. Conversely, we were intrigued to find a number of favourable outcomes for HDP women with moderate to severe SDB, leading us to speculate whether a more benign subtype of PE linked to SDB may exist.

Given the number of studies confirming the relationship between SDB and diagnosis of HDP, the causal pathways still require further study. Better understanding of this relationship will be informed by future research focussing on more severe levels of SDB, and with SDB pre-dating pregnancy. Nevertheless, cause and effect will always be difficult to completely disentangle, particularly given the potential contribution of residual confounders, particularly with obesity. Ultimately, the impact of SDB on pregnant women and their infants may only be determined through an interventional treatment trial, and measuring its success in preventing or prolonging pregnancies affected by hypertensive disorders. 


\subsection{Supplementary Tables}

Table 4.8 Third Trimester (A), Delivery (B) and Change Per Week Values for Endothelin-1, Soluble Flt-1, Soluble Endoglin and Placental Growth Factor for the Preeclampsia versus Gestational Hypertension Groups, and the Gestational Hypertension/Preeclampsia versus Control Groups

\begin{tabular}{|c|c|c|c|c|c|c|}
\hline & PE v & $\mathrm{GH}$ & & $\mathrm{GH} / \mathrm{PE}$ & Controls & \\
\hline & $\begin{array}{c}\text { PE } \\
(n=5)\end{array}$ & $\begin{array}{c}\mathrm{GH} \\
(\mathrm{n}=18)\end{array}$ & $\mathrm{p}$ & $\begin{array}{l}\mathrm{GH} / \mathrm{PE} \\
(\mathrm{n}=23)\end{array}$ & $\begin{array}{l}\text { Controls } \\
(n=24)\end{array}$ & $\mathrm{p}$ \\
\hline ET-1 A & $1.9(1.2,2.1)$ & $1.4(1.0,1.8)$ & .36 & $1.5(1.0,2.0)$ & $1.9(1.3,2.6)$ & .083 \\
\hline ET-1 B & $1.5(1.2,2.3)$ & $1.9(1.0,2.3)$ & .64 & $1.9(1.1,2.3)$ & $2.5(1.7,3.0)$ & .03 \\
\hline ET-1 change $p / w$ & $-0.17(-0.49,0.41)$ & $0.15(-0.10,0.41)$ & .20 & $0.11(-0.17,0.36)$ & $0.06(-0.03,0.14)$ & .87 \\
\hline sFlt-1 A & $\begin{array}{c}8990.5 \\
(7048.6,18110.8)\end{array}$ & $\begin{array}{c}4188.1 \\
(2604.4,7103.2)\end{array}$ & .02 & $\begin{array}{c}5492.7 \\
(3158.1,8990.5)\end{array}$ & $\begin{array}{c}957.9 \\
(487.8,2161.7)\end{array}$ & $<.001$ \\
\hline sFlt-1 B & $\begin{array}{c}8805.5 \\
(6944.2,26080.1)\end{array}$ & $\begin{array}{c}6231.6 \\
(3655.0,9550.6)\end{array}$ & .11 & $\begin{array}{c}6837.3 \\
(4163.5,10023.6)\end{array}$ & $\begin{array}{c}3416.5 \\
(2299.0,5107.1)\end{array}$ & $<.001$ \\
\hline sFlt-1 change $p / w$ & $105.8(-424.9,7106.5)$ & $679.2(85.9,1191.8)$ & .69 & $611.4(80.3,1154.8)$ & $357.1(184.1,640.9)$ & .26 \\
\hline sEng A & $35.2(23.0,49.7)$ & $15.8(6.7,22.5)$ & .01 & $19.6(7.9,33.5)$ & $7.1(3.7,9.3)$ & $<.001$ \\
\hline sEng B & $38.3(21.1,58.4)$ & $14.6(8.2,31.2)$ & .03 & $17.9(9.7,34.9)$ & $9.8(6.3,21.2)$ & .01 \\
\hline sEng change $\mathrm{p} / \mathrm{w}$ & $-0.5(-3.2,16.5)$ & $0.4(-0.6,2.0)$ & .86 & $0.2(-0.8,2.9)$ & $0.7(0.2,1.7)$ & .46 \\
\hline PIGF A & $50.6(27.2,88.1)$ & $103.6(64.2,130.7)$ & .06 & $85.2(52.4,116.1)$ & $182.6(120.3,264.2)$ & $<.001$ \\
\hline PIGF B & $51.0(26.1,91.0)$ & $84.0(53.4,106.5)$ & .20 & $81.8(46.3,97.7)$ & $92.6(58.1,156.1)$ & .18 \\
\hline PIGF change $p / w$ & $-1.2(-3.8,2.3)$ & $-8.4(-22.7,0.3)$ & .15 & $-4.9(-19.9,0.4)$ & $-13.7(-34.6,-6.1)$ & .03 \\
\hline
\end{tabular}

Note. Values given as Mdn (IQR). PE = preeclampsia, GH = gestational hypertension, ET-1 = endothelin-1, sFlt-1 = soluble fms-like tyrosine kinase 1, sEng = soluble endoglin, PIGF = placental growth factor, $\mathrm{p} / \mathrm{w}=$ per week. 
Endothelin-1

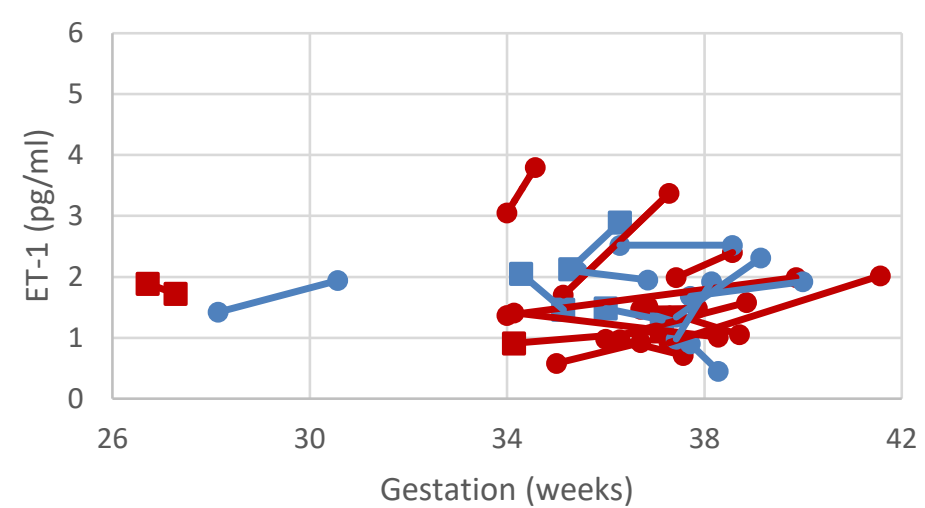

Soluble Endoglin

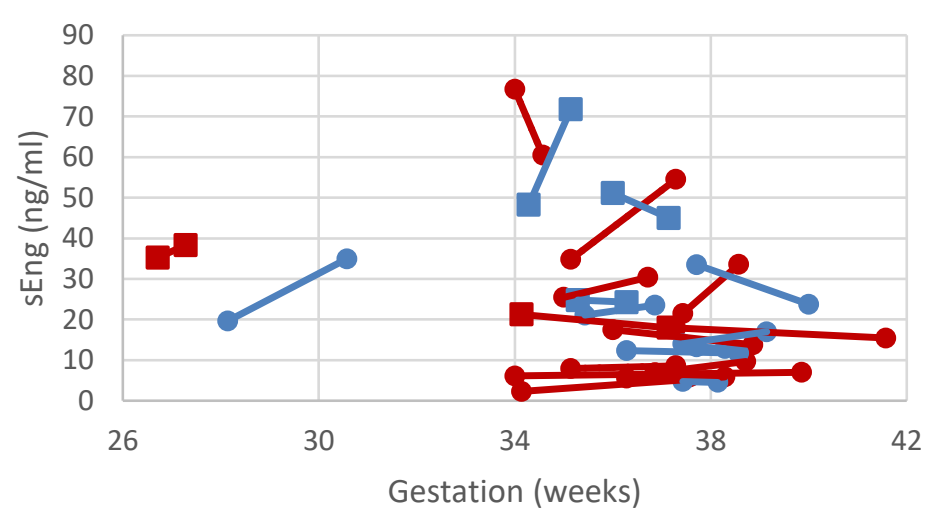

Soluble Flt_1
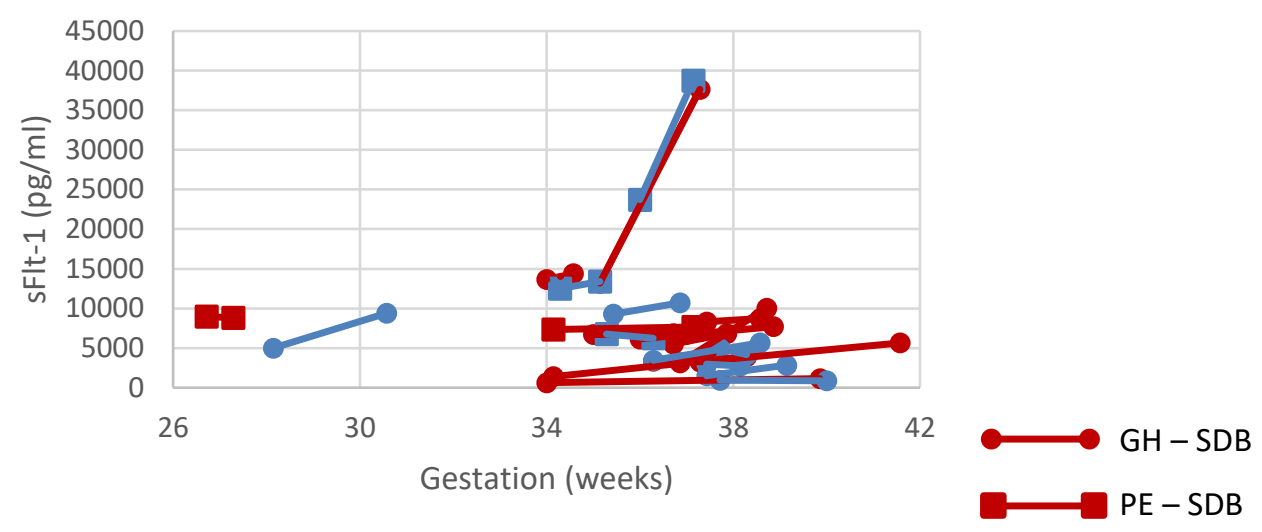

Placental Growth Factor

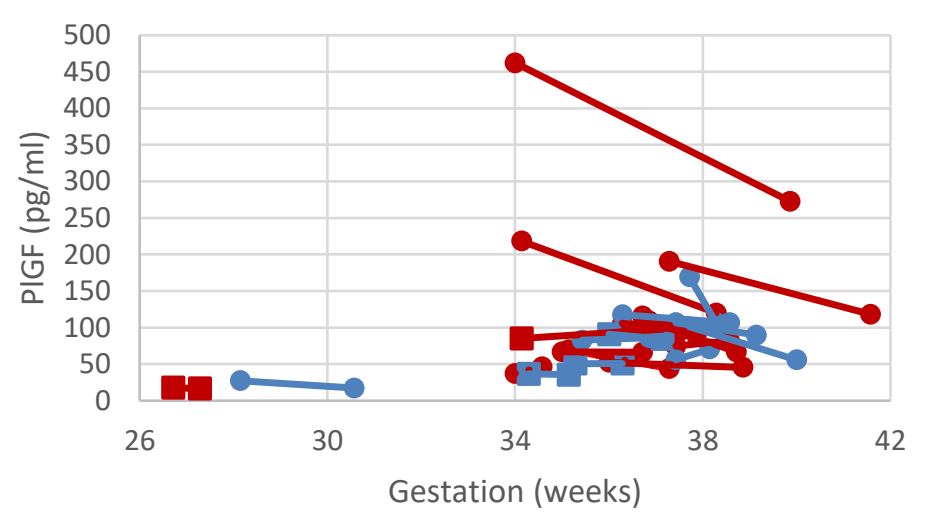

$\longrightarrow \mathrm{GH}-\mathrm{no}$ SDB

$\longrightarrow P E-n o S D B$

Figure 4.12 Endothelin-1 (ET-1), soluble fms-like tyrosine kinase-1 (sFlt-1), soluble endoglin (sEng) and placental growth factor (PIGF) measured in the third trimester and at delivery for each participant in the GH/PE group - stratified by SDB (RDI $\geq 5)$. The horizontal axis indicates the gestation at which the third trimester and delivery samples were collected. $G H=$ gestational hypertension, $P E=$ preeclampsia, $S D B=$ sleep-disordered breathing. 
Endothelin-1

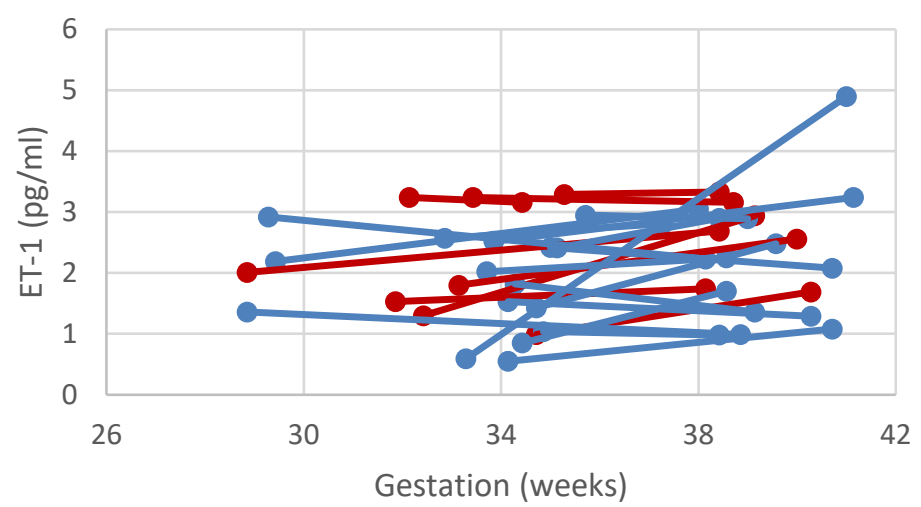

Soluble Endoglin

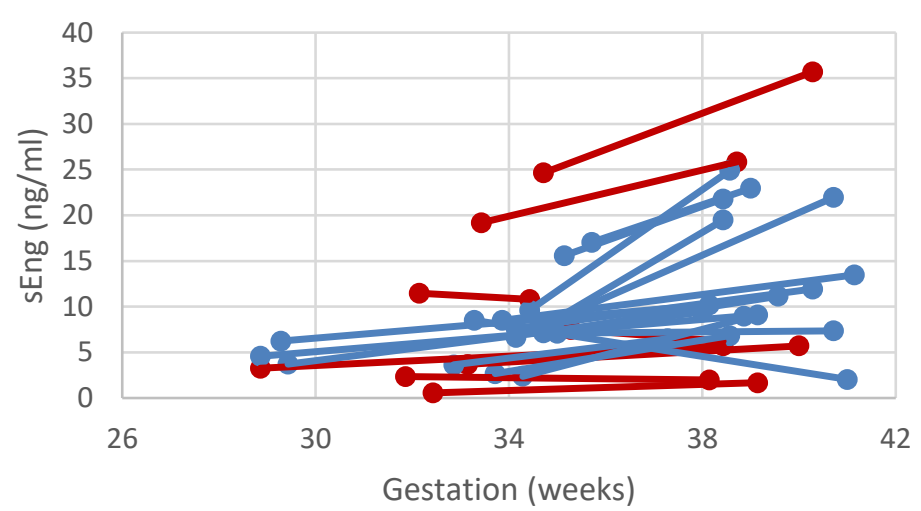

Soluble Flt_1

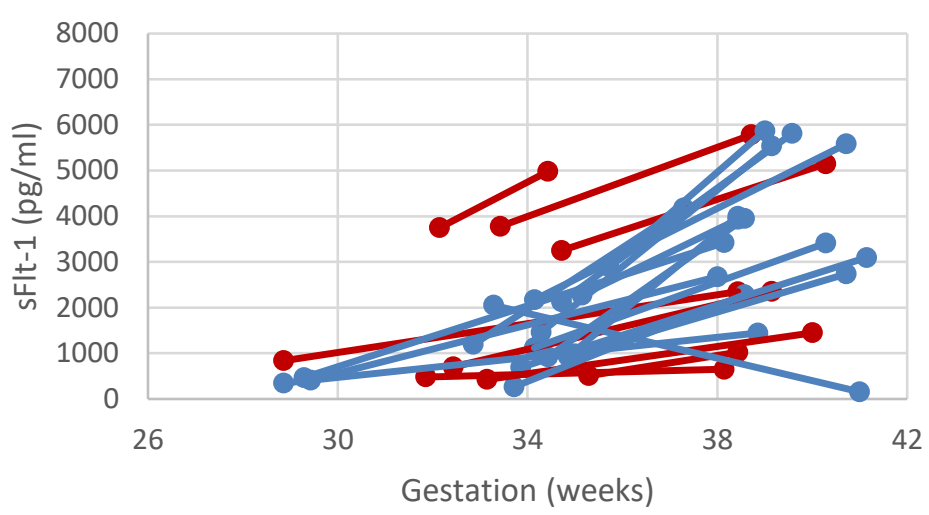

Placental Growth Factor

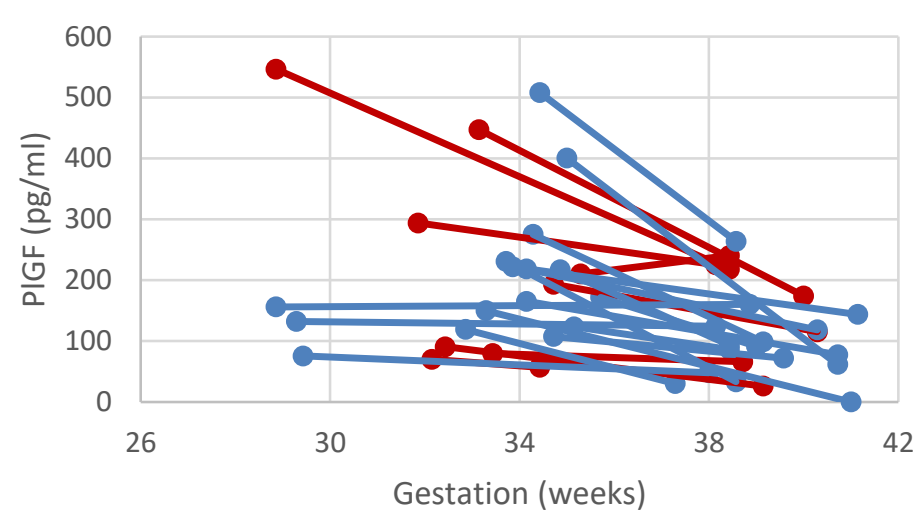

Figure 4.13 Endothelin-1 (ET-1), soluble fms-like tyrosine kinase-1 (sFlt-1), soluble endoglin (sEng) and placental growth factor (PIGF) measured in the third trimester and at delivery for each participant in the control group - stratified by SDB (RDI $\geq 5)$. The horizontal axis indicates the gestation at which the third trimester and delivery samples were collected. SDB = sleep-disordered breathing. 
Chapter 5 -

The Impact of Sleep-Disordered Breathing in Pregnancy

On Immediate and Long-Term Fetal Outcomes 


\section{Preface - Chapter 5}

Given that hypertensive disease during pregnancy is associated with fetal compromise, it is important to identify whether these already at-risk fetuses will be further disadvantaged by concurrent maternal SDB. The aim of Chapter 5 was to clarify whether the presence of SDB influenced the acute and chronic health of the fetus in women with HDP, as well as in normotensive women. As with Chapter 4, this chapter includes participants who were excluded from Chapter 3 for clinical suspicion of SDB, as Chapter 5 focuses on the sequalae of SDB on fetal health. This chapter is currently unpublished but has been written in a style to facilitate submission to an appropriate peer-reviewed journal in due course. 


\subsection{Introduction}

In Chapter 4, SDB appeared to have minimal impact on the maternal course and severity of hypertensive disease in pregnancy. However, HDP, particularly PE, are associated with impaired fetal growth and compromise, ${ }^{144-147}$ therefore the potential exists for SDB to further exacerbate the fetus already at risk of compromise. As discussed in Chapter 1, SDB is hypothesised to impact on fetal health in two ways (see Figure 5.1). Firstly, respiratory events with repeated episodes of hypoxaemia and cortical arousal lead to sympathetic activation and endothelial dysfunction. This could contribute to placental dysfunction and subsequently impaired fetal growth; compromised uteroplacental perfusion with or without repeated episodes of maternal hypoxaemia may result in insufficient supply of nutrients and oxygen to the fetus. ${ }^{242}$ Impaired fetal growth is associated with short and long-term complications including increased risks of intrapartum asphyxia and pre-term birth, respiratory distress syndrome, low Apgar scores, hypoglycaemia and seizures, and impaired neurological and cognitive development into childhood. ${ }^{398-401}$ Most significantly, more than one half of unexplained stillbirths have been confirmed to be associated with FGR. ${ }^{402,403}$

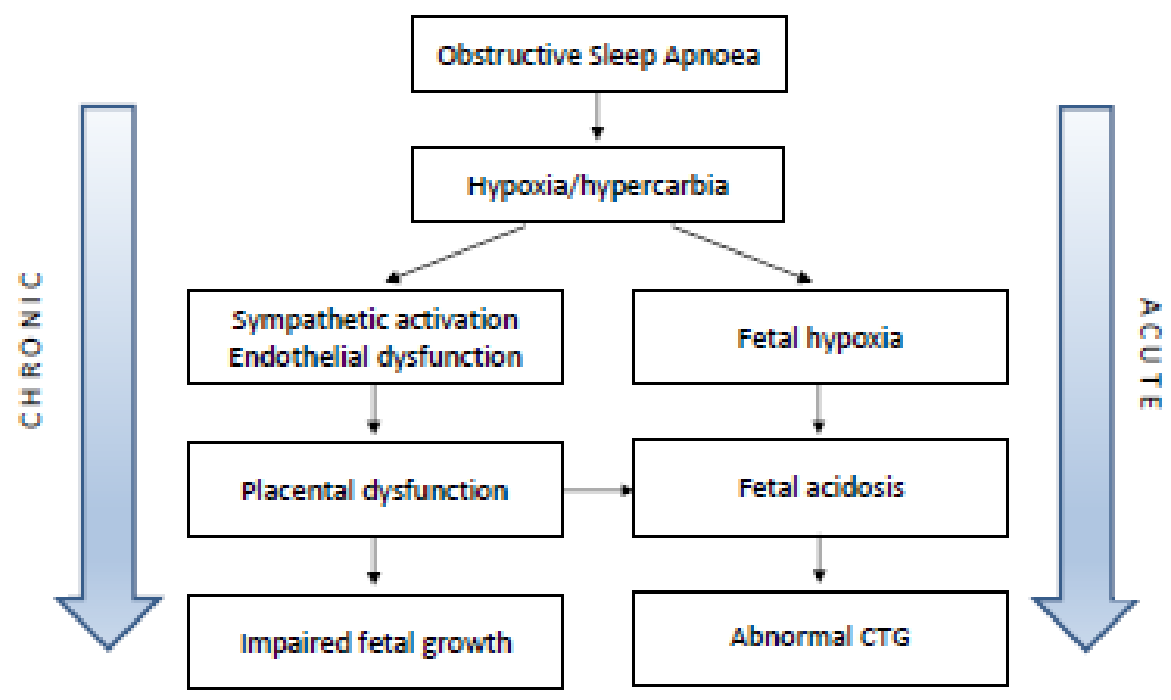

Figure 5.1 Potential interaction between SDB and acute and chronic fetal compromise. CTG = cardiotocogram. ${ }^{49}$

The second way in which maternal SDB may impact fetal health is by maternal hypoxaemia leading to fetal hypoxia, and if sufficiently severe, resulting in fetal acidosis (Figure 5.1). Even small declines in maternal oxygenation may endanger oxygen delivery to the fetus, particularly in the setting of underlying uteroplacental insufficiency. Further, any cause of maternal hypercapnia quickly leads to fetal respiratory acidosis. ${ }^{236}$ Fetal acidosis may result in asphyxial damage with potential long-term 
neurodevelopmental impairment and stillbirth. ${ }^{235,237,404}$ Fetal heart rate analysis (CTG) is the current means by which a fetus is evaluated for adequacy of oxygenation in real time. ${ }^{244}$

The few studies that have investigated the impact of objectively-measured SDB on the health and growth of the fetus have reported mixed results. SDB during pregnancy has been associated with detrimental outcomes such as impaired fetal growth and preterm birth,242,262,280 neonatal ICU admission, ${ }^{124}$ reduced nocturnal fetal movements ${ }^{284}$ and fetal distress on nocturnal CTG. ${ }^{240}$ On the other hand, no differences in fetal growth, birthweight, preterm delivery $7,262,263,280$ and measures of nocturnal fetal distress ${ }^{107,242}$ have also been reported between pregnant women with and without demonstrated SDB. No studies to date have investigated the potential effect of SDB on the health of the fetus among mothers diagnosed with HDP.

\subsubsection{Aims and Hypotheses}

In this study, we aimed to see whether the presence of SDB influenced the health of the fetus in women with HDP. Specifically, we hypothesised that

i) women with GH or PE and SDB have short- and long-term evidence of more fetal compromise compared to women with PE/GH without SDB, evidenced by -

a.abnormal CTG during respiratory events in sleep

b.impaired fetal growth in late pregnancy and/or FGR

c.alterations in fetal growth hormones (the IGF axis)

d.worse perinatal outcomes re: Apgar scores, NICU/SCN admission etc.

ii) normotensive control women with SDB have short- and long-term evidence of more fetal compromise compared to control women without SDB, evidenced by -

a.abnormal CTG during respiratory events in sleep

b.impaired fetal growth in late pregnancy and/or FGR

c.alterations in fetal growth hormones (the IGF axis)

d.worse perinatal outcomes re: Apgar scores, NICU/SCN admission etc.

\subsection{Method}

\subsubsection{Study Participants}

As described in Chapter 2 - Method Section 2.1 page 59, this study was initially a prospective casecontrol study. Cases included women with a singleton pregnancy diagnosed with GH or PE not requiring immediate delivery between 26 and 37 weeks gestation, and control participants were normotensive women one-to-one matched by BMl and gestational age to each of the cases (within $\pm 4 \mathrm{~kg} / \mathrm{m}^{2}$, measured at the first antenatal appointment and within \pm 4 weeks of gestational age), with uncomplicated pregnancies. To evaluate the impact of SDB on fetal outcomes related to GH and PE, participants were 
further divided into those with and those without SDB. Hypertension in pregnancy, and diagnosis of GH and PE were defined as per Chapter 2 - Method Section 2.1 page 59.

\subsubsection{Investigational Measures}

\subsubsection{Polysomnography}

Full overnight PSG was conducted either in the Austin Health sleep laboratory using the Compumedics E series (Abbotsford, Victoria, Australia), or in the participant's home with the Somté (Compumedics) portable sleep-monitoring device. Participants who were inpatients at the Mercy Hospital for Women were also studied using the portable Somté device. See Chapter 2 - Methods Section 2.2.1.1 page 60 for more detailed information on signals used and sleep and respiratory scoring criteria.

\subsubsection{Fetal Heart Rate Monitoring}

Continuous CTG was performed using the Monica AN24 fetal heart rate monitor (Monica Healthcare Ltd). The Monica AN24 is a non-invasive monitor requiring the placement of 5 adhesive electrodes onto the maternal abdomen, to monitor fetal ECG, maternal ECG and uterine EMG to measure uterine contractions (See Chapter 2 - Method Section 2.2.2.1 page 63 for further details and diagrams). The Monica AN24 is a single set up device designed to require no adjustment or repositioning of the electrodes, making it suitable for overnight continuous CTG recording. The leads are connected to a small portable unit where the data is stored until the recording is completed and is downloaded to the Monica VS software for review. In order to time synchronise the Monica data with the PSG, a marker was inserted simultaneously on both the CTG and PSG recordings.

\subsubsection{Ultrasound}

Ultrasound was performed in the third trimester of pregnancy to monitor fetal growth. These ultrasounds were performed by obstetricians with sub-specialist training in diagnostic ultrasound. The formula used for calculating EFW was based on measurements of head size (head circumference and biparietal diameter), abdominal circumference and femur length. ${ }^{223}$ The ultrasound EFW was customised for maternal height, pre-pregnancy (or if unknown, early pregnancy) weight, ethnicity, parity and fetal sex using the Australian dataset of the GROW software (www.gestation.net). ${ }^{299}$ Further details are given in Chapter 2 - Method Section 2.2.2.2 page 64.

\subsubsection{Blood Sampling}

Venous cord blood was collected at delivery and analysed for the fetal growth regulators IGF-1, IGF-2, IGFBP-1 and IGFBP-2. Free IGF-1 and IGF-2 determinations were performed using MILLIPLEX MAP Human IGF-I, II Magnetic Bead Panel kit (Millipore, Billerica, MA, USA). To separate IGFs from their binding proteins, all plasma samples underwent an acid-ethanol extraction procedure before measurement according to the manufacturer's instructions. All assays were read using the Bio-Plex workstation (Bio-Rad Laboratories, Hercules, CA) and results analysed with Bio-Plex Manager (version 
4.1.1) software. Intraassay and interassay coefficients of variation were less than $10 \%$. In addition, the values of the quality controls provided in the kits read within the specified ranges.

\subsubsection{Medical Records for Fetal Outcomes}

Following delivery, birthweight was customised as previously described using the GROW software (www.gestation.net), ${ }^{299}$ so that EFW and birthweight centile were adjusted for gestational age and relevant maternal characteristics.

Medical records were reviewed for the following delivery and fetal outcomes: gestation at delivery, customised birthweight centile, mode of delivery, Apgar scores at $1 \mathrm{~min}$ and $5 \mathrm{~min}$, and infant admission to NICU or special care nursery (SCN).

\subsubsection{Procedures}

As described in Chapters 3 and 4, participants with GH and PE underwent overnight PSG. Each control was matched within \pm 4 weeks of gestational age of a case. Approximately one hour prior to PSG setup, the Monica AN24 fetal heart rate monitor was applied to the maternal abdomen. Signal quality was confirmed for at least $15 \mathrm{~min}$ in the supine and non-supine position, with electrodes repositioned as required. CTG was time synchronised to the overnight sleep study to enable correlation of any fetal heart rate abnormality with objectively confirmed respiratory events and/or oxygen desaturations. All fetal heart rate traces were reviewed the following morning by a single observer (Alison Fung) blinded to maternal hypertensive status and PSG results, to ensure no abnormalities were present that needed further follow-up and management. At the time of the sleep study, height and weight were also recorded.

All women underwent ultrasound for fetal growth between 32-34 weeks gestation (or as soon as practicable if recruited after 34 weeks gestation). Ultrasound was performed at this time to enable sufficient time between third trimester growth assessment and delivery for any significant change in customised centile suggestive of slowing of the fetal growth trajectory.

At delivery, venous blood from the umbilical cord was collected. Participants were given a blood tube pack and pathology slips to take with them when admitted to have their baby. If admission for delivery was during business hours a research midwife collected the cord blood sample after delivery of the baby, otherwise delivery room/operating theatre staff were required to collect blood. The relevant birth and fetal outcome measures were obtained from medical histories following delivery.

\subsubsection{Statistical Analysis}

All statistical analyses were performed with SPSS 21.0 (SPSS Inc., Chicago, Illinois). Data were assessed for linearity and normality by obtaining skewness and kurtosis values and by checking distributions on histograms and normal probability plots. Values are given in means with standard 
deviations $(M \pm S D)$ or median and interquartile range $(M d n(I Q R))$ for non-normally distributed variables. A two-sided $p$ value of less than 0.05 was considered to indicate statistical significance.

The GH and PE groups were combined for data analyses in this chapter and referred to as the HDP group, as there were no differences in the effect of SDB on hypertensive disease severity found between the GH and PE groups in Chapter 4. SDB was defined as an RDI of $\geq 5$ events per hour, and the HDP and control participants were split into the SDB group and the No SDB group. Secondary analyses were performed with SDB defined as RDI $\geq 15$.

Comparisons between the SDB and No SDB groups on outcomes of fetal wellbeing were done using Fisher's exact test of independence for categorical variables, independent-samples t-tests for normallydistributed continuous variables and Mann-Whitney $U$ tests for non-normally distributed continuous variables. Supplementary comparisons between the PE, GH and control groups were performed using univariate analysis of variance (ANOVA).

The CTGs were time synchronised to the PSG and manually analysed for rate of successful fetal heart rate tracing during sleep in 15 second epochs, so that percentage of CTG success was calculated as 'minutes of successful fetal heart rate trace during sleep / minutes of sleep * 100' to give a value between 0 and $100 \%$. The criteria for CTGs to be included in further analysis was i) an overall CTG success rate of $>50 \%$, and ii) at least $3 \times 1$-hour blocks with $>50 \%$ asleep and $>80 \%$ successful fetal heart rate trace. This inclusion criterion was chosen as the fetal heart rate was difficult to interpret with large sections of missing data and there was potential for fetal heart rate events to be missed if $>20 \%$ of the fetal heart rate was absent.

Each CTG was analysed by an obstetrician to identify pre-determined fetal heart rate events potentially signifying fetal distress during maternal sleep, as follows -

1. Event 1 - prolonged bradycardia (defined as $>15$ beats per minute below baseline for more than or equal to 90 seconds and less than 5 minutes).

2. Event 2 - recurrent severe variable decelerations (defined as a fall of $>60$ beats per minute from previous baseline and of $>60$ seconds duration, and at least 2 per 2 hours).

3. Event 3 - repeated unprovoked decelerations or late decelerations (i.e. decelerations following uterine contraction) accompanied by tachycardia or loss of variability.

4. Event 4 - decelerations lasting between 60 and 90 seconds and dropping below the baseline by > 15 beats per minute.

Comparisons between the SDB and No SDB groups (overall and within the HDP and control groups) were made on the proportion of participants displaying each type of fetal heart rate event, and the 
average number of fetal heart rate events overnight amongst those who displayed fetal heart rate events on CTG.

Ordinal logistic regression was used to initially assess the univariate relationships between the rate of total fetal heart rate events per hour of sleep as an ordinal variable with three levels and SDB and demographic variables, and odds ratios (ORs) were calculated. In order to determine whether more severe levels of SDB affect nocturnal fetal distress, continuous RDI and ODI variables were subject to logarithmic transformation due to extreme skewness. The rate of fetal heart rate events per hour of sleep was divided into the following three categories -1) 0 events, 2) $>0$ to 0.5 events per hour, and 3) $>0.5$ events per hour. The assumption of proportional odds was met for each equation. Stepwise ordinal regression modelling was performed with fetal heart rate events as the ordinal dependent variable, and explanatory variables with an $\alpha$ of less than 0.20 on univariate analysis were included.

For assessment of fetal growth, fetal growth restriction (FGR) was defined as a customised birthweight $<10^{\text {th }}$ centile for gestational age. Evidence of slowed third trimester growth was defined as a fall in customised centile of greater than a third ( $33 \%$ decrease) between the third trimester ultrasound and birth. Impaired fetal growth was defined as either FGR or slowed third trimester growth.

Slowed third trimester growth was calculated only for those participants where at least two weeks had lapsed between the third trimester ultrasound and birth. A number of participants could therefore not be included in this analysis because pre-term birth supervened or they were recruited late to the study. The number of weeks/days between the third trimester ultrasound and birth was variable. To facilitate standardised comparison, a rate of change in customised centile per day was calculated for each participant (a positive value indicated an increase in growth trajectory and a negative value indicated a slowing in growth trajectory). This daily change in centile was then multiplied by 42 , to generate the estimated change in customised centile that would occur over exactly six weeks. This was chosen based on our previous study examining maternal SDB and its impact on fetal growth, ${ }^{242}$ and that the average time between the third trimester ultrasound ( 33 weeks +1 day) and birth $(38+5)$ in our sample was $5+4$ weeks. Significant slowing of fetal growth was defined as a fall of $>33$ centiles between the initial scan and birth 6 weeks later. While analysis of slowed third trimester growth could only be performed for those with a valid third trimester ultrasound, analysis of birthweight indices including fetal growth restriction was done for all participants.

Not all participants were able to have cord blood collected. The most common reasons for this were that delivery occurred overnight or on the weekend when research midwives were unavailable, precipitate labour (limited time to collect the sample) or consent not given by the participant

\subsection{Results}

A total of 87 pregnant women participated; a flowchart illustrating the data available at each step of analysis is shown in Figure 5.2. 


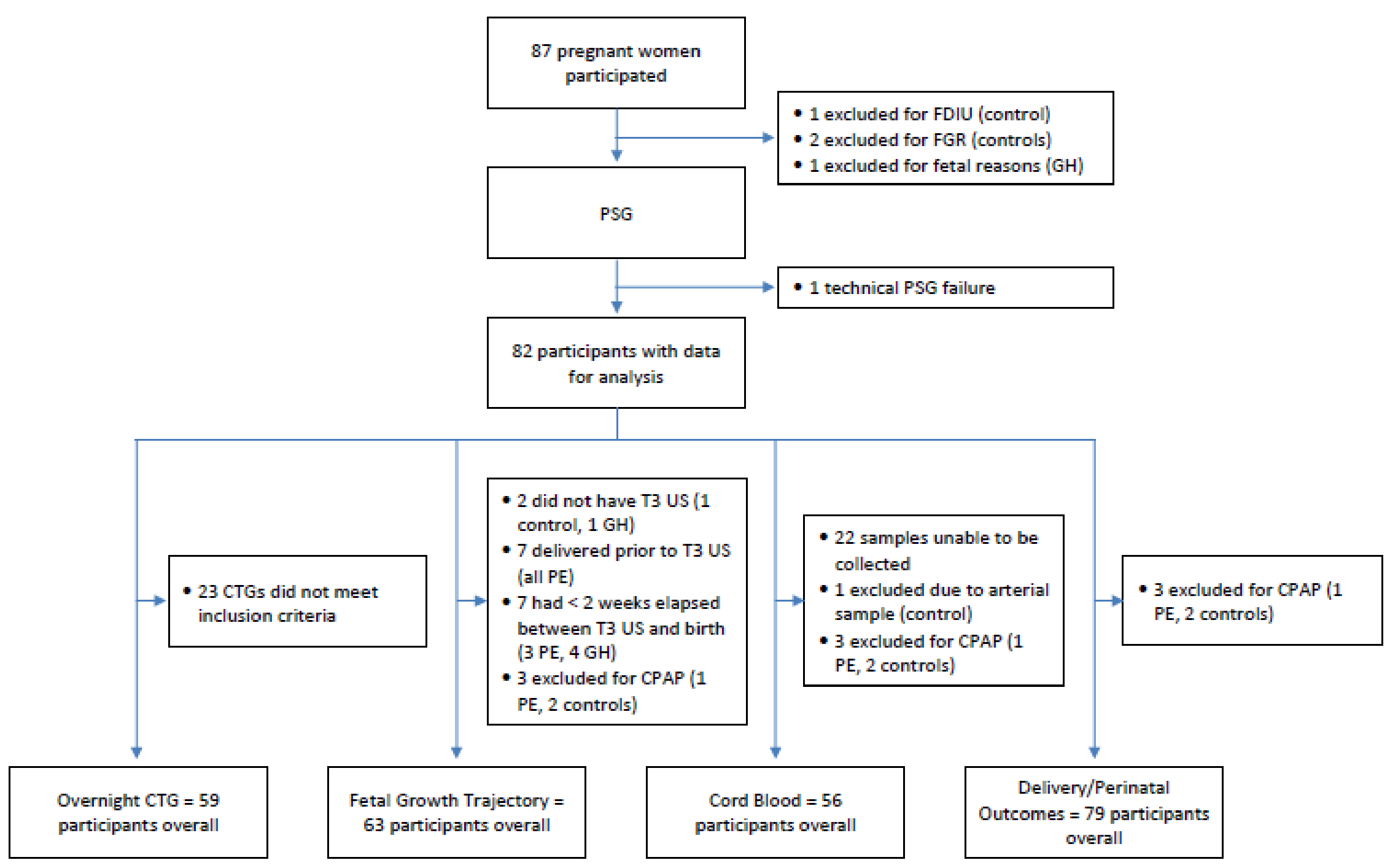

Figure 5.2 Flow chart of participant data available for each fetal outcome. FDIU = fetal death in utero, FGR $=$ fetal growth restriction, GH $=$ gestational hypertension, $P S G=$ polysomnography, $C T G=$ cardiotocography, $T 3=$ third trimester, $U S=$ ultrasound, $P E=$ preeclampsia, $C P A P=$ continuous positive airway pressure. 


\subsubsection{Demographics}

As per Table 5.1, 21 (52.5\%) of the HDP group and $16(38 \%)$ of the control group had SDB. Within each group, there were no differences in age, parity, GDM, and gestation at sleep study for the SDB versus No SDB groups. The BMI value measured at the first antenatal appointment was taken at a mean of $15.0 \pm 2.6$ weeks gestation; this and weight gain between the first appointment and PSG was not statistically different across groups, however BMI at the time of the sleep study was higher amongst those with SDB in the HDP group. As expected, those with SDB had a significantly higher RDI, ODI3\%, $\mathrm{ODI} \%$ and lower $\mathrm{SpO}_{2}$ nadir than those without SDB. Demographic details for the PE and GH groups separately are available in Supplementary Table 5.9

An in-laboratory sleep study was chosen by 24 participants and a home sleep study chosen by 40 participants, the remaining 18 participants were inpatients due to HDP (with no difference in RDI, in-lab $\operatorname{Mdn}(I Q R)=4.6(3.2-21.3)$ vs. home $=4.6(2.2-9.3)$ vs. inpatient $=3.8(1.8-12.4), p=.57)$. At the time of the sleep study, 23 of the hypertensive participants were taking anti-hypertensive medication; 16 were taking Labetalol, five were taking Aldomet, one was taking both Labetalol and Aldomet, and one was taking both Labetalol and Nifedipine.

After undergoing PSG in the third trimester of pregnancy, three participants who were diagnosed with severe SDB commenced CPAP - one participant with PE (RDI of 150.6) and two control participants (RDI of 108.8 and 83.6). These participants were subsequently excluded from all analyses apart from CTG analysis as this was measured prior to the commencement of CPAP (see Figure 5.2). 
Table 5.1 Demographics for Hypertensive Disorders of Pregnancy Cases and Normotensive Controls Stratified by SDB Status

\begin{tabular}{|c|c|c|c|c|c|c|}
\hline & \multicolumn{2}{|c|}{$\begin{array}{c}\text { HDP } \\
(n=40)\end{array}$} & \multicolumn{4}{|c|}{$\begin{array}{l}\text { Controls } \\
(n=42)\end{array}$} \\
\hline & $\begin{array}{c}\text { SDB } \\
(n=21)\end{array}$ & $\begin{array}{l}\text { No SDB } \\
(n=19)\end{array}$ & $p$ & $\begin{array}{c}\text { SDB } \\
(n=16)\end{array}$ & $\begin{array}{l}\text { No SDB } \\
(n=26)\end{array}$ & $p$ \\
\hline Age (years) & $33.3 \pm 4.7$ & $32.0 \pm 4.3$ & 38 & $33.5 \pm 3.9$ & $32.9 \pm 4.8$ & .67 \\
\hline Nulliparous & $14(66.7 \%)$ & $12(63.2 \%)$ & 1.0 & 9 (56.3\%) & $11(42.3 \%)$ & .53 \\
\hline GDM & 5 (23.8\%) & 3 (15.8\%) & .70 & $4(25.0 \%)$ & 5 (19.2\%) & .71 \\
\hline BMI first appt & $33.8 \pm 7.0$ & $30.7 \pm 7.4$ & .18 & $34.1 \pm 7.8$ & $31.8 \pm 6.4$ & .31 \\
\hline Weight gain $(\mathrm{kg})$ & $11.4 \pm 5.1$ & $8.6 \pm 6.4$ & .13 & $9.8 \pm 5.9$ & $9.5 \pm 5.5$ & .90 \\
\hline $\mathrm{BMI}$ at PSG & $38.1 \pm 6.4$ & $33.8 \pm 7.0$ & .047 & $37.9 \pm 6.8$ & $35.3 \pm 5.5$ & .18 \\
\hline $\begin{array}{l}\text { Gestation at } \\
\text { PSG (weeks) }\end{array}$ & $34.0 \pm 3.4$ & $33.1 \pm 3.6$ & .41 & $33.2 \pm 2.3$ & $32.9 \pm 2.6$ & .72 \\
\hline $\mathrm{RDI} / \mathrm{hr}$ & $\begin{array}{c}14.3(7.5 \\
37.7)\end{array}$ & $1.9(1.1,3.1)$ & $<.001$ & $9.2(7.5,28.8)$ & $2.9(1.3,4.0)$ & $<.001$ \\
\hline ODI $\geq 3 \%$ overall & $6.0(2.1,36.7)$ & $1.0(0.0,1.8)$ & $<.001$ & $8.7(1.1,29.1)$ & $1.0(0.2,2.5)$ & .004 \\
\hline ODI $\geq 4 \%$ overall & $3.3(0.4,23.5)$ & $0.1(0.0,0.8)$ & .001 & $3.2(0.3,12.7)$ & $0.3(0.0,0.5)$ & .002 \\
\hline $\mathrm{SpO}_{2}$ nadir $\%$ & $\begin{array}{c}88.0(81.0 \\
91.5)\end{array}$ & $\begin{array}{c}91.0(90.0 \\
93.0)\end{array}$ & .009 & $\begin{array}{c}89.5(86.0 \\
90.8)\end{array}$ & $\begin{array}{c}91.0(90.0 \\
93.0)\end{array}$ & .002 \\
\hline
\end{tabular}

Note. Values given as $\mathrm{M} \pm \mathrm{SD}$, Mdn (IQR), or $\mathrm{n}(\%)$. SDB = sleep-disordered breathing, HDP = hypertensive disorders of pregnancy, GDM = gestational diabetes mellitus, $\mathrm{BMI}=$ body mass index $\mathrm{kg} / \mathrm{m}^{2}, \mathrm{PSG}=$ polysomnography, $\mathrm{RDI}=$ respiratory disturbance index, $\mathrm{ODI}=$ oxygen desaturation index .

\subsubsection{The Impact of Sleep-Disordered Breathing on Short-Term Indices of Fetal Wellbeing - Cardiotocography}

\subsubsection{Overall Summary of Cardiotocography Results}

Overall, we had at least $50 \%$ of total sleep time (TST) with successful fetal heart rate tracing on the CTG for 67 out of $82(81.7 \%)$ studies, and at least $75 \%$ of TST with successful fetal heart rate trace on $54(65.9 \%)$ studies. A total of 59 studies $(72.0 \%)$ met our inclusion criteria for analysis, with CTG monitoring being successful on more control and $\mathrm{GH}$ compared to PE participants (control $=76.2 \%$, $\mathrm{GH}=87 \%$ and $\mathrm{PE}=41.2 \%, \mathrm{p}=.004)$. CTG monitoring success was unrelated to $\mathrm{BMI}(r=.03, p=.78)$ or severity of SDB (RDI $\log r=.08, p=.49)$, but was related to gestational age $(r=.57, p<.001)$ which was likely a contributor towards the lower success rate in the PE group who had PSG performed earlier in gestation (Supplementary Table 5.10). 


\subsubsection{Indicators of Fetal Distress on Cardiotocography}

\subsection{Impact of Sleep-Disordered Breathing Overall}

Table 5.2 details the characteristics of those with a successful CTG recording, for the SDB versus No SDB groups. There were no differences in gestation at which the CTG was performed, nor measures of CTG success during sleep.

Table 5.2 Cardiotocography Variables for SDB vs No SDB Groups

\begin{tabular}{|c|c|c|c|}
\hline & $\begin{array}{c}\text { SDB } \\
(n=29)\end{array}$ & $\begin{array}{l}\text { No SDB } \\
(n=30)\end{array}$ & $\mathrm{p}$ \\
\hline \% CTG success & $92.1 \pm 10.9$ & $93.9 \pm 9.0$ & .49 \\
\hline Hours of CTG success & $5.8 \pm 1.4$ & $6.1 \pm 1.0$ & .24 \\
\hline Gestation CTG (weeks) & $34.3 \pm 2.8$ & $34.1 \pm 2.3$ & .82 \\
\hline $\mathrm{BMI}$ at $\mathrm{CTG}$ & $38.0 \pm 6.7$ & $34.9 \pm 5.4$ & .054 \\
\hline $\mathrm{RDI} / \mathrm{hr}$ & $12.3(7.4,30.9)$ & $2.9(1.3,3.9)$ & $<.001$ \\
\hline \multicolumn{4}{|l|}{ Any event } \\
\hline$n(\%)$ & $11(37.9 \%)$ & $9(30.0 \%)$ & .59 \\
\hline Total events/night* & $2.4 \pm 1.0$ & $2.3 \pm 1.4$ & .96 \\
\hline \multicolumn{4}{|l|}{ Event 1} \\
\hline n (\%) & $1(3.4 \%)$ & $2(6.7 \%)$ & 1.0 \\
\hline \multicolumn{4}{|l|}{ Event 2} \\
\hline n (\%) & $0(0.0 \%)$ & $0(0.0 \%)$ & - \\
\hline \multicolumn{4}{|l|}{ Event 3} \\
\hline n (\%) & $0(0.0 \%)$ & $0(0.0 \%)$ & - \\
\hline \multicolumn{4}{|l|}{ Event 4} \\
\hline $\mathrm{n}(\%)$ & $11(37.9 \%)$ & $9(30.0 \%)$ & .59 \\
\hline
\end{tabular}

Note. Values given as $\mathrm{M} \pm \mathrm{SD}$, Mdn (IQR) or $\mathrm{n}(\%)$. SDB = sleep-disordered breathing, CTG $=$ cardiotocography, $\mathrm{BMI}=$ body mass index $\mathrm{kg} / \mathrm{m}^{2}, \mathrm{RDI}=$ respiratory disturbance index.

*only those who had fetal heart rate events.

Event 1 = Prolonged bradycardia ( $>15 \mathrm{bpm}$ fall below baseline for $\geq 90 \mathrm{sec}$ and $<5 \mathrm{~min}$ ), Event $2=$ Recurrent severe variable decelerations ( $>60 \mathrm{bpm}$ fall from baseline, $>60 \mathrm{sec}$ and $\geq 2$ per $2 \mathrm{hrs}$, Event $3=$ Repeated unprovoked decelerations or late decelerations, Event $4=$ deceleration lasting between $60-90 \mathrm{sec}$ with $>15 \mathrm{bpm}$ fall from baseline.

Of all the participants, $20(33.9 \%)$ of the CTGs showed at least one of the four events potentially indicating an episode of fetal compromise. The number of overall CTG events overnight per participant ranged from $0-5$. There were no episodes of the more severe indicators of fetal compromise - Event 
2 - recurrent severe variable decelerations or Event 3 - repeated unprovoked decelerations or late decelerations, on any of the fetal heart rate recordings. There was no impact of SDB on the number of participants displaying Event 1 or Event 4 (prolonged bradycardia or 60-90 sec fetal heart rate decelerations), with very few fetuses experiencing bradycardia (Event 1) overnight. There was also no difference in the number of events per night in those who displayed fetal heart rate events among those with and without SDB (Table 5.2).

\subsection{Impact of Sleep-Disordered Breathing in Hypertensive Disorders of Pregnancy and Control Groups}

Table 5.3 details the characteristics of those with a successful CTG recording, with GH and PE groups combined into HDP. Again, there were no differences between those with and without SDB, apart from $\mathrm{RDI} / \mathrm{hr}$. Within the HDP and control groups, SDB again had no impact on the number of participants displaying Event 1 or Event 4 (prolonged bradycardia or 60-90 sec fetal heart rate decelerations), and among those who displayed fetal heart rate events, there were no differences in the total number of events overnight between those with or without SDB within the hypertensive and normotensive group (Table 5.3). Illustrative examples of the concurrent CTG and PSG recordings are given in Figures 5.3 and 5.4 . 
Table 5.3 Cardiotocography Variables for Hypertensive Disorders of Pregnancy Cases and Normotensive Controls stratified by $S D B$

\begin{tabular}{lcccccc}
\hline & \multicolumn{2}{c}{$\begin{array}{c}\text { HDP } \\
\end{array}$} & \multicolumn{2}{c}{ S $n=27)$} & \multicolumn{3}{c}{$\begin{array}{c}\text { Controls } \\
(\mathrm{n}=32)\end{array}$} \\
\cline { 2 - 7 } & $\begin{array}{c}\text { SDB } \\
(\mathrm{n}=17)\end{array}$ & $\begin{array}{c}\text { No SDB } \\
(\mathrm{n}=10)\end{array}$ & $\mathrm{p}$ & $\begin{array}{c}\text { SDB } \\
(\mathrm{n}=12)\end{array}$ & $\begin{array}{c}\text { No SDB } \\
(\mathrm{n}=20)\end{array}$ & $\mathrm{p}$ \\
\hline \% CTG success & $94.3 \pm 8.0$ & $94.1 \pm 11.0$ & .95 & $88.9 \pm 13.9$ & $93.8 \pm 8.2$ & .22 \\
Hours of CTG & $5.7 \pm 1.6$ & $6.3 \pm 0.8$ & .25 & $5.8 \pm 1.3$ & $6.1 \pm 1.1$ & .50 \\
Gestation CTG & $34.7 \pm 3.1$ & $35.3 \pm 2.7$ & .64 & $33.6 \pm 2.3$ & $33.5 \pm 1.9$ & .93 \\
BMI at CTG & $37.4 \pm 6.5$ & $35.4 \pm 5.5$ & .41 & $38.8 \pm 7.2$ & $34.7 \pm 5.4$ & .07 \\
RDI/hr & $14.3(7.1$, & $2.7(1.1,3.8)$ & $<.001$ & $8.7(7.5,38.4)$ & $2.9(1.4,4.0)$ & $<.001$ \\
& $30.9)$ & & & & & \\
\hline
\end{tabular}

\begin{tabular}{lcccccc}
\hline Any event & & & & & \\
$\mathrm{n}(\%)$ & $6(35.3 \%)$ & $4(40.0 \%)$ & 1.0 & $5(41.7 \%)$ & $5(25.0 \%)$ & .44 \\
$\begin{array}{l}\text { Total events/ } \\
\text { night }\end{array}$ & $2.7 \pm 1.0$ & $2.8 \pm 2.1$ & .94 & $2.0 \pm 1.0$ & $2.0 \pm 0.7$ & 1.0 \\
$\begin{array}{l}\text { Event } 1 \\
\text { n (\%) }\end{array}$ & $1(5.9 \%)$ & $1(10.0 \%)$ & 1.0 & $0(0.0 \%)$ & $1(5.0 \%)$ & 1.0
\end{tabular}

Event 4

$\begin{array}{lllllll}\mathrm{n}(\%) & 6(35.3 \%) & 4(40.0 \%) & 1.0 & 5(41.7 \%) & 5(25.0 \%) & .44\end{array}$

Note. Values given as $\mathrm{M} \pm \mathrm{SD}$, Mdn (IQR) or $\mathrm{n}(\%)$. SDB = sleep-disordered breathing, HDP $=$ hypertensive disorders of pregnancy, $\mathrm{CTG}=$ cardiotocography, $\mathrm{BMI}=$ body mass index $\mathrm{kg} / \mathrm{m}^{2}, \mathrm{RDI}=$ respiratory disturbance index. Event 2 and 3 omitted from this table due to no events.

*only those who had fetal heart rate events.

Event 1 = Prolonged bradycardia ( $>15 \mathrm{bpm}$ fall below baseline for $\geq 90 \mathrm{sec}$ and $<5 \mathrm{~min}$ ), Event $4=$ deceleration lasting between $60-90 \mathrm{sec}$ with $>15 \mathrm{bpm}$ fall from baseline. 


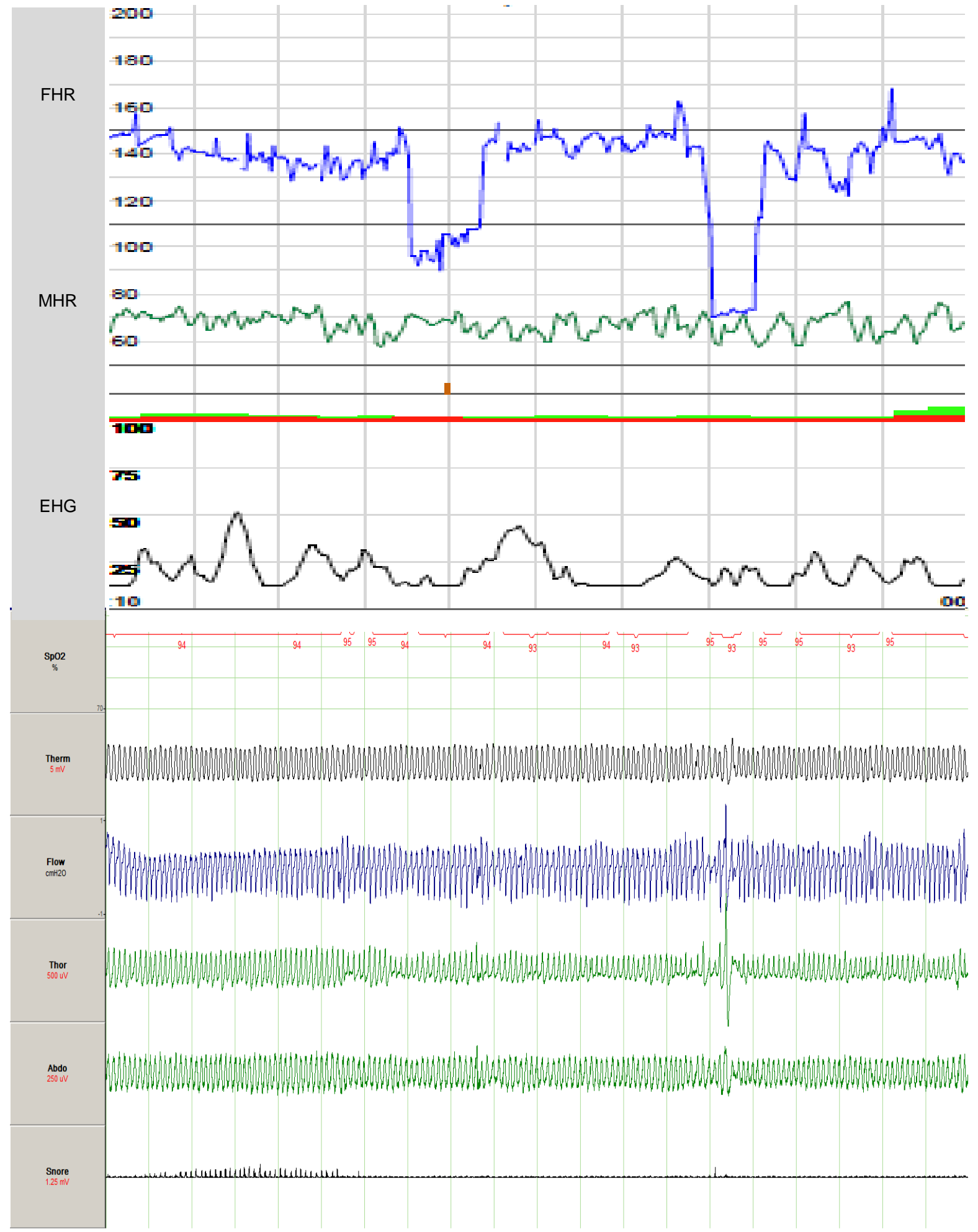

Figure 5.3 Time-synchronised cardiotocography (CTG) with polysomnography (PSG) across a 10 minute period of NREM sleep. This patient had preeclampsia (PE) with a well-grown fetus and was $34+1$ weeks gestation. Two fetal heart rate decelerations were observed during this period, but were unrelated to SDB events. The top panel shows the fetal heart rate $(F H R)$, maternal heart rate $(M H R)$ and uterine activity (electro hysterogram $=E H G)$. The bottom panel shows signals related to maternal respiration, including SpO2 (arterial oxygen saturation), Therm = thermocouple, Flow $=$ nasal airflow, Thor $=$ thoracic respiratory effort, Abdo = abdominal respiratory effort, and Snore. 


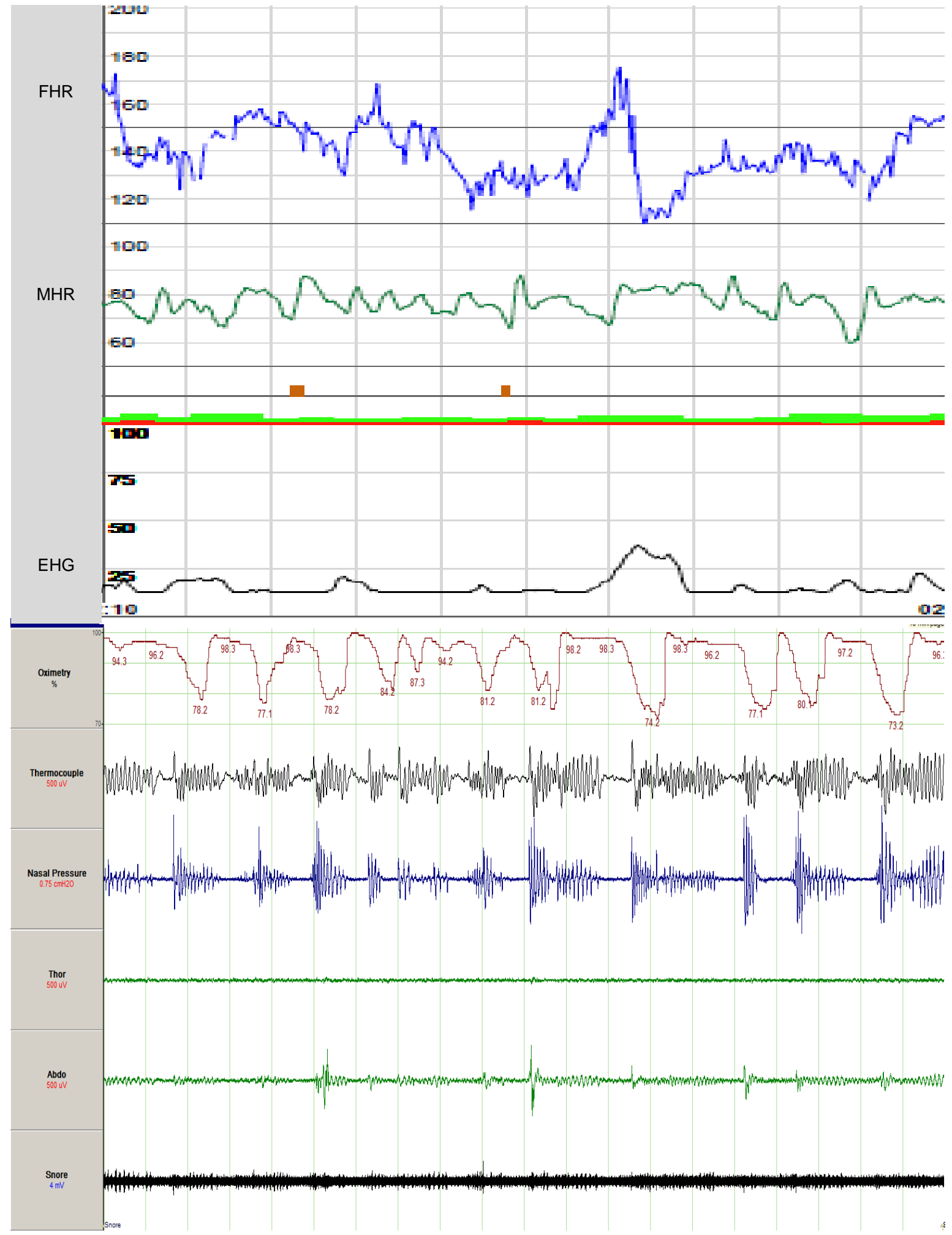

Figure 5.4 Time-synchronised cardiotocography (CTG) with polysomnography (PSG) across a 10 minute period of REM sleep. This patient had preeclampsia (PE) with a well-grown fetus and was 34+3 weeks gestation. Despite significant SDB (RDI of 39.7 overall) with oxygen desaturation down to a nadir of $73 \%$, the CTG did not demonstrate any pathological fetal heart rate responses overnight. The top panel shows the fetal heart rate (FHR), maternal heart rate $(M H R)$ and uterine activity (electro hysterogram $=E H G)$. The bottom panel shows signals related to maternal respiration, including Oximetry (arterial oxygen saturation), Thermocouple, Nasal Pressure, Thor $=$ thoracic respiratory effort (poor signal), Abdo = abdominal respiratory effort, and Snore 


\subsection{Multivariate Analysis of Risk Factors for Fetal Distress on Cardiotocography}

The number of overall CTG events per participant ranged from $0-5$; eight participants had an average of more than one deceleration event every 2 hours (rate of $>0.5$ per hour), 12 participants had decelerations but fewer than once every 2 hours ( $>0$ to 0.5 events per hour), with the remaining 39 participants having no events overnight.

When all relevant demographic variables were subject to univariate ordinal regression analysis, the factors associated with an increased risk of fetal heart rate deceleration events on CTG were nulliparity, earlier gestational age at CTG recording, diagnosis of PE and FGR at birth (Table 5.4). Neither presence nor severity of SDB in terms of RDI and ODI were individually associated with the number of events indicating fetal distress on nocturnal CTG.

Table 5.4 Ordinal Regression - Univariate Relationships Between Demographic Variables and Number of Fetal Heart Rate Events per Hour on Cardiotocography

\begin{tabular}{lccc}
\hline Variable & $\begin{array}{c}\text { Units or Category } \\
(\text { Range/SD })\end{array}$ & OR $(95 \%$ Cl $)$ & $\mathrm{p}$ \\
\hline Age & $\mathrm{yr}(21-40 / 4.4)$ & $1.04(0.61-1.78)$ & .89 \\
Nulliparous & Yes or No & $4.79(1.38-16.61)$ & .014 \\
BMI at CTG* & $\mathrm{kg} / \mathrm{m}^{2}(22.7-53.1 / 6.2)$ & $0.72(0.41-1.28)$ & .27 \\
Gestation at CTG* & weeks $(26.7-37.7 / 2.6)$ & $0.56(0.33-0.96)$ & .036 \\
\hline Antihypertensives & Yes or No & $2.07(0.62-6.95)$ & .24 \\
HDP & & & \\
GH vs. control & Yes or No & $1.00(0.30-3.33)$ & 1.0 \\
PE vs. control & Yes or No & $6.79(1.37-33.75)$ & .019 \\
FGR at birth & Yes or No & $4.58(1.36-15.46)$ & .014 \\
RDI $\geq 5$ & Yes or No & $1.49(0.52-4.31)$ & .46 \\
RDI log & Index $(-0.69-4.69 / 1.22)$ & $1.45(0.85-2.47)$ & .17 \\
ODI $\geq 3 \%$ log* & Index (-4.61 $-4.67 / 2.51)$ & $1.26(0.72-2.20)$ & .40
\end{tabular}

Note. $\mathrm{N}=59 . \mathrm{OR}=$ odds ratio, $\mathrm{BMI}=$ body mass index, $\mathrm{CTG}=$ cardiotocography, $\mathrm{HDP}=$ hypertensive disorders of pregnancy, $\mathrm{GH}=$ gestational hypertension, $\mathrm{PE}=$ preeclampsia, $\mathrm{FGR}=$ fetal growth restriction, $\mathrm{RDI}=$ respiratory disturbance index, $\mathrm{ODI}=$ oxygen desaturation index .

*OR for continuous variables indicate the change in odds for an increase of one standard deviation.

A stepwise selection model with all explanatory variables with an $\alpha$ of less than 0.20 on univariate analysis was performed. Given that GH had no association with fetal heart rate decelerations when 
compared with controls whereas PE was significantly related, a new variable was created comparing the control and GH women to the PE participants. This variable was not significant $(p=.28)$ and did not improve the model at all and was therefore removed. Information on how GH and PE impacted on the CTG results is shown in Supplementary Table 5.10.

The final model confirmed FGR at birth as the strongest significant predictor of number of fetal heart rate events per hour on CTG $\left(X^{2}=18.63, p=.001, R^{2}=0.33\right.$; Table 5.5). Nulliparity remained significantly associated with an increase in fetal heart rate decelerations, and severity of SDB in terms of overall RDI also became significant in the multivariate model.

Table 5.5 Factors associated with Number of Fetal Heart Rate Events per hour on Cardiotocography on Stepwise Ordinal Regression Modelling

\begin{tabular}{|c|c|c|c|c|}
\hline Variable & $B$ & Wald $x^{2}$ test & $\mathrm{aOR}(95 \% \mathrm{Cl})$ & $\mathrm{p}$ \\
\hline Gestation at CTG* & -0.22 & 2.94 & $0.57(0.30-1.08)$ & .09 \\
\hline Nulliparous & 1.51 & 5.02 & $4.53(1.21-17.01)$ & .03 \\
\hline FGR at birth & 1.67 & 5.20 & $5.31(1.26-22.26)$ & .02 \\
\hline RDI log* & 0.62 & 4.51 & $2.12(1.06-4.24)$ & .03 \\
\hline Threshold coefficient $=0$ & -5.77 & 1.77 & & \\
\hline Threshold coefficient $=1$ & -4.22 & 0.96 & & \\
\hline
\end{tabular}

Note. $\mathrm{N}=59$. aOR = adjusted odds ratio, $\mathrm{CTG}=$ cardiotocography, FGR = fetal growth restriction, RDI $=$ respiratory disturbance index.

*OR for continuous variables indicate the change in odds for an increase of one standard deviation.

Threshold coefficient $=0$ - the odds of FHR events per hour being 0 .

Threshold coefficient $=1$ - the odds of FHR events per hour being 0.5 per hour or less.

\subsection{Severity of Sleep-Disordered Breathing and Fetal Distress on Cardiotocography}

As shown in Table 5.5, severity of SDB in terms of RDI (log transformed) was significantly associated with the number of fetal heart rate events per hour on nocturnal CTG after adjustment for gestational age, nulliparity and FGR at birth. This relationship was strongest within the control group $(r=.44, p=$ $.02, n=28)$ but was not present within the HDP group $(r=.21, p=.40, n=18)$. As demonstrated in Supplementary Figure 5.7, there were no fetal heart rate events observed in control participants with an RDI (log transformed) of less than 1.2, which corresponded to an RDI of $<3.5(n=9)$. In comparison, 8 out of $19(42.1 \%)$ control participants with an RDI $\geq 3.5$ demonstrated fetal heart rate events on CTG overnight $(p=.03)$. 


\subsection{Relationship between Fetal Growth Restriction and Cardiotocography}

As shown in Table 5.5 above, FGR at birth was associated with the number of fetal heart rate events per hour on nocturnal CTG. Specifically, over $60 \%$ of mothers with a growth restricted infant displayed evidence of CTG abnormalities during sleep, versus only $26 \%$ of well-grown fetuses $(p=.04$, Table $5.6)$.

We considered whether respiratory events during sleep may exacerbate, or precipitate, these fetal heart rate events in FGR fetuses. Of the 13 participants with FGR neonates, six of them had SDB but it should be noted that these cases of SDB were all mild. Given this, and the small numbers in each group, it is perhaps unsurprising that we did not observe any differences in CTG abnormalities among women with SDB compared to those without (proportion of participants with any fetal event - SDB $=50.0 \%$ vs. No $\mathrm{SDB}=71.4 \%, p=.91 ;$ number of events overnight $-\mathrm{SDB}=2.7 \pm 1.5$ vs. No SDB $=2.8 \pm 1.6, p=.23$ ).

Table 5.6 Relationship between Fetal Heart Rate Events on Cardiotocography and Fetal Growth

\begin{tabular}{lccc}
\hline & $\begin{array}{c}\text { FGR at birth } \\
(\mathrm{n}=13)\end{array}$ & $\begin{array}{c}\text { Well-Grown } \\
(\mathrm{n}=46)\end{array}$ & $\mathrm{p}$ \\
\hline None & $5(38.5 \%)$ & $34(73.9 \%)$ & .04 \\
$>0$ to 0.5 events $\mathrm{h} / \mathrm{h}$ & $4(30.8 \%)$ & $8(17.4 \%)$ & \\
rate of $>0.5 \mathrm{p} / \mathrm{h}$ & $4(30.8 \%)$ & $4(8.7 \%)$ & \\
\hline
\end{tabular}

Note. Values given as (\%). FGR = fetal growth restriction, $\mathrm{p} / \mathrm{h}=$ per hour.

\subsubsection{The Impact of Sleep-Disordered Breathing on Long-Term Indices of Fetal Wellbeing - Fetal Growth and Perinatal Outcomes}

As described in Section 5.2.4 Statistical Analysis page 135, only participants who had a third trimester ultrasound performed at least 2 weeks prior to delivery were included in the analysis of slowed third trimester growth. Separate data is shown for birthweight indices for all participants (Table 5.7 and 5.8). The third trimester ultrasounds were performed between $28+1$ and $37+2$ weeks of gestation, at an average of $33+1$ weeks.

\subsubsection{Fetal Growth and Sleep-Disordered Breathing}

Measures of fetal growth including FGR at birth and third trimester growth trajectory for those with and without SDB are given in Table 5.7. The presence of SDB did not significantly impact on birthweight centile or FGR at birth, nor did it contribute to slowed third trimester growth.

When analysis was confined to those with an RDI $\geq 15$ (Supplementary Table 5.11), there was evidence that infants of mothers with SDB in fact had a larger birthweight centile and less likelihood of being FGR 
at birth, compared to infants of mothers with an $\mathrm{RDI}<15$. This relationship with birthweight centile remained $(p=.07)$ after controlling for the slightly higher proportion of women with pre-existing FGR in the No SDB group at the time of recruitment. Moderate to severe SDB did not have any impact on fetal growth or growth trajectory in the third trimester.

Table 5.7 Measures of Fetal Growth in the SDB vs No SDB groups

\begin{tabular}{|c|c|c|c|}
\hline & SDB & No SDB & $p$ \\
\hline All participants & $n=34^{*}$ & $n=45$ & \\
\hline $\mathrm{RDI} / \mathrm{hr}$ & $12.2(7.4,24.8)$ & $2.4(1.3,3.7)$ & $<.001$ \\
\hline Birth gestation (weeks) & $37.6 \pm 3.5$ & $37.6 \pm 3.5$ & .99 \\
\hline Birthweight (g) & $3115.7 \pm 993.4$ & $2989.5 \pm 1000.8$ & .58 \\
\hline Birthweight cust. centile (\%) & $42.6 \pm 34.3$ & $32.4 \pm 30.1$ & .16 \\
\hline FGR at recruitment & $4(11.8 \%)$ & $6(13.3 \%)$ & 1.0 \\
\hline FGR at birth ( $<10^{\text {th }}$ cust. centile $)$ & $8(23.5 \%)$ & $17(37.8 \%)$ & .23 \\
\hline $\begin{array}{l}\text { Impaired fetal growth (FGR or } \\
\text { fall in cust. centile }>33 \% \text { ) }\end{array}$ & $15(44.1 \%)$ & $21(46.7 \%)$ & 1.0 \\
\hline Participants with valid T3 scan & $n=29^{*}$ & $n=34$ & \\
\hline $\mathrm{RDI} / \mathrm{hr}$ & $12.0(7.5,25.3)$ & $2.3(1.3,3.7)$ & $<.001$ \\
\hline T3 US gestation (weeks) & $33.3 \pm 1.6$ & $33.1 \pm 1.8$ & .69 \\
\hline T3 cust. centile (\%) & $56.4 \pm 29.7$ & $52.2 \pm 27.4$ & .56 \\
\hline Birth gestation (weeks) & $38.7 \pm 1.6$ & $38.8 \pm 2.1$ & .88 \\
\hline Birthweight (g) & $3377.1 \pm 595.2$ & $3328.9 \pm 728.4$ & .78 \\
\hline Birthweight cust. centile (\%) & $46.6 \pm 32.0$ & $40.0 \pm 29.8$ & .40 \\
\hline Change in cust. centile, total (\%) & $-10.0 \pm 29.0$ & $-12.1 \pm 19.0$ & .73 \\
\hline $\begin{array}{l}\text { Change in cust. centile per day } \\
(\%)\end{array}$ & $-0.44 \pm 1.0$ & $-0.39 \pm 0.7$ & .85 \\
\hline $\begin{array}{l}\text { Fall in cust. centile }>33 \% \text { over } \\
6 \text { weeks }\end{array}$ & $8(27.6 \%)$ & $6(17.6 \%)$ & .38 \\
\hline \multicolumn{4}{|c|}{$\begin{array}{l}\text { Note. Values given as } \mathrm{M} \pm \mathrm{SD}, \mathrm{Mdn}(\mathrm{IQR}) \text {, or } \mathrm{n}(\%) . \mathrm{SDB}=\text { sleep-disordered breathing, } \mathrm{RDI}= \\
\text { respiratory disturbance index, cust. = customised, } \mathrm{FGR}=\text { fetal growth restriction, } \mathrm{T} 3=\text { third trimester, } \\
\text { US = ultrasound. } \\
{ }^{*} \text { excludes } 3 \mathrm{CPAP} \text { users. }\end{array}$} \\
\hline
\end{tabular}




\subsubsection{Fetal Growth and Perinatal Outcomes - Impact of Sleep-Disordered Breathing in Hypertensive Disorders of Pregnancy and Control Groups}

\subsection{Hypertensive Disorders of Pregnancy Group}

Among the HDP participants, there was no difference in gestational age at birth for those with and without SDB. The infants of women with SDB were significantly larger at birth than those without SDB in terms of birthweight customised centile and presence of FGR at birth (Table 5.8). There was a higher proportion of PE participants with FGR at birth in the No SDB group (Supplementary Table 5.12) compared to those with SDB, however the association between SDB and larger infants persisted even after adjustment for the proportion of PE participants in each SDB group $(p=.052)$.

Fetal growth trajectory from the third trimester ultrasound to birth for those with and without SDB is shown in Figure 5.5. As expected, the fall in customised centile per day was greater amongst the HDP women (particularly PE; Table 5.13) than controls, reflecting their underlying placental dysfunction. However, the presence or absence of SDB did not significantly impact further among women with HDP (change in customised centile per day $-\mathrm{SDB}=-0.71 \pm 1.35$ vs. No SDB $=-0.74 \pm 1.22, p=.96$ ). There was no difference in the number of fetuses with slowed third trimester growth $(>33 \%$ fall in customised centile; $40.0 \%$ vs. $33.3 \%, p=1.0$ ).

In terms of perinatal outcomes (see Table 5.8), the number of preterm births ( $<37$ weeks of gestation) in the No SDB group tended to be higher than in the SDB group within those with HDP. Again, this is likely influenced by the higher rate of PE in the No SDB group. Caesarean delivery, including those done for emergency reasons, was very common in HDP regardless of presence of SDB. In terms of health at birth, a higher proportion of infants with mothers with SDB had reduced Apgar scores however these differences were not significant. Neonatal admission to NICU or SCN was not associated with SDB in the mother.

Confining analysis to those with moderate-to-severe SDB (RDI $\geq 15)$ did not reveal any evidence of compromised perinatal outcomes such as mode of delivery, Apgar scores or NICU admission (Supplementary Table 5.11).

\subsection{Control Group}

For control participants, there were no differences in birthweight, customised birthweight centile, gestational age at birth, or rate of FGR at birth between the SDB and No SDB groups (Table 5.8). Fetal growth trajectory from the third trimester ultrasound to birth for those with and without SDB is shown in Figure 5.5. There were no differences in customised centiles for fetal weight in the third trimester or at birth between the SDB and No SDB groups ( $p=.51$ and $p=.83$ respectively). The magnitude of the drop in fetal growth centile per day was smaller than within the PE group (see Supplementary Table 5.13), but again this was not influenced by presence of SDB (SDB $=-0.15 \pm 0.45$ vs. No SDB $=-0.27 \pm$ 
$0.44, p=.42$ ). There were no differences in the number of fetuses whose growth centile was projected to fall by $>33 \%$ from the third trimester to birth $(S D B=14.3 \%$ vs. No SDB $=12.0 \%, p=1.0$ ).

For perinatal outcomes, control participants all gave birth at term (Table 5.8). Significantly more infants of mothers with SDB were admitted to SCN, however $2 / 3$ of these mothers also had GDM and the neonate was admitted briefly for observation. Low Apgar scores tended to be more common in the SDB group but this was not significant.

\subsection{Impact of Hypertensive Disease on Fetal Growth and Perinatal Outcomes}

See Supplementary Table 5.12 for a separate comparison of fetal growth indices for the GH and PE groups by SDB status, and Supplementary Table 5.13 for a comparison of fetal growth indices across the PE, GH and Control groups. As expected, several birth outcomes were found to be negatively affected by the presence of a hypertensive disorder (Supplementary Table 5.14), regardless of SDB status (see Supplementary Table 5.15 for SDB results for the GH and PE group separately). 
Table 5.8 Measures of Fetal Growth and Perinatal Outcomes for those with and without SDB in the Hypertensive Disorders of Pregnancy and Control Groups

\begin{tabular}{|c|c|c|c|c|c|c|}
\hline & \multicolumn{2}{|c|}{$\begin{array}{c}\text { HDP } \\
\left(n=39^{*}\right)\end{array}$} & \multicolumn{4}{|c|}{$\begin{array}{l}\text { Controls } \\
\left(n=40^{\star}\right)\end{array}$} \\
\hline & $\begin{array}{c}\text { SDB } \\
(n=20)\end{array}$ & $\begin{array}{l}\text { No SDB } \\
(n=19)\end{array}$ & $\mathrm{p}$ & $\begin{array}{c}\text { SDB } \\
(n=14)\end{array}$ & $\begin{array}{l}\text { No SDB } \\
(n=26)\end{array}$ & $\mathrm{p}$ \\
\hline Birth Gestation (weeks) & $36.3 \pm 4.0$ & $34.9 \pm 3.8$ & .26 & $39.4 \pm 1.3$ & $39.6 \pm 1.1$ & .69 \\
\hline Birthweight (g) & $2881.6 \pm 1188.6$ & $2223.9 \pm 1012.9$ & .07 & $3450.2 \pm 485.3$ & $3549.0 \pm 503.2$ & .55 \\
\hline Birthweight cust. centile (\%) & $43.2 \pm 38.3$ & $16.2 \pm 27.0$ & .015 & $41.9 \pm 29.0$ & $44.3 \pm 26.9$ & .79 \\
\hline FGR at recruitment & $4(20.0 \%)$ & $6(31.6 \%)$ & .48 & $0(0 \%)$ & $0(0 \%)$ & 1.0 \\
\hline FGR at birth $\left(<10^{\text {th }}\right.$ cust. centile) & $6(30.0 \%)$ & $13(68.4 \%)$ & .026 & $2(14.3 \%)$ & $4(15.4 \%)$ & 1.0 \\
\hline $\begin{array}{l}\text { Impaired fetal growth (FGR or } \\
\text { fall in cust. centile }>33 \% \text { ) }\end{array}$ & $11(55.0 \%)$ & 15 (78.9\%) & .18 & $4(28.6 \%)$ & $6(23.1 \%)$ & .72 \\
\hline Preterm Birth (<37 weeks) & $6(30.0 \%)$ & $12(63.2 \%)$ & .056 & $0(0 \%)$ & $0(0 \%)$ & - \\
\hline Caesarean \% & $15(75.0 \%)$ & $15(78.9 \%)$ & 1.0 & $6(42.9 \%)$ & $11(42.3 \%)$ & 1.0 \\
\hline Emergency Caesarean & $9(45.0 \%)$ & $10(52.6 \%)$ & .75 & $3(21.4 \%)$ & $5(19.2 \%)$ & 1.0 \\
\hline Apgar $1 \min \leq 7$ & $8(40.0 \%)$ & $4(21.1 \%)$ & .30 & $3(21.4 \%)$ & $2(8.0 \%)$ & .33 \\
\hline Apgar $5 \min \leq 7$ & $3(15.0 \%)$ & $0(0 \%)$ & .23 & $1(7.1 \%)$ & $0(0 \%)$ & .36 \\
\hline NICU admit & $3(15.0 \%)$ & $5(26.3 \%)$ & .45 & $0(0 \%)$ & $0(0 \%)$ & - \\
\hline SCN admit & $5(25.0 \%)$ & 7 (36.8\%) & .50 & 3 (21.4\%) & $0(0 \%)$ & .04 \\
\hline
\end{tabular}

Note. Values given as M \pm SD or $\mathrm{n}(\%)$. HDP = hypertensive disorders of pregnancy, SDB = sleep-disordered breathing, cust. = customised, FGR = fetal growth restriction, $\mathrm{NICU}=$ neonatal intensive care unit, $\mathrm{SCN}=$ special care nursery.

*excluding three participants commenced on CPAP ( 1 x HDP, $2 \times$ controls $)$ 


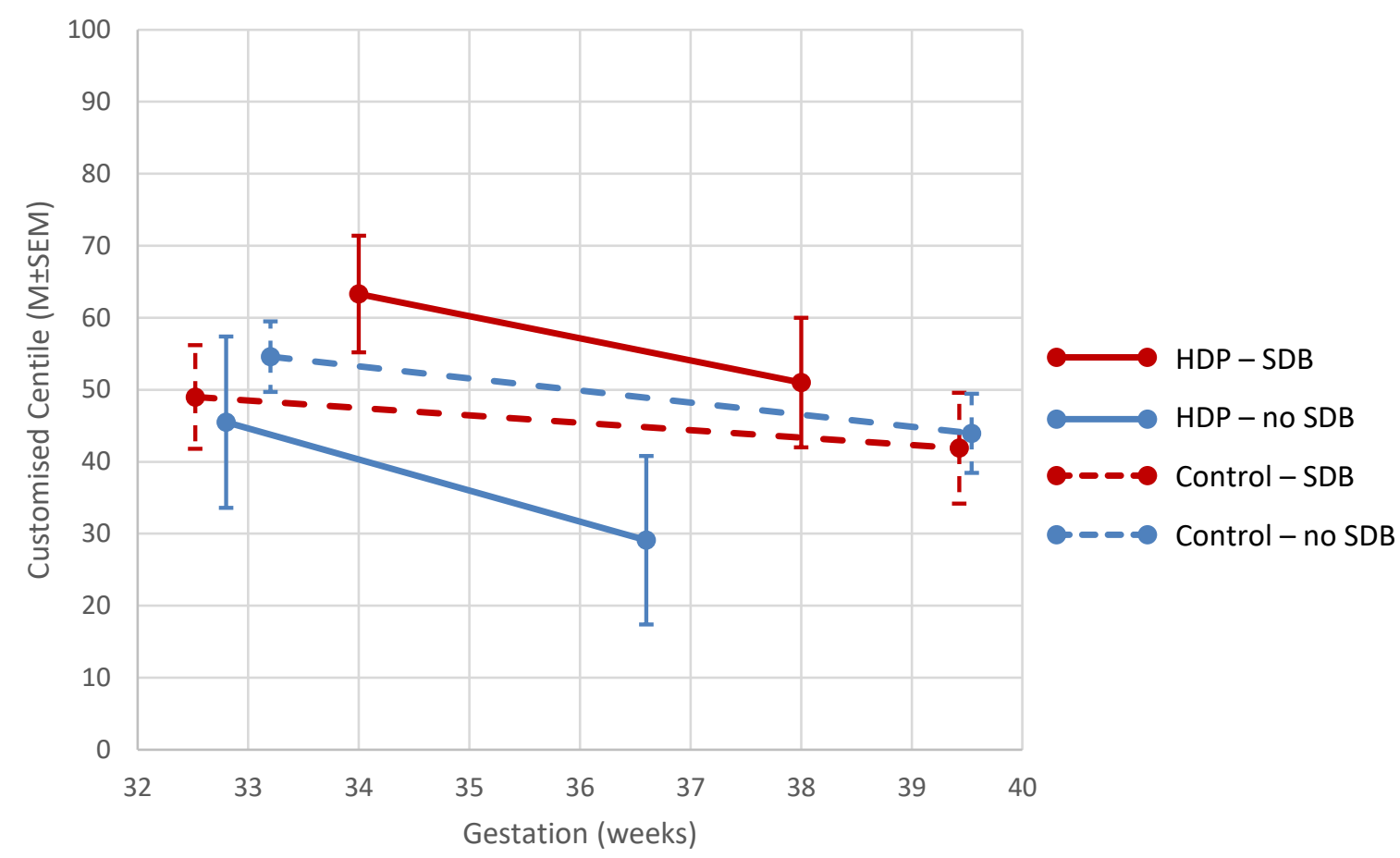

Figure 5.5 Growth trajectory between estimated fetal weight (EFW) from ultrasonography in the third trimester to birthweight using customised centiles. $H D P$ group $-S D B n=15$, no $S D B n=9$. Control group $S D B n=14$, no $S D B n=25$. $H D P=$ hypertensive disorders of pregnancy, $S D B=$ sleep-disordered breathing .

\subsubsection{The Impact of Sleep-Disordered Breathing on Long-Term Indices of Fetal Wellbeing - Biomarkers of Fetal Growth in Cord Blood}

Venous cord blood data was obtained for a total of 30 HDP participants and 27 controls. One control sample was excluded as it contained arterial rather than venous blood, and the IGF-2 value did not read for one control participant. The following data was excluded from analysis due to extreme outlying values ( $z$ scores $>3.3$ ) - IGF-1 = one control participant, IGFBP-1 = two controls and one PE participant, and IGFBP-2 = one control participant. The excluded control data was for a different participant for each IGF measure.

\subsubsection{Biomarkers of Fetal Growth - Impact of Sleep-Disordered Breathing in Hypertensive Disorders of Pregnancy and Control Groups}

The gestational age at which the cord blood sample was taken did not differ within the HDP group (SDB $-\mathrm{M} \pm \mathrm{SD}=36.6$ weeks \pm 3.8 vs No SDB $=35.2 \pm 4.2, \mathrm{p}=.34$ ) or the control group (SDB $-38.8 \pm 1.0$ vs No SDB $=39.4 \pm 1.2, p=.25)$. As shown in Figure 5.6 , there were no significant differences observed between the SDB and no SDB group for IGF-1 ( $p=.57)$, IGF-2 ( $p=.39)$, IGFBP-1 ( $p=.22)$ or IGFBP$2(p=.95)$ for the HDP participants. Individually, SDB had no impact on these insulin markers of fetal 
growth within the GH or PE groups either (Supplementary Table 5.16). However, in the control group, the participants with SDB had significantly higher IGF-1, IGF-2 and IGFBP-2 (Figure 5.6). There was no difference in IGFBP-1 $(p=.11)$.

Increasing the threshold for diagnosis of SDB to RDI $\geq 15$ did alter the results somewhat for the IGF levels in cord blood (Supplementary Table 5.11). As was found in the control group at RDI $\geq 5$, there was evidence for those with an RDI $\geq 15$ to have higher levels of IGF-2. Conversely, those with an RDI $\geq 15$ had lower levels of IGFBP-1.

\subsubsection{Biomarkers of Fetal Growth - Impact of Hypertensive Disease}

See Supplementary Table 5.17 for a comparison of biomarkers of fetal growth in cord blood across the $\mathrm{PE}, \mathrm{GH}$ and control groups. 
IGF-1

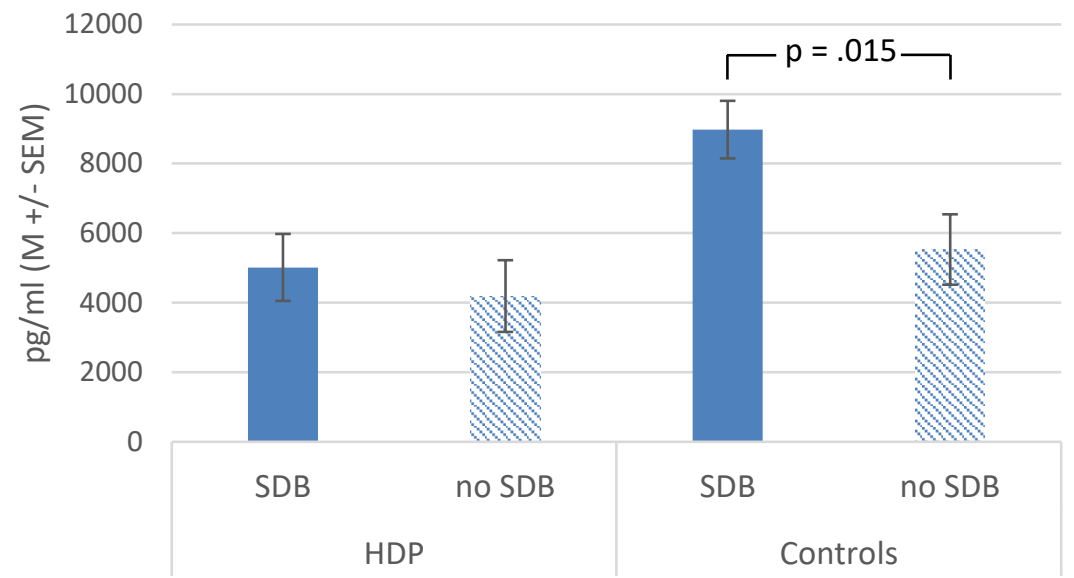

IGFBP-1

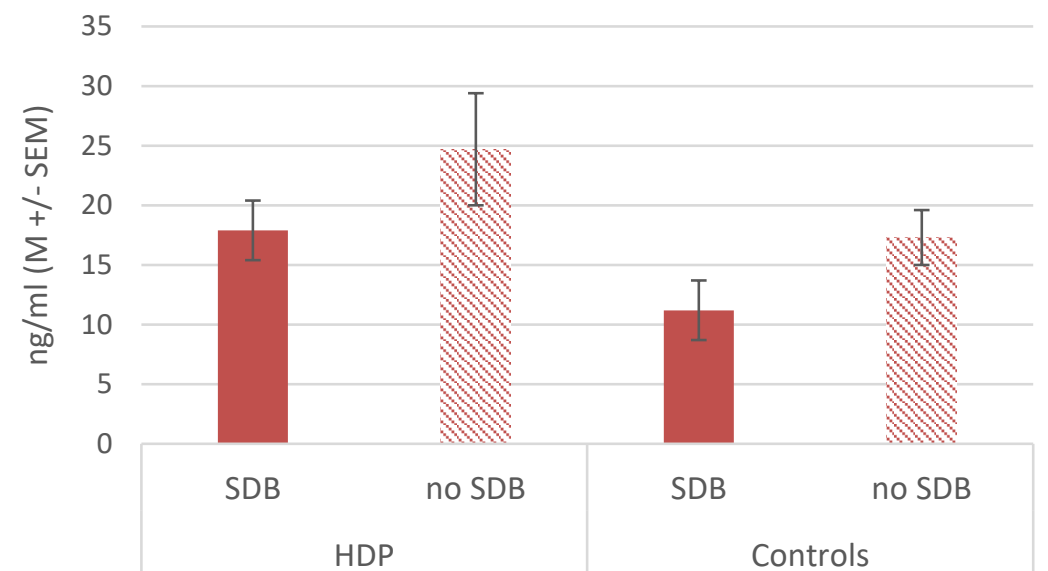

IGF-2

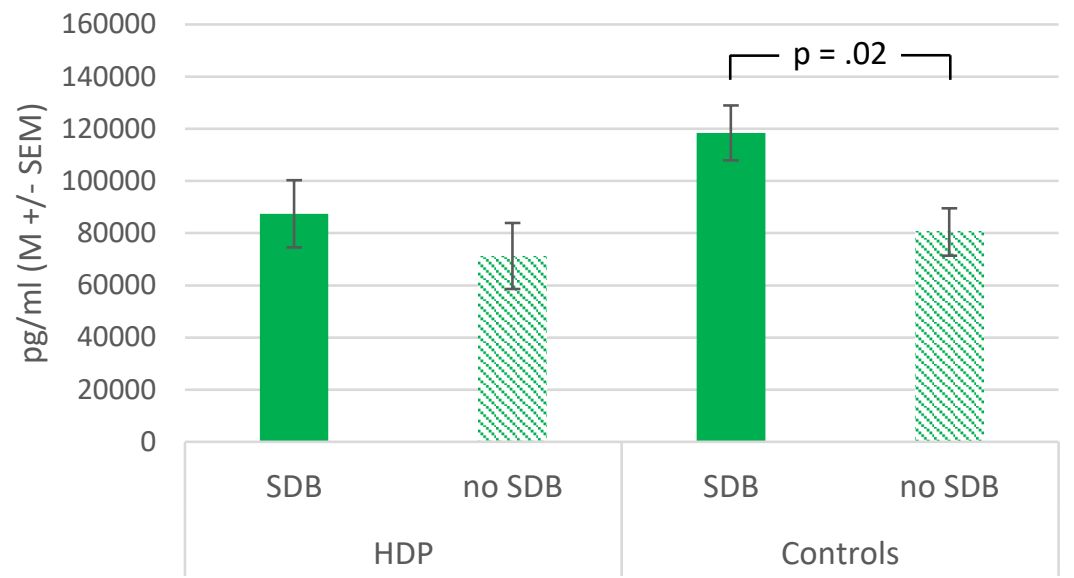

IGFBP-2

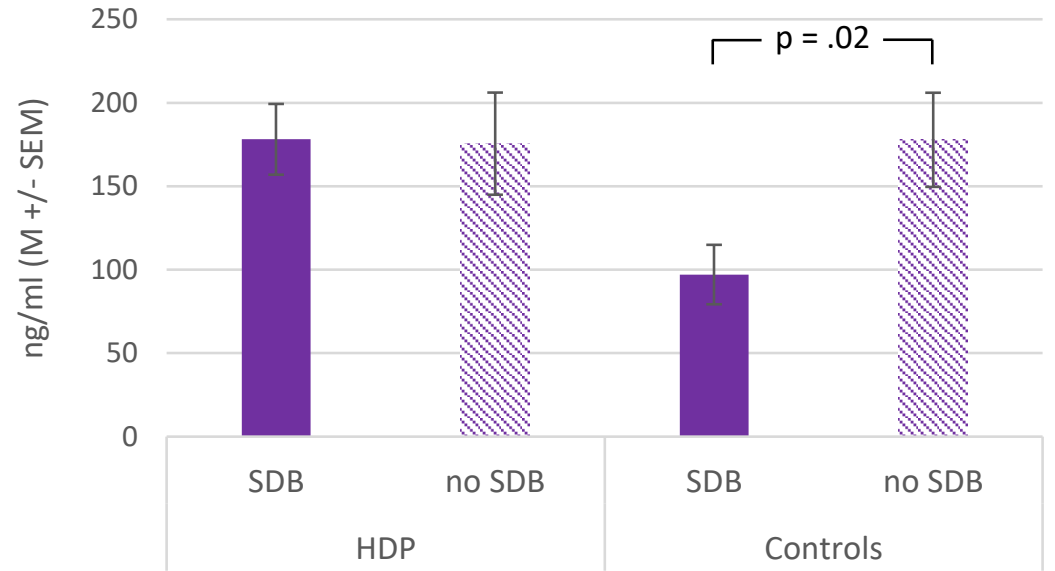

Figure 5.6 Insulin-like Growth Factor 1 and 2 (IGF-1, IGF-2) and Binding Protein 1 and 2 (IGFBP-1, IGFBP-2) for the SDB vs. No SDB group within the HDP and control group. $H D P$ group $-S D B n=17$, No $S D B n=13$. Control group $S D B n=8$, No $S D B n=17$, except for IGFBP-1 where HDP group - No $S D B n=12$, Control group No $S D B n=16$. $S D B=$ sleep-disordered breathing, $H D P=$ hypertensive disorders of pregnancy. 


\subsection{Discussion}

This chapter addressed whether fetal outcomes were worse in women with HDP and normotensive controls in the presence of SDB. We hypothesised that SDB would have a negative effect on nocturnal measures of fetal distress, fetal growth, perinatal outcomes and biomarkers of fetal growth in cord blood.

As a whole, our results show that women with HDP do not have poorer fetal outcomes when suffering from concurrent SDB. We did however observe some interesting results. Firstly, we found that FGR was strongly associated with fetal heart rate events on nocturnal CTG, whether growth restriction was already established during pregnancy or diagnosed at the time of birth. Secondly, we observed that SDB was independently associated with CTG abnormalities among well-grown control fetuses, and that these differences in fetal heart rate events were seen at a relatively lower diagnostic threshold for SDB $(R D I \geq 3.5$ rather than an RDI $\geq 5$ ). Thirdly, among women with HDP, fetal size at birth and the incidence of pre-term birth paradoxically appeared to be better among those with concurrent SDB. When analysis was confined to moderate to severe SDB, infants of mothers with SDB still showed evidence of larger birthweight centile and less likelihood of being FGR at birth compared to those with an RDI $<15$, mirroring some of the observations made in Chapter 4 - Maternal Outcomes.

\subsubsection{Acute Impact of SDB on Fetal Wellbeing - Cardiotocography}

We hypothesised that fetal well-being could be affected by maternal SDB leading to fetal hypoxia and fetal respiratory acidosis, particularly in already vulnerable fetuses such as those with mothers with a hypertensive disease of pregnancy. Maternal hypertension typically occurs as a result of excessive vasoconstriction, resulting in a reduced diameter of the uterine arteries - and therefore uterine blood flow - to meet the fetal metabolic requirements. In the setting of PE, chronic fetal hypoxia is more likely because poor placental development, from the beginning of pregnancy, leads to inadequate spiral arteriolar dilatation, reduced placental size and thus a reduced area available for gas and nutrient exchange. ${ }^{405}$ We proposed that the sympathetic activation and maternal hypoxaemia associated with nocturnal hypoxic events in SDB may amplify these effects, impacting on fetal oxygenation by reducing the availability of oxygen in the intervillous space, ${ }^{405}$ resulting in fetal distress being evident on time synchronised fetal heart rate monitoring.

A typical fetal response to acute hypoxia or acidosis is a sudden drop in heart rate, with the extent of the bradycardia depending on the degree of fetal hypoxia. ${ }^{251,406}$ Decelerations can be a normal physiological response to cord compression or occlusion associated with contractions in labour, or can occur when a fall in the level of oxygen in the fetal blood triggers chemoreceptors in the fetus to cause reflex constriction of blood vessels in nonvital peripheral areas in order to divert more blood flow to vital organs such as the adrenal glands, heart, and brain. The duration of the hypoxic insult and the intervals between insults are important - longer insults will generally cause greater hypoxia. While a moderate hypoxic insult is not necessarily a problem if it occurs infrequently, a mild insult that occurs every couple 
of minutes may be a problem, particularly in terms of depleting important reserves. ${ }^{405}$ This repetitive pattern of mild hypoxia may reflect the respiratory physiology associated with SDB.

Initially, our results found that the presence of SDB at an RDI $\geq 5$ had no impact on fetal well-being as assessed by nocturnal CTG monitoring. Rather, the strongest univariate relationships with fetal heart rate decelerations were a diagnosis of $P E$ and FGR at birth. This is unsurprising, given that PE and FGR are both associated with more severe degrees of underlying placental dysfunction. Multivariate analysis however showed that FGR was a more important predictor than PE, with the growth restricted babies at birth 5 times more likely to show fetal heart rate decelerations on nocturnal CTG in the third trimester. That FGR outstrips both PE and SDB as a risk factor for acute FHR changes during sleep is also not unexpected, given that FGR is so strongly associated with adverse perinatal outcome. Stillbirth rates in high-income countries such as Australia have shown little or no improvement over the past two decades, ${ }^{407}$ and approximately $50 \%$ of stillbirths are growth restricted. ${ }^{402,408}$ The risk of perinatal death is increased up to four times in FGR compared to appropriately grown babies, ${ }^{409,410}$ and FGR is a common cause of premature birth and intrapartum asphyxia. ${ }^{411}$ In particular, preterm infants with a birthweight centile of $<3 \%$ carry the highest perinatal morbidity and mortality risk. ${ }^{229}$ Thus, it might be expected that FGR fetuses have the least reserve to withstand further physiological insults at night.

For these important reasons, we had a particular interest in seeing whether the physiological events associated with maternal SDB would be more poorly tolerated by those fetuses already suffering uteroplacental insufficiency. A growth restricted fetus has little in the way of reserves to tolerate hypoxic insults, therefore even the mildest degree of oxygen deficiency may cause tissue hypoxia, asphyxial injury or even death in utero. ${ }^{412}$ In our study however, FGR fetuses were not further adversely impacted by maternal SDB in terms of nocturnal heart rate decelerations. This may be because FGR was not always severe at the time of the sleep study, and the SDB was generally mild. Examining fetal heart rate responses to sleep events in fetuses with severe FGR is part of our ongoing program of work.

To examine more closely the relationship between nocturnal fetal heart rate events and RDI, we analysed them as continuous, rather than dichotomous variables. After controlling for other variables, our study did suggest an association between nocturnal fetal heart rate events and RDI. Specifically, SDB at a lower threshold was most strongly associated with fetal heart rate events within the well-grown fetuses in the control group. Despite this, it should be kept in mind that the type of fetal heart rate events observed were only mild, with the two most 'severe' types of fetal heart rate decelerations not observed at all (recurrent severe variable decelerations and repeated unprovoked decelerations or late decelerations). The significance of milder variable decelerations depends greatly on the overall clinical picture and other features of the CTG, ${ }^{248}$ so these findings need to be interpreted with caution, and warrant further work.

The earliest study describing fetal heart rate changes in association with sleep apnoea was presented by Joel-Cohen and Schoenfeld, ${ }^{413}$ with a case study of an obese women at term whose fetus showed 
changes in baseline variability and decelerations on CTG during and after periods of witnessed apnoeas lasting between 70-80 seconds. Also supporting the potential link between maternal hypoxaemia and fetal distress was the first case study of confirmed OSA by PSG and fetal heart rate decelerations. ${ }^{241}$ Whilst an inpatient for PE with a growth-restricted fetus, a pregnant women was observed to have several apnoeic episodes during sleep. Auscultation of the fetal heart rate revealed a bpm of 90-100 during one episode of apnoea when maternal oxygen saturation had fallen to $80 \%$. A formal sleep study subsequently confirmed an $\mathrm{AHI}$ of $160 / \mathrm{hr}$. Subsequently, an early attempt at simultaneous PSG and CTG found that three out of four women diagnosed with OSA during pregnancy had fetal heart rate decelerations accompanying maternal oxygen desaturation, compared to only 1 of 31 pregnant women without OSA. ${ }^{240}$ However, this study was limited by the small number of women found to have OSA, who were mostly obese with the potential confounding variables of diabetes, hypertension and vascular disease.

Despite the theoretical implications of how maternal apnoea and hypoxaemia might impact on fetal heart rate events on CTG and the reported case studies, we did not find a convincing temporal association between SDB events and fetal distress, particularly within the HDP group. It is likely that most fetuses (particularly those without severe uteroplacental insufficiency/FGR) are well protected from the transient maternal hypoxaemia of mild SDB, due to a combination of fetal adaptive behaviours and the high affinity fetal haemoglobin-oxygen dissociation curve. ${ }^{242}$ For example, when the hypoxia is acute, a fetus can increase its heart rate and cardiac output to increase $\mathrm{O}_{2}$ uptake from its placenta. It may preferentially redirect blood and hence oxygen from its periphery to its vital organs such as the brain, heart and adrenals. ${ }^{405,406}$ When the fetus is chronically hypoxic it is also capable of increasing its haemoglobin levels to increase its $\mathrm{O}_{2}$ carrying capacity to higher than that in adult haemoglobin. ${ }^{405}$

Studies in fetal sheep have confirmed that the fetus can sustain its protective circulatory adjustments and maintain fetal $\mathrm{O}_{2}$ consumption during even prolonged hypoxaemia, but as progressive metabolic acidosis develops, these protective adaptations begin to fail. ${ }^{414-416}$ This is consistent with our observations that fetuses with FGR may have exhausted their adaptive capacity and be at greater risk of fetal heart rate decelerations overnight, compared to well-grown fetuses which have better reserves to cope.

Further, any effect of maternal hypoxaemia may have been attenuated by the relatively mild degree of SDB. The two early case studies ${ }^{241,413}$ demonstrating fetal heart rate changes presented women with very severe SDB. Representative of the general population, our cohort mostly had mild SDB, yet those with the most severe disease (RDI's of 108.8 and 83.6) and a valid CTG did demonstrate fetal events. Overall, it seems likely that two pathways may drive fetal response to sleep. Both the severity of underlying placental insufficiency/FGR, and the severity of SDB/maternal physiological disturbance may determine whether fetal compromise occurs in association with respiratory events during sleep. 
Our results support the latest and largest studies in this area. Olivarez et al. ${ }^{107}$ performed at least 3 hours of fetal heart rate monitoring with concurrent PSG in 100 women. Among the 20 women diagnosed with mild to moderate SDB, the only fetal heart rate events that occurred were variable decelerations that were of appropriate gestational age, and none of the apnoeic events were associated with any fetal tracing abnormality. In a sample of 41 pregnant women, Fung et al. ${ }^{242}$ recorded an average of 7 hours of CTG, with $80 \%$ of respiratory events captured accompanied by successful fetal heart rate monitoring. Only one SDB patient out of 14 showed acute fetal heart rate responses and these occurred in a fetus who was severely growth restricted at birth. This study supports our data that even mild SDB events may be poorly tolerated in growth restricted fetuses who may have exhausted their oxygen reserves and adaptive capacities and are more likely to decompensate. This is the focus of our ongoing research.

\subsubsection{Chronic Impact of Sleep-Disordered Breathing on Fetal Wellbeing}

\subsubsection{Fetal Growth}

Measurement of fetal size by ultrasonography reflects fetal growth, health and wellbeing. Fetal weight estimates commonly play a pivotal role in management decisions, given that birthweight correlates with neonatal survival. ${ }^{222}$ Growth restricted infants have higher risks of neonatal complications such as birth hypoxia, ${ }^{230}$ necrotizing enterocolitis, respiratory distress and neonatal death, ${ }^{417}$ and growing evidence suggests that there are long-term metabolic consequences of FGR, such as increased risks of insulin resistance and cardiovascular complications. ${ }^{231}$ The degree of FGR has also been strongly associated with adverse outcome, stillbirth and neonatal death. ${ }^{418,419}$

Growth failure is typically referred to as FGR, and is described as a failure to achieve predetermined genetic growth potential due to the restrictions of an adverse intrauterine environment. ${ }^{228}$ Traditionally, FGR is defined as an EFW of below the $10^{\text {th }}$ percentile for gestational age on a standardised population growth curve whilst still in utero, with the term small for gestational age used for the neonate, defined as a birthweight of below the $10^{\text {th }}$ percentile. However, these terms are often used interchangeably.

We evaluated third trimester fetal growth trajectory as well as final birthweight, because an important indicator of placental insufficiency is fetal growth trajectory in utero, even among infants confirmed to be appropriate for gestation age (between $10^{\text {th }}$ and $90^{\text {th }}$ percentile) at birth. These fetuses with reduced growth velocity have been shown to display antenatal, intrapartum and postnatal indicators of placental insufficiency and so can also be considered 'growth restricted'. ${ }^{420}$

\subsection{Fetal Growth and Hypertensive Disease}

One of the leading maternal causes of FGR is hypertension in pregnancy. ${ }^{233}$ Reduced uteroplacental perfusion associated with maternal vascular disease is responsible for $25-30 \%$ of FGR cases; it is the most common cause in non-anomalous fetuses 200 The way in which PE affects the placenta was described in Chapter 4, but in short, errors in vascular remodelling with deficient trophoblast invasion 
during placentation create an area with high resistance to blood flow and resultant decreased perfusion of the intervillous space. This reduced placental perfusion is followed by placental ischemia/hypoxia which leads to widespread maternal endothelial dysfunction. ${ }^{324,325}$ Higher BP during pregnancy has been associated with lower fetal weight and neonatal birthweight, including within normotensive women, ${ }^{421,422}$ and women with PE have increased risks of having infants of low birthweight or small for gestational age. ${ }^{422-424}$ Studies focusing on GH and fetal growth have been inconsistent, from suggesting that women with $\mathrm{GH}$ are more likely to have small for gestational age infants, ${ }^{423,424}$ to finding that the rate of growth complications in $\mathrm{GH}$ were similar to that observed in normotensive pregnancies. ${ }^{145-147,425}$

Analysis of fetal growth across our sample of hypertensive and normotensive women confirmed that PE was associated with numerous measures of impaired fetal growth, including a greater fall in growth in the third trimester, lower birthweight and birthweight customised centile, and a higher rate of FGR at birth. Conversely however, the GH group did not differ from the normotensive women in any measures of fetal growth. This is consistent with other studies that have compared fetal growth in pregnancies complicated by hypertensive disease, and consistently found that PE significantly increased the risk of low birthweight and small for gestational age infants (aORs of $1.3-2.8) .{ }^{145-147,425}$

\subsection{Fetal Growth and Sleep-Disordered Breathing in Hypertensive and Normotensive Pregnancies}

The potential for hypoxic events at night to impact on fetal growth is evidenced by the known association with other disorders of maternal gas exchange, such as living at high altitude or cardio-respiratory disease. $202,426,427$ Chronic hypoxia slows fetal growth and reduces the pregnancy-associated rise in uterine artery blood flow. ${ }^{202,428}$ Recent studies have reported a 3-4 fold increase in small for gestational age infants among women residing at high compared to low altitude. ${ }^{429,430}$ Finer scale analyses revealing that birthweight reduction begins gradually and becomes marked at altitudes $>2000 \mathrm{~m}$ (the altitude where arterial oxygen saturation measurably begins to fall). ${ }^{431}$ Experimentally induced reduction of uterine blood flow in animal models and lower maternal umbilical artery blood flows observed clinically are clearly associated with reductions in placental weight that can be detected well before any attenuation in fetal growth. ${ }^{432,433}$ In a study of 14 cases of human FGR, the degree of fetal hypoxia correlated with placental weight. 434 In terms of cardio-respiratory disease, the average birthweight and birthweight percentile of neonates born to mothers with heart disease is significantly lower than the average noted in normal uncomplicated pregnancies. ${ }^{435,436}$

SDB is a unique model of maternal hypoxia because, although it is intermittent, it is associated with acute changes in sympathetic activity, which may further impact placental perfusion. A key compensatory mechanism for the fetus facing hypoxia is a redistribution of cardiac output and available oxygen, often resulting in FGR. There is an increase in the fetus's BP and it preferentially redirects peripheral blood, oxygen and nutrients to its vital organs such as the brain, heart and adrenals, but at the expense of the rest of its body. ${ }^{229,437}$ Fetal growth slows, altered fetal body proportions become 
evident on ultrasonography, the fetus may fall under the $10^{\text {th }}$ centile and the pattern of redistribution of the fetal blood flow can be detected by Doppler analysis. ${ }^{229}$

Several studies have demonstrated a link between SDB and impaired fetal growth. Initially, the largest studies involved self-reported snoring only and many concluded that chronic or severe snoring was associated with low birthweight and FGR at birth,6,279,281 Although questionnaire-based measurements of SDB have limitations, ${ }^{106-109}$ women diagnosed with SDB prior to pregnancy have also been confirmed to have an increased risk of having low birthweight infants $(<2500 \mathrm{~g})$ and small for gestational age infants. ${ }^{280}$ In a recent study by Fung et al., ${ }^{242}$ impaired fetal growth (defined as either FGR or a fall in customised fetal weight centile of $>33 \%$ across the third trimester) was observed in almost half of women with PSG-confirmed SDB versus only $10 \%$ of controls. Similarly, Pamidi et al. ${ }^{438}$ found that the odds of delivering a small for gestational age infant were significantly increased with a PSG-based diagnosis of maternal SDB using an AHI cut-off of 10.

Conversely, a number of studies contradict these findings. Within normotensive samples, no differences in infant birthweight and rate of growth restriction were reported between frequent snorers and nonsnorers in pregnancy in several studies. ${ }^{102,255,282}$ Some of the largest retrospective and longitudinal studies utilising PSG during pregnancy also found no association between SDB variables, low birthweight and small for gestational age infants. 7,124,262,263

In part, these inconsistencies may be due to inadequate adjustment for confounding variables, such as maternal obesity (a significant risk factor for FGR) and hypertension. No studies to date have addressed how SDB may affect fetal growth within hypertensive pregnancies, with attention to the confounding effect of BMI. In our study it was interesting to note that the neonates of women with hypertension and SDB had a higher birthweight and birthweight centile, and fewer occurrences of FGR at birth, compared to hypertensive women without comorbid SDB. Furthermore, these findings do not intuitively appear to be related to mild SDB across the cohort; those with moderate to severe SDB also had a lower incidence of FGR at birth, with infants that were around 20 customised centiles larger than those of mothers with an $\mathrm{RDI}<15$. This replicates previous findings of a positive association between infant birthweight and symptoms of SDB. ${ }^{253,254,279}$ Interestingly, it also ties in with our maternal results in Chapter 4 whereby those with HDP and moderate to severe SDB have a number of better outcomes, such as prolongation of gestation post diagnosis of HDP and overall, and lower levels of PE biomarkers of disease severity.

We did not find any evidence for slowed third trimester growth trajectory within the theoretically compromised group of women with SDB and HDP. This may relate to the relatively mild degree of SDB but may also be a reflection of small numbers. A limitation of monitoring third trimester growth in HDP, particularly PE, is that the maternal and fetal condition often mandates very pre-term delivery. Hence, the third trimester growth trajectory analysis only included fetuses well enough to complete a third trimester ultrasound and remain in utero for at least another two weeks before delivery, and subsequently a number of FGR fetuses, particularly within the No SDB group, were left out. 
Within the normotensive control women, SDB had no pathologic effects on fetal growth, with birthweight, birthweight customised centile, FGR at birth and slowed third trimester growth unrelated to SDB status.

As mentioned previously, a likely explanation for our study findings relates to the relatively mild degree of SDB and consequent lack of hypoxaemia experienced by the mother and hence the fetus. The average level of nocturnal hypoxaemia experienced was quite minimal, with a median of only three episodes of oxygen desaturations of $\geq 4 \%$ per hour. This would translate to only $1-2 \%$ of the night spent in a hypoxaemic state. In comparison, most studies referring to maternal oxygenation and fetal growth investigate much more severe degrees of hypoxia in animal models. For example, chronic hypoxia in rats results in significant growth restriction with associated fetal hypoxia and acidosis..$^{203,439}$ The degree of hypoxia imparted on the rats in these studies was substantial, with oxygen administered at levels reduced to a fractional oxygen concentration of $10 \%$ - equivalent to around $50 \% \mathrm{O}_{2}$ saturation. These animal models necessarily induce a very severe phenotype and are useful for exploring mechanisms. In contrast, SDB in human pregnancy has much more subtle physiological disturbances, and the interaction between underlying placental health as well as maternal contributors is likely to be key in identifying associations with clinically relevant outcomes.

Likewise, most information about maternal hypoxaemia and fetal growth in humans has been gathered from studies at high altitude, i.e. a state of continuous, rather than intermittent, maternal hypoxaemia. At sea level, the atmosphere contains $20.9 \%$ oxygen (fractional oxygen concentration $20.9 \%$ ). At an altitude of $1000 \mathrm{~m}$, the partial pressure of oxygen in air is equivalent to a fractional oxygen concentration of $18 \%$ at sea level, and at $2000 \mathrm{~m}$ to $16 \%$. In a study comparing blood gases in pregnancy, $\mathrm{PO}_{2}$ and oxygen saturation were lower on average in pregnancies at high altitude $(4300 \mathrm{~m})$ compared with sea level $(53 \mathrm{mmHg}$ and $89 \%$ vs $98.5 \mathrm{mmHg}$ and $98 \%$ respectively), whereas haemoglobin and arterial oxygen content were higher. 440 However, at high altitude oxygen saturation and haemoglobin decreased towards term resulting in a fall in arterial oxygen content at the end of pregnancy. These studies show that the decline in birthweight associated with altitude is actually curvilinear with the 'breakpoint' occurring around $2000 \mathrm{~m},{ }^{431}$ consistent with the shape of the haemoglobin-oxygen dissociation curve. ${ }^{441}$

If we looked at the women in our sample who had the most severe level of SDB (RDI $\geq 15$ ) we were able to identify a subgroup enduring oxygen desaturation an average of 30 times per hour. Despite this, moderate-to-severe SDB was actually positively associated with birthweight customised centile. Within the HDP group, fetal growth was favourable for those with even mild SDB. These may be chance observations alone, but this intriguing finding - together with the results in Chapter 4 - raises the possibility that we are observing the sequelae of slightly different PE phenotypes. PE not associated with SDB has its origins in primary placental ischaemia and abnormal placentation, a continuous and chronic hypoxic model, known to run an inexorably worsening course until delivery. It is possible that PE in the setting of SDB may run a longer and more benign course, the placenta partly protected by an intermittently hypoxic model with mostly normal oxygenation. While PE severity was similar at the time 
of recruitment between SDB and No SDB groups, the SDB group overall appeared to have a more attenuated progression. Experimental evidence suggests that fetal oxidative metabolism can be sustained despite reductions in fetal $\mathrm{O}_{2}$ delivery of $40-50 \%$, as long as fetal oxygen reserves are not depleted. ${ }^{412}$ The transient and reversible hypoxia of SDB-related PE may confer some maternal and fetal protection, but this interpretation is speculative. It warrants further investigation through a combination of well-designed animal models, and appropriately powered human studies, with robust laboratory and clinical outputs and careful adjusting for relevant confounding variables.

\subsubsection{Perinatal Outcomes}

Our study is the first to specifically look at how maternal SDB may compound the already negative effects of hypertensive disorders during pregnancy on fetal health. Our results suggest that concurrent SDB with HDP has no impact on delivery or the health of the infant at birth, above that attributed to hypertension alone. While we were underpowered for many of these individual adverse perinatal outcomes, there were also no apparent differences even when taken collectively. In fact, women with SDB were less likely to have a pre-term birth, and although not significant, those with moderate to severe levels of SDB had better outcomes including half the likelihood of requiring an emergency caesarean delivery, and no infants with 5 min Apgar scores $\leq 7$ or needing admission to NICU.

Within the normotensive group, we found no significant impact of SDB on perinatal outcomes with all mothers giving birth at term and no admissions to NICU. Our results support past studies comparing snorers to non-snorers, finding no differences in the occurrence of pre-term birth and infant admission to NICU, 279 and no differences in Apgar scores as an indication of fetal wellbeing at birth. $102,253,254,282$ Two studies using more comprehensive diagnosis of maternal SDB have performed PSG in the first and third trimester of pregnancy and found no associations between SDB variables and pre-term delivery. 7,263

In contrast, a few studies have found that an Apgar score of $\leq 7$ was more common in infants born to habitual snorers compared to non-snorers. ${ }^{6,279}$ Retrospective analysis of pregnant women with confirmed SDB found that they were more likely to have a caesarean delivery, preterm delivery, reduced Apgar scores and NICU admission compared to women without OSA, ${ }^{124,280}$ and compared to both controls who were obese and of normal weight. ${ }^{262}$ It should be noted that these studies generally had women exhibiting a more severe degree of SDB compared to our study.

As illustrated, the number of studies looking at various birth outcomes related to maternal SDB have inconsistent results, likely due to varying definitions and methodologies. Studies based on self-reported snoring and apnoea symptoms can sample a much larger group of women, but we now know that these measures are poor surrogates for PSG-confirmed SDB. ${ }^{106-109}$ Due to the difficulty in performing goldstandard PSG, studies employing these methods (including this study) are often limited by sample size and may be underpowered. This is clearly an area that requires further research to determine the true impact that maternal SDB has on fetal wellbeing. 


\subsubsection{Fetal Growth Hormones in Cord Blood}

To strengthen our results, we sought biochemical confirmation of growth impairment by measuring IGF1, IGF-2 and their respective binding proteins in cord blood at delivery. The IGF axis is an important regulator of growth, responsible for placental development and differentiation, nutrient transfer and fetal growth. ${ }^{208}$ Their respective binding proteins serve many functions, for example they prolong the half-life of IGF and provide a circulating storage reservoir for IGF.

Numerous studies have demonstrated the importance of IGFs actions for fetal growth and development. Measurement of IGF levels in cord blood from healthy newborns demonstrated that birthweight is positively correlated with IGF-1209,210 whereas levels are low in small for gestational age infants. ${ }^{211}$ There are numerous reports of increased maternal IGFBP-1 levels in pregnancy complications such as FGR. ${ }^{209,212}$ Reduced placental size followed by FGR in mice lacking the IGF-2 gene suggests that IGFs influence fetal growth through a role in promoting normal placental development, ${ }^{442}$ whereas IGFBP-2 levels in cord blood are increased among human fetuses with FGR. ${ }^{213}$

\subsection{Fetal Growth Hormones in Cord Blood and Sleep-Disordered Breathing}

Numerous studies have investigated fetal hypoxia and the IGF axis. Animal models have demonstrated that decreased fetal oxygen availability decreases IGF-1 production and availability with increased concentrations of IGFBP-1.443 FGR caused by maternal hypoxia in rats has been related to increased IGFBP-1 and IGFBP-2 expression. ${ }^{444}$ In human fetuses experiencing profound and prolonged hypoxia, IGFBP-1 levels were markedly elevated compared to acutely hypoxic and normal babies. ${ }^{445}$

We are aware of only one study that has investigated the IGF axis in pregnancies affected by SDB, ${ }^{242}$ which found that IGF-1 in cord blood was lower among infants of women with SDB compared to controls, with a corresponding increase in IGFBP-1 and IGFBP-2. Whereas these findings by Fung et al.242 paralleled the clinical growth parameters in the expected directions, our results showed the opposite with normotensive participants with SDB demonstrating increased levels of IGF-1 and IGF-2, and decreased levels of IGFBP-2. Within the HDP group, there was no impact of SDB on any IGF values. These results are not surprising given that fetal growth was not impaired by the presence of maternal SDB.

Few studies have looked at other biological signs of impaired fetal oxygenation in maternal SDB. Ravishankar and colleagues ${ }^{234}$ assessed the placentas of women with SDB and habitual snoring for markers of chronic hypoxia and uteroplacental underperfusion, and found that compared to non-snoring controls, a marker of tissue hypoxia was more common in SDB placentas, however the uteroplacental underperfusion scores were similar. This perhaps lends some support to our contention that placental underperfusion and dysfunction may be less in women with SDB associated HDP than in those with primary placental disease. Women with SDB have been shown to have lower levels of estriol (uE3), a marker of fetoplacental unit wellbeing, compared to controls. ${ }^{221}$ Lastly, self-reported habitual snoring 
has been associated with increased fetal erythropoiesis (production of red blood cells) manifested by elevations of circulating nRBC's, EPO and IL-6 in cord blood (typically seen with chronic fetal hypoxia) compared with non-snorers, despite no differences in birthweight centile. ${ }^{206}$ Again, the use of selfreported snoring to define SDB limits the interpretation of these findings.

\subsubsection{Strengths and Limitations}

Key strengths of our study relate to the comprehensive assessments employed to determine the role SDB may play on fetal oxygenation and development. In particular, we used CTG to collect real-time measures of fetal oxygenation in response to maternal apnoea and hypoxaemia. As mentioned a few previous studies have used this technique, however our study had greater success at gathering multiple hours of continuous monitoring on a large number of women. As described, we captured $>75 \%$ of total sleep time with successful fetal heart rate on two-thirds of our participants, and those included in the analysis had an average success rate of $>90 \%$ with approximately six hours of concurrent PSG and CTG data recorded on average.

In comparison, Olivarez et al. ${ }^{107}$ reported that their participants were monitored for 3-6 hours, however their data states that within the 20 participants with SDB the mean AHI was 12.2/hr and an average of 12.2 respiratory events were captured during the monitoring period. This would imply that on average, only one hour of sleep study data per participant was recorded with concurrent CTG. In an attempt to evaluate the success rate of adding continuous CTG during full night PSG, Reid et al. ${ }^{446}$ considered that $>50 \%$ of interpretable fetal heart rate tracing was ideal, but $25-50 \%$ was acceptable. In our experience, $<50 \%$ of interpretable trace made it very difficult to confidently assess fetal condition in response to sleep events, particularly as the available data was typically intermittent rather than in large continuous periods. In Reid et al.'s ${ }^{446}$ study, $>50 \%$ interpretable trace was obtained in just over half of their sample, compared to $82 \%$ of our sample. They also reported a much lower success rate with obese women and mothers with SDB, whereas monitoring success was unrelated to BMI and SDB in our sample.

The technique used for investigating whether maternal SDB affects fetal growth, which was based on our previous study ${ }^{242}$ and included the collection of cord blood to analyse hormonal markers of fetal growth, was more comprehensive than others in the field. Previous studies using birthweight or small for gestational age based on population standards as the sole measure of placental insufficiency may be inadequate..$^{7,262,263,280}$ The categorisation of $F G R$ as $<10^{\text {th }}$ percentile has often been criticised secondary to the inclusion of many fetuses that are constitutionally small and not at risk for poor perinatal outcome. ${ }^{447,448}$ Also, assuming adequate uteroplacental functioning when birthweight is above $10^{\text {th }}$ centile has limitations in identifying the fetus that is failing to achieve its growth potential. ${ }^{449,450}$ For example, it is possible to have a fetus that is above the $80^{\text {th }}$ percentile on a population growth curve during pregnancy but is born at the $20^{\text {th }}$ percentile - technically not small for gestational age. However, this fetus has not achieved its genetically predetermined growth potential and may still be at risk for poor perinatal outcome. ${ }^{451}$ 
To overcome these limitations, we customised both the ultrasound estimate of fetal weight and birthweight according to maternal characteristics known to affect birthweight. This is particularly important in large women (a high proportion of our sample) where significant growth restriction may be present, yet the fetus is not below the $10^{\text {th }}$ percentile at delivery based on population standards. As an example, within our sample a $2840 \mathrm{~g}$ infant delivered at $38+0$ weeks to a mother with a pre-pregnancy BMI of $38 \mathrm{~kg} / \mathrm{m}^{2}$ was considered as $<10 \%$ based on customised centiles, ${ }^{299}$ however on a population standard calculator ${ }^{452}$ the infant was measured at the $17^{\text {th }}$ percentile. Secondly, we evaluated third trimester fetal growth trajectory. In studies which have serially evaluated fetal growth, slowing of fetal growth trajectory has been associated with a higher risk of delivering a small for gestational age infant, with a corresponding increase in neonatal morbidity. ${ }^{453}$ In pregnancies with appropriate for gestational age sized fetuses, slowing third trimester growth has been associated with adaptive cerebral blood flow patterns associated with in utero hypoxia, increased rates of fetal compromise during labour, 454 neonatal acidosis and reduced neonatal body fat percentage. ${ }^{420}$

We acknowledge that this study also had its shortcomings. As discussed in Chapter 4, the most notable limitation of our study related to sample size. Although adequate for our primary analysis comparing the prevalence of SDB in hypertensive versus normotensive pregnancies (Chapter 3), after comparing those with SDB to those without SDB and splitting the sample into HDP and control groups, the numbers in each analysis were reduced. Positive findings thus need to be treated with caution and tested in future adequately powered studies. PSG is considered the gold standard method of diagnosing SDB, however this methodology is intensive. Along with the continuous CTG, need for ultrasound and collection of maternal and cord blood samples, our study required significant commitment from the participants and hence recruitment for this study was challenging.

The amount of data available for each outcome was limited by a number of factors, mostly beyond our control. Firstly, we had a high rate of successful CTG tracing in comparison to previous studies, however we still had to exclude 23 participants for substandard CTGs and unfortunately this included half of the PE participants. Secondly, fetal growth within the PE group was difficult to assess and interpret given the number of growth restricted fetuses ultimately requiring early intervention and delivery. Assessment of fetal growth trajectory was limited because these fetuses were already below the $10^{\text {th }}$ centile in the third trimester. We also need to keep in mind that ultrasound estimates of fetal weight are subject to error, with an average error of around $8 \% .222$ Regardless of these issues, our results were clearly not in the hypothesised directions and unlikely to be altered by a larger sample size. Lastly, a number of cord blood samples were unable to be collected, mostly due to logistical issues.

Another limitation in our study and others, is the predominance of mild SDB in pregnant cohorts, ,7263 as previously discussed. Theoretically we proposed that fetal oxygenation and uteroplacental perfusion would be primarily affected by maternal hypoxaemia as a result of repeated apnoea. The number and severity of oxygen desaturations on average was quite low, attenuating any potential impact on the fetus. However, there were 12 mothers with untreated moderate to severe SDB with frequent oxygen 
desaturations in this study - none of the fetuses of these women showed significant signs of nocturnal distress on CTG, and only one infant was delivered preterm with FGR.

\subsubsection{Conclusion}

This study is an important addition to the current literature regarding the impact of maternal SDB on fetal wellbeing both in utero and at birth. Our data adds to the few studies so far using objective measures of SDB in this field, and our in-depth analysis of nocturnal fetal oxygenation and customised fetal growth opposes the hypothesis that SDB has a detrimental effect on fetal health. We found no evidence to support a relationship between SDB and CTG abnormalities, growth trajectory in the third trimester and fetal growth restriction at birth, perinatal outcomes or hormonal regulators of fetal growth. Interestingly, women with HDP and concurrent SDB and those with moderate to severe SDB had better outcomes in terms of customised infant size at birth and cord blood fetal growth hormones, compared to those without SDB. These findings are intriguing. Whether HDP that has origins (or contributions) from SDB may have a more benign course due to the intermittent nature of placental hypoxic insults is speculative, but warrants further study.

The fetus has considerable adaptive capacity to withstand in utero hypoxia, which may explain our mostly negative findings. In our study, FGR was uncommon and mostly mild, at the time of PSG. Likewise, SDB was mostly mild. Larger studies with more comprehensive measurement of the potential pathophysiological effects on the fetus associated with SDB are required to either confirm or refute our findings. It seems likely that fetal sequelae will only be unmasked in the setting of either more severe degrees of SDB and/or severe underlying FGR and placental disease. This is the focus of our ongoing work. 


\subsection{Supplementary Tables}

Table 5.9 Demographics for Preeclampsia and Gestational Hypertension Cases Stratified by SDB Status

\begin{tabular}{|c|c|c|c|c|c|c|}
\hline & \multicolumn{2}{|c|}{$\begin{array}{c}\text { PE } \\
(n=17)\end{array}$} & \multicolumn{4}{|c|}{$\begin{array}{c}\mathrm{GH} \\
(n=23)\end{array}$} \\
\hline & $\begin{array}{l}\text { SDB } \\
(n=7)\end{array}$ & $\begin{array}{l}\text { No SDB } \\
(n=10)\end{array}$ & $p$ & $\begin{array}{c}\text { SDB } \\
(n=14)\end{array}$ & $\begin{array}{c}\text { No SDB } \\
(n=9)\end{array}$ & $p$ \\
\hline Age (years) & $30.6 \pm 4.7$ & $32.0 \pm 4.9$ & .56 & $34.6 \pm 4.3$ & $32.0 \pm 3.8$ & .15 \\
\hline Nulliparous & 6 (85.7\%) & 7 (70.0\%) & .60 & 8 (57.1\%) & 5 (55.6\%) & 1.0 \\
\hline GDM & $3(42.9 \%)$ & $3(30.0 \%)$ & .58 & $2(14.3 \%)$ & $0(0.0 \%)$ & .50 \\
\hline BMI first appt & $33.1 \pm 9.6$ & $29.7 \pm 8.3$ & .45 & $34.2 \pm 5.7$ & $31.8 \pm 6.5$ & .36 \\
\hline Weight gain $(\mathrm{kg})$ & $9.9 \pm 5.8$ & $7.3 \pm 5.8$ & .38 & $12.3 \pm 4.7$ & $10.1 \pm 7.0$ & .39 \\
\hline $\mathrm{BMI}$ at PSG & $37.3 \pm 9.1$ & $32.3 \pm 7.8$ & .24 & $38.5 \pm 4.8$ & $35.4 \pm 6.1$ & .19 \\
\hline $\begin{array}{l}\text { Gestation at } \\
\text { PSG (weeks) }\end{array}$ & $30.6 \pm 3.5$ & $31.8 \pm 3.0$ & .45 & $35.7 \pm 1.5$ & $34.5 \pm 3.8$ & .39 \\
\hline $\mathrm{RDI} / \mathrm{hr}$ & $\begin{array}{c}16.8(12.3 \\
108.6)\end{array}$ & $1.9(1.5,3.0)$ & $<.001$ & $\begin{array}{c}13.4(7.1 \\
28.5)\end{array}$ & $3.0(1.1,3.2)$ & $<.001$ \\
\hline ODI $\geq 3 \%$ overall & $\begin{array}{c}6.0(0.4 \\
109.7)\end{array}$ & $0.7(0.2,1.4)$ & .055 & $8.7(3.0,36.3)$ & $1.3(0.0,2.9)$ & .002 \\
\hline ODI $\geq 4 \%$ overall & $\begin{array}{c}1.9(0.0 \\
101.0)\end{array}$ & $0.1(0.0,0.7)$ & .055 & $4.0(0.4,23.4)$ & $0.3(0.0,1.3)$ & .01 \\
\hline
\end{tabular}

Note. Values given as $\mathrm{M} \pm \mathrm{SD}$, Mdn (IQR), or $\mathrm{n}(\%)$. SDB = sleep-disordered breathing, $\mathrm{PE}=$ preeclampsia, $\mathrm{GH}=$ gestational hypertension, $\mathrm{GDM}=$ gestational diabetes mellitus, $\mathrm{BMI}=$ body mass index $\mathrm{kg} / \mathrm{m}^{2}, \mathrm{PSG}=$ polysomnography, $\mathrm{RDI}=$ respiratory disturbance index, $\mathrm{ODI}=$ oxygen desaturation index. 
Table 5.10 Cardiotocography Variables across Hypertensive Groups

\begin{tabular}{lcccc}
\hline & $\begin{array}{c}\text { PE } \\
(\mathrm{n}=7)\end{array}$ & $\begin{array}{c}\mathrm{GH} \\
(\mathrm{n}=20)\end{array}$ & $\begin{array}{c}\text { Control } \\
(\mathrm{n}=32)\end{array}$ & $\mathrm{p}$ \\
\hline \% CTG success & $90.6 \pm 12.9$ & $95.5 \pm 7.2$ & $91.9 \pm 10.7$ & .37 \\
Hours of CTG & $5.5 \pm 1.4$ & $6.1 \pm 1.3$ & $6.0 \pm 1.2$ & .54 \\
Gestation CTG* & $31.6 \pm 3.7 \mathrm{a}$ & $36.1 \pm 1.4 \mathrm{~b}$ & $33.5 \pm 2.0_{\mathrm{c}}$ & $<.001$ \\
BMI CTG & $34.1 \pm 7.6$ & $37.6 \pm 5.5$ & $36.2 \pm 6.4$ & .43 \\
RDI/hr & $6.9(4.5,16.8)$ & $6.9(3.2,20.8)$ & $4.1(2.3,7.7)$ & .27 \\
\hline Any event & $4(57.1 \%)$ & $6(30.0 \%)$ & $10(31.3 \%)$ & .38 \\
$\mathrm{n}(\%)$ & $3.8 \pm 1.0 \mathrm{a}$ & $2.0 \pm 1.3 \mathrm{~b}$ & $2.0 \pm 0.8 \mathrm{~b}$ & .02 \\
Total events/night* & $2(28.6 \%)$ & $0(0.0 \%)$ & $1(3.1 \%)$ & .009 \\
Event 1 n (\%) & $4(57.1 \%)$ & $6(30.0 \%)$ & $10(31.3 \%)$ & .38 \\
Event 4 n (\%) & & & & \\
\hline
\end{tabular}

Note. Values given as $\mathrm{M} \pm \mathrm{SD}$, Mdn (IQR) or $\mathrm{n}(\%) . \mathrm{PE}=$ preeclampsia, $\mathrm{GH}$ = gestational hypertension, $\mathrm{CTG}=$ cardiotocography, $\mathrm{BMI}=$ body mass index $\mathrm{kg} / \mathrm{m}^{2}, \mathrm{RDI}=$ respiratory disturbance index. Means in the same row that do not share subscript differ at $p<.05$ in the Newman-Keuls significant difference comparison. Event 2 and 3 omitted from this table due to no events.

*only those who had fetal heart rate events.

Event $1=$ Prolonged bradycardia ( $>15 \mathrm{bpm}$ fall below baseline for $\geq 90 \mathrm{sec}$ and $<5 \mathrm{~min}$ ), Event $4=$ deceleration lasting between $60-90 \mathrm{sec}$ with $>15 \mathrm{bpm}$ fall from baseline. 
Table 5.11 Key Fetal Outcome Measures for those with and without Maternal SDB at an RDI $\geq 15$

\begin{tabular}{|c|c|c|c|}
\hline & ${ }^{*} \mathrm{SDB}$ & No SDB & $p$ \\
\hline $\mathrm{RDI} / \mathrm{hr}$ & $32.9(23.2-52.6)$ & $3.7(1.9-6.6)$ & $<.001$ \\
\hline ODI $\geq 3 \%$ & $32.9(21.9-42.2)$ & $1.3(0.3-3.2)$ & $<.001$ \\
\hline Birth gestation (weeks) & $38.0 \pm 1.3$ & $37.5 \pm 3.7$ & .43 \\
\hline Birthweight (g) & $3313.8 \pm 625.5$ & $2995.5 \pm 1041.7$ & .31 \\
\hline Birth cust. centile (\%) & $52.6 \pm 30.9$ & $34.0 \pm 31.8$ & .06 \\
\hline FGR at recruitment & $1(8.3 \%)$ & $9(13.4 \%)$ & 1.0 \\
\hline FGR at birth ( $<10^{\text {th }}$ cust. centile $)$ & $1(8.3 \%)$ & $24(35.8 \%)$ & .09 \\
\hline Impaired fetal growth (FGR or fall in & $3(25.0 \%)$ & $33(49.3 \%)$ & 21 \\
\hline Participants with valid T3 scan & $n=11$ & $n=52$ & \\
\hline T3 cust. centile (\%) & $57.7 \pm 31.7$ & $53.3 \pm 27.9$ & .64 \\
\hline Birthweight cust. centile (\%) & $48.8 \pm 29.3$ & $41.8 \pm 31.2$ & .50 \\
\hline Change in cust. centile, total (\%) & $-8.9 \pm 24.3$ & $-11.6 \pm 24.1$ & .73 \\
\hline Change in cust. centile per day (\%) & $-0.47 \pm 0.96$ & $-0.40 \pm 0.88$ & .81 \\
\hline Fall in cust. centile $>33 \%$ over 6 weeks & $3(27.3 \%)$ & $11(21.2 \%)$ & .70 \\
\hline Perinatal Outcomes & $n=12$ & $n=67$ & \\
\hline Preterm Birth $<37$ weeks & $1(8.3 \%)$ & $17(25.4 \%)$ & .28 \\
\hline Caesarean \% & $8(66.7 \%)$ & $39(58.2 \%)$ & .75 \\
\hline Emergency caesarean & $2(16.7 \%)$ & $25(37.3 \%)$ & .20 \\
\hline Apgar $1 \min \leq 7$ & $2(16.7 \%)$ & $15(22.7 \%)$ & 1.0 \\
\hline Apgar $5 \min \leq 7$ & $0(0.0 \%)$ & $4(6.1 \%)$ & 1.0 \\
\hline NICU admit & $0(0.0 \%)$ & $8(12.1 \%)$ & .35 \\
\hline SCN admit & $2(16.7 \%)$ & $13(19.7 \%)$ & 1.0 \\
\hline Cord Blood & $n=11$ & $n=44$ & \\
\hline IGF-1 (pg/ml) & $6564.5 \pm 3274.9$ & $5303.7 \pm 4137.3$ & .35 \\
\hline IGF-2 (pg/ml) & $108175.5 \pm 41213.7$ & $80394.1 \pm 44939.5$ & .07 \\
\hline IGFBP-1 (ng/ml) & $12.5 \pm 6.7$ & $19.7 \pm 12.3$ & .07 \\
\hline IGFBP-2 (ng/ml) & $159.1 \pm 102.1$ & $167.3 \pm 101.3$ & .81 \\
\hline Gestation Sample Taken & $38.1 \pm 1.3$ & $37.3 \pm 3.7$ & 27 \\
\hline
\end{tabular}

Note. Values given as $\mathrm{M} \pm \mathrm{SD}$, Mdn (IQR), or $\mathrm{n}(\%)$. SDB = sleep-disordered breathing, $\mathrm{RDI}=$ respiratory disturbance index, $\mathrm{ODI}=$ oxygen desaturation index, cust. $=$ customised, $\mathrm{FGR}=$ fetal growth restriction, $\mathrm{T} 3=$ third trimester, $\mathrm{NICU}=$ neonatal intensive care unit, $\mathrm{SCN}=$ special care nursery, IGF $=$ insulin-like growth factor, IGFBP $=$ insulin-like growth factor binding protein.

*excludes 3 CPAP users. 
Table 5.12 Measures of Fetal Growth for those with and without SDB across Preeclampsia and Gestational Hypertension Groups

\begin{tabular}{|c|c|c|c|c|c|c|}
\hline & \multicolumn{2}{|c|}{ PE } & \multicolumn{4}{|c|}{$\mathrm{GH}$} \\
\hline & $\mathrm{SDB}^{*}$ & No SDB & $p$ & SDB & No SDB & $p$ \\
\hline All participants & $n=6$ & $n=10$ & & $n=14$ & $n=9$ & \\
\hline $\mathrm{RDI} / \mathrm{hr}$ & $15.2(11.0,56.9)$ & $1.9(1.5,3.0)$ & $<.001$ & $13.4(7.1,28.5)$ & $3.0(1.1,3.2)$ & $<.001$ \\
\hline Birth gestation (weeks) & $32.1 \pm 4.8$ & $33.0 \pm 3.5$ & .68 & $38.1 \pm 1.6$ & $37.0 \pm 2.9$ & .26 \\
\hline Birthweight (g) & $1591.3 \pm 1123.7$ & $1648.5 \pm 725.1$ & .90 & $3434.5 \pm 693.8$ & $2863.2 \pm 918.2$ & .10 \\
\hline Birth cust. centile (\%) & $11.7 \pm 18.1$ & $3.3 \pm 3.6$ & .16 & $56.6 \pm 37.0$ & $30.5 \pm 34.4$ & .10 \\
\hline FGR at recruitment & $4(66.7 \%)$ & $6(60.0 \%)$ & 1.0 & $0(0.0 \%)$ & $0(0.0 \%)$ & 1.0 \\
\hline FGR at birth & $4(66.7 \%)$ & $10(100.0 \%)$ & .13 & $2(14.3 \%)$ & $3(33.3 \%)$ & .34 \\
\hline $\begin{array}{l}\text { Impaired fetal growth (FGR or } \\
\text { fall in cust. centile }>33 \% \text { ) }\end{array}$ & $5(83.3 \%)$ & $10(100.0 \%)$ & .38 & $6(42.9 \%)$ & $5(55.6 \%)$ & .68 \\
\hline Participants with T3 scan & $n=3$ & $n=3$ & & $n=12$ & $n=6$ & \\
\hline $\mathrm{RDI} / \mathrm{hr}$ & $8.4(7.5,18.9)$ & $2.9(1.3,4.0)$ & $<.001$ & $14.9(7.8,35.6)$ & $1.7(0.8,3.1)$ & $<.001$ \\
\hline T3 US gestation (weeks) & $33.3 \pm 0.7$ & $33.0 \pm 1.0$ & .67 & $34.1 \pm 1.5$ & $32.7 \pm 2.7$ & .17 \\
\hline T3 cust. centile (\%) & $56.1 \pm 30.5$ & $28.9 \pm 35.2$ & .37 & $65.1 \pm 32.8$ & $53.8 \pm 35.8$ & .51 \\
\hline Birth gestation (weeks) & $36.4 \pm 1.4$ & $35.8 \pm 1.3$ & .60 & $38.4 \pm 1.3$ & $37.0 \pm 3.2$ & .21 \\
\hline Birthweight (g) & $2558.7 \pm 590.7$ & $2171.0 \pm 625.3$ & .48 & $3496.5 \pm 595.9$ & $3040.7 \pm 988.5$ & .24 \\
\hline Birthweight cust. centile (\%) & $23.5 \pm 20.1$ & $5.5 \pm 4.8$ & .21 & $57.9 \pm 35.1$ & $40.9 \pm 38.0$ & .36 \\
\hline $\begin{array}{l}\text { Change in cust. centile total } \\
(\%)\end{array}$ & $-32.7 \pm 23.8$ & $-23.4 \pm 31.6$ & .71 & $-7.4 \pm 35.5$ & $-12.9 \pm 22.1$ & .74 \\
\hline $\begin{array}{l}\text { Change in cust. centile per } \\
\text { day }(\%)\end{array}$ & $-1.56 \pm 1.11$ & $-1.42 \pm 2.00$ & .93 & $-0.49 \pm 1.36$ & $-0.39 \pm 0.58$ & .87 \\
\hline Fall in cust. centile $>33 \%$ & 2 (66.6\%) & 1 (33.3\%) & 1.0 & 4 (33.3\%) & $2(33.3 \%)$ & 1.0 \\
\hline
\end{tabular}

Note. Values given as $\mathrm{M} \pm \mathrm{SD}$, Mdn (IQR), or $\mathrm{n}(\%)$. SDB = sleep-disordered breathing, $\mathrm{PE}=$ preeclampsia, $\mathrm{GH}=$ gestational hypertension, $\mathrm{RDI}=$ respiratory disturbance index, cust. $=$ customised, FGR $=$ fetal growth restriction $\left(<10^{\text {th }}\right.$ cust. centile $), \mathrm{T} 3=$ third trimester, US $=$ ultrasound. ${ }^{*}$ excludes $1 \mathrm{CPAP}$ user. 
Table 5.13 Measures of Fetal Growth across Hypertensive Groups

\begin{tabular}{|c|c|c|c|c|}
\hline & $P E^{\star}$ & $\mathrm{GH}$ & Control $^{*}$ & $p$ \\
\hline All participants & $n=16$ & $n=23$ & $n=40$ & \\
\hline $\mathrm{RDI} / \mathrm{hr}$ & $3.5(1.7,13.3)$ & $6.6(3.0,14.9)$ & $4.0(2.2,7.5)$ & .26 \\
\hline Birth gestation (weeks) & $32.7 \pm 3.9 a$ & $37.7 \pm 2.2 \mathrm{~b}$ & $39.5 \pm 1.2_{c}$ & $<.001$ \\
\hline Birthweight (g) & $1627.1 \pm 858.6 \mathrm{a}$ & $3211.0 \pm 820.0 b$ & $3514.4 \pm 493.1_{b}$ & $<.001$ \\
\hline $\begin{array}{l}\text { Birthweight cust. centile } \\
\text { (\%) }\end{array}$ & $6.4 \pm 11.6 \mathrm{a}$ & $46.4 \pm 37.5 b$ & $43.5 \pm 27.3 b$ & $<.001$ \\
\hline FGR at recruitment & 10 (62.5\%) & $0(0 \%)$ & $0(0 \%)$ & $<.001$ \\
\hline $\begin{array}{l}\text { FGR at birth }\left(<10^{\text {th }} \text { cust. }\right. \\
\text { centile })\end{array}$ & $14(87.5 \%)$ & $5(21.7 \%)$ & $6(15.0 \%)$ & $<.001$ \\
\hline $\begin{array}{l}\text { Impaired fetal growth } \\
\text { (FGR or fall in cust. } \\
\text { centile }>33 \% \text { ) }\end{array}$ & 15 (93.8\%) & $11(47.8 \%)$ & $10(25.0 \%)$ & $<.001$ \\
\hline Participants with T3 scan & $n=6$ & $n=18$ & $n=39$ & \\
\hline $\mathrm{RDI} / \mathrm{hr}$ & $2.5(1.8,28.3)$ & $6.9(3.0,16.9)$ & $4.0(2.2,7.5)$ & .26 \\
\hline T3 US gestation (weeks) & $33.2 \pm 0.8$ & $33.7 \pm 2.0$ & $33.0 \pm 1.6$ & .34 \\
\hline T3 cust. centile (\%) & $42.5 \pm 33.0$ & $61.3 \pm 33.2$ & $52.6 \pm 25.0$ & .32 \\
\hline Birth gestation (weeks) & $36.1 \pm 1.2 \mathrm{a}$ & $37.9 \pm 2.2 \mathrm{~b}$ & $39.5 \pm 1.2_{c}$ & $<.001$ \\
\hline Birthweight (g) & $2364.8 \pm 584.0 \mathrm{a}$ & $3344.6 \pm 752.3 b$ & $3505.8 \pm 496.5 b$ & $<.001$ \\
\hline $\begin{array}{l}\text { Birthweight cust. centile } \\
\text { (\%) }\end{array}$ & $14.5 \pm 16.4 a$ & $52.3 \pm 35.9 b$ & $43.2 \pm 27.6 b$ & .031 \\
\hline $\begin{array}{l}\text { Change in cust. centile, } \\
\text { total (\%) }\end{array}$ & $-28.1 \pm 25.5$ & $-9.2 \pm 31.1$ & $-9.5 \pm 19.1$ & .19 \\
\hline $\begin{array}{l}\text { Change in cust. centile } \\
\text { per day (\%) }\end{array}$ & $-1.49 \pm 1.45 a$ & $-0.46 \pm 1.14 b$ & $-0.23 \pm 0.44 b$ & .003 \\
\hline $\begin{array}{l}\text { Fall in cust. centile of } \\
>33 \% \text { over } 6 \text { weeks }\end{array}$ & $3(50.0 \%)$ & $6(33.3 \%)$ & $5(12.8 \%)$ & .051 \\
\hline
\end{tabular}

Note. Values given as $\mathrm{M} \pm \mathrm{SD}, \mathrm{Mdn}(\mathrm{IQR})$, or $\mathrm{n}(\%) . \mathrm{PE}=$ preeclampsia, $\mathrm{GH}$ = gestational hypertension, $\mathrm{RDI}=$ respiratory disturbance index, cust. $=$ customised, $\mathrm{FGR}=$ fetal growth restriction, $\mathrm{T} 3=$ third trimester, US = ultrasound. Means in the same row that do not share subscript differ at $p<.05$ in the Newman-Keuls significant difference comparison.

*excludes 3 CPAP users 
Table 5.14 Birth Outcomes across Hypertensive Groups

\begin{tabular}{lcccc}
\hline $\mathrm{n}(\%)$ & $\begin{array}{c}\mathrm{PE}^{*} \\
(\mathrm{n}=16)\end{array}$ & $\begin{array}{c}\mathrm{GH} \\
(\mathrm{n}=23)\end{array}$ & $\begin{array}{c}\text { Control }^{*} \\
(\mathrm{n}=40)\end{array}$ & $\mathrm{p}$ \\
\hline Preterm Birth $<37$ & $13(81.3 \%)$ & $5(21.7 \%)$ & $0(0.0 \%)$ & $<.001$ \\
Caesarean \% & $13(81.3 \%)$ & $17(73.9 \%)$ & $17(42.5 \%)$ & .007 \\
Emergency caesarean & $9(56.3 \%)$ & $10(43.5 \%)$ & $8(20.0 \%)$ & .02 \\
Apgar 1 min $\leq 7$ & $6(37.5 \%)$ & $6(26.1 \%)$ & $5(12.8 \%)$ & .11 \\
Apgar 5 min $\leq 7$ & $2(12.5 \%)$ & $1(4.3 \%)$ & $1(2.6 \%)$ & .31 \\
NICU admit & $7(43.8 \%)$ & $1(4.3 \%)$ & $0(0.0 \%)$ & $<.001$ \\
SCN admit & $5(31.3 \%)$ & $7(30.4 \%)$ & $3(7.7 \%)$ & .035
\end{tabular}

Note. $\mathrm{PE}=$ preeclampsia, $\mathrm{GH}=$ gestational hypertension, $\mathrm{NICU}=$ neonatal intensive care unit, $\mathrm{SCN}=$ special care nursery. ${ }^{*} 1 \mathrm{PE}$ and 2 controls excluded for CPAP use

Table 5.15 Birth Outcomes for those with and without SDB across Hypertensive Groups

\begin{tabular}{lcccccc}
\hline $\mathrm{n}(\%)$ & \multicolumn{2}{c}{$\begin{array}{c}\mathrm{PE} \\
(\mathrm{n}=16)\end{array}$} & \multicolumn{5}{c}{$\begin{array}{c}\mathrm{GH} \\
(\mathrm{n}=23)\end{array}$} \\
\cline { 2 - 7 } & $\begin{array}{c}\mathrm{SDB}^{*} \\
(\mathrm{n}=6)\end{array}$ & $\begin{array}{c}\text { No SDB } \\
(\mathrm{n}=10)\end{array}$ & $\mathrm{p}$ & $\begin{array}{c}\text { SDB } \\
(\mathrm{n}=14)\end{array}$ & $\begin{array}{c}\text { No SDB } \\
(\mathrm{n}=9)\end{array}$ & $\mathrm{p}$ \\
\hline Preterm Birth & $4(66.7 \%)$ & $9(90.0 \%)$ & .52 & $2(14.3 \%)$ & $3(33.3 \%)$ & .34 \\
Caesarean \% & $5(83.3 \%)$ & $8(80.0 \%)$ & 1.0 & $10(71.4 \%)$ & $7(77.8 \%)$ & 1.0 \\
Emerg C/S & $3(50.0 \%)$ & $6(60.0 \%)$ & 1.0 & $6(42.9 \%)$ & $4(44.4 \%)$ & 1.0 \\
Apgar 1 min $\leq 7$ & $3(50.0 \%)$ & $3(30.0 \%)$ & .61 & $5(35.7 \%)$ & $1(11.1 \%)$ & .34 \\
Apgar 5 min $\leq 7$ & $2(33.3 \%)$ & $0(0 \%)$ & .13 & $1(7.1 \%)$ & $0(0 \%)$ & 1.0 \\
NICU admit & $3(50.0 \%)$ & $4(40.0 \%)$ & 1.0 & $0(0 \%)$ & $1(11.1 \%)$ & .39 \\
SCN admit & $1(16.7 \%)$ & $4(40.0 \%)$ & .59 & $4(28.6 \%)$ & $3(33.3 \%)$ & 1.0 \\
\hline Note. SDB $=$ sleep-disordered breathing, PE = preeclampsia, GH = gestational hypertension, C/S $=$ \\
caesarean, NICU = neonatal intensive care unit, SCN = special care nursery. *1 PE excluded for CPAP \\
use
\end{tabular}


Table 5.16 Biomarkers of Fetal Growth in Cord Blood - Hypertensive Groups by SDB Status

\begin{tabular}{|c|c|c|c|c|c|c|c|c|c|}
\hline & \multicolumn{2}{|c|}{$\begin{array}{c}\text { PE } \\
(n=10)\end{array}$} & \multicolumn{3}{|c|}{$\begin{array}{c}\text { GH } \\
(n=20)\end{array}$} & \multicolumn{4}{|c|}{$\begin{array}{l}\text { Controls } \\
(n=26)\end{array}$} \\
\hline & $\begin{array}{c}\text { SDB } \\
(n=4)\end{array}$ & $\begin{array}{c}\text { No SDB } \\
\left(n=6^{a}\right)\end{array}$ & $P$ & $\begin{array}{c}\text { SDB } \\
(n=13)\end{array}$ & $\begin{array}{c}\text { No SDB } \\
(\mathrm{n}=7)\end{array}$ & $p$ & $\begin{array}{c}\text { SDB } \\
(n=8)\end{array}$ & $\begin{array}{l}\text { No SDB } \\
\left(\mathrm{n}=17^{\mathrm{b}}\right)\end{array}$ & $\mathrm{p}$ \\
\hline IGF-1 (pg/ml) & $\begin{array}{c}2009.2 \pm \\
2337.9\end{array}$ & $\begin{array}{c}1546.0 \pm \\
2177.3\end{array}$ & .76 & $\begin{array}{c}5940.4 \pm \\
3960.9\end{array}$ & $\begin{array}{c}6459.0 \pm \\
3268.4\end{array}$ & .77 & $\begin{array}{c}8976.7 \pm \\
2340.8\end{array}$ & $\begin{array}{c}5529.9 \pm \\
4171.3\end{array}$ & .015 \\
\hline IGF-2 (pg/ml) & $\begin{array}{c}52581.6 \pm \\
61432.0\end{array}$ & $\begin{array}{c}51621.1 \pm \\
56199.0\end{array}$ & .98 & $\begin{array}{c}98133.9 \pm \\
47881.8\end{array}$ & $\begin{array}{c}88067.6 \pm \\
28569.5\end{array}$ & .62 & $\begin{array}{c}118422.6 \pm \\
29792.0\end{array}$ & $\begin{array}{c}80448.3 \pm \\
37455.6\end{array}$ & .02 \\
\hline IGFBP-2 (ng/ml) & $173.7 \pm 45.4$ & $243.7 \pm 116.0$ & .29 & $179.4 \pm 98.4$ & $117.1 \pm 66.9$ & .15 & $97.1 \pm 50.4$ & $177.8 \pm 116.2$ & .024 \\
\hline Gestation (weeks) & $31.8 \pm 5.0$ & $32.7 \pm 4.0$ & .75 & $38.1 \pm 1.7$ & $37.4 \pm 3.1$ & .49 & $38.8 \pm 1.0$ & $39.4 \pm 1.2$ & .25 \\
\hline
\end{tabular}

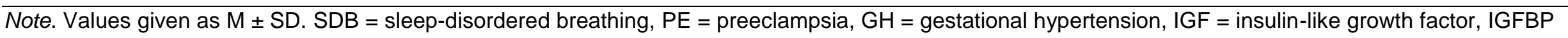
$=$ insulin-like growth factor binding protein.

a $\mathrm{n}=5$ for IGFBP-1

${ }^{b} \mathrm{n}=16$ for IGFBP-1 
Table 5.17 Biomarkers of Fetal Growth in Cord Blood across Hypertensive Groups

\begin{tabular}{lcccc}
\hline & $\begin{array}{c}\text { PE } \\
(\mathrm{n}=10)\end{array}$ & $\begin{array}{c}\mathrm{GH} \\
(\mathrm{n}=20)\end{array}$ & $\begin{array}{c}\text { Control } \\
(\mathrm{n}=26)\end{array}$ & $\mathrm{p}$ \\
\hline IGF-1 $(\mathrm{pg} / \mathrm{ml})$ & $1731.3 \pm 2124.3 \mathrm{a}$ & $6121.9 \pm 3653.3 \mathrm{~b}$ & $6632.9 \pm 3986.4 \mathrm{~b}$ & $.002^{\dagger}$ \\
IGF-2 $(\mathrm{pg} / \mathrm{ml})$ & $52005.3 \pm$ & $94610.7 \pm$ & $92600.1 \pm$ & $.029 \ddagger$ \\
& $54889.3 \mathrm{a}$ & $41593.5 \mathrm{~b}$ & $39000.3 \mathrm{~b}$ & \\
IGFBP-1 $(\mathrm{ng} / \mathrm{ml})$ & $24.2 \pm 14.0$ & $19.2 \pm 13.0$ & $15.2 \pm 8.9$ & .13 \\
IGFBP-2 $(\mathrm{ng} / \mathrm{ml})$ & $215.7 \pm 97.3$ & $157.6 \pm 92.0$ & $152.0 \pm 105.9$ & .22 \\
Gestation $(\mathrm{weeks})$ & $32.3 \pm 4.2 \mathrm{a}$ & $37.9 \pm 2.2 \mathrm{~b}$ & $39.2 \pm 1.2 \mathrm{~b}$ & $<.001$ \\
\hline
\end{tabular}

Note. Values given as $\mathrm{M} \pm \mathrm{SD}$. $\mathrm{PE}=$ preeclampsia, $\mathrm{GH}=$ gestational hypertension, IGF $=$ insulin-like growth factor, IGFBP = insulin-like growth factor binding protein. Means in the same row that do not share subscript differ at $\mathrm{p}<.05$ in the Newman-Keuls significant difference comparison.

${ }^{\dagger}$ no longer significant after ANCOVA with covariate of gestational age at sample, $p=.46$.

$\mp$ no longer significant after ANCOVA with covariate of gestational age at sample, $p=.46$. 
5.6 Supplementary Figures

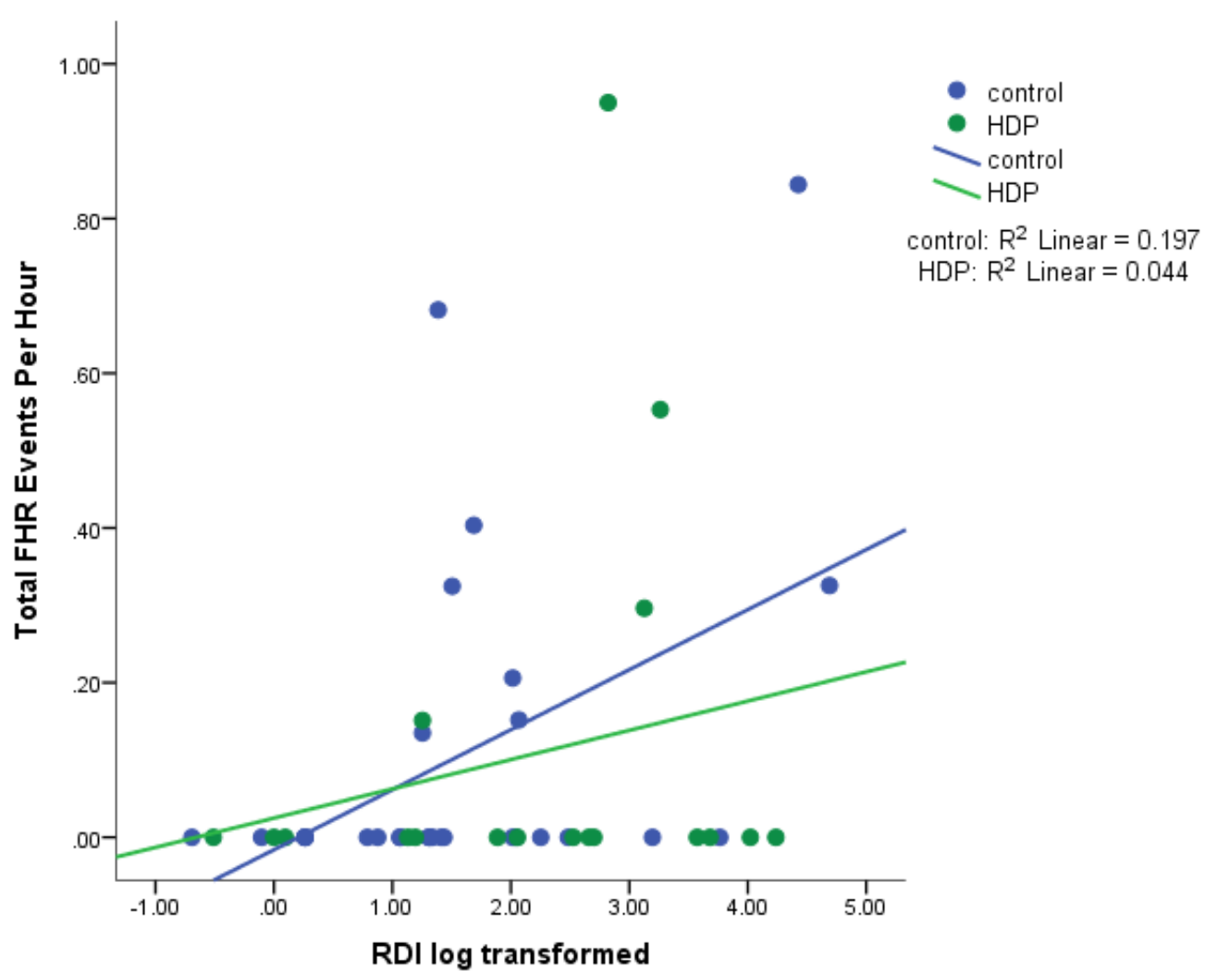

Figure 5.7 Scatterplot demonstrating relationship between RDI (log transformed) and total number of fetal heart rate (FHR) events on cardiotocography (CTG) per hour. A significant association exists in the control $(p=.02)$ but not the HDP group $(p=.40)$. HDP = hypertensive disorders of pregnancy, $R D I=$ respiratory disturbance index. 


\section{Chapter 6-}

\section{Periodic Limb Movements in Sleep During Pregnancy:}

\section{A Common but Benign Disorder?}

Manuscript accepted for publication on $7^{\text {th }}$ September 2017.

Wilson DL, Walker SP, Fung AM, O'Donoghue FJ, Barnes M, Howard ME. Periodic limb movements in sleep during pregnancy: a common but benign disorder. Sleep and Biological Rhythms, 2018, 16(1): 11-20. DOI: 10.1007/s41105-017-0125-7.

The final publication is available at http://link.springer.com/article/10.1007/s41105-017-0125-7 


\section{Preface - Chapter 6}

In the process of analysing the PSG studies for this project, it was noted that PLMS were common in this pregnant cohort. A review of the literature revealed that there is a distinct lack of data on the frequency of PLMS in normotensive and hypertensive pregnancy, despite ample focus on the frequency of RLS during pregnancy. In Chapter 6 we aimed to evaluate the presence of PLMS in late pregnancy and its relationship to HDP, and other clinical correlates.

This chapter was published as a manuscript in Sleep and Biological Rhythms and hence the style and format follow the specific requirements set out by the journal except where detailed in the main preface (page v). The author accepted version rather than the final published PDF version has been included for copyright reasons -

"This article is protected by copyright and all rights are held exclusively by Japanese Society of Sleep Research. The PDF version is not to be self-archived in electronic repositories. The author accepted manuscript version may be deposited in any repository, provided it is only made publicly available 12 months after official publication and provided acknowledgement is given to the original source of publication and a link is inserted to the published article on Springer's website."

This chapter was first submitted for publication whilst recruitment was still in process. The 15 participants with chronic hypertension originally recruited for this project were included in this manuscript, before a final decision had been made to exclude them from analyses in Chapters 3 through 5. 
Periodic Limb Movements in Sleep during Pregnancy - a Common but Benign Disorder?

Shortened title - PLMS during Pregnancy.

Danielle L. Wilson, MSc. ${ }^{1,2,3}$; Susan P. Walker, MBBS, MD, FRANZCOG, CMFM 2,4; Alison M. Fung, FRANZCOG, DDU²; Fergal J O'Donoghue, MB, BCh, PhD'1,3; Maree Barnes, MBBS 1,3; Mark E Howard, MBBS, FRACP, GDEB, PhD ${ }^{1,3}$.

${ }^{1}$ Institute for Breathing and Sleep, Austin Health, Heidelberg, Victoria, Australia;

2Department of Perinatal Medicine, Mercy Hospital for Women, Heidelberg, Victoria, Australia.

${ }^{3}$ Department of Medicine, University of Melbourne, Parkville, Victoria, Australia.

${ }^{4}$ Department of Obstetrics and Gynaecology, University of Melbourne, Parkville, Victoria, Australia.

Compliance with Ethical Standards -

All procedures performed in studies involving human participants were in accordance with the ethical standards of the Human Research Ethics Committees at Austin Health, Mercy Hospital for Women and the University of Melbourne in Melbourne, Victoria, Australia, and with the 1964 Helsinki declaration and its later amendments or comparable ethical standards. Informed consent was obtained from all individual participants included in the study.

This research was supported by the Austin Medical Research Foundation, the Australian Stillbirth Alliance, and the Medical Research Foundation for Women and Babies. Author D. Wilson was supported by a National Health and Medical Research Council (NHMRC) postgraduate scholarship.

Mark Howard receives research support from the Resmed Foundation, Philips Respironics and the Cooperative Research Centre (CRC) for Alertness, Safety and Productivity. Maree Barnes receives research support from AirLiquide Healthcare. This article is not related to either relationship. Danielle Wilson, Susan Walker, Alison Fung and Fergal O'Donoghue declare they have no conflict of interest.

Address correspondence to: Danielle L. Wilson, Ground Floor Bowen Centre, Institute for Breathing and Sleep, Austin Health, Heidelberg, Victoria, Australia. Tel: 6139496 3517; Fax: 6139496 5124; email: danielle.wilson@austin.org.au 


\begin{abstract}
Purpose - Pregnant women have a two-to-three-fold increased prevalence of Restless Legs Syndrome (RLS) compared to the general population, and the majority of RLS patients also experience Periodic Limb Movements in Sleep (PLMS). PLMS have been associated with sleep disturbance as well as autonomic heart rate and blood pressure responses, however the prevalence, cause and significance of PLMS during pregnancy remain unknown. This study evaluated the presence of PLMS in late pregnancy and its relationship to hypertensive disorders of pregnancy.

Methods - Ninety-one women in the third trimester of pregnancy underwent overnight polysomnography. An RLS questionnaire and the Multivariate Apnea Risk Index were administered and venous blood was sampled within 2 weeks of the sleep study.

Results - After exclusions due to obstructive sleep apnoea and signal loss, PLMS data was available for 73 women, 36 hypertensive women and $37 \mathrm{BMI}$-and gestation-matched controls. PLMS were found to be very common during pregnancy; $45 \%$ of women had a PLMS Index $>5$ and $25 \%$ had a PLMS Index $>15$. The number of PLMS per hour did not differ by hypertensive status. Sleep quality was mostly unaffected by PLMS, as was change in blood pressure overnight. While RLS was reported by $18.3 \%$ of the sample, this did not reliably predict the presence of PLMS. Despite iron deficiency being common in this population, it was not associated with PLMS.
\end{abstract}

Conclusions - This novel study investigating the frequency and impact of PLMS during pregnancy revealed that PLMS are very common in the third trimester, however this disorder appears to be benign in terms of objective sleep quality and relationship with pregnancy-related hypertension.

Keywords - leg movements, polysomnography, sleep quality, blood pressure, iron studies 


\subsection{Introduction}

During pregnancy, physiological changes and development of sleep disorders may impair sleep. In particular, Restless Legs Syndrome (RLS) affects women about twice as often as men, and may become worse or appear for the first time during pregnancy. ${ }^{22,455}$

RLS is a neurologic condition characterised by an urge to move, usually associated with paresthesia, that occurs or worsens at rest and is relieved by activity. ${ }^{456} \mathrm{~A}$ key characteristic of RLS is the worsening of symptoms in the evening and at night, with most patients reporting difficulty falling and staying asleep due to unpleasant leg sensations. As a consequence of disrupted sleep, excessive daytime fatigue and sleepiness often result. ${ }^{456}$

Pregnant women have a two to three times higher risk of RLS than the general population. 457 Prevalence estimates during pregnancy vary from $2.9 \%$ to $34 \% .{ }^{16,17,19,21,22,25}$ The prevalence of RLS symptoms often increases in the third trimester, ${ }^{25,458}$ with symptoms tending to resolve post-partum. ${ }^{16,19}$

Approximately $80 \%$ of patients with RLS also experience stereotyped repetitive movements once asleep, a condition known as periodic limb movements in sleep (PLMS). ${ }^{456,459}$ Periodic limb movement disorder (PLMD) is diagnosed when the number of PLMs per hour of sleep (PLMS Index; PLMSI) exceeds 15 movements per hour, and the associated sleep disruption causes excessive sleepiness or impairment in daytime functioning. ${ }^{459}$

Although the exact cause of PLMS is unknown, the pathophysiology of PLMS during pregnancy is thought to be similar to that of RLS. In the non-pregnant patient, the most accepted hypothesised mechanism of RLS is dopaminergic insufficiency. ${ }^{28}$ Well-recognised factors associated with RLS in pregnancy include iron and folate deficiencies. ${ }^{457,460}$

The clinical significance of PLMS has been a subject of debate. ${ }^{163} \mathrm{~A}$ stereotyped autonomic response accompanies each PLM, consisting of a rapid rise in heart rate and arterial blood pressure, followed by a significant and rapid bradycardia and a return of blood pressure to baseline values. Because patients can have several hundred PLMs every night, repetitive PLMS-related blood pressure surges during sleep could contribute to an increased risk of hypertensive disease ${ }^{29}$ or aggravate poor blood pressure control.

Despite the substantial interest in RLS during pregnancy there is a paucity of studies exploring PLMS in pregnancy, likely due to the requirement of polysomnography (PSG) for diagnosis. No studies have investigated the prevalence of PLMS during pregnancy, although they appear to be very common in multiple-gestation pregnancies and women with RLS in pregnancy. ${ }^{30,31}$

The aim of this study is to investigate how frequently PLMS occur in both normotensive and hypertensive pregnancy. Due to the autonomic changes accompanying PLMS, we hypothesise that 
PLMS are more common in women with hypertensive disease during pregnancy, and that PLMS may be associated with significant blood pressure changes during sleep. This study also examined whether RLS is a reliable predictor of PLMS in pregnancy, and whether PLMS severity correlates with serum measures of iron and folate.

\subsection{Materials and Methods}

\subsubsection{Study Participants}

Normotensive pregnant women and women with a hypertensive disorder of pregnancy (gestational hypertension, preeclampsia or chronic hypertension) were recruited to the study between October 2012 and May 2015. The Human Research Ethics Committees at Austin Health, Mercy Hospital for Women and University of Melbourne approved the study and informed consent was obtained from all participants.

Women with gestational hypertension and preeclampsia were recruited from the Pregnancy Day Assessment Centre or the Antenatal Ward, whereas participants with chronic hypertension and BMIand gestation-matched normotensive controls were recruited from the antenatal outpatient clinic at the Mercy Hospital for Women. Exclusion criteria included $<18$ years of age, multiparity gestation (i.e. twins), severe maternal or fetal comorbidities, and previous diagnosis of a sleep disorder.

\subsubsection{Investigational Measures}

Questionnaires were administered to assess symptoms of RLS and other sleep disorders. The diagnosis of RLS was based on the four essential diagnostic criteria for RLS, ${ }^{296}$ with all four of the essential criteria required to make the diagnosis of RLS. Questions regarding the frequency of symptoms and whether the symptoms were present prior to the current pregnancy were included (Appendix D).

The Multivariate Apnea Risk (MAP) Index ${ }^{112}$ (Appendix C) is based on a model developed for predicting the diagnosis of sleep apnoea. The full version includes 16 self-report symptoms from four domains associated with sleep disorders - sleep-disordered breathing, difficulty sleeping, excessive daytime sleepiness and narcolepsy-like symptoms. Frequency of each of the symptoms over the past month was scored as follows $-(0)=$ never, $(1)=$ less than once a week, $(2)=1-2$ times per week, $(3)=3-4$ times per week, and $(4)=5-7$ times per week.

\subsubsection{Polysomnography}

Overnight polysomnography (PSG) was conducted in either the Austin Health sleep laboratory using the Compumedics E series (Abbotsford, Victoria, Australia), or in the participant's home with the Somté (Compumedics, Abbotsford, Australia) portable sleep-monitoring device. Signals recorded both inlaboratory and at home included electroencephalogram (EEG; with recommended derivations of F4, 
$C 4$ and $\mathrm{O} 2$ referenced to $M 1$ ), electrooculogram (EOG), electromyogram (EMG), electrocardiogram (ECG), arterial oxygen saturation, thoracic and abdominal respiratory effort via inductance plethysmography, nasal airflow measured via nasal canula, oronasal thermistor, bilateral leg EMG of the anterior tibialis, snoring and body position. After sensors were placed and calibrated, signal quality and impedances were checked. Recordings were analysed by a single experienced sleep technologist who was blinded to all patient details. Respiratory events were scored as per the most recent AASM criteria at the time of the study. ${ }^{461}$ Leg movements classified as PLMS were visually scored as per the AASM criteria, ${ }^{38}$ and the PLMS Index (PLMSI) was calculated as the number of PLMS per hour of sleep. A leg movement was not scored if it occurred during a period from $0.5 \mathrm{sec}$ proceeding or following an apnoea or hypopnoea. ${ }^{38}$

\subsubsection{Blood sampling}

Maternal venous blood samples were assayed for Iron Studies (iron, transferrin, saturation, and ferritin), red blood cell (RBC) folate, haematocrit and haemoglobin.

\subsubsection{Procedures}

PSG was performed in the third trimester of pregnancy. Inpatients in the antenatal ward required monitoring with the portable device. A trained sleep technologist with over 10 years of experience (DW) was responsible for setting up all sleep recordings. Immediately prior to and immediately following the PSG, blood pressure was measured at the upper arm in the seated position using an automated blood pressure monitor (Omron HEM-7203). Height and weight was also recorded at this time. Within \pm 2 weeks of PSG, the participants completed the RLS Questionnaire and MAP Index and a venous blood sample was taken.

\subsubsection{Statistical Analysis}

All statistical analyses were performed with SPSS 21.0 (SPSS Inc., Chicago, Illinois) and Stata 12.0 (StataCorp, Texas). Values are given in means with standard deviations $(M \pm S D)$ or median and interquartile range (Mdn (IQR)) for non-normally distributed variables. A two-sided $p$ value of less than 0.05 was considered to indicate statistical significance.

A cut-off of PLMSI $>15 / \mathrm{hr}$ was used based on current definitions. ${ }^{459}$ Leg movements often occur in association with termination of respiratory events; therefore participants with significant OSA (RDI > 20) confounding the presence of PLMS were excluded from data analysis.

Between group comparisons were made using chi square test for independence for categorical variables, independent t-tests (two tailed) for normally-distributed continuous variables and MannWhitney $\mathrm{U}$ tests or Kruskal-Wallis tests for non-normally distributed continuous variables. 
Logistic regression was used to assess the univariate relationships between PLMS classified as a dichotomous variable (defined as a PLMSI > 15/hr) and demographic and symptom-related factors from the questionnaires, and odds ratios (ORs) were calculated. Stepwise logistic regression analysis was performed with PLMSI > 15/hr as the dependent variable, and explanatory variables with an $\alpha$ of less than 0.10 on univariate analysis were included.

\subsection{Results}

\subsubsection{Participants}

A total of 91 participants were recruited and underwent polysomnography. Three participants had poor leg signals, and two did not have leg sensors on PSG due to safety concerns (both were inpatients considered as a falls risk). Thirteen participants were excluded from PSG analysis due to OSA (RDI > 20; eight with gestational hypertension or preeclampsia, one with chronic hypertension and four normotensive controls), leaving a total of 73 participants with PLMS assessable on PSG. Twenty-six participants chose to have an in-laboratory sleep study and 47 chose a home sleep study. There were no differences in PLMS/hr of sleep and PLMSI > 15/hr ( $p=.14$ and .82 respectively).

On average, the women in the study were 33.5 years old $(S D=4.4)$ and had the PSG performed at a gestational age of $33.4(3.1)$ weeks. The mean pre-pregnancy BMI of the participants was $31.4 \mathrm{~kg} / \mathrm{m}^{2}$ (7.0), which increased to $36.2 \mathrm{~kg} / \mathrm{m}^{2}(6.4)$ at the time of the PSG. Just over half $(56 \%)$ of the sample was nulliparous, 35 (38.5\%) women had gestational hypertension or preeclampsia, and $14(15.4 \%)$ women had chronic hypertension.

\subsubsection{Periodic Limb Movements during Sleep}

Overall, almost half of all pregnant women had a PLMSI > 5/hr, with a quarter having a PLMSI > 15/hr (Table 6.1). The number of PLMS/hr did not differ across groups according to hypertensive status, or during different sleep stages. Within the hypertensive group, PLMS/hr of sleep also did not differ between the women with gestational hypertension/preeclampsia and chronic hypertension (Mdn (IQR) $=2.9(0.0,12.3)$ vs. $1.4(0.0,24.2), p=.99)$. The difference in blood pressure at the start to the end of the night was not affected by PLMS status, in fact there was a trend for those with PLMSI > 15/hr to have a slight fall in systolic $(p=.08)$ and diastolic blood pressure $(p=.08)$ overnight compared to those without PLMS (Figure 6.1). 
Table 6.1 PLMS Indices for Normotensive and Hypertensive Participants

\begin{tabular}{lcccc}
\hline Mdn (IQR) & $\begin{array}{c}\text { Normotensive } \\
(\mathrm{n}=37)\end{array}$ & $\begin{array}{c}\text { Hypertensive } \\
(\mathrm{n}=36)\end{array}$ & $\begin{array}{c}\text { Overall } \\
(\mathrm{n}=73)\end{array}$ & p value \\
\hline PLMS/hr of sleep & $4.7(0.5,21.8)$ & $2.9(0.0,14.7)$ & $4.0(0.0,18.2)$ & .29 \\
PLMS/hr of NREM & $5.6(0.6,27.2)$ & $2.3(0.0,17.8)$ & $4.8(0.0,22.4)$ & .29 \\
PLMS/hr of REM & $0.0(0.0,0.0)$ & $0.0(0.0,0.0)$ & $0.0(0.0,0.0)$ & .99 \\
PLMSI > 5/hr & $18(48.6 \%)$ & $15(41.7 \%)$ & $33(45.2 \%)$ & .72 \\
PLMSI > 15/hr & $10(27.0 \%)$ & $8(22.2 \%)$ & $18(24.7 \%)$ & .84 \\
\hline
\end{tabular}

Note. PLMS = periodic limb movements in sleep; PLMSI = periodic limb movements in sleep index.

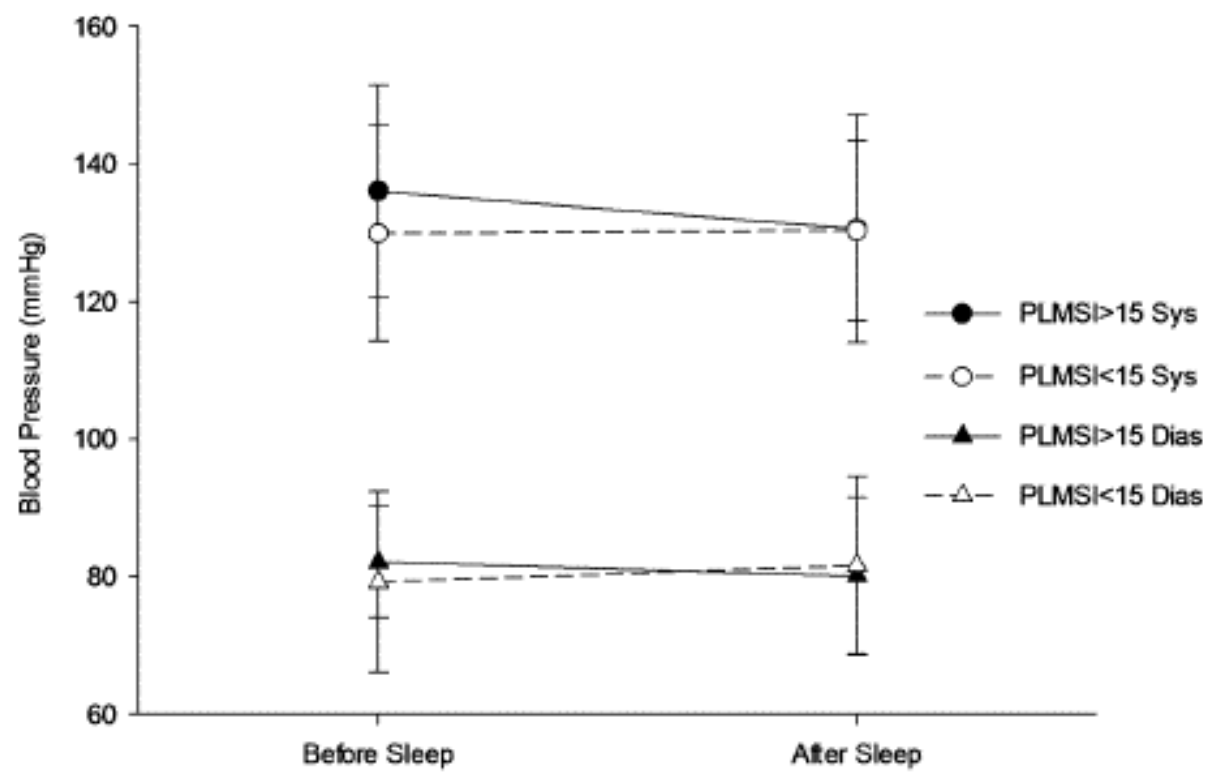

Figure 6.1 Systolic (Sys) and diastolic (Dias) blood pressure $(M \pm S D$ ) measured prior to bedtime and upon morning awakening. PLMSI = Periodic Limb Movements in Sleep Index

As shown in Table 6.2, the overall sleep quality of those with a PLMSI > 15/hr was not different to those without PLMS, except for a trend towards a significantly greater percentage of sleep time spent in REM sleep and a trend towards more cortical arousals. There was no difference in RDI between the two groups. 


\begin{tabular}{lccc}
\hline & \multicolumn{2}{c}{ PLMSI $>15 / h r$} & \\
& $(\mathrm{n}=18)$ & No & p value \\
\hline Arousal Index & $26.8 \pm 13.5$ & $20.4 \pm 6.2$ & .06 \\
Sleep Efficiency & $80.3 \pm 9.8$ & $80.1 \pm 9.8$ & .96 \\
Sleep Latency & $20.2 \pm 22.2$ & $13.2 \pm 10.2$ & .21 \\
Awakenings & $26.9 \pm 7.3$ & $26.0 \pm 10.1$ & .74 \\
Awake Time & $77.9 \pm 38.7$ & $83.3 \pm 50.1$ & .68 \\
\% TST REM sleep & $17.8 \pm 5.4$ & $15.1 \pm 5.1$ & .06 \\
\% TST N3 sleep & $27.4 \pm 9.1$ & $33.0 \pm 13.0$ & .10 \\
RDI/hr & $3.7(1.7,6.3)$ & $3.5(1.9,7.7)$ & .56
\end{tabular}

Note. $\mathrm{PLMSI}=$ periodic limb movements in sleep index; \%TST = percentage of total sleep time; RDI = Respiratory Disturbance Index.

Iron studies and RBC folate was available for 43 participants with valid PLMS measurement on PSG. There were no differences on the iron studies results between those with and without PLMSI >15/hr cut off (Table 6.3). However, women with a PLMSI of $>15 / \mathrm{hr}$ had significantly lower haemoglobin and a trend towards lower hematocrit levels.

Table 6.3 PLMS and Iron Studies

\begin{tabular}{lccc}
\hline & \multicolumn{2}{c}{ PLMSI $>15 / \mathrm{hr}$} & \\
& $(\mathrm{n}=15)$ & No & p value \\
& $14.4 \pm 6.5$ & $13.5 \pm 5.5$ & .64 \\
\hline Iron (umol/L) & $3.8 \pm 0.8$ & $4.0 \pm 0.5$ & .57 \\
Transferrin (g/L) & $15.9 \pm 7.3$ & $14.0 \pm 6.3$ & .38 \\
Saturation (\%) & $17.0(14.0,42.0)$ & $21.5(11.3,50.3)$ & .95 \\
Ferritin (ug/L) & $3009.8 \pm 782.5$ & $2659.8 \pm 485.6$ & .14 \\
RBC Folate (nmol/L) & $117.2 \pm 9.9$ & $126.0 \pm 10.6$ & .03 \\
Hb (g/L) & $0.35 \pm .03$ & $0.37 \pm .03$ & .06 \\
HCT (L/L) & & 03
\end{tabular}

Note. PLMSI = periodic limb movements in sleep index; RBC = red blood cell; $\mathrm{Hb}=$ haemoglobin; $\mathrm{HCT}$ $=$ hematocrit. 


\subsubsection{Restless Legs Syndrome Questionnaire}

The RLS questionnaire was completed by 71 participants. Each of the four essential features of RLS were endorsed by approximately one third of the participants, and $13(18.3 \%)$ women endorsed all four symptoms to meet the diagnostic criteria of RLS. There was no significant difference for RLS prevalence across hypertensive groups (normotensive $=19.4 \%$ vs. GH/PE $=19.2 \%$ vs. Ess $H T N=11.1 \% ; p=.84$ ).

Of those with RLS, six (46.2\%) participants reported that their symptoms occur at least twice a week with the rest reporting symptoms less than twice a week. Five (38.5\%) reported that their symptoms caused them distress or impairment of functioning. Six (46.2\%) women reported RLS symptoms prior to their current pregnancy.

\subsubsection{Predictors of PLMS}

When all demographic variables, RLS criteria and measures of sleep disturbance from the MAP Index were subject to univariate analysis, the only factor associated with an increased risk of PLMSI > 15/hr was Question 5 on the MAPI - "During the last month, have you had or been told about tossing, turning or thrashing while asleep" (see Supporting Table 6.5). A stepwise selection model identified Question 5 on the MAPI and the frequency of RLS symptoms as the strongest independent predictors of a PLMSI $>15 /$ hr during pregnancy $\left(x^{2}=9.47, p=.009, R^{2}=0.16\right.$; Table 6.4).

Ten of the women meeting RLS diagnostic criteria also had valid PLMS assessment on PSG. Only three $(30 \%)$ of these women had a PLMSI $>15$, and five $(50 \%)$ had a PLMSI of $>5$.

Table 6.4 Factors associated with PLMSI > 15/hr during Pregnancy on Stepwise Logistic Regression Model

\begin{tabular}{lccc}
\hline Variable & Coefficient & OR $(95 \%$ Cl) & p value \\
\hline MAPI 5 - tossing \& turning & 0.82 & $2.28(1.01-5.17)^{\mathrm{a}}$ & .04 \\
RLS frequency $^{*}$ & 0.81 & $2.25(0.95-5.34)^{\mathrm{b}}$ & .065 \\
Constant & -4.31 & $.01(6.76 \mathrm{e}-4-0.27)$ & .005
\end{tabular}

${ }^{*}$ OR for ordinal variables indicate the change in odds for an increase of one category respectively.

aCategories for frequency were 0) never, 1) rarely, less than once a week, 2) 1-2 times per week, 3) 3-

4 times per week, 4) 5-7 times per week.

${ }^{b}$ Categories for frequency were 0 ) never, 1) less than twice a week, 2) at least twice a week. 


\subsection{Discussion}

This is the first study to examine the prevalence of PLMs in pregnancy, and their association with hypertensive disorders of pregnancy, objectively confirmed sleep disturbance and symptoms of RLS. We found that PLMS are very common in both normotensive and hypertensive pregnancies. During the third trimester of pregnancy $45 \%$ of women had a PLMSI of $>5$ and $25 \%$ had a PLMSI $>15$. Importantly, this study did not identify that PLMS were associated with adverse outcome, either in relation to hypertensive status, or daytime symptoms.

The high prevalence of PLMS during late pregnancy observed in this study is in contrast to a previous study examining objectively-measured PLMS in the non-pregnant population, which showed a prevalence of $7.6 \%$ for a PLMSI $>15 .{ }^{462}$ Another study showed the presence of PLMS was very rare before the age of 40.463 In contrast, the most recently reported prevalence of PLMSI>15 for women was high at $26 \% .464$ However, this study only included women over 40 and demonstrated that PLMS were significantly related to increasing age.

The clinical significance of PLMS remains unclear. Some, but not all studies ${ }^{465-467}$ have suggested important associations between PLMS and PSG variables such as sleep stage fragmentation ${ }^{468}$ and sleep efficiency, ${ }^{469}$ and subjective symptoms of sleepiness and sleep disturbance. ${ }^{462}$ It might be expected that frequent PLMS would correlate with impaired sleep quality particularly in pregnant women where sleep disturbance is more common. In our sample, PSG indices of sleep quality were mostly unaffected by the presence of PLMS. Although those with a PLMSI > 15 tended to have more cortical arousal from sleep, they did not spend any less time awake during the night and had a higher proportion of REM sleep. We found that symptoms of sleep disruption and excessive sleepiness were not associated with PLMS in this large cohort of pregnant women.

PLMS are associated with significant fluctuations in heart rate and blood pressure, ${ }^{470}$ with the implication that these repetitive hemodynamic changes overnight may impact on the development of cardiovascular disease. Due to the requirement of PSG to diagnose PLMS, most studies have focused on the association between RLS and hypertension 471,472 or cardiovascular disease. ${ }^{26}$ Three studies have shown that PLMS are more common in people with essential hypertension compared to the general population, ${ }^{464,473}$ with the likelihood of hypertension increasing with PLMS severity by as much as 2-fold for those with a PLMSI > 30.29 PLMS have been reported to be associated with increased nocturnal blood pressure in patients with insomnia, ${ }^{474}$ and more severe cardiovascular outcomes such as congestive heart failure ${ }^{475,476}$ and cardiac mortality. 477 Although biologically plausible, no studies have actually confirmed a causal relationship between PLMS and essential hypertension. ${ }^{478}$

Importantly, this study did not demonstrate any association with PLMS or RLS and hypertensive disease in pregnancy. Reassuringly, even women with a PLMSI > 15/hr trended towards lower systolic and diastolic BP across the night. While these findings appear contradictory to the non-pregnant literature, we studied a group of young women while the majority of studies looking at PLMS and cardiovascular 
outcomes focus on the elderly, as the prevalence of PLMS increases with advanced age. ${ }^{479}$ Any cardiovascular changes resulting from PLMS are likely to be chronic and develop over many years, while the transient changes in pregnancy- while very substantial- may not have been present long enough to result downstream physiological effects.

This study also found the prevalence of RLS in the third trimester of pregnancy to be higher than in the general population. We found that $18.3 \%$ women met criteria for RLS, compared to an estimated 5$10 \%$ in the general population. ${ }^{456}$ Almost half of those with RLS reported experiencing symptoms prior to their current pregnancy, compared to a Brazilian study reporting that $90 \%$ of those with RLS had their symptoms start during pregnancy. ${ }^{22}$ Despite studies suggesting that PLMS are present in approximately $80 \%$ of RLS cases ${ }^{26,479}$ we found that only $50 \%$ of women meeting the diagnostic criteria for RLS also had a PLMSI > 5. We found the best predictor of PLMS was not RLS but the frequency of RLS symptoms and the MAPI symptom regarding "tossing, turning or thrashing while asleep". While these were the strongest independent predictors in a stepwise selection model, these two items still only explained $16 \%$ of the variance.

The cause of PLMS is still unknown, ${ }^{163}$ and very little data exists on the pathophysiology associated with PLMS. Due to their similarities, many authors have focused on what causes RLS instead. In nonpregnant patients, the prevailing hypothesis of mechanism of RLS is dopaminergic insufficiency. ${ }^{28}$ Evidence has also been found that iron deficiency in the CNS, specifically reduced ferritin and elevated transferrin levels in the cerebrospinal fluid despite normal peripheral iron status, may be involved in primary RLS. ${ }^{480}$

The association between pregnancy and increased prevalence of RLS is not fully understood, however it has been hypothesised that iron and folate deficiencies are causative factors. ${ }^{16,21,460}$ Levels of haemoglobin and ferritin can fall in the second half of pregnancy, regardless of taking iron and vitamin supplements, due to fetal growth and hemodilution. ${ }^{16}$ However, a number of experimental studies have not supported these associations between RLS and low haemoglobin levels, serum iron, iron-binding capacity, ferritin, iron saturation and folic acid. ${ }^{17,19,31,458,464}$

There are no studies in pregnancy investigating iron and folate deficiencies with PLMS. Our study has demonstrated no differences in serum measures of iron, transferrin, saturation, ferritin or RBC folate in those with or without PLMS. Despite this, the PLMSI > 15/hr group had lower haemoglobin and hematocrit levels compared to those with a PLMSI $<15$.

Despite no association with PLMS, our results suggested that iron deficiency was common with ferritin levels below $75 \mathrm{ug} / \mathrm{L}$ in $88 \%$ of the sample, a level at which oral iron supplementation may be considered for RLS during pregnancy. ${ }^{481}$ This is despite the majority of pregnant patients taking prenatal vitamins containing iron supplementation. 
Limitations of this study are that the generalisability of the results of this study may be limited by the study population. Given the participants were from a study investigating hypertensive disorders of pregnancy and BMI matched controls, a high number of women were either overweight or obese prior to pregnancy. However, we did not find any relationship between BMI and frequency of PLMS. Possible selection bias may also be a limitation of this study as those with sleep concerns may have been more likely to consent to the study, however potential participants were informed that the study was about sleep during pregnancy with no specific mention of PLMS or RLS.

We used the gold standard of full PSG to measure PLMS in this study, however like many studies in this field ${ }^{462,464,465,474}$ each participant was monitored for one night only. While night-to-night variability in severity of PLMS has been demonstrated, the PLMS index often does not change systematically from the first to the second night ${ }^{482-484}$ indicating that the overall prevalence of PLMS would likely remain reasonably stable. Diagnostically, there is no requirement for multiple nights of recording to demonstrate PLMS for PLMD. 38,459

Despite being the largest study to report on PLMS within a pregnant sample, this study and its conclusion regarding no association between PLMS and hypertension may be limited by sample size. In the most recent study of PLMS prevalence, ${ }^{464} 37.6 \%$ of hypertensive participants had a PLMSI>15 compared to $22 \%$ of the normotensive participants. In order to find this magnitude of effect with a power of $80 \%$ at alpha $=0.05$, we would have required approximately 130 participants per group.

RLS was diagnosed using a questionnaire rather than by face-to-face interview. A false positive diagnosis rate of up to $16 \%$ has been shown with use of the four key diagnostic features only, suggesting a limitation with diagnostic instruments based on subject report without further differential diagnosis to exclude confounding conditions. ${ }^{485}$ The diagnostic criteria for RLS was updated by the International Restless Legs Syndrome Study Group (IRLSSG) in 2014 to add a fifth essential criterion to exclude the presence of mimics and improve specificity of diagnosis. ${ }^{486}$ Prevalence of RLS and the relationship between RLS and PLMS may be affected by the use of the 2007 diagnostic criteria. ${ }^{38}$ As much of the data was collected for this study before the criteria was updated, we can only acknowledge this as a potential limitation to our study and something to be considered for future research in this area.

This was the first study to investigate the frequency and impact of PLMS during pregnancy. Our results demonstrate that PLMS are very common in the third trimester, with a prevalence at least 3-times that reported in the general population, and that PLMS were frequently present without RLS. Despite this, this study provides reassuring data that this disorder appears to be benign in pregnancy, with no adverse impact on objective sleep measures, sleepiness or changes in blood pressure from evening to morning. Further, the lack of association with specific symptoms indicates that many pregnant women are not even aware of their PLMS. Our findings add to the current controversy regarding the importance of PLMS - are they a clinically significant problem or simply an epiphenomenon. ${ }^{478,487}$ 


\subsection{Acknowledgment}

The authors would like to thank the staff at the antenatal clinics at Mercy Hospital for Women and the Sleep Laboratory at Austin Health for their support of this project. In particular, thank you to Gabrielle Pell for assisting with participant recruitment and Pavlina Toman for polysomnograph analysis. We appreciate the valuable contribution made by each of the research participants.

This research was supported by the Austin Medical Research Foundation, the Institute for Breathing and Sleep, the Australian Stillbirth Alliance, and the Medical Research Foundation for Women and Babies. Author D. Wilson was supported by a National Health and Medical Research Council (NHMRC) postgraduate scholarship. 


\subsection{Supporting Table}

Table 6.5 Demographic and RLS Symptoms associated with PLMSI > 15/hr during Pregnancy

\begin{tabular}{|c|c|c|c|}
\hline Variable & $\begin{array}{l}\text { Units or Category } \\
\text { (Range/SD) }\end{array}$ & OR $(95 \% \mathrm{Cl})$ & $p$ value \\
\hline Age $^{*}$ & $\operatorname{yr}(23-42 / 4.4)$ & $1.25(0.72-2.16)$ & .43 \\
\hline Parity & Times given birth (0-4) & $0.99(0.58-1.71)$ & .99 \\
\hline BMI at sleep study* & $\mathrm{kg} / \mathrm{m}^{2}(23.1-53.1 / 5.8)$ & $1.14(0.67-1.94)$ & .64 \\
\hline Gestation at sleep study* & weeks $(23+5-37+4 / 3.1)$ & $1.63(0.84-3.16)$ & .15 \\
\hline RLS diagnosis & Yes or No & $1.40(0.31-6.36)$ & .66 \\
\hline RLS criteria 1 & Yes or No & $1.73(0.50-6.00)$ & .39 \\
\hline RLS criteria 2 & Yes or No & $2.10(0.56-7.83)$ & .27 \\
\hline RLS criteria 3 & Yes or No & $1.83(0.50-6.74)$ & .36 \\
\hline RLS criteria 4 & Yes or No & $2.48(0.69-8.84)$ & .16 \\
\hline RLS Frequency* & Frequency $(0-2)^{a}$ & $1.93(0.88-4.20)$ & .10 \\
\hline RLS prior to pregnancy & Yes or No & $1.59(0.34-7.44)$ & .56 \\
\hline MAPI4 - frequent awakenings* & Frequency $(0-4)^{b}$ & $1.24(0.80-1.93)$ & .34 \\
\hline MAPI5 - tossing and turning ${ }^{*}$ & Frequency $(0-4)^{\mathrm{b}}$ & $2.31(1.20-4.46)$ & .01 \\
\hline MAPI6 - difficulty falling asleep* & Frequency $(0-4)^{b}$ & $0.81(0.52-1.26)$ & .36 \\
\hline MAPI7 - legs jumpy and jerky* & Frequency $(0-4)^{b}$ & $1.08(0.63-1.86)$ & .77 \\
\hline MAPI11 - excessive sleepiness ${ }^{*}$ & Frequency $(0-4)^{b}$ & $0.91(0.57-1.46)$ & .71 \\
\hline MAPI15 - morning fatigue* & Frequency $(0-4)^{b}$ & $0.98(0.62-1.55)$ & .94 \\
\hline
\end{tabular}

Note. $\mathrm{N}=73 . \mathrm{OR}=$ odds ratio; $\mathrm{BMI}=$ body mass index; $\mathrm{GH}=$ gestational hypertension.

*OR for continuous and ordinal variables indicate the change in odds for an increase of one standard deviation or one category respectively.

aCategories for frequency were 0) never, 1) less than twice a week, 2) at least twice a week.

${ }^{\mathrm{b} C}$ Categories for frequency were 0) never, 1) rarely, less than once a week, 2) 1-2 times per week, 3) 34 times per week, 4) 5-7 times per week. 
Chapter 7 - General Discussion

Sleep-Disordered Breathing in Hypertensive Disorders of Pregnancy:

Impact on Maternal and Fetal Outcomes 
The primary aim of this thesis was to investigate the prevalence of SDB in HDP, and secondarily to determine the acute and chronic effects of SDB on maternal and fetal health. We also aimed to investigate how frequently PLMS occur in both normotensive and hypertensive pregnancy. This thesis was submitted following an approved alternative format which allows the inclusion of published manuscripts, and therefore each empirical chapter contains its own detailed discussion. The following section provides a summary of the major findings of this thesis and the implications of these, followed by limitations of the current study and directions for future research.

\subsection{Summary of Research Findings}

Hypertensive disorders of pregnancy are the most common and serious medical disorders in pregnancy and are a leading contributor to maternal and perinatal mortality and morbidity. ${ }^{1-3}$ SDB is a risk factor for hypertension in non-pregnant populations. Although a similar association has been described in pregnancy the confounding effect of obesity has not been accounted for in most studies. In an attempt to address this gap in the literature, in Chapter 3 we hypothesised that pregnant women with HDP (both $\mathrm{PE}$ and $\mathrm{GH}$ ) would have a higher rate of SDB compared to BMI- and gestation-matched normotensive pregnant women.

The key finding of Chapter 3 was that mild SDB occurred in more than half of women with HDP, however over a third of BMI-matched controls also had mild SDB, with no increase in the overall median RDI in the HDP group. However, we identified a 2 -fold increase in moderate and severe SDB in the hypertensive group, suggesting that more severe SDB may play an important role in the development of hypertension in pregnancy. Our results clearly indicate that obesity is an important confounder in the relationship between HDP and SDB and failing to adjust for this will overestimate the strength of this association.

Given the prevalence of SDB in both HDP and normotensive obese pregnant women, Chapter 4 focused on identifying how SDB may influence the course of hypertensive disease during pregnancy and the potential physiological mechanisms underpinning this relationship. The main findings in Chapter 4 were presented separately for the PE, GH and normotensive groups. Within the PE and GH groups, the presence of SDB did not alter the clinical course of the disease, with no differences found in any measure of hypertensive disease severity between those with and without SDB. Unexpectedly, those with GH and SDB had lower systolic and diastolic BP across the second and third trimester of pregnancy compared to those without SDB. Importantly, the presence of SDB did not worsen indicators of organ dysfunction or anti-angiogenic markers typically associated with PE.

Within the normotensive controls, those with SDB were no more likely to develop HDP than those without SDB. BP across pregnancy was unaffected apart from the last week of gestation, when there was a sharp rise in systolic BP within the SDB group. Surprisingly, there was some evidence for an increase in anti-angiogenic markers within those who did not have SDB. 
Lastly, increasing the severity threshold of SDB to a higher level $(R D I \geq 15)$ did not alter these findings. In fact, compared to women without SDB, those with PE and SDB delivered their babies at a later gestation, and those with GH and SDB had lower systolic BP on the night of the sleep study. Two biomarkers of hypertension severity, ET-1 and sEng, were lower in those with HDP and SDB. Given the low incidence of moderate to severe SDB in this study however, we were inadequately powered to draw firm conclusions regarding these findings.

Given that hypertensive disease during pregnancy is associated with fetal compromise, it is important to identify whether these already at-risk fetuses will be further disadvantaged by concurrent maternal SDB. The aim of Chapter 5 was to clarify whether the presence of SDB influenced the acute and chronic health of the fetus in women with HDP, as well as in normotensive women.

The key finding from Chapter 5 was that maternal SDB had no detrimental impact on fetal wellbeing. In particular, within the HDP group, signs of nocturnal fetal distress on continuous CTG were uncommon, and unrelated to maternal respiratory events. Although there was a moderate positive relationship between severity of SDB and fetal heart rate decelerations in the normotensive women, these fetal heart rate events were mild. SDB was not associated with slowed third trimester fetal growth or FGR at birth, and hormonal indicators of fetal growth in cord blood were not impacted upon by SDB within either the GH or PE groups. In fact, the neonates of women with HDP and SDB were larger than those with HDP and no SDB, and within the control group it was the participants with SDB who had higher IGF-1 and IGF-2, and lower IGFBP-2 levels, which are typically associated with fetal growth and placental development. In terms of perinatal outcomes, SDB did not affect gestation and mode of delivery, Apgar scores indicating fetal condition at birth, or neonatal admission for higher care needs.

Further, there was no evidence to suggest that more severe degrees of SDB and maternal hypoxaemia have any detrimental effect on fetal growth or perinatal outcomes. Similar to the maternal outcomes in Chapter 4, those with moderate to severe SDB in fact had a better outlook in some respects, such as higher customised birth centiles, with higher IGF-2 and lower IGFBP-1 levels; both associated with enhanced fetal growth and placental development. Again, sample size was not adequate to draw firm conclusions for some analyses, given the low frequency of adverse perinatal outcomes, particularly within the control group (i.e. no preterm births and no NICU admissions), and the low rate of severe SDB.

Lastly, there is an absence of data on the frequency of PLMS in normotensive and hypertensive pregnancy. In Chapter 6 we investigated how frequently PLMS occur, and the potential correlates and consequence of this condition. Our key findings were that PLMS are very common in the third trimester of pregnancy, however this disorder appears to be benign. Specifically, almost half of all pregnant women had a mild degree of PLMS (PLMSI $>5 / h r$ ) and a quarter were more severely affected (PLMSI $>15 / \mathrm{hr}$ ). The prevalence of PLMS did not differ across hypertensive groups, and nocturnal BP was not affected by PLMS status. The presence of PLMS did not impact on overall sleep quality, apart from a 
trend towards a significantly greater percentage of sleep time in REM sleep and more cortical arousals. Interestingly, there were no differences in the iron studies results between those with and without PLMS. Reported presence of RLS was not predictive of PLMS, rather the strongest factor associated with an increased risk of PLMS was self-reported "tossing and turning while asleep".

\subsection{Implications of the Studies}

This study has identified that the prevalence of at least mild SDB is very high among women with PE and $\mathrm{GH}$, and higher than may have been previously reported in BMI-matched normotensive women. Although the rate of moderate to severe SDB was relatively low overall, it was higher than that observed in non-pregnant female populations. ${ }^{41,264}$ In Chapter 3 we addressed the feasibility of offering treatment for SDB to the high proportion of obese and hypertensive pregnant women experiencing this condition. There are currently no recommendations for treatment of SDB in pregnancy. Some authors have suggested that treatment should be given to all women with mild, moderate and severe SDB given the absence of pregnancy-specific data, ${ }^{488}$ whereas others suggest that an important goal should be to abolish oxygen desaturations below $90 \%{ }^{489}$ Otherwise, it has been proposed that pregnant women are treated as per the AASM guidelines for the general population - treatment for SDB is recommended for an RDI $\geq 15$ regardless of symptoms, or an RDI of $5-14$ if associated with EDS, loud snoring, witnessed apnoeas or awakenings. ${ }^{302}$

The lack of association between SDB and adverse outcomes for the mother and infant in our study helps inform this debate. Our study suggests that treatment for all pregnant women diagnosed with any degree of SDB is neither feasible (given the large numbers involved) and any benefit is likely to be modest. Even if it were feasible to screen and treat affected women, adherence to CPAP may be poor. In our study, all women with mild SDB were offered clinical review with a sleep physician, with strong recommendations for the 15 women with moderate to severe SDB to attend follow-up. From this, only three women accepted CPAP treatment. This problem has been identified in other studies ${ }^{263}$ and suggests that stronger links between SDB and poor pregnancy outcomes may need to be demonstrated before pregnant women see treatment as a high priority.

As yet there are no published clinical trials assessing the efficacy of CPAP during pregnancy, and a number of small cohort studies in women with chronic hypertension or PE risk factors have had mixed results. ${ }^{272,273}$ One of the women with PE in our study was diagnosed with very severe SDB (RDI of 150/hr) and was excluded from analysis following commencement of CPAP treatment. Our published case study following her CPAP treatment found improvements in BP and many markers of PE severity and potentially prolonged her pregnancy for five weeks allowing significant gain in gestation and size. ${ }^{158}$ It seems likely that there is a threshold at which treatment of SDB will translate into maternal and fetal benefits, but this awaits clarification in future research.

While data regarding the benefit of CPAP to improve pregnancy outcomes is lacking, pregnant women should still be recommended treatment for SDB for the same indications as apply to the general 
population - daytime sleepiness and quality of life, risk of cardiovascular events in the long term, risk of depression and increased risk of motor vehicle accidents. ${ }^{82}$

This study has clarified the role that obesity plays in the relationship between SDB and pregnancyrelated hypertensive disease. Mild SDB was present in over a third of our normotensive obese women, with the prevalence of at least moderate SDB at $12.5 \%$ - well above that reported in healthy weight normotensive pregnant women $(0-6 \%)_{.8,259,260}$ Obesity is a strong risk factor for SDB, ${ }^{123,264}$ and predisposes to HDP through increased sympathetic activation, inflammation and oxidative stress contributing to placental ischaemia and production of anti-angiogenic factors. ${ }^{3,490}$ Our BMI-matched study design meant there were no significant differences in BMI between the SDB and No SDB groups when comparing both maternal and fetal outcomes, but nonetheless it was considered as a confounder when relevant. Interestingly, the degree of obesity was not associated with measures of disease severity within the PE or GH groups, such as days between HDP diagnosis and delivery and BP across pregnancy.

Obesity is also a strong risk factor for poor perinatal outcomes, particularly stillbirth. ${ }^{491,492}$ If SDB was identified as an intermediary in the relationship between obesity and poor fetal outcomes, it would provide a potential therapeutic avenue to prevent fetal complications, however our results did not support such a link. Any study investigating a role for SDB in the multifactorial causes of HDP, fetal compromise and stillbirth needs to strongly consider the interplay between obesity and these factors.

Future studies need to address what criteria and degree of SDB are most important in terms of pregnancy health outcomes. SDB can be measured in numerous ways - more concrete indices that have recommended scoring guidelines such as $\mathrm{AHI}, \mathrm{RDI}$, and $\mathrm{ODI},{ }^{295}$ versus 'softer' measures of SDB including snoring and upper airway flow limitation which do not have validated analysis guidelines.

Gender differences in SDB disease manifestation exist, with studies suggesting that females are more likely to have partial upper airway obstruction or UARS rather than discrete respiratory events such as obstructive apnoeas. ${ }^{117-119}$ Flow limitation in the absence of sleep apnoea is more common in pregnancy compared to non-pregnant women, and particularly in women diagnosed with PE. ${ }^{21,284,369}$ Furthermore, a small cluster of studies have suggested that CPAP treatment of upper airway flow limitation in PE appears to result in improved BP and cardiac output ${ }^{169,270}$ and improved fetal movements, ${ }^{284}$ suggesting that even mild levels of SDB involving upper airflow limitation in HDP may be clinically important. 8,270,284 Clinical trials are eagerly awaited.

A challenge in applying such criteria is reproducibility of assessment. For Chapter 3 of our study we attempted to measure flow limitation, with the hypothesis that this would be more prevalent in the HDP group compared to normotensive women. However, we found that between one experienced sleep scientist and two senior sleep physicians, and despite methodologies detailed in previous studies, ${ }^{369,493,494}$ our concordance was variable and ranged from $27-81 \%$ agreement on the number 
of flow limited breaths in a random sample of 40 epochs, across only four PSG studies. Given the poor reproducibility of this measure we did not include it in our analysis. Although a number of automated positive airway pressure (PAP) machines now contain internal algorithms to measure flow limitation in order to adjust pressure according to the user's respiration, none of these have been validated for research use either.

Despite advances in perinatal care, the prevalence of PE remains unchanged. ${ }^{3}$ There has been no substantial improvement in prevention of this disorder, ${ }^{330,495}$ and the only cure is delivery of the fetus and placenta. In this study, we hoped to identify a novel modifiable risk factor (SDB) for PE. It is likely that for any prospective treatment to be successful it needs to start early in the pregnancy to alter pathogenesis. ${ }^{496} \mathrm{~A}$ common hypothesis regarding the development of PE is the two-step model (as discussed in detail in Chapter 4), which implies that there are two distinct phases at which SDB could influence the development of PE during pregnancy. At the placentation stage very early in pregnancy, it would be those women with pre-existing SDB resulting in hypoxaemia, oxidative stress and inflammation whose SDB would have an impact upon abnormal implantation and vascular remodelling. By this reasoning, we would expect those with pre-existing SDB to be more likely to develop early-onset or severe PE, rather than those who develop SDB later in pregnancy. In turn, treatment of SDB may theoretically play an important role in preventing or lessening abnormal placental development.

The second stage of PE development and the way in which SDB may impact upon this involves the amplification of oxidative stress, inflammation and the release of anti-angiogenic factors which lead to the maternal syndrome of PE. At this later stage, it could be hypothesised that SDB emerging during pregnancy may amplify hypertensive disease rather than being a causative factor. The aim of SDB treatments such as CPAP in this scenario would be to ameliorate the endothelial damage and prolong pregnancy to a safer gestation rather than prevent the disease entirely.

Despite these theoretical arguments, our results showed that SDB did not have a detrimental effect on the course of hypertensive disease during pregnancy, although we did find that moderate to severe SDB was more common in those with already established GH and PE. Our cross-sectional study design however did not allow attribution of causality. It would be valuable in future studies to longitudinally follow pregnant women with pre-existing SDB and BMI-matched controls to observe the impact of exposure to the apnoea/hypoxia/regeneration cycle for the duration of pregnancy.

One curious result from this study was that hypertensive women with more severe SDB seemed to have a better outlook than those without SDB, as evidence by a longer gestation from diagnosis and larger customised birthweight centiles. While this is likely a chance finding, it is interesting to consider whether HDP associated with SDB may have a more benign course. The intermittent hypoxic challenge of SDB may result in less placental ischaemia, release of anti-angiogenic molecules and endothelial dysfunction than the chronic placental dysfunction and hypoxia/ischaemia classically associated with PE. HDP linked to SDB would see placental and maternal endothelial re-oxygenation daily, whereas 
PE resultant from early uteroplacental insufficiency would be chronically hypoxic. We acknowledge this is speculative but may warrant further investigation. This could be through well-designed animal models and adequately powered prospective studies, with careful attention to potential confounding variables, and comprehensive clinical and laboratory outputs.

In Chapter 6, we found that PLMS were a very common but rarely researched phenomenon during pregnancy. We found no association with specific symptoms of RLS suggesting that many pregnant women are not even aware of their PLMS. The clinical significance of PLMS in pregnancy remains uncertain, but the lack of associations previously found between PLMS and hypertension, ${ }^{29,464}$ or PLMS and sleep fragmentation ${ }^{468,469}$ in other populations suggests that treatment of PLMS during pregnancy is unlikely to improve clinical outcomes.

On the other hand, almost 1 in 5 women endorsed symptoms indicating the presence of RLS, with over a third of these women reporting that their RLS symptoms caused them distress. There is no reported data on using pharmacological agents for treatment of RLS or PLMS during pregnancy, however the mainstay of RLS therapy is dopaminergic agents. ${ }^{28}$ If possible, pharmacological treatment should be avoided and the woman informed that RLS and PLMS symptoms usually disappear or become much better after delivery. ${ }^{497}$ Conservative treatments during pregnancy include avoidance of caffeine, correction of electrolyte abnormalities, and administration of folate supplements if folate deficiency is present. ${ }^{498}$

\subsection{Limitations of the Current Thesis}

The limitations specific to each empirical chapter have been discussed in detail, with the most notable limitations common across the study as a whole.

Firstly, our power calculation for sample size was adequate for our primary hypothesis that SDB would be more common in HDP compared to normotensive pregnancies (Chapter 3), although this was based on a lower expected prevalence of SDB in the control group guided by previous studies which largely involved non-BMI matched women. For Chapters 4 and 5, we had comprehensive maternal and fetal outcomes but this limited our capacity to recruit larger numbers of participants. The sample size for comparisons was reduced when the decision was made to split the GH and PE women into separate groups for analysis due to differing clinical features and outcomes. Although we had a very high success rate collecting PSG data, there was a degree of missing data for nocturnal CTG and third trimester ultrasound (mostly within the PE group), and maternal and cord blood samples at delivery. This was generally beyond our control but would have impacted on the power to assess relationships between fetal outcomes and SDB.

A larger sample size would have strengthened our results, and provided more participants with moderate to severe SDB to explore whether more severe degrees of SDB are related to fetal and maternal outcomes. For example, to determine whether moderate to severe SDB is related to a two- 
fold increase in FGR at birth (which was 15.4\% within the normotensive group), we would require 117 participants with and without moderate to severe SDB in each group (providing a power of $80 \%$ with an alpha of 0.05 ). Given our prevalence of moderate to severe SDB was $12.5 \%$ in the normotensive sample, an overall sample size of 936 participants with SDB would be required. It would be more achievable to find an effect of more severe SDB within the HDP sample. We found that $40 \%$ of fetuses of mothers with HDP but no SDB had fetal heart rate events overnight. If a doubling in prevalence of CTG abnormalities was deemed significant in those with moderate to severe SDB, we would have required 23 participants in each group - which extrapolates to 102 per group with or without SDB overall given a prevalence of $22.5 \%$ for moderate to severe SDB in HDP. This sample size would also give sufficient power (> $80 \%$ ) to find that those with PE or GH and concurrent SDB deliver their infants sooner following diagnosis of HDP (i.e. pregnancy prolonged for half the number of days from HDP diagnosis to delivery), indicating greater disease severity. These sample size calculations are based on the assumption that SDB is pathological, however for the main part our findings were not in the hypothesised directions and a larger sample may not have altered our conclusions that SDB was not detrimental to maternal or fetal health.

Secondly, as with most studies looking at SDB and pregnancy outcomes, the severity level of SDB in our study was generally mild. The theory of shared pathophysiology between SDB and PE, and the proposition that maternal hypoxaemia leads to fetal hypoxia and subsequent fetal compromise, highly depends on significant oxygen desaturations following maternal apnoea. Most studies relating SDB to hypertension in the non-pregnant population have shown a dose-response relationship, ${ }^{65,365,386}$ with a recent review study concluding that there is no evidence of increased cardiovascular risk from mild SDB. ${ }^{74}$ Other studies have indicated that the degree of nocturnal hypoxaemia rather than number of respiratory events was more closely associated with sympathetic activation, daytime BP and hypertension. ${ }^{394,395}$ The overall median RDI of our sample of women with SDB was 11.4/hr, however the ODI3\% and ODI $4 \%$ were quite low at only 4.6 and $1.9 / \mathrm{hr}$ respectively. This low degree of maternal hypoxaemia may partly explain why SDB in pregnancy was of little clinical significance. However, we did have $18 \%$ of our overall sample with an $\mathrm{RDI} \geq 15$, which is a high proportion compared to previous studies in pregnancy ${ }^{7,123}$ and allowed us to look at the impact of more severe disease.

Another explanation for our largely negative findings is that pregnancy is a transient condition of only nine months. Many studies finding that SDB is related to hypertension and cardiac disease have followed participants for many years post initial assessment ${ }^{65,366,371}$ giving the detrimental effects of the apnoea/hypoxia/reoxygenation cycle adequate time to produce pathological changes. Temporary changes in SDB during pregnancy may not have been present long enough to result in significant downstream cardiovascular effects, particularly given the usual cardiovascular adaptations that accompany pregnancy (increased cardiac output and decreased peripheral resistance), and the adaptive capacity of the fetus to withstand hypoxic insults. 
We used gold standard full PSG to measure SDB and PLMS in this study. However, since PSG was conducted on one occasion, we cannot ascertain whether these conditions predated the pregnancy or when in pregnancy they developed. It is unlikely the degree of SDB or PLMS stays completely static across pregnancy, however it is very difficult to perform full PSG multiple times across pregnancy. Two studies have measured SDB in the first and third trimester of pregnancy with an increase in severity across gestation, however the magnitude of this change was minor. ${ }^{7,122}$ Ascribing SDB status as measured in the third trimester as a surrogate for SDB across pregnancy was our best option and should still allow for detection of differences if they existed. It is also likely that those with moderate and severe SDB would have had it prior to pregnancy or at least from the early stages, and hence our persistent negative results when defining SDB at higher levels of severity should be more representative of the chronic effects of SDB on maternal and fetal wellbeing. In regards to our PLMS analysis, there is no requirement for multiple nights of recording to demonstrate PLMS for diagnosis of PLMD, ${ }^{38,459}$ with studies showing that the PLMS index often does not change systematically from the first to the second night of recording. ${ }^{482-484}$

Despite these limitations, the novel outcomes of our study add useful data to the existing literature, particularly as many of our results disputed our a priori hypotheses and raise questions about the conclusions made to date in this field.

\subsection{Directions for Future Research}

This study strengthens previous work demonstrating the relationship between SDB and PE and GH, but demonstrates the importance of adjusting for the confounding effect of obesity. Matching for BMI demonstrated that the relationship appears to be restricted to more severe SDB. A key question remains as to what degree of SDB and which indices (i.e. snoring, flow limitation, number or severity of apnoeic episodes vs. oxygen desaturations) are most strongly related to poor maternal and fetal outcomes, if at all. Prospective longitudinal studies that have found no association between SDB and poor outcomes have typically had milder levels of SDB within their samples ${ }^{7,263}$ and are likely underpowered to determine associations with more severe levels of disease. ${ }^{123}$ Also, many women in these studies did not manifest SDB until the third trimester of pregnancy. These results contrast with retrospective studies looking at women with pre-pregnancy and more substantial SDB that have found associations with higher risk for PE, preterm birth and small for gestational age infants, ${ }^{262,280}$ which suggests that preexisting and/or more severe SDB may represent a greater hazard for adverse outcomes during pregnancy. One consideration is that ethically it may be difficult to explore a relationship involving untreated severe SDB during pregnancy as treatment is indicated in the maternal interest, irrespective of the potential impact on perinatal outcomes. Even our results may have been diluted by the exclusion of the three women with the most severe SDB commencing CPAP following PSG. The only foreseeable way around this may be to compare women who take up treatment versus those who refuse or have poor adherence, but this will introduce its own bias requiring care in the interpretation of results. 
Much of the literature in this field suggests that the next steps are adequately powered intervention robust trials of CPAP treatment for women with SDB during pregnancy. Despite our study identifying no increased risk in adverse maternal or fetal outcomes related to SDB, we did find that women with a gestational hypertensive disease were twice as likely to have moderate to severe SDB. A number of small studies have suggested that CPAP may play a role in improved haemodynamics and pregnancy outcomes for women with chronic hypertension and PE. 158,169,270,273,284 Well designed and executed trials will inform recommendations for CPAP as a prevention or treatment for SDB-related pregnancy complications.

Our original study design included pregnant women diagnosed with chronic hypertension, however recruitment of these women was discontinued due to the low SDB prevalence we found in our small cohort of $n=15$, impacting the feasibility of obtaining an adequate sample size to find any effects. The literature would suggest this is unusual, as studies looking at SDB during pregnancy often find a relatively high proportion of women with SDB have pre-existing hypertension ${ }^{260,499}$ and consider it as a confounder. ${ }^{123,262}$ Further expansion in this area assessing the outcomes for those with comorbid chronic hypertension and SDB remains important, especially given that those with chronic hypertension who develop superimposed PE have particularly poor outcomes. ${ }^{148,149}$

Another interesting area that fell outside the scope of this thesis, is the impact that maternal sleep position has on fetal health. Pregnant women are often advised to avoid sleeping on their back, following observational data suggesting a higher risk of stillbirth associated with back sleeping. ${ }^{283,500}$ The suggested rationale is that the uterus may compress the inferior vena cava and, to a lesser extent, the aorta, causing a decrease in the blood return to the heart and cardiac output. ${ }^{501}$ However, SDB may also play a role as it is more pronounced in the supine position, 502 as it was in this study (Figure 3.3). Despite this, on average our participants still spent approximately a quarter of their sleep time in the supine position (see Table 3.4).

Pregnant women who lie in the supine position may develop syncopal symptoms, however only $4 \%$ of patients in five studies of maternal sleep position had pre-syncopal symptoms related to aortocaval occlusion in a supine position, ${ }^{503-507}$ and there was no evidence of fetal compromise. On the other hand, a gradient effect of maternal body position on cardiac output has been demonstrated, with the greatest reductions in cardiac output in the supine followed by the right then left lateral positions. ${ }^{508}$ Also, compared to the left-sided position, the adverse effects of lying in the supine position include decreased uterine blood flow ${ }^{509}$ and reduced pulsatility index in the fetal middle cerebral artery, a surrogate marker of fetal hypoxia. ${ }^{510}$ Most recently, two studies into sleep-related risk factors for stillbirth have suggested that women who reportedly slept on their back or right side on the night before stillbirth were more likely to experience a late stillbirth compared with women who slept on their left side. ${ }^{283,500}$ A significant limitation of these studies is a lack of maternal sleep position validation; the correlation between selfreport and video-determined left-side sleep time has been reported as only moderate $(r=.42)$, with a high degree of individual variability. ${ }^{511}$ The current literature does not appear strong enough to give 
evidence-based recommendations of maternal sleep position during pregnancy. Building upon our current data with objective measurement of sleep position and comprehensive acute and chronic fetal outcomes will assist us as we investigate this in further research of our own.

\subsection{Conclusion}

Our study was the first to look at the prevalence of SDB in HDP compared to a closely BMI- and gestation- matched normotensive control group, and to investigate how SDB may amplify the already heightened risk factors for adverse maternal and fetal outcomes in HDP. It was unique in that it closely examined in detail the acute and chronic effects that SDB may have on pathophysiological changes associated with negative outcomes, including nocturnal and longitudinal maternal BP across pregnancy, biochemical and angiogenic factors associated with HDP severity, nocturnal fetal distress on CTG, third trimester fetal growth trajectory and insulin markers of fetal growth in cord blood.

We found that SDB occurs in half of women diagnosed with a gestational hypertensive disorder but also in over a third of normotensive women of similar body habitus, suggesting that the previously reported relationship between SDB and hypertension in pregnancy is due at least in part to the confounding effect of obesity. A significant independent association between HDP and moderate SDB suggests that SDB may still play an important role in the development of hypertension during pregnancy. We observed that the presence of SDB did not influence the course of hypertensive disease in pregnancy, regardless of severity level, while acknowledging the limited power for analyses of severe SDB. Similarly, comorbid SDB on top of HDP did not have a detrimental effect on our indicators of fetal health and wellbeing, in both hypertensive and normotensive pregnant women. PLMS is another frequently observed sleep condition of pregnancy, with a prevalence at least 3-times that reported in the general population, however our data suggests this condition is benign in pregnancy.

The high prevalence of SDB in this population raises the question of the optimal definition and threshold for SDB in pregnancy, particularly given we found no adverse impact on maternal and fetal outcomes. Given the discrepancies between our results and others, this field requires further study to elucidate the exact pathways that connect SDB, HDP and perinatal outcomes. Some of these answers will be found in well-designed animal models and prospective longitudinal cohort studies. These latter studies should focus on women with more severe levels of SDB, where any benefit of intervention is likely to be greatest, and those with SDB preceding the pregnancy where the pathophysiological consequences play out across gestation. Data from these high-risk groups will best inform the design, conduct and expected effect size of interventional clinical trials involving CPAP to improve pregnancy outcomes. 


\section{Reference List}

1. Khan KS, Wojdyla D, Say L, Gülmezoglu AM, Van Look PF. WHO analysis of causes of maternal death: a systematic review. Lancet 2006;367(9516):1066-1074.

2. Redman CW, Sargent IL. Latest advances in understanding preeclampsia. Science. 2005;308(5728):1592-1594.

3. Sibai B, Dekker G, Kupferminc M. Pre-eclampsia. Lancet. 2005;365(9461):785-799.

4. Young T, Nieto FJ, Javaheri S. Systemic and pulmonary hypertension in obstructive sleep apnea. In: Kryger M, Roth T, Dement WC, eds. Principles and Practice of Sleep Medicine. 5th ed. Philadelphia, Pennsylvania: W. B. Saunders, 2011:1381-1392.

5. Shahar E, Whitney CW, Redline S, et al. Sleep-disordered breathing and cardiovascular disease: crosssectional results of the sleep heart health study. Am J Respir Crit Care Med. 2001;163(1):19-25.

6. Franklin KA, Holmgren P, Jonsson F, Poromaa N, Stenlund H, Svanborg E. Snoring, pregnancyinduced hypertension, and growth retardation of the fetus. Chest. 2000;117(1):137-141.

7. Pien G, Pack Al, Jackson N, Maislin G, Macones GA, Schwab RJ. Risk factors for sleep-disordered breathing in pregnancy. Thorax. 2014;69(4):371-377.

8. Reid J, Skomro R, Cotton D, et al. Pregnant women with gestational hypertension may have a higher frequency of sleep disordered breathing. Sleep. 2011;34(8):1033-1038.

9. Bourjeily G, Raker CA, Chalhoub M, Miller MA. Pregnancy and fetal outcomes of symptoms of sleepdisordered breathing. Eur Respir J. 2010;36(4):849-855.

10. Mindell JA, Jacobson BJ. Sleep disturbances during pregnancy. J Obstet Gynecol Neonatal Nurs. 2000;29(6):590-597.

11. Ko HS, Shin JC, Kim MY, et al. Sleep disturbances in Korean pregnant and postpartum women. J Psychosom Obstet Gynaecol. 2012;33(2):85-90.

12. Facco FL, Kramer J, Ho KH, Zee PC, Grobman WA. Sleep disturbances in pregnancy. Obstet Gynecol. 2010;115(1):77-83.

13. Baratte-Beebe K, Lee KA. Sources of midsleep awakenings in childbearing women. Clin Nurs Res. 1999;8(4):386-397.

14. Hutchison BL, Stone PR, McCowan LME, Stewart AW, Thompson JMD, Mitchell EA. A postal survey of maternal sleep in late pregnancy. BMC Pregnancy Childbirth. 2012;12:144.

15. Ford ES, Cunningham TJ, Giles WH, Croft JB. Trends in insomnia and excessive daytime sleepiness among US adults from 2002 to 2012. Sleep Med. 2015;16(3):372-378.

16. Manconi M, Govoni V, De Vito A, et al. Restless legs syndrome and pregnancy. Neurology. 2004;63(6):1065-1069.

17. Tunç T, Karadag YS, Dogulu F, Inan LE. Predisposing factors of restless legs syndrome in pregnancy. Mov Disord. 2007;22(5):627-631.

18. Balendran J, Champion D, Jaaniste T, Welsh A. A common sleep disorder in pregnancy: restless legs syndrome and its predictors. Aust N Z J Obstet Gynaecol. 2011;51(3):262-264.

19. Uglane MT, Westad S, Backe B. Restless legs syndrome in pregnancy is a frequent disorder with a good prognosis. Acta Obstet Gynecol Scand. 2011;90(9):1046-1048.

20. Ismailogullari S, Ozturk A, Mazicioglu MM, Serin S, Gultekin M, Aksu M. Restless legs syndrome and pregnancy in Kayseri, Turkey: a hospital based survey. Sleep Biol Rhythms. 2010;8(2):137-143.

21. Chen $\mathrm{P}$, Liou $\mathrm{K}$, Chen $\mathrm{C}$, Cheng $\mathrm{S}$. Risk factors and prevalence rates of restless legs syndrome among pregnant women in Taiwan. Sleep Med. 2012;13(9):1153-1157.

22. Alves DA, Carvalho LB, Morais JF, Prado GF. Restless legs syndrome during pregnancy in Brazilian women. Sleep Med. 2010;11(10):1049-1054.

23. Montplaisir J, Nicolas A, Godbout R, Walters AS. Restless legs syndrome and periodic limb movement disorder. In: Kryger M, Roth T, Dement WC, eds. Principles and Practice of Sleep Medicine. 3rd ed. Philadelphia, Pennsylvania: W. B. Saunders Company, 2000.

24. Neau JP, Porcheron A, Mathis S, et al. Restless legs syndrome and pregnancy: a questionnaire study in the poitiers district, France. Eur Neurol. 2010;64(5):268-274.

25. Harano S, Ohida T, Kaneita $\mathrm{Y}$, et al. Prevalence of restless legs syndrome with pregnancy and the relationship with sleep disorders in the Japanese large population. Sleep Biol Rhythms. 2008;6(2):102109.

26. Winkelman JW, Shahar ES, Sharief I, Gottlieb DJ. Association of restless legs syndrome and cardiovascular disease in the Sleep Heart Health Study. Neurology. 2008;70(1):35-42.

27. Winkelman JW, Finn L, Young T. Prevalence and correlates of restless legs syndrome symptoms in the Wisconsin Sleep Cohort. Sleep Med. 2006;7(7):545-52. 
28. Lam K, D'Ambrosio CM. Restless legs syndrome and periodic limb movement disorder in pregnancy. In: Bourjeily G, Rosene-Montella K, eds. Pulmonary Problems in Pregnancy: Springer, 2009:135-42.

29. Billars L, Hicks A, Bliwise D, et al. Hypertension risk and PLMS in restless legs syndrome. Sleep. 2007;30:A297-A298.

30. Nikkola E, Ekblad U, Ekholm E, Mikola H, Polo O. Sleep in multiple pregnancy: breathing patterns, oxygenation, and periodic leg movements. Am J Obstet Gynecol. 1996;174(5):1622-1625.

31. Dzaja A, Wehrle R, Lancel M, Pollmacher T. Elevated estradiol plasma levels in women with restless legs during pregnancy. Sleep. 2009;32(2):169-174.

32. American Academy of Sleep Medicine. International Classification of Sleep Disorders, $2^{\text {nd }}$ ed. Diagnostic and Coding Manual. Westchester, IL: American Academy of Sleep Medicine, 2005.

33. Li C, Hoffstein V. Snoring. In: Kryger M, Roth T, Dement WC, eds. Principles and Practice of Sleep Medicine. 5th ed. Philadelphia, Pennsylvania: W. B. Saunders, 2011.

34. Partinen M, Hublin C. Epidemiology of sleep disorders. In: Kryger M, Roth T, Dement WC, eds. Principles and Practice of Sleep Medicine. 5th ed. Philadelphia, Pennsylvania: W. B. Saunders, 2011.

35. Weiss JW, Remsburg S, Garpestad E, Ringler J, Sparrow D, Parker JA. Hemodynamic consequences of obstructive sleep apnea. Sleep. 1996;19(5):388-397.

36. Chervin RD. Use of clinical tools and tests in sleep medicine. In: Kryger M, Roth T, Dement WC, eds. Principles and Practice of Sleep Medicine. 5th ed. Philadelphia, Pennsylvania: W. B. Saunders, 2011.

37. Hirshkowitz M, Kryger M. Monitoring techniques for evaluating suspected sleep-disordered breathing In: Kryger M, Roth T, Dement WC, eds. Principles and Practice of Sleep Medicine. 5th ed. Philadelphia, Pennsylvania: W. B. Saunders, 2011.

38. Iber C, Ancoli-Israel S, Chesson A, Quan S, for the American Academy of Sleep Medicine. The AASM Manual for the Scoring of Sleep and Associated Events: Rules, Terminology and Technical Specifications. 1st ed. Westchester, III: American Academy of Sleep Medicine, 2007.

39. Peigneux $\mathrm{P}$, Laureys $\mathrm{S}$, Delbeuck X, Maquet P. Sleeping brain, learning brain. The role of sleep for memory systems. Neuroreport. 2001;12(18):A111-A124.

40. Young T, Palta M, Dempsey J, Skatrud J, Weber S, Badr S. The occurrence of sleep-disordered breathing among middle-aged adults. N Engl J Med. 1993;328(17):1230-1235.

41. Bixler EO, Vgontzas AN, Lin HM, et al. Prevalence of sleep-disordered breathing in women: effects of gender. Am J Respir Crit Care Med. 2001;163(3 Pt 1):608-613.

42. Vaughn BV, D'Cruz OF. Cardinal manifestations of sleep disorders. In: Kryger M, Roth T, Dement WC, eds. Principles and Practice of Sleep Medicine. 5th ed. Philadelphia, Pennsylvania: W. B. Saunders, 2011.

43. Mokhlesi B, Punjabi NM. "REM-related" obstructive sleep apnea: an epiphenomenon or a clinically important entity? Sleep. 2012;35(1):5-7.

44. Koo BB, Dostal J, loachimescu O, Budur K. The effects of gender and age on REM-related sleepdisordered breathing. Sleep Breath. 2008;12(3):259-264.

45. Eiseman NA, Westover MB, Ellenbogen JM, Bianchi MT. The impact of body posture and sleep stages on sleep apnea severity in adults. J Clin Sleep Med. 2012;8(6):655-666.

46. O'Connor C, Thornley KS, Hanly PJ. Gender differences in the polysomnographic features of obstructive sleep apnea. Am J Respir Crit Care Med. 2000;161(5):1465-1472.

47. Menon A, Kumar M. Influence of body position on severity of obstructive sleep apnea: a systematic review. ISRN Otolaryngol. 2013; 670381.

48. Oksenberg A, Silverberg DS. The effect of body posture on sleep-related breathing disorders: facts and therapeutic implications. Sleep Med Rev. 1998;2(3):139-162.

49. Fung AM, Wilson DL, Barnes M, Walker SP. Obstructive sleep apnoea and pregnancy: the effect on perinatal outcomes. J Perinatol. 2012;32(6):399-406.

50. Garcia-Rio F, Racionero MA, Pino JM, et al. Sleep apnea and hypertension: the role of peripheral chemoreceptors and the sympathetic system. Chest. 2000;117(5):1417-1425.

51. Smith ML, Niedermaier ON, Hardy SM, Decker MJ, Strohl KP. Role of hypoxemia in sleep apneainduced sympathoexcitation. J Auton Nerv Syst. 1996;56(3):184-190.

52. Cooper V, Pearson S, Bowker C, Elliott M, Hainsworth R. Interaction of chemoreceptor and baroreceptor reflexes by hypoxia and hypercapnia-a mechanism for promoting hypertension in obstructive sleep apnoea. J Physiol. 2005;568(2):677-687.

53. Carlson JT, Rångemark $\mathrm{C}$, Hedner JA. Attenuated endothelium-dependent vascular relaxation in patients with sleep apnoea. J Hypertens. 1996;14(5):577-584.

54. Shamsuzzaman AS, Winnicki M, Lanfranchi $P$, et al. Elevated C-reactive protein in patients with obstructive sleep apnea. Circulation. 2002;105(21):2462-2464. 
55. Steiropoulos P, Kotsianidis I, Nena E, et al. Long-term effect of continuous positive airway pressure therapy on inflammation markers of patients with obstructive sleep apnea syndrome. Sleep. 2009;32(4):537-543.

56. Yokoe T, Minoguchi K, Matsuo H, et al. Elevated levels of C-reactive protein and interleukin-6 in patients with obstructive sleep apnea syndrome are decreased by nasal continuous positive airway pressure. Circulation. 2003;107(8):1129-1134.

57. Canto GDL, Pachêco-Pereira C, Aydinoz S, Major PW, Flores-Mir C, Gozal D. Biomarkers associated with obstructive sleep apnea: a scoping review. Sleep Med Rev. 2015;23:28-45.

58. Tokuda F, Sando Y, Matsui H, Koike H, Yokoyama T. Serum levels of adipocytokines, adiponectin and leptin, in patients with obstructive sleep apnea syndrome. Intern Med. 2008;47(21):1843-1849.

59. Wallace AM, McMahon AD, Packard CJ, et al. Plasma leptin and the risk of cardiovascular disease in the west of Scotland coronary prevention study (WOSCOPS). Circulation. 2001;104(25):3052-3056.

60. Lam J, Xu A, Tam S, et al. Hypoadiponectinemia is related to sympathetic activation and severity of obstructive sleep apnea. Sleep. 2008;31(12):1721-1727.

61. Nakagawa $\mathrm{Y}$, Kishida K, Kihara S, et al. Nocturnal reduction in circulating adiponectin concentrations related to hypoxic stress in severe obstructive sleep apnea-hypopnea syndrome. Am J Physiol Endocrinol Metab. 2008;294(4):E778-E784.

62. Marshall NS, Wong KK, Cullen SR, Knuiman MW, Grunstein RR. Sleep apnea and 20-year follow-up for all-cause mortality, stroke, and cancer incidence and mortality in the Busselton Health Study cohort. J Clin Sleep Med. 2014;10(4):355-362.

63. Punjabi NM, Caffo BS, Goodwin JL, et al. Sleep-disordered breathing and mortality: a prospective cohort study. PLoS Med. 2009;6(8):e1000132.

64. Young T, Finn L, Peppard PE, et al. Sleep disordered breathing and mortality: eighteen-year follow-up of the wisconsin sleep cohort. Sleep. 2008;31(8):1071-1078.

65. Peppard PE, Young T, Palta M, Skatrud J. Prospective study of the association between sleepdisordered breathing and hypertension. N Engl J Med. 2000;342(19):1378-1384.

66. Zamarron C, Gude F, Otero Otero Y, Rodriguez-Suarez JR. Snoring and myocardial infarction: a 4-year follow-up study. Respir Med. 1999;93(2):108-112.

67. Hu FB, Willett WC, Manson JE, et al. Snoring and risk of cardiovascular disease in women. J Am Coll Cardiol. 2000;35(2):308-313.

68. D'Alessandro R, Magelli C, Camberini G, et al. Snoring every night as a risk factor for myocardial infarction: a case-control study. BMJ. 1990;300(6739):1557-1558.

69. Gottlieb DJ, Yenokyan G, Newman AB, et al. Prospective study of obstructive sleep apnea and incident coronary heart disease and heart failure: the Sleep Heart Health Study. Circulation. 2010;122(4):352360.

70. Javaheri S. Sleep disorders in systolic heart failure: a prospective study of 100 male patients. The final report. Int J Cardiol. 2006;106(1):21-28.

71. Redline S, Yenokyan G, Gottlieb DJ, et al. Obstructive sleep apnea-hypopnea and incident stroke: the Sleep Heart Health Study. Am J Respir Crit Care Med. 2010;182(2):269-277.

72. Punjabi NM, Ahmed MM, Polotsky VY, Beamer BA, O'Donnell CP. Sleep-disordered breathing, glucose intolerance, and insulin resistance. Respir Physiol Neurobiol. 2003;136(2-3):167-178.

73. Tasali E, Mokhlesi B, Van Cauter E. Obstructive sleep apnea and type 2 diabetes: interacting epidemics. Chest. 2008;133(2):496-506.

74. Hamilton GS, Naughton MT. Impact of obstructive sleep apnoea on diabetes and cardiovascular disease. Med J Aust. 2013;199(8):S27-30.

75. Weaver TE, George CFP. Cognition and performance in patients with obstructive sleep apnea. In: Kryger M, Roth T, Dement WC, eds. Principles and Practice of Sleep Medicine. 5th ed. Philadelphia: W. B. Saunders, 2011.

76. Jackson ML, Howard ME, Barnes M. Cognition and daytime functioning in sleep-related breathing disorders. Prog Brain Res. 2011;190:53-68.

77. Omachi TA, Claman DM, Blanc PD, Eisner MD. Obstructive sleep apnea: a risk factor for work disability. Sleep. 2009;32(6):791-798.

78. Leger D, Bayon V, Laaban JP, Philip P. Impact of sleep apnea on economics. Sleep Med Rev. 2012;16(5):455-462.

79. Finn L, Young T, Palta M, Fryback DG. Sleep-disordered breathing and self-reported general health status in the Wisconsin Sleep Cohort Study. Sleep. 1998;21(7):701-706.

80. Baldwin CM, Griffith KA, Nieto FJ, O'Connor GT, Walsleben JA, Redline S. The association of sleepdisordered breathing and sleep symptoms with quality of life in the Sleep Heart Health Study. Sleep. $2001 ; 24(1): 96-105$. 
81. Ellen RL, Marshall SC, Palayew M, Molnar FJ, Wilson KG, Man-Son-Hing M. Systematic review of motor vehicle crash risk in persons with sleep apnea. J Clin Sleep Med. 2006;2(2):193-200.

82. Buchanan P, Grunstein R. Positive airway pressure treatment for obstructive sleep apnea-hypopnea syndrome. In: Kryger M, Roth T, Dement WC, eds. Principles and Practice of Sleep Medicine. 5th ed. Philadelphia, Pennsylvania: W. B. Saunders, 2011.

83. Fava C, Dorigoni S, Dalle Vedove F, et al. Effect of CPAP on blood pressure in patients with OSA/hypopnea: a systematic review and meta-analysis. Chest. 2014;145(4):762-771.

84. Montesi SB, Edwards BA, Malhotra A, Bakker JP. The effect of continuous positive airway pressure treatment on blood pressure: a systematic review and meta-analysis of randomized controlled trials. $\mathrm{J}$ Clin Sleep Med. 2012;8(5):587-596.

85. Marin JM, Carrizo SJ, Vicente E, Agusti AG. Long-term cardiovascular outcomes in men with obstructive sleep apnoea-hypopnoea with or without treatment with continuous positive airway pressure: an observational study. Lancet. 2005;365(9464):1046-1053.

86. McEvoy RD, Antic NA, Heeley E, et al. CPAP for prevention of cardiovascular events in obstructive sleep apnea. N Engl J Med. 2016;375(10):919-931.

87. Ip MS, Tse H-F, Lam B, Tsang KW, Lam W-K. Endothelial function in obstructive sleep apnea and response to treatment. Am J Respir Crit Care Med. 2004;169(3):348-353.

88. Chakhtoura M, Azar ST. Continuous positive airway pressure and type 2 diabetes mellitus. Diabetes Metab Syndr. 2012;6(3):176-179.

89. Avlonitou E, Kapsimalis F, Varouchakis G, Vardavas Cl, Behrakis P. Adherence to CPAP therapy improves quality of life and reduces symptoms among obstructive sleep apnea syndrome patients. Sleep Breath. 2012;16(2):563-569.

90. Tregear S, Reston J, Schoelles K, Phillips B. Continuous positive airway pressure reduces risk of motor vehicle crash among drivers with obstructive sleep apnea: systematic review and meta-analysis. Sleep. 2010;33(10):1373-1380.

91. Kylstra WA, Aaronson JA, Hofman WF, Schmand BA. Neuropsychological functioning after CPAP treatment in obstructive sleep apnea: a meta-analysis. Sleep Med Rev. 2013;17(5):341-347.

92. Izci B, Vennelle M, Liston WA, Dundas KC, Calder AA, Douglas NJ. Sleep-disordered breathing and upper airway size in pregnancy and post-partum. Eur Respir J. 2006;27(2):321-327.

93. McAuliffe F, Kametas N, Costello J, Rafferty GF, Greenough A, Nicolaides K. Respiratory function in singleton and twin pregnancy. BJOG. 2002;109(7):765-769.

94. Weinberger SE, Weiss ST, Cohen WR, Weiss JW, Johnson TS. Pregnancy and the lung. Am Rev Respir Dis. 1980;121(3):559-581.

95. Edwards N, Sullivan CA. Sleep-disordered breathing in pregnancy. Sleep Med Clin. 2008;3(1):81-95.

96. Elkus R, Popovich J. Respiratory physiology in pregnancy. Clin Chest Med. 1992;13(4):555-565.

97. Contreras G, GutiéRrez M, Beroíza T, et al. Ventilatory drive and respiratory muscle function in pregnancy. Am Rev Respir Dis. 1991;144(4):837-841.

98. Popovic RM, White DP. Upper airway muscle activity in normal women: influence of hormonal status. J Appl Physiol. 1998;84(3):1055-1062.

99. Edwards N, Middleton PG, Blyton DM, Sullivan CE. Sleep disordered breathing and pregnancy. Thorax. 2002;57(6):555-558.

100. Wilson DL, Barnes M, Ellett L, Permezel M, Jackson M, Crowe SF. Decreased sleep efficiency, increased wake after sleep onset and increased cortical arousals in late pregnancy. Aust $\mathrm{N} Z \mathrm{~J}$ Obstet Gynaecol. 2011;51(1):38-46.

101. Hertz G, Fast A, Feinsilver SH, Albertario CL, Schulman H, Fein AM. Sleep in normal late pregnancy. Sleep. 1992;15(3):246-251.

102. Loube DI, Poceta JS, Morales MC, Peacock MD, Mitler MM. Self-reported snoring in pregnancy: association with fetal outcome. Chest. 1996;109(4):885-889.

103. Ursavas A, Karadag M, Nalci N, Ercan I, Gozu RO. Self-reported snoring, maternal obesity and neck circumference as risk factors for pregnancy-induced hypertension and preeclampsia. Respiration. 2008;76(1):33-39.

104. O'Brien LM, Bullough AS, Owusu JT, et al. Pregnancy-onset habitual snoring, gestational hypertension, and preeclampsia: prospective cohort study. Am J Obstet Gynecol. 2012;207(6):487.e1-9.

105. Pien GW, Fife D, Pack Al, Nkwuo JE, Schwab RJ. Changes in symptoms of sleep-disordered breathing during pregnancy. Sleep. 2005;28(10):1299-1305.

106. Facco FL, Ouyang DW, Zee PC, Grobman WA. Development of a pregnancy-specific screening tool for sleep apnea. J Clin Sleep Med. 2012;8(4):389-394.

107. Olivarez SA, Maheshwari B, McCarthy M, et al. Prospective trial on obstructive sleep apnea in pregnancy and fetal heart rate monitoring. Obstet Gynecol. 2010;202(6):552.e1-7. 
108. Wilson DL, Fung AM, Walker SP, O'Donoghue F, Howard M, Barnes M. Can we predict sleepdisordered breathing in pregnancy? The clinical utility of symptoms. J Sleep Res. 2013;22(6):670-678.

109. Antony KM, Agrawal A, Arndt ME, et al. Obstructive sleep apnea in pregnancy: reliability of prevalence and prediction estimates. J Perinatol. 2014;34(8):587-593.

110. Netzer NC, Stoohs RA, Netzer CM, Clark K, Strohl KP. Using the Berlin Questionnaire to identify patients at risk for the sleep apnea syndrome. Ann Intern Med. 1999;131(7):485-491.

111. Johns MW. A new method for measuring daytime sleepiness: the Epworth sleepiness scale. Sleep. 1991;14(6):540-545.

112. Maislin G, Pack Al, Kribbs NB, et al. A survey screen for prediction of apnea. Sleep. 1995;18(3):158166.

113. Guilleminault $C$, Stoohs RA, Clerk A, Cetel M, Maistros P. A cause of excessive daytime sleepiness. The upper airway resistance syndrome. Chest. 1993;104(3):781-787.

114. Pepin J, Guillot M, Tamisier R, Lévy P. The upper airway resistance syndrome. Respiration. 2012;83(6):559-566.

115. de Godoy LB, Luz GP, Palombini LO, et al. Upper airway resistance syndrome patients have worse sleep quality compared to mild obstructive sleep apnea. PLoS One. 2016;11(5):e0156244.

116. Guilleminault C, Stoohs R, Shiomi T, Kushida C, Schnittger I. Upper airway resistance syndrome, nocturnal blood pressure monitoring, and borderline hypertension. Chest. 1996;109(4):901-908.

117. Guilleminault C, Black JE, Palombini L, Ohayon M. A clinical investigation of obstructive sleep apnea syndrome (OSAS) and upper airway resistance syndrome (UARS) patients. Sleep Med. 2000;1(1):5156.

118. Stoohs RA, Knaack L, Blum HC, Janicki J, Hohenhorst W. Differences in clinical features of upper airway resistance syndrome, primary snoring, and obstructive sleep apnea/hypopnea syndrome. Sleep Med. 2008;9(2):121-128.

119. Leech JA, Önal E, Dulberg C, Lopata MA. A comparison of men and women with occlusive sleep apnea syndrome. Chest. 1988;94(5):983-988.

120. Guilleminault C, Querra-Salva M, Chowdhuri S, Poyares D. Normal pregnancy, daytime sleeping, snoring and blood pressure. Sleep Med. 2000;1(4):289-297.

121. Connolly G, Razak ARA, Hayanga A, Russell A, McKenna P, McNicholas WT. Inspiratory flow limitation during sleep in pre-eclampsia: comparison with normal pregnant and nonpregnant women. Eur Respir J. 2001;18(4):672-676.

122. Facco FL, Ouyang DW, Zee PC, Grobman WA. Sleep disordered breathing in a high-risk cohort: prevalence and severity across pregnancy. Am J Perinatol. 2014;31(10):899-904 .

123. Facco FL, Parker CB, Reddy UM, et al. Association between sleep-disordered breathing and hypertensive disorders of pregnancy and gestational diabetes mellitus. Obstet Gynecol. 2017;129(1):31-41.

124. Louis J, Auckley D, Miladinovic B, et al. Perinatal outcomes associated with obstructive sleep apnea in obese pregnant women. Obstet Gynecol. 2012;120(5):1085-1092.

125. Edwards N, Blyton DM, Hennessy A, Sullivan CE. Severity of sleep-disordered breathing improves following parturition. Sleep. 2005;28(6):737-741.

126. Chen BY, He QY. A multi-centre study on the association between sleep apnea and prevalence of hypertension. Zhonghua Jie He He Hu Xi Za Zhi [Chinese Journal of Tuberculosis and Respiratory Diseases 2007;30(12):894-897.

127. Pedrosa RP, Barros IML, Drager LF, et al. OSA is common and independently associated with hypertension and increased arterial stiffness in consecutive perimenopausal women. Chest. 2014;146(1):66-72.

128. Kim J, Yi H, Shin KR, Kim JH, Jung KH, Shin C. Snoring as an independent risk factor for hypertension in the nonobese population: the Korean Health and Genome Study. Am J Hypertens. 2007;20(8):819824.

129. Koskenvuo M, Kaprio J, Partinen M, Langinvainio H, Sarna S, Heikkila K. Snoring as a risk factor for hypertension and angina pectoris. Lancet. 1985;1(8434):893-896.

130. Chen JS, Roberts CL, Simpson JM, Ford JB. Prevalence of pre-eclampsia, pregnancy hypertension and gestational diabetes in population-based data: impact of different ascertainment methods on outcomes. Aust N Z J Obstet Gynaecol. 2012;52(1):91-95.

131. Roberts CL, Ford JB, Algert CS, et al. Population-based trends in pregnancy hypertension and preeclampsia: an international comparative study. BMJ Open. 2011;1(1):e000101.

132. Lowe SA, Bowyer L, Lust K, et al. The SOMANZ guidelines for the management of hypertensive disorders of pregnancy 2014. Aust N Z J Obstet Gynaecol. 2015;55(5):e1-e29.

133. Duley L. The global impact of pre-eclampsia and eclampsia. Semin Perinatol. 2009;33(3):130-137. 
134. Tranquilli AL, Dekker G, Magee $\mathrm{L}$, et al. The classification, diagnosis and management of the hypertensive disorders of pregnancy: A revised statement from the ISSHP. Pregnancy Hypertens 2014;4(2):97-104.

135. Zhou Y, Damsky CH, Chiu K, Roberts JM, Fisher SJ. Preeclampsia is associated with abnormal expression of adhesion molecules by invasive cytotrophoblasts. J Clin Invest. 1993;91(3):950-960.

136. Powe CE, Levine RJ, Karumanchi SA. Preeclampsia, a disease of the maternal endothelium. The role of antiangiogenic factors and implications for later cardiovascular disease. Circulation. 2011;123(24):2856-2869.

137. Roberts J, Lain K. Recent insights into the pathogenesis of pre-eclampsia. Placenta. 2002;23(5):359372.

138. Cindrova-Davies T, Yung H-W, Johns J, et al. Oxidative stress, gene expression, and protein changes induced in the human placenta during labor. Am J Pathol. 2007;171(4):1168-1179.

139. Witlin AG, Saade GR, Mattar F, Sibai BM. Risk factors for abruptio placentae and eclampsia: analysis of 445 consecutively managed women with severe preeclampsia and eclampsia. Am J Obstet Gynecol. 1999;180(6 Pt 1):1322-1329.

140. Sibai BM. Chronic hypertension in pregnancy. Obstet Gynecol. 2002;100(2):369-377.

141. Say L, Chou D, Gemmill A, et al. Global causes of maternal death: a WHO systematic analysis. Lancet Glob Health. 2014;2(6):e323-333.

142. Vogel J, Souza J, Mori R, et al. Maternal complications and perinatal mortality: findings of the World Health Organization Multicountry Survey on Maternal and Newborn Health. BJOG. 2014;121(s1):7688.

143. Ferrer RL, Sibai BM, Mulrow CD, Chiquette E, Stevens KR, Cornell J. Management of mild chronic hypertension during pregnancy: a review. Obstet Gynecol. 2000;96(5 Pt 2):849-860.

144. Melamed N, Ray JG, Hladunewich M, Cox B. Gestational hypertension and preeclampsia: are they the same disease? J Obstet Gynaecol Can. 2014;36(7):642-647.

145. Knuist $\mathrm{M}$, Bonsel $\mathrm{G}$, Zondervan $\mathrm{H}$, Treffers $\mathrm{P}$. Intensification of fetal and maternal surveillance in pregnant women with hypertensive disorders. Int J Gynaecol Obstet. 1998;61(2):127-133.

146. Cruz MO, Gao W, Hibbard JU. Obstetrical and perinatal outcomes among women with gestational hypertension, mild preeclampsia, and mild chronic hypertension. Am J Obstet Gynecol. 2011;205(3):260.e1-9.

147. Shen M, Smith GN, Rodger M, White RR, Walker MC, Wen SW. Comparison of risk factors and outcomes of gestational hypertension and pre-eclampsia. PLoS One. 2017;12(4):e0175914.

148. Heard AR, Dekker GA, Chan A, Jacobs DJ, Vreeburg SA, Priest KR. Hypertension during pregnancy in South Australia, part 1: pregnancy outcomes. Aust N Z J Obstet Gynaecol. 2004;44(5):404-409.

149. Sibai BM, Spinnato JA, Watson DL, Hill GA, Anderson GD. Pregnancy outcome in 303 cases with severe preeclampsia. Obstet Gynecol. 1984;64(3):319-325.

150. Hauth JC, Ewell MG, Levine RJ, et al. Pregnancy outcomes in healthy nulliparas who developed hypertension. Calcium for Preeclampsia Prevention Study Group. Obstet Gynecol. 2000;95(1):24-28.

151. Buchbinder R, Sibai BM, Caritis S, et al. Adverse perinatal outcomes are significantly higher in severe gestational hypertension than in mild preeclampsia. Am J Obstet Gynecol. 2002;186(1):66-71.

152. Lisonkova S, Joseph KS. Incidence of preeclampsia: risk factors and outcomes associated with earlyversus late-onset disease. Am J Obstet Gynecol. 2013;209(6):544.e1-12.

153. Lisonkova S, Sabr Y, Mayer C, Young C, Skoll A, Joseph KS. Maternal morbidity associated with earlyonset and late-onset preeclampsia. Obstet Gynecol. 2014;124(4):771-781.

154. Ganzevoort W, Rep A, de Vries JI, Bonsel GJ, Wolf H. Prediction of maternal complications and adverse infant outcome at admission for temporizing management of early-onset severe hypertensive disorders of pregnancy. Am J Obstet Gynecol. 2006;195(2):495-503.

155. Saudan P, Brown MA, Buddle ML, Jones M. Does gestational hypertension become pre-eclampsia? BJOG. 1998;105(11):1177-1184.

156. Lykke JA, Langhoff-Roos J, Sibai BM, Funai EF, Triche EW, Paidas MJ. Hypertensive pregnancy disorders and subsequent cardiovascular morbidity and type 2 diabetes mellitus in the mother. Hypertension. 2009;53(6):944-951.

157. Bellamy L, Casas J-P, Hingorani AD, Williams DJ. Pre-eclampsia and risk of cardiovascular disease and cancer in later life: systematic review and meta-analysis. BMJ. 2007;335(7627):974.

158. Whitehead C, Tong S, Wilson D, Howard M, Walker SP. Treatment of early-onset preeclampsia with continuous positive airway pressure. Obstet Gynecol. 2015;125(5):1106-1109.

159. Maynard SE, Min J-Y, Merchan J, et al. Excess placental soluble fms-like tyrosine kinase 1 (sFlt1) may contribute to endothelial dysfunction, hypertension, and proteinuria in preeclampsia. J Clin Invest. 2003;111(5):649-658.

160. Hubel CA. Oxidative stress in the pathogenesis of preeclampsia. Exp Biol Med. 1999;222(3):222-235. 
161. Somers VK, Javaheri S. Cardiovascular effects of sleep-related breathing disorders. In: Kryger M, Roth T, Dement WC, eds. Principles and Practice of Sleep Medicine. 5th ed. Philadelphia: W. B. Saunders, 2011.

162. Schobel HP, Fischer T, Heuszer K, Geiger H, Schmieder RE. Preeclampsia-a state of sympathetic overactivity. N Engl J Med. 1996;335(20):1480-1485.

163. Lanfranchi PA, Somers VK. Cardiovascular physiology. Autonomic control in health and in sleep disorders. In: Kryger M, Roth T, Dement WC, eds. Principles and Practice of Sleep Medicine. 5th ed. Philadelphia: W. B. Saunders, 2011.

164. Somers VK, Dyken ME, Clary MP, Abboud FM. Sympathetic neural mechanisms in obstructive sleep apnea. J Clin Invest. 1995;96(4):1897-1904.

165. Gilmartin GS, Lynch M, Tamisier R, Weiss JW. Chronic intermittent hypoxia in humans during 28 nights results in blood pressure elevation and increased muscle sympathetic nerve activity. Am J Physiol Heart Circ Physiol. 2010;299(3):H925-H931.

166. Davies R, Belt P, Roberts S, Ali N, Stradling J. Arterial blood pressure responses to graded transient arousal from sleep in normal humans. J Appl Physiol. 1993;74(3):1123-1130.

167. Shimizu T, Takahashi Y, Suzuki K, et al. Muscle nerve sympathetic activity during sleep and its change with arousal response. J Sleep Res. 1992;1(3):178-185.

168. Azarbarzin A, Ostrowski M, Hanly P, Younes M. Relationship between arousal intensity and heart rate response to arousal. Sleep. 2014;37(4):645-653.

169. Blyton DM, Sullivan CE, Edwards N. Reduced nocturnal cardiac output associated with preeclampsia is minimized with the use of nocturnal nasal CPAP. Sleep. 2004;27(1):79-84.

170. Bosio PM, McKenna PJ, Conroy R, O'Herlihy C. Maternal central hemodynamics in hypertensive disorders of pregnancy. Obstet Gynecol. 1999;94(6):978-984.

171. Yang J, Yang Y, Wang K. Central and peripheral hemodynamics in severe preeclampsia. Acta Obstet Gynecol Scand. 1996;75(2):120-126.

172. Prabhakar NR. Oxygen sensing during intermittent hypoxia: cellular and molecular mechanisms. J Appl Physiol. 2001;90(5):1986-1994.

173. Lavie L. Obstructive sleep apnoea syndrome-an oxidative stress disorder. Sleep Med Rev. 2003;7(1):35-51.

174. Lavie L. Sleep-disordered breathing and cerebrovascular disease: a mechanistic approach. Neurol Clin. 2005;23(4):1059-1075.

175. Ryan S, Taylor CT, McNicholas WT. Predictors of elevated nuclear factor-kappa B-dependent genes in obstructive sleep apnea syndrome. Am J Respir Crit Care Med. 2006;174(7):824-830.

176. Nadeem R, Molnar J, Madbouly EM, et al. Serum inflammatory markers in obstructive sleep apnea: a meta-analysis. J Clin Sleep Med. 2013;9(10):1003-1012.

177. Ouyang $\mathrm{Y}$, Li S, Zhang $\mathrm{Q}$, Cai $\mathrm{H}$, Chen $\mathrm{H}$. Interactions between inflammatory and oxidative stress in preeclampsia. Hypertens Pregnancy. 2009;28(1):56-62.

178. Teran E, Escudero C, Moya W, Flores M, Vallance P, Lopez-Jaramillo P. Elevated C-reactive protein and pro-inflammatory cytokines in Andean women with pre-eclampsia. Int J Gynaecol Obstet. $2001 ; 75(3): 243-249$.

179. Xiao JP, Yin YX, Gao YF, et al. The increased maternal serum levels of IL-6 are associated with the severity and onset of preeclampsia. Cytokine. 2012;60(3):856-860.

180. Lohsoonthorn V, Qu C, Williams M. Maternal serum C-reactive protein concentrations in early pregnancy and subsequent risk of preterm delivery. Clin Biochem. 2007;40(5-6):330-335.

181. Huppertz B. Placental origins of preeclampsia: challenging the current hypothesis. Hypertension. 2008;51(4):970-975.

182. Haider S, Knöfler M. Human tumour necrosis factor: physiological and pathological roles in placenta and endometrium. Placenta. 2009;30(2):111-123.

183. Salamonsen LA, Hannan NJ, Dimitriadis E. Cytokines and chemokines during human embryo implantation: roles in implantation and early placentation. Semin Reprod Med. 2007;25(6):437-444.

184. Yinon D, Lowenstein L, Suraya S, et al. Pre-eclampsia is associated with sleep disordered breathing and endothelial dysfunction. Eur Respir J. 2006;27(2):328-333.

185. Benyo DF, Miles TM, Conrad KP. Hypoxia stimulates cytokine production by villous explants from the human placenta. J Clin Endocrinol Metab. 1997;82(5):1582-1588.

186. Adair T, Montani J. Angiogenesis. San Rafael, CA: Morgan \& Claypool Life Sciences, 2010.

187. Maynard SE, Karumanchi SA. Angiogenic factors and preeclampsia. Semin Nephrol. 2011;31(1):3346.

188. Levine RJ, Maynard SE, Qian C, et al. Circulating angiogenic factors and the risk of preeclampsia. N Engl J Med. 2004;350(7):672-683. 
189. Robinson CJ, Johnson DD, Chang EY, Armstrong DM, Wang W. Evaluation of placenta growth factor and soluble fms-like tyrosine kinase 1 receptor levels in mild and severe preeclampsia. Am J Obstet Gynecol. 2006;195(1):255-259.

190. Levine RJ, Lam C, Qian C, et al. Soluble endoglin and other circulating antiangiogenic factors in preeclampsia. N Engl J Med. 2006;355(10):992-1005.

191. Verlohren S, Herraiz I, Lapaire O, et al. The sFlt-1/PIGF ratio in different types of hypertensive pregnancy disorders and its prognostic potential in preeclamptic patients. Am J Obstet Gynecol. 2012;206(1):58.e1-e8.

192. Buhimschi CS, Norwitz ER, Funai E, et al. Urinary angiogenic factors cluster hypertensive disorders and identify women with severe preeclampsia. Am J Obstet Gynecol. 2005;192(3):734-41.

193. Engels T, Pape J, Schoofs K, Henrich W, Verlohren S. Automated measurement of sFlt1, PIGF and sFlt1/PIGF ratio in differential diagnosis of hypertensive pregnancy disorders. Hypertens Pregnancy. 2013;32(4):459-73.

194. Lim JH, Kim SY, Park SY, Yang JH, Kim MY, Ryu HM. Effective prediction of preeclampsia by a combined ratio of angiogenesis-related factors. Obstet Gynecol. 2008;111(6):1403-9.

195. Redman C, Sargent I. Placental stress and pre-eclampsia: a revised view. Placenta. 2009;30 Suppl A:S38-42.

196. Mohsenin V, Urbano F. Circulating antiangiogenic proteins in obstructive sleep apnea and hypertension. Respir Med. 2011;105(5):801-807.

197. Jafari B, Mohsenin V. Endothelial dysfunction and hypertension in obstructive sleep apnea-Is it due to intermittent hypoxia? J Cardiovasc Dis Res. 2013;4(2):87-91.

198. Bourjeily G, Curran P, Butterfield K, Maredia H, Carpenter M, Lambert-Messerlian G. Placenta-secreted circulating markers in pregnant women with obstructive sleep apnea. J Perinat Med. 2015;43(1):81-7.

199. Xiong X, Mayes D, Demianczuk N, et al. Impact of pregnancy-induced hypertension on fetal growth. Am J Obstet Gynecol. 1999;180(1):207-213.

200. Nardozza LMM, Caetano ACR, Zamarian ACP, et al. Fetal growth restriction: current knowledge. Arch Gynecol Obstet. 2017;295(5):1061-1077.

201. Julian CG, Yang IV, Browne VA, et al. Inhibition of peroxisome proliferator-activated receptor Y: a potential link between chronic maternal hypoxia and impaired fetal growth. FASEB J. 2014;28(3):12681279.

202. Moore LG, Charles SM, Julian CG. Humans at high altitude: hypoxia and fetal growth. Respir Physiol Neurobiol. 2011;178(1):181-190.

203. Lueder FL, Kim S-B, Buroker CA, Bangalore SA, Ogata ES. Chronic maternal hypoxia retards fetal growth and increases glucose utilization of select fetal tissues in the rat. Metabolism. 1995;44(4):532537.

204. Kovo M, Schreiber L, Ben-Haroush A, et al. The placental factor in early-and late-onset normotensive fetal growth restriction. Placenta. 2013;34(4):320-324.

205. Umbers AJ, Boeuf $P$, Clapham C, et al. Placental malaria-associated inflammation disturbs the insulinlike growth factor axis of fetal growth regulation. J Infect Dis. 2011;203(4):561-569.

206. Tauman R, Many A, Deutsch V, et al. Maternal snoring during pregnancy is associated with enhanced fetal erythropoiesis - a preliminary study. Sleep Med. 2011;12(5):518-522.

207. Jones JI, Clemmons DR. Insulin-like growth factors and their binding proteins: biological actions. Endocr Rev. 1995;16(1):3-34.

208. Forbes K, Westwood M. The IGF axis and placental function. A mini review. Horm Res. 2008;69(3):129137.

209. Klauwer D, Blum W, Hanitsch S, Rascher W, Lee P, Kiess W. IGF-I, IGF-II, free IGF-I and IGFBP-1,-2 and-3 levels in venous cord blood: relationship to birthweight, length and gestational age in healthy newborns. Acta Paediatr. 1997;86(8):826-833.

210. Ong K, Kratzsch J, Kiess W, Costello M, Scott C, Dunger D. Size at birth and cord blood levels of insulin, insulin-like growth factor I (IGF-I), IGF-II, IGF-binding protein-1 (IGFBP-1), IGFBP-3, and the soluble IGF-II/mannose-6-phosphate receptor in term human infants. J Clin Endocrinol Metab. 2000;85(11):4266-4269.

211. Nawathe AR, Christian M, Kim SH, Johnson M, Savvidou MD, Terzidou V. Insulin-like growth factor axis in pregnancies affected by fetal growth disorders. Clin Epigenetics. 2016;8:11.

212. Watson CS, Bialek P, Anzo M, Khosravi J, Yee S-P, Han VK. Elevated circulating insulin-like growth factor binding protein-1 is sufficient to cause fetal growth restriction. Endocrinology. 2006;147(3):11751186.

213. Street ME, Seghini P, Fieni S, et al. Changes in interleukin-6 and IGF system and their relationships in placenta and cord blood in newborns with fetal growth restriction compared with controls. Eur $\mathrm{J}$ Endocrinol. 2006;155(4):567-574. 
214. Ursavas A, Karadag M, Ilcol YO, et al. Low level of IGF-1 in obesity may be related to obstructive sleep apnea syndrome. Lung. 2007;185(5):309-314.

215. Makino S, Fujiwara M, Handa $\mathrm{H}$, et al. Plasma dehydroepiandrosterone sulphate and insulin-like growth factor I levels in obstructive sleep apnoea syndrome. Clin Endocrinol (Oxf.). 2012;76(4):593-601.

216. Mysliwiec V, Gill J, Matsangas P, Baxter T, Barr T, Roth BJ. IGF-1: a potential biomarker for efficacy of sleep improvement with automatic airway pressure therapy for obstructive sleep apnea? Sleep Breath. 2015;19(4):1221-1228.

217. Briana DD, Malamitsi-Puchner A. Intrauterine growth restriction and adult disease: the role of adipocytokines. Eur J Endocrinol. 2009;160(3):337-347.

218. Amarilyo G, Oren A, Mimouni F, Ochshorn Y, Deutsch V, Mandel D. Increased cord serum inflammatory markers in small-for-gestational-age neonates. J Perinatol. 2011;31(1):30-32.

219. Seremak-Mrozikiewicz A, Dubiel M, Drews K, Gudmundsson S, Mrozikiewicz PM. TNF-alpha gene polymorphism and fetal doppler velocimetry in intrauterine growth restriction. Neuro Endocrinol Lett. 2008;29(4):493-499.

220. Karowicz-Bilinska A, Kedziora-Kornatowska K, Bartosz G. Indices of oxidative stress in pregnancy with fetal growth restriction. Free Radic Res. 2007;41(8):870-873.

221. Bourjeily G, Butterfield K, Curran P, Lambert-Messerlian G. Obstructive sleep apnea is associated with alterations in markers of fetoplacental wellbeing. J Matern Fetal Neonatal Med. 2015;28(3):262-266.

222. Manning FA. General principles and applications of ultrasonography. In: Creasy RK, Resnik R, lams JD, eds. Maternal-Fetal Medicine: Principles and Practice. 5th ed. Philadelphia, PA: W. B. Saunders, 2004:169-206.

223. Hadlock FP, Harrist R, Sharman RS, Deter RL, Park SK. Estimation of fetal weight with the use of head, body, and femur measurements-a prospective study. Am J Obstet Gynecol. 1985;151(3):333-337.

224. Gardosi J, Mongelli M, Wilcox M, Chang A. An adjustable fetal weight standard. Ultrasound Obstet Gynecol. 1995;6(3):168-174.

225. McCowan LM, Harding JE, Stewart AW. Customised birthweight centiles predict SGA pregnancies with perinatal morbidity. BJOG. 2005;112(8):1026-1033.

226. Jong CL, Gardosi J, Dekker GA, Colenbrander GJ, Geijn HP. Application of a customised birthweight standard in the assessment of perinatal outcome in a high risk population. BJOG. 1998;105(5):531535.

227. Clausson B, Gardosi J, Francis A, Cnattingius S. Perinatal outcome in SGA births defined by customised versus population-based birthweight standards. BJOG. 2001;108(8):830-834.

228. Hui L, Challis D. Diagnosis and management of fetal growth restriction: the role of fetal therapy. Best Pract Res Clin Obstet Gynaecol. 2008;22(1):139-158.

229. Reeves S, Galan HL. Fetal growth restriction. In: Berghella V, ed. Maternal-Fetal Evidence Based Guidelines. 2nd ed. London: Informa Healthcare, 2012.

230. Fang S. Management of preterm infants with intrauterine growth restriction. Early Hum Dev. 2005;81(11):889-900.

231. Levy-Marchal $C$, Jaquet $D$. Long-term metabolic consequences of being born small for gestational age. Pediatr Diabetes 2004;5(3):147-153.

232. Ott WJ. The diagnosis of altered fetal growth. Obstet Gynecol Clin North Am. 1988;15(2):237-263.

233. Ounsted M, Moar V, Scott A. Risk factors associated with small-for-dates and large-for-dates infants. BJOG. 1985;92(3):226-232.

234. Ravishankar S, Bourjeily G, Lambert-Messerlian G, He M, De Paepe ME, Gündoğan F. Evidence of placental hypoxia in maternal sleep disordered breathing. Pediatr Dev Pathol. 2015;18(5):380-386.

235. Bobrow CS, Soothill PW. Causes and consequences of fetal acidosis. Arch Dis Child Fetal Neonatal Ed. 1999;80(3):F246-249.

236. Izci-Balserak B, Pien GW. Sleep-disordered breathing and pregnancy: Potential mechanisms and evidence for maternal and fetal morbidity. Curr Opin Pulm Med. 2010;16(6):574-582.

237. Soothill PW, Ajayi RA, Campbell S, Ross EM, Nicolaides KH. Fetal oxygenation at cordocentesis, maternal smoking and childhood neuro-development. Eur J Obstet Gynecol Reprod Biol. 1995;59(1):21-24.

238. Schwartz JE, Kovach A, Meyer J, McConnell C, Iwamoto HS. Brief, intermittent hypoxia restricts fetal growth in Sprague-Dawley rats. Biol Neonate. 1997;73(5):313-319.

239. Gozal D, Reeves SR, Row BW, Neville JJ, Guo SZ, Lipton AJ. Respiratory effects of gestational intermittent hypoxia in the developing rat. Am J Respir Crit Care Med. 2003;167(11):1540-1547.

240. Sahin FK, Koken G, Cosar E, et al. Obstructive sleep apnea in pregnancy and fetal outcome. Int J Gynaecol Obstet. 2008;100(2):141-146.

241. Roush SF, Bell L. Obstructive sleep apnea in pregnancy. J Am Board Fam Pract. 2004;17(4):292-294. 
242. Fung AM, Wilson DL, Lappas $M$, et al. Effects of maternal obstructive sleep apnoea on fetal growth: a prospective cohort study. PLoS One. 2013;8(7):e68057.

243. Hermansen M. Nucleated red blood cells in the fetus and newborn. Arch Dis Child Fetal Neonatal Ed. $2001 ; 84(3): 211-215$.

244. Parer JT, Nageotte MP. Intrapartum fetal surveillance. In: Creasy RK, Resnik R, lams JD, eds. Maternal-Fetal Medicine: Principles and Practice. 5th ed. Philadelphia, PA: W. B. Saunders, 2004:403427.

245. Grivell RM, Alfirevic Z, Gyte GML, Devane D. Antenatal cardiotocography for fetal assessment. Cochrane Database Syst. Rev. 2015;(9):CD007863.

246. Alfirevic Z, Devane D, Gyte GML, Cuthbert A. Continuous cardiotocography (CTG) as a form of electronic fetal monitoring (EFM) for fetal assessment during labour. Cochrane Database Syst. Rev. 2017;(2):CD006066.

247. The Royal Australian and New Zealand College of Obstetricians and Gynaecologists. The normal CTG. 2017 [cited 2017 December 12]; Available from: https://ofsep.fsep.edu.au/mod/book/view.php?id=409

248. The Royal Australian and New Zealand College of Obstetricians and Gynaecologists. The abnormal CTG. 2017 [cited 2017 December 12]; Available from: https://ofsep.fsep.edu.au/mod/book/view.php?id=411

249. Roberts JM. Pregnancy-related hypertension. In: Creasy RK, Resnik R, lams JD, eds. Maternal-Fetal Medicine: Principles and Practice. Philadelphia, PA: W. B. Saunders, 2004:859-899.

250. Furukawa $\mathrm{S}$, Sameshima $\mathrm{H}$, Ikenoue $\mathrm{T}$. Intrapartum late deceleration develops more frequently in preeclamptic women with severe proteinuria. J Obstet Gynaecol Res. 2006;32(1):68-73.

251. Court D, Parer J. Experimental studies of fetal asphyxia and fetal heart rate interpretation. In: Nathanielsz P, Parer J, eds. Research in Perinatal Medicine, vol I. New York: Perinatology Press, 1984.

252. Swansburg M, Brown C, Hains S, Smith G, Kisilevsky B. Maternal cardiac autonomic function and fetal heart rate in preeclamptic compared to normotensive pregnancies. Can $\mathrm{J}$ Cardiovasc Nurs. 2005;15(3):42-52.

253. Higgins N, Leong E, Park CS, Facco FL, McCarthy RJ, Wong CA. The Berlin Questionnaire for assessment of sleep disordered breathing risk in parturients and non-pregnant women. Int $\mathrm{J}$ Obstet Anesth. 2011;20(1):22-25.

254. Olivarez SA, Ferres M, Antony K, et al. Obstructive sleep apnea screening in pregnancy, perinatal outcomes, and impact of maternal obesity. Am J Perinatol. 2011;28(8):651-658.

255. Ko HS, Kim MY, Kim YH, et al. Obstructive sleep apnea screening and perinatal outcomes in Korean pregnant women. Arch Gynecol Obstet. 2013;287(3):429-433.

256. Sarberg M, Svanborg E, Wirehn A, Josefsson A. Snoring during pregnancy and its relation to sleepiness and pregnancy outcome - a prospective study. BMC Pregnancy Childbirth. 2014;14:15.

257. Champagne K, Schwartzman K, Opatrny L, et al. Obstructive sleep apnoea and its association with gestational hypertension. Eur Respir J. 2009;33(3):559-565.

258. Reid J, Glew RA, Skomro R, et al. Sleep disordered breathing and gestational hypertension: postpartum follow-up study. Sleep. 2013;36(5):717-721.

259. Facco FL, Lappen J, Lim C, Zee PC, Grobman WA. Preeclampsia and sleep-disordered breathing: A case-control study. Pregnancy Hypertens. 2013;3(2):133-139.

260. O'Brien LM, Bullough A, Chames M, et al. Hypertension, snoring, and obstructive sleep apnoea during pregnancy: a cohort study. BJOG. 2014;121(13):1685-1693.

261. Yin TT, Williams N, Burton C, et al. Hypertension, fetal growth restriction and obstructive sleep apnoea in pregnancy. Eur J Obstet Gynecol Reprod Biol 2008;141(1):35-38.

262. Louis JM, Auckley D, Sokol RJ, Mercer BM. Maternal and neonatal morbidities associated with obstructive sleep apnea complicating pregnancy. Obstet Gynecol. 2010;202(3):261.e1-5.

263. Facco FL, Ouyang DW, Zee PC, et al. Implications of sleep-disordered breathing in pregnancy. Am J Obstet Gynecol. 2014;210(6):559.e1-6.

264. Young T, Peppard PE, Taheri S. Excess weight and sleep-disordered breathing. J Appl Physiol. 2005;99(4):1592-1599.

265. Senaratna CV, Perret JL, Lodge CJ, et al. Prevalence of obstructive sleep apnea in the general population: a systematic review. Sleep Med Rev. 2017;34:70-81.

266. Gaillard R, Steegers EA, Hofman A, Jaddoe VW. Associations of maternal obesity with blood pressure and the risks of gestational hypertensive disorders. The Generation R Study. J Hypertens. 2011;29(5):937-944.

267. Catov JM, Ness RB, Kip KE, Olsen J. Risk of early or severe pre-eclampsia related to pre-existing conditions. Int J Epidemiol. 2007;36(2):412-419. 
268. Sullivan C, Zieschang J, Johnson P, Middleton S, Norman M, Clark A. The increase in snoring during pregnancy is associated with an increase in systemic blood pressure. J Sleep Res. 2011;20(Suppl. 1):23.

269. Edwards N, Blyton DM, Kirjavainen TT, Sullivan CE. Hemodynamic responses to obstructive respiratory events during sleep are augmented in women with preeclampsia. Am J Hypertens. 2001;14(11 Pt 1):1090-1095.

270. Edwards N, Blyton DM, Kirjavainen T, Kesby GJ, Sullivan CE. Nasal continuous positive airway pressure reduces sleep-induced blood pressure increments in preeclampsia. Am J Respir Crit Care Med. 2000;162(1):252-257.

271. Guilleminault C, Kreutzer M, Chang JL. Pregnancy, sleep disordered breathing and treatment with nasal continuous positive airway pressure. Sleep Med. 2004;5(1):43-51.

272. Guilleminault C, Palombini L, Poyares D, Takaoka S, Huynh N, El-Sayed Y. Pre-eclampsia and nasal CPAP: Part 1. Early intervention with nasal CPAP in pregnant women with risk-factors for preeclampsia: Preliminary findings. Sleep Med. 2007;9(1):9-14.

273. Poyares D, Guilleminault C, Hachul H, et al. Pre-eclampsia and nasal CPAP: Part 2. Hypertension during pregnancy, chronic snoring, and early nasal CPAP intervention. Sleep Med. 2007;9(1):15-21.

274. Reid J, Taylor-Gjevre R, Gjevre J, et al. Can gestational hypertension be modified by treating nocturnal airflow limitation? J Clin Sleep Med. 2013;9(4):311-317.

275. Qiu C, Enquobahrie D, Frederick IO, Abetew D, Williams MA. Glucose intolerance and gestational diabetes risk in relation to sleep duration and snoring during pregnancy: a pilot study. BMC Womens Health. 2010;10:17.

276. Reutrakul S, Zaidi N, Wroblewski K, et al. Sleep disturbances and their relationship to glucose tolerance in pregnancy. Diabetes Care. 2011;34(11):2454-2457.

277. Izci Balserak B, Jackson N, Ratcliffe SA, Pack AI, Pien GW. Sleep-disordered breathing and daytime napping are associated with maternal hyperglycemia. Sleep Breath. 2013;17(3):1093-1102.

278. Bisson M, Series F, Giguere Y, et al. Gestational diabetes mellitus and sleep-disordered breathing. Obstet Gynecol. 2014;123(3):634-641.

279. O'Brien LM, Bullough AS, Owusu JT, et al. Snoring during pregnancy and delivery outcomes: A cohort study. Sleep. 2013;36(11):1625-1632.

280. Chen YH, Kang JH, Lin CC, Wang IT, Keller JJ, Lin HC. Obstructive sleep apnea and the risk of adverse pregnancy outcomes. Am J Obstet Gynecol. 2012;206(2):136.e1-5.

281. Micheli K, Komninos I, Bagkeris E, et al. Sleep patterns in late pregnancy and risk of preterm birth and fetal growth restriction. Epidemiology. 2011;22(5):738-744.

282. Tauman R, Sivan Y, Katsav S, Greenfeld M, Many A. Maternal snoring during pregnancy is not associated with fetal growth restriction. J Matern Fetal Neonatal Med. 2012;25(8):1283-1286.

283. Stacey T, Thompson JMD, Mitchell EA, Ekeroma AJ, Zuccollo JM, McCowan LME. Assocation between maternal sleep practices and risk of late stillbirth: a case-control study. BMJ. 2011;342:d3403.

284. Blyton D, Skilton MR, Edwards N, Hennessy A, Celermajer DS, Sullivan C. Treatment of sleep disordered breathing reverses low fetal activity levels in preeclampsia. Sleep. 2013;36(1):15-21.

285. Brennecke S. Australian action on pre-eclampsia. 2011 [cited; Available from: www.aapec.org.au]

286. Auger N, Luo Z-C, Nuyt AM, et al. Secular trends in preeclampsia incidence and outcomes in a large Canada database: a longitudinal study over 24 years. Can J Cardiol. 2016;32(8):987.e15-23.

287. Wallis AB, Saftlas AF, Hsia J, Atrash HK. Secular trends in the rates of preeclampsia, eclampsia, and gestational hypertension, United States, 1987-2004. Am J Hypertens. 2008;21(5):521-526.

288. Conde-Agudelo A, Villar J, Lindheimer M. World Health Organization systematic review of screening tests for preeclampsia. Obstet Gynecol. 2004;104(6):1367-1391.

289. Rodriguez A, Tuuli MG, Odibo AO. First-, second-, and third-trimester screening for preeclampsia and intrauterine growth restriction. Clin Lab Med. 2016;36(2):331-351.

290. Worsnop CJ, Naughton MT, Barter CE, Morgan TO, Anderson Al, Pierce RJ. The prevalence of obstructive sleep apnea in hypertensives. Am J Respir Crit Care Med. 1998;157(1):111-115.

291. Bazzano LA, Khan Z, Reynolds K, He J. Effect of nocturnal nasal continuous positive airway pressure on blood pressure in obstructive sleep apnea. Hypertension. 2007;50(2):417-423.

292. Bruyneel M, Ninane V. Unattended home-based polysomnography for sleep disordered breathing: current concepts and perspectives. Sleep Med Rev. 2014;18(4):341-347.

293. Campbell AJ, Neill AM. Home set-up polysomnography in the assessment of suspected obstructive sleep apnea. J Sleep Res. 2011;20(1 Pt 2):207-213.

294. Mykytyn I, Sajkob D, Neill A, McEvoy R. Portable computerized polysomnography in attended and unattended settings. Chest. 1999;115(1):114-122. 
295. Berry RB, Brooks R, Gamaldo CE, et al. The AASM Manual for the Scoring of Sleep and Associated Events: Rules, Terminology and Technical Specifications, Version 2.3. Darien, Illinois: American Academy of Sleep Medicine, 2016.

296. Allen RP, Picchietti D, Hening WA, Trenkwalder C, Walters AS, Montplaisi J. Restless legs syndrome: diagnostic criteria, special considerations, and epidemiology: a report from the restless legs syndrome diagnosis and epidemiology workshop at the National Institutes of Health. Sleep Med. 2003;4(2):101119.

297. Nankervis A, McIntyre HD, Moses R, et al. ADIPS consensus guidelines for the testing and diagnosis of hyperglycaemia in pregnancy in Australia and New Zealand. 2014.

298. Tranquilli AL, Brown MA, Zeeman GG, Dekker G, Sibai BM. The definition of severe and early-onset preeclampsia. Statements from the International Society for the Study of Hypertension in Pregnancy (ISSHP). Pregnancy Hypertens. 2013;3(1):44-47.

299. Gardosi J, Francis A. Customised Weight Centile Calculator - GROW-Centile v6.6. 2013 [Available from: www.gestation.net.]

300. De Jong C, Francis A, Van Geijn H, Gardosi J. Fetal growth rate and adverse perinatal events. Ultrasound Obstet Gynecol. 1999;13(2):86-89.

301. Royal College of Obstetricians and Gynaecologists. The Investigation and Management of the Smallfor-Gestational-Age Fetus. Guideline No. 31. London: RCOG, 2002.

302. Bourjeily G, Ankner G, Mohsenin V. Sleep-disordered breathing in pregnancy. Clin Chest Med. 2011;32(1):175-189.

303. Trojner Bregar A, Tul N, Fabjan Vodušek V, et al. A dose-response relation exists between different classes of pre-gravid obesity and selected perinatal outcomes. Arch Gynecol Obstet. 2017;296(3):465468.

304. Young T, Shahar E, Nieto FJ, et al. Predictors of sleep-disordered breathing in community-dwelling adults: the Sleep Heart Health Study. Arch Intern Med. 2002;162(8):893-900.

305. Strobel RJ, Rosen RC. Obesity and weight loss in obstructive sleep apnea: a critical review. Sleep. 1996;19(2):104-115.

306. Izci B, Riha RL, Martin SE, et al. The upper airway in pregnancy and pre-eclampsia. Am J Respir Crit Care Med. 2003;167(2):137-140.

307. Wilson DL, Walker SP, Fung AM, O'Donoghue F, Barnes M, Howard M. Can we predict sleepdisordered breathing in pregnancy? The clinical utility of symptoms. J Sleep Res. 2013;22(6):670-678.

308. Carlson JT, Hedner J, Elam M, Ejnell H, Sellgren J, Wallin BG. Augmented resting sympathetic activity in awake patients with obstructive sleep apnea. Chest. 1993;103(6):1763-1768.

309. Narkiewicz K, Pesek CA, Kato M, Phillips BG, Davison DE, Somers VK. Baroreflex control of sympathetic nerve activity and heart rate in obstructive sleep apnea. Hypertension. 1998;32(6):10391043.

310. Narkiewicz K, Van De Borne PJ, Pesek CA, Dyken ME, Montano N, Somers VK. Selective potentiation of peripheral chemoreflex sensitivity in obstructive sleep apnea. Circulation. 1999;99(9):1183-1189.

311. Kanagy NL, Walker BR, Nelin LD. Role of endothelin in intermittent hypoxia-induced hypertension. Hypertension. 2001;37(2):511-515.

312. Wang Z, Li A-Y, Guo Q-H, et al. Effects of cyclic intermittent hypoxia on ET-1 responsiveness and endothelial dysfunction of pulmonary arteries in rats. PLoS One. 2013;8(3):e58078.

313. Phillips BG, Narkiewicz K, Pesek CA, Haynes WG, Dyken ME, Somers VK. Effects of obstructive sleep apnea on endothelin-1 and blood pressure. J Hypertens. 1999;17(1):61-66.

314. Saarelainen S, Seppälä E, Laasonen K, Hasan J. Circulating endothelin-1 in obstructive sleep apnea. Endothelium. 1997;5(2):115-118.

315. Baksu B, Davas I, Baksu A, Akyol A, Gulbaba G. Plasma nitric oxide, endothelin-1 and urinary nitric oxide and cyclic guanosine monophosphate levels in hypertensive pregnant women. Int J Gynaecol Obstet. 2005;90(2):112-117.

316. Nishikawa S, Miyamoto A, Yamamoto H, Ohshika H, Kudo R. The relationship between serum nitrate and endothelin-1 concentrations in preeclampsia. Life Sci. 2000;67(12):1447-1454.

317. Taylor RN, Varma M, Teng NN, Roberts JM. Women with preeclampsia have higher plasma endothelin levels than women with normal pregnancies. J Clin Endocrinol Metab. 1990;71(6):1675-1677.

318. Villar J, Carroli G, Wojdyla D, et al. Preeclampsia, gestational hypertension and intrauterine growth restriction, related or independent conditions? Am J Obstet Gynecol. 2006;194(4):921-931.

319. Maloney KF, Heller D, Baergen RN. Types of maternal hypertensive disease and their association with pathologic lesions and clinical factors. Fetal Pediatr Pathol. 2012;31(5):319-323.

320. González-Quintero VH, Smarkusky LP, Jiménez JJ, et al. Elevated plasma endothelial microparticles: preeclampsia versus gestational hypertension. Am J Obstet Gynecol. 2004;191(4):1418-1424. 
321. Noori M, Donald AE, Angelakopoulou A, Hingorani AD, Williams DJ. Prospective study of placental angiogenic factors and maternal vascular function before and after preeclampsia and gestational hypertension. Circulation. 2010;122(5):478-487.

322. Silver HM, Seebeck M, Carlson R. Comparison of total blood volume in normal, preeclamptic, and nonproteinuric gestational hypertensive pregnancy by simultaneous measurement of red blood cell and plasma volumes. Am J Obstet Gynecol. 1998;179(1):87-93.

323. Sibai BM. Diagnosis and management of gestational hypertension and preeclampsia. Obstet Gynecol. 2003;102(1):181-192.

324. George EM, Granger JP. Endothelin: Key mediator of hypertension in preeclampsia. Am J Hypertens. $2011 ; 24(9): 964-969$.

325. Lefkou E, Hunt B. Pre-eclampsia. In: Pavord S, Hunt B, eds. The Obstetric Hematology Manual. Cambridge: Cambridge University Press, 2010:203-217.

326. Zhou Y, Damsky CH, Chiu K, Roberts JM, Fisher SJ. Preeclampsia is associated with abnormal expression of adhesion molecules by invasive cytotrophoblasts. J Clin Invest. 1993;91(3):950-960.

327. Genbacev O, Joslin R, Damsky CH, Polliotti BM, Fisher SJ. Hypoxia alters early gestation human cytotrophoblast differentiation/invasion in vitro and models the placental defects that occur in preeclampsia. J Clin Invest. 1996;97(2):540-550.

328. Eisele H-J, Markart P, Schulz R. Obstructive sleep apnea, oxidative stress, and cardiovascular disease: evidence from human studies. Oxid Med Cell Longev. 2015;2015:608438.

329. Faulx MD, Larkin EK, Hoit BD, Aylor JE, Wright AT, Redline S. Sex influences endothelial function in sleep-disordered breathing. Sleep. 2004;27(6):1113-1120.

330. Dekker G, Sibai B. Primary, secondary, and tertiary prevention of pre-eclampsia. Lancet. 2001;357(9251):209-215.

331. Dekker G, Robillard P-Y. The birth interval hypothesis-does it really indicate the end of the primipaternity hypothesis. J Reprod Immunol. 2003;59(2):245-251.

332. Einarsson JI, Sangi-Haghpeykar H, Gardner MO. Sperm exposure and development of preeclampsia. Am J Obstet Gynecol. 2003;188(5):1241-1243.

333. Deen ME, Ruurda LGC, Wang J, Dekker GA. Risk factors for preeclampsia in multiparous women: primipaternity versus the birth interval hypothesis. J Matern Fetal Neonatal Med. 2006;19(2):79-84.

334. Wang JX, Knottnerus A-M, Schuit G, Norman RJ, Chan A, Dekker GA. Surgically obtained sperm, and risk of gestational hypertension and pre-eclampsia. Lancet. 2002;359(9307):673-674.

335. Dekker GA, Robillard P-Y, Hulsey TC. Immune maladaptation in the etiology of preeclampsia: a review of corroborative epidemiologic studies. Obstet Gynecol Surv. 1998;53(6):377-382.

336. Keck PJ, Hauser SD, Krivi G, et al. Vascular permeability factor, an endothelial cell mitogen related to PDGF. Science. 1989;246(4935):1309-1312.

337. Leung DW, Cachianes G, Kuang W-J, Goeddel DV, Ferrara N. Vascular endothelial growth factor is a secreted angiogenic mitogen. Science. 1989;246(4935):1306-1309.

338. Hertig A, Berkane N, Lefevre G, et al. Maternal serum sFlt1 concentration is an early and reliable predictive marker of preeclampsia. Clin Chem. 2004;50(9):1702-1703.

339. Gilbert JS, Babcock SA, Granger JP. Hypertension produced by reduced uterine perfusion in pregnant rats is associated with increased soluble fms-like tyrosine kinase-1 expression. Hypertension. 2007;50(6):1142-1147.

340. Makris A, Thornton C, Thompson J, et al. Uteroplacental ischemia results in proteinuric hypertension and elevated sFIt-1. Kidney Int. 2007;71(10):977-984.

341. Carmeliet $P$, Moons L, Luttun A, et al. Synergism between vascular endothelial growth factor and placental growth factor contributes to angiogenesis and plasma extravasation in pathological conditions. Nat Med. 2001;7(5):575-583.

342. Levine RJ, Thadhani R, Qian C, et al. Urinary placental growth factor and risk of preeclampsia. JAMA. 2005;293(1):77-85.

343. Barcelo A, Bauça JM, Yañez A, et al. Impact of obstructive sleep apnea on the levels of placental growth factor (PLGF) and their value for predicting short-term adverse outcomes in patients with acute coronary syndrome. PLoS One. 2016;11(3):e0147686.

344. Zamudio S, Borges M, Echalar L, et al. Maternal and fetoplacental hypoxia do not alter circulating angiogenic growth effectors during human pregnancy. Biol Reprod. 2014;90(2):42.

345. Gilbert JS, Ryan MJ, LaMarca BB, Sedeek M, Murphy SR, Granger JP. Pathophysiology of hypertension during preeclampsia: linking placental ischemia with endothelial dysfunction. Am J Physiol Heart Circ Physiol. 2008;294(2):H541-550.

346. Grimpen F, Kanne P, Schulz E, Hagenah G, Hasenfuß G, Andreas S. Endothelin-1 plasma levels are not elevated in patients with obstructive sleep apnoea. Eur Respir J. 2000;15(2):320-325. 
347. Saarelainen S, Hasan J. Circulating endothelin-1 and obstructive sleep apnoea. Eur Respir J. 2000;16(4):794-795.

348. Daly A, Robertson A, Johnson P, et al. PP162. sFlt-1 controlled by CPAP in a pregnant patient with chronic hypertension. Pregnancy Hypertens. 2012;2(3):327.

349. Daly A, Robertson A, Bobek G, Middleton S, Sullivan C, Hennessy A. Sleep disordered breathing controlled by CPAP and sFlt-1 in a pregnant patient with chronic hypertension: Case report and literature review. Obstet Med. 2018;11(1):32-4.

350. Okun ML, Hall M, Coussons-Read ME. Sleep disturbances increase interleukin- 6 production during pregnancy: Implications for pregnancy complications. Reprod Sci. 2007;14(6):560-567.

351. Macdonald-Wallis C, Lawlor DA, Fraser A, May M, Nelson SM, Tilling K. Blood pressure change in normotensive, gestational hypertensive, preeclamptic, and essential hypertensive pregnancies. Hypertension. 2012;59(6):1241-1248.

352. Gaillard R, Bakker R, Willemsen SP, Hofman A, Steegers EA, Jaddoe VW. Blood pressure tracking during pregnancy and the risk of gestational hypertensive disorders: the Generation R Study. Eur Heart J. 2011;32(24):3088-3097.

353. Oparil S, Zaman MA, Calhoun DA. Pathogenesis of hypertension. Ann Intern Med. 2003;139(9):761776.

354. Somers VK, Dyken ME, Clary MP, Abboud FM. Sympathetic neural mechanisms in obstructive sleep apnea. J Clin Invest. 1995;96(4):1897-1904.

355. Imadojemu VA, Mawji Z, Kunselman A, Gray KS, Hogeman CS, Leuenberger UA. Sympathetic chemoreflex responses in obstructive sleep apnea and effects of continuous positive airway pressure therapy. Chest. 2007;131(5):1406-1413.

356. Chapleau MW, Hajduczok G, Abboud FM. Mechanisms of resetting of arterial baroreceptors: an overview. Am J Med Sci. 1988;295(4):327-334.

357. Monahan KD, Leuenberger UA, Ray CA. Effect of repetitive hypoxic apnoeas on baroreflex function in humans. J Physiol. 2006;574(2):605-613.

358. Carlson JT, Hedner JA, Sellgren J, Elam M, Wallin BG. Depressed baroreflex sensitivity in patients with obstructive sleep apnea. Am J Respir Crit Care Med. 1996;154(5):1490-1496.

359. Rouwet E, Tintu A, Schellings M, et al. Hypoxia induces aortic hypertrophic growth, left ventricular dysfunction, and sympathetic hyperinnervation of peripheral arteries in the chick embryo. Circulation. 2002;105(23):2791-2796.

360. Phillips SA, Olson E, Lombard JH, Morgan BJ. Chronic intermittent hypoxia alters NE reactivity and mechanics of skeletal muscle resistance arteries. J Appl Physiol. 2006;100(4):1117-1123.

361. Phillips SA, Olson E, Morgan BJ, Lombard JH. Chronic intermittent hypoxia impairs endotheliumdependent dilation in rat cerebral and skeletal muscle resistance arteries. Am J Physiol Heart Circ Physiol. 2004;286(1):H388-393.

362. Grote L, Hedner J, Peter JH. Mean blood pressure, pulse pressure and grade of hypertension in untreated hypertensive patients with sleep-related breathing disorder. J Hypertens. 2001;19(4):683690.

363. Carlson JT, Hedner JA, Ejnell H, Peterson L-E. High prevalence of hypertension in sleep apnea patients independent of obesity. Am J Respir Crit Care Med. 1994;150(1):72-77.

364. Priou P, Le Vaillant M, Meslier N, et al. Cumulative association of obstructive sleep apnea severity and short sleep duration with the risk for hypertension. PLoS One. 2014;9(12):e115666.

365. Nieto FJ, Young TB, Lind BK, et al. Association of sleep-disordered breathing, sleep apnea, and hypertension in a large community-based study. JAMA. 2000;283(14):1829-1836.

366. O'Connor GT, Caffo B, Newman AB, et al. Prospective study of sleep-disordered breathing and hypertension: the Sleep Heart Health Study. Am J Respir Crit Care Med. 2009;179(12):1159-1164.

367. Cano-Pumarega I, Durán-Cantolla J, Aizpuru F, et al. Obstructive sleep apnea and systemic hypertension: longitudinal study in the general population: the Vitoria Sleep Cohort. Am J Respir Crit Care Med. 2011;184(11):1299-1304.

368. Torres G, Sánchez-de-la-Torre M, Barbé F. Relationship between OSA and hypertension. Chest. 2015;148(3):824-832.

369. Bourjeily G, Fung JY, Sharkey KM, et al. Airflow limitations in pregnant women suspected of sleepdisordered breathing. Sleep Med. 2014;15(5):550-555.

370. Young T, Evans L, Finn L, Palta M. Estimation of the clinically diagnosed proportion of sleep apnea syndrome in middle-aged men and women. Sleep. 1997;20(9):705-706.

371. Marin JM, Agusti A, Villar I, et al. Association between treated and untreated obstructive sleep apnea and risk of hypertension. JAMA. 2012;307(20):2169-2176. 
372. Ancoli-Israel S, Stepnowsky C, Dimsdale J, Marler M, Cohen-Zion M, Johnson S. The effect of race and sleep-disordered breathing on nocturnal BP "dipping": analysis in an older population. Chest. 2002;122(4):1148-1155.

373. Cho JS, Ihm SH, Kim CJ, et al. Obstructive sleep apnea using Watch-PAT 200 is independently associated with an increase in morning blood pressure surge in never-treated hypertensive patients. $J$ Clin Hypertens. 2015;17(9):675-681.

374. Noda A, Okada T, Hayashi H, Yasuma F, Yokota M. 24-hour ambulatory blood pressure variability in obstructive sleep apnea syndrome. Chest. 1993;103(5):1343-1347.

375. Lavie P, Yoffe N, Berger I, Peled R. The relationship between the severity of sleep apnea syndrome and 24-h blood pressure values in patients with obstructive sleep apnea. Chest. 1993;103(3):717-721.

376. Pankow W, Nabe B, Lies A, Kohl F, Lohmann F. Influence of obstructive sleep apnoea on circadian blood pressure profile. J Sleep Res. 1995;4(s1):102-106.

377. Hedner J, Bengtsson-Boström K, Peker Y, Grote L, Råstam L, Lindblad U. Hypertension prevalence in obstructive sleep apnoea and sex: a population-based case-control study. Eur Respir J. 2006;27(3):564-570.

378. Leke J, Fletcher EC, Bao G, Unger T. Hypertension caused by chronic intermittent hypoxia-influence of chemoreceptors and sympathetic nervous system. J Hypertens. 1997;15(12):1593-1603.

379. Zoccal DB, Bonagamba LG, Oliveira FR, Antunes-Rodrigues J, Machado BH. Increased sympathetic activity in rats submitted to chronic intermittent hypoxia. Exp Physiol. 2007;92(1):79-85.

380. Fletcher EC, Lesske J, Behm R, Miller CC, Stauss H, Unger T. Carotid chemoreceptors, systemic blood pressure, and chronic episodic hypoxia mimicking sleep apnea. J Appl Physiol. 1992;72(5):1978-1984.

381. Duckitt K, Harrington D. Risk factors for pre-eclampsia at antenatal booking: systematic review of controlled studies. BMJ. 2005;330(7491):565.

382. Larry $C D$, Yeo S. The circadian rhythm of blood pressure during pregnancy. J Obstet Gynecol Neonatal Nurs. 2000;29(5):500-508.

383. Benedetto C, Zonca M, Marozio L, Dolci C, Carandente F, Massobrio M. Blood pressure patterns in normal pregnancy and in pregnancy-induced hypertension, preeclampsia, and chronic hypertension. Obstet Gynecol. 1996;88(4):503-510.

384. Katragadda S, Xie A, Puleo D, Skatrud JB, Morgan BJ. Neural mechanism of the pressor response to obstructive and nonobstructive apnea. J Appl Physiol. 1997;83(6):2048-2054.

385. Wolf $\mathrm{J}$, Hering D, Narkiewicz K. Non-dipping pattern of hypertension and obstructive sleep apnea syndrome. Hypertens Res. 2010;33(9):867-871.

386. Young T, Peppard P, Palta M, et al. Population-based study of sleep-disordered breathing as a risk factor for hypertension. Arch Intern Med. 1997;157(15):1746-1752.

387. Hla KM, Young TB, Bidwell T, Palta M, Skatrud JB, Dempsey J. Sleep apnea and hypertension. Ann Intern Med. 1994;120(5):382-388.

388. Pankow W, Nabe B, Lies A, et al. Influence of sleep apnea on 24-hour blood pressure. Chest. 1997;112(5):1253-1258.

389. Pierdomenico SD, Pierdomenico AM, Cuccurullo F. Morning blood pressure surge, dipping, and risk of ischemic stroke in elderly patients treated for hypertension. Am J Hypertens. 2013;27(4):564-570.

390. Pierdomenico SD, Pierdomenico AM, Di Tommaso R, et al. Morning blood pressure surge, dipping, and risk of coronary events in elderly treated hypertensive patients. Am J Hypertens. 2015;29(1):39-45.

391. Amodeo C, Guimarães GG, Picotti JC, et al. Morning blood pressure surge is associated with death in hypertensive patients. Blood Press Monit. 2014;19(4):199-202.

392. Pickering G, Sleight $P$, Smyth $H$. The relation of arterial pressure to sleep and arousal in man. J Physiol. 1967;191(2):76P-78P.

393. Turjanmaa V, Kalli S, Majahalme S, Saranummi N, Uusitalo A. Diurnal blood pressure profiles and variability in normotensive ambulant subjects. Clin Physiol Funct Imaging. 1987;7(5):389-401.

394. Peled N, Greenberg A, Pillar G, Zinder O, Levi N, Lavie P. Contributions of hypoxia and respiratory disturbance index to sympathetic activation and blood pressure in obstructive sleep apnea syndrome. Am J Hypertens. 1998;11(11):1284-1289.

395. Tkacova R, McNicholas WT, Javorsky M, et al. Nocturnal intermittent hypoxia predicts prevalent hypertension in the European Sleep Apnoea Database cohort study. Eur Respir J. 2014;44(4):931-941.

396. Ali N, Davies R, Fleetham J, Stradling J. The acute effects of continuous positive airway pressure and oxygen administration on blood pressure during obstructive sleep apnea. Chest. 1992;101(6):15261532.

397. Bonsignore M, Marrone O, Insalaco G, Bonsignore G. The cardiovascular effects of obstructive sleep apnoeas: analysis of pathogenic mechanisms. Eur Respir J. 1994;7(4):786-805. 
398. Lackman F, Capewell V, Richardson B, daSilva O, Gagnon R. The risks of spontaneous preterm delivery and perinatal mortality in relation to size at birth according to fetal versus neonatal growth standards. Am J Obstet Gynecol. 2001;184(5):946-953.

399. Bernstein IM, Horbar JD, Badger GJ, Ohlsson A, Golan A. Morbidity and mortality among very-lowbirth-weight neonates with intrauterine growth restriction. Am J Obstet Gynecol. 2000;182(1):198-206.

400. McIntire DD, Bloom SL, Casey BM, Leveno KJ. Birth weight in relation to morbidity and mortality among newborn infants. N Engl J Med. 1999;340(16):1234-1238.

401. Pallotto EK, Kilbride HW. Perinatal outcome and later implications of intrauterine growth restriction. Clin Obstet Gynecol. 2006;49(2):257-269.

402. Gardosi J, Kady SM, McGeown P, Francis A, Tonks A. Classification of stillbirth by relevant condition at death (ReCoDe): population based cohort study. BMJ. 2005;331(7525):1113-1117.

403. Cockerill R, Whitworth MK, Heazell AEP. Do medical certificates of stillbirth provide accurate and useful information regarding the cause of death? Paediatr Perinat Epidemiol. 2012;26(2):117-123.

404. Randolph DA, Nolen TL, Ambalavanan N, et al. Outcomes of extremely low birthweight infants with acidosis at birth. Arch Dis Child Fetal Neonatal Ed. 2014;99(4):F263-268.

405. The Royal Australian and New Zealand College of Obstetricians and Gynaecologists. Fetal Physiology. 2017 [cited 2017 December 12]; Available from: https://ofsep.fsep.edu.au/mod/book/view.php?id=403

406. Martin CB, Jr. Normal fetal physiology and behavior, and adaptive responses with hypoxemia. Semin Perinatol. 2008;32(4):239-242.

407. Flenady V, Middleton P, Smith GC, et al. Stillbirths: the way forward in high-income countries. Lancet. 2011;377(9778):1703-1717.

408. Gardosi J, Madurasinghe V, Williams M, Malik A, Francis A. Maternal and fetal risk factors for stillbirth: population based study. BMJ. 2013;346:f108.

409. Mendez-Figueroa H, Pedroza C, Khan AM, Chauhan SP. Small-for-gestational-age infants among uncomplicated pregnancies at term: a secondary analysis of 9 Maternal-Fetal Medicine Units Network studies. Am J Obstet Gynecol. 2016;215(5):628.e1-e7.

410. Vashevnik S, Walker S, Permezel M. Stillbirths and neonatal deaths in appropriate, small and large birthweight for gestational age fetuses. Aust N Z J Obstet Gynaecol. 2007;47(4):302-306.

411. Hepburn M, Rosenberg K. An audit of the detection and management of small-for-gestational age babies. Br J Obstet Gynaecol. 1986;93(3):212-216.

412. Edelstone DI. Fetal compensatory responses to reduced oxygen delivery. Semin Perinatol. 1984;8(3):184-191.

413. Joel-Cohen SJ, Schoenfeld A. Fetal response to periodic sleep apnea during pregnancy: a new syndrome in obstetrics. Eur J Obstet Gynecol Reprod Biol. 1978;877-881.

414. Rurak D, Richardson BS, Patrick JE, Carmichael L, Homan J. Oxygen consumption in the fetal lamb during sustained hypoxemia with progressive acidemia. Am J Physiol. 1990;258(5):R1108-R1115.

415. Bocking A, White S, Homan J, Richardson B. Oxygen consumption is maintained in fetal sheep during prolonged hypoxaemia. J Dev Physiol. 1992;17(4):169-174.

416. Richardson B, Rurak D, Patrick J, Homan J, Carmichael L. Cerebral oxidative metabolism during sustained hypoxaemia in fetal sheep. J Dev Physiol. 1989;11(1):37-43.

417. Bernstein IM, Horbar JD, Badger GJ, Ohlsson A, Golan A. Morbidity and mortality among very-lowbirth-weight neonates with intrauterine growth restriction. The Vermont Oxford Network. Am J Obstet Gynecol. 2000;182(1 Pt 1):198-206.

418. Craigo SD, Beach ML, Harvey-Wilkes KB, D'Alton ME. Ultrasound predictors of neonatal outcome in intrauterine growth restriction. Am J Perinatol. 1996;13(8):465-471.

419. Bukowski R, Hansen $\mathrm{NI}$, Willinger $\mathrm{M}$, et al. Fetal growth and risk of stillbirth: a population-based casecontrol study. PLoS Med. 2014;11(4):e1001633.

420. MacDonald TM, Hui L, Tong S, et al. Reduced growth velocity across the third trimester is associated with placental insufficiency in fetuses born at a normal birthweight: a prospective cohort study. BMC Med. 2017;15(1):164.

421. Macdonald-Wallis C, Tilling K, Fraser A, Nelson SM, Lawlor DA. Associations of blood pressure change in pregnancy with fetal growth and gestational age at delivery: findings from a prospective cohort. Hypertension. 2014;64(1):36-44.

422. Bakker R, Steegers EA, Hofman A, Jaddoe VW. Blood pressure in different gestational trimesters, fetal growth, and the risk of adverse birth outcomes: The Generation R Study. Am J Epidemiol. 2011;174(7):797-806.

423. Anderson NH, Sadler LC, Stewart AW, Fyfe EM, McCowan LM. Independent risk factors for infants who are small for gestational age by customised birthweight centiles in a multi-ethnic New Zealand population. Aust N Z J Obstet Gynaecol. 2013;53(2):136-142. 
424. Allen VM, Joseph K, Murphy KE, Magee LA, Ohlsson A. The effect of hypertensive disorders in pregnancy on small for gestational age and stillbirth: a population based study. BMC Pregnancy Childbirth. 2004;4(1):17.

425. Xiong $X$, Mayes $D$, Demianczuk N, et al. Impact of pregnancy-induced hypertension on fetal growth. Am J Obstet Gynecol. 1999;180(1 Pt 1):207-213.

426. Keyes LE, Armaza JF, Niermeyer S, Vargas E, Young DA, Moore LG. Intrauterine growth restriction, preeclampsia, and intrauterine mortality at high altitude in Bolivia. Pediatr Res. 2003;54(1):20-25.

427. Meschia G. Fetal oxygenation and maternal ventilation. Clin Chest Med. 2011;3215-3219.

428. Julian CG, Wilson MJ, Lopez M, et al. Augmented uterine artery blood flow and oxygen delivery protect Andeans from altitude-associated reductions in fetal growth. Am J Physiol Regul Integr Comp Physiol. 2009;296(5):R1564-1575.

429. Jensen GM, Moore LG. The effect of high altitude and other risk factors on birthweight: independent or interactive effects? Am J Public Health. 1997;87(6):1003-1007.

430. Julian CG, Vargas E, Armaza JF, Wilson MJ, Niermeyer S, Moore LG. High-altitude ancestry protects against hypoxia-associated reductions in fetal growth. Arch Dis Child Fetal Neonatal Ed. 2007;92(5):F372-377.

431. Mortola JP, Frappell PB, Aguero L, Armstrong K. Birth weight and altitude: a study in Peruvian communities. J Pediatr. 2000;136(3):324-329.

432. Hafner $\mathrm{E}$, Metzenbauer $\mathrm{M}$, Hofinger $\mathrm{D}$, et al. Placental growth from the first to the second trimester of pregnancy in SGA-foetuses and pre-eclamptic pregnancies compared to normal foetuses. Placenta. 2003;24(4):336-342.

433. Jansson T, Thordstein M, Kjellmer I. Placental blood flow and fetal weight following uterine artery ligation. Temporal aspects of intrauterine growth retardation in the guinea pig. Biol Neonate 1986;49(3):172-180.

434. Marconi AM, Paolini CL, Stramare L, et al. Steady state maternal-fetal leucine enrichments in normal and intrauterine growth-restricted pregnancies. Pediatr Res. 1999;46(1):114-119.

435. Mane SV, Gharpure VP, Merchant RH. Maternal heart disease and perinatal outcome. Indian Pediatr. 1993;30(12):1407-1411.

436. Gelson E, Curry R, Gatzoulis MA, et al. Effect of maternal heart disease on fetal growth. Obstet Gynecol. 2011;117(4):886-891.

437. Harman CR. Assessment of fetal health. In: Creasy RK, Resnik R, lams JD, eds. Maternal-Fetal Medicine: Principles and Practice. Philadelphia, PA: W. B. Saunders, 2004:357-401.

438. Pamidi S, Marc I, Simoneau G, et al. Maternal sleep-disordered breathing and the risk of delivering small for gestational age infants: a prospective cohort study. Thorax. 2016;71(8):719-725.

439. de Grauw TJ, Myers RE, Scott WJ. Fetal growth retardation in rats from different levels of hypoxia. Neonatology. 1986;49(2):85-89.

440. McAuliffe F, Kametas N, Krampl E, Ernsting J, Nicolaides K. Blood gases in pregnancy at sea level and at high altitude. BJOG. 2001;108(9):980-985.

441. Moore L, Shriver M, Bemis L, et al. Maternal adaptation to high-altitude pregnancy: an experiment of nature-a review. Placenta. 2004;25 Suppl A:S60-S71.

442. Constância M, Hemberger M, Hughes J, et al. Placental-specific IGF-II is a major modulator of placental and fetal growth. Nature. 2002;417(6892):945-948.

443. Iwamoto HS, Murray MA, Chernausek SD. Effects of acute hypoxemia on insulin-like growth factors and their binding proteins in fetal sheep. Am J Physiol. 1992;263(6 Pt 1):E1151-1156.

444. Tapanainen PJ, Bang P, Wilson K, Unterman TG, Vreman HJ, Rosenfeld RG. Maternal hypoxia as a model for intrauterine growth retardation: effects on insulin-like growth factors and their binding proteins. Pediatr Res. 1994;36(2):152-158.

445. Tazuke SI, Mazure NM, Sugawara J, et al. Hypoxia stimulates insulin-like growth factor binding protein 1 (IGFBP-1) gene expression in HepG2 cells: a possible model for IGFBP-1 expression in fetal hypoxia. Proc Natl Acad Sci U S A. 1998;95(17):10188-10193.

446. Reid J, Skomro R, Gjevre J, Cotton D, Ward H, Olatunbosun O. Fetal heart rate monitoring during nocturnal polysomnography. Clin Exp Obstet Gynecol. 2011;38(2):123-125.

447. MacDonald TM, McCarthy EA, Walker SP. Shining light in dark corners: diagnosis and management of late-onset fetal growth restriction. Aust N Z J Obstet Gynaecol. 2015;55(1):3-10.

448. Mamelle N, Cochet $\mathrm{V}$, Claris $\mathrm{O}$. Definition of fetal growth restriction according to constitutional growth potential. Biol Neonate 2001;80(4):277-285.

449. Gardosi J, Mul T, Mongelli M, Fagan D. Analysis of birthweight and gestational age in antepartum stillbirths. Br J Obstet Gynaecol. 1998;105(5):524-530.

450. Prior $T$, Paramasivam $G$, Bennett $P$, Kumar $S$. Are fetuses that fail to achieve their growth potential at increased risk of intrapartum compromise? Ultrasound Obstet Gynecol. 2015;46(4):460-464. 
451. Gardosi J. New definition of small for gestational age based on fetal growth potential. Horm Res. 2006;65 Suppl 3:15-18.

452. Hadlock FP, Harrist RB, Martinez-Poyer J. In utero analysis of fetal growth: a sonographic weight standard. Radiology. 1991;181(1):129-133.

453. Smith-Bindman R, Chu PW, Ecker JL, Feldstein VA, Filly RA, Bacchetti P. US evaluation of fetal growth: prediction of neonatal outcomes. Radiology. 2002;223(1):153-161.

454. Bardien N, Whitehead CL, Tong S, Ugoni A, McDonald S, Walker SP. Placental insufficiency in fetuses that slow in growth but are born appropriate for gestational age: a prospective longitudinal study. PLoS One. 2016;11(1):e0142788.

455. Manconi M, Ulfberg J, Berger K, et al. When gender matters: restless legs syndrome. Report of the "RLS and woman" workshop endorsed by the European RLS Study Group. Sleep Med Rev. 2012;16(4):297-307.

456. Montplaisir J, Allen RP, Walters A, Ferini-Strambi L. Restless legs syndrome and periodic limb movements during sleep. In: Kryger M, Roth T, Dement WC, eds. Principles and Practice of Sleep Medicine. 5th ed. Philadelphia: W. B. Saunders, 2011.

457. Izci Balserak B, Lee K. Sleep disturbances and sleep-related disorders in pregnancy. In: Kryger M, Roth T, Dement WC, eds. Principles and Practice of Sleep Medicine. 5th ed. Philadelphia, Pennsylvania: W. B. Saunders, 2011.

458. Sarberg M, Josefsson A, Wirehn A, Svanborg E. Restless legs syndrome during and after pregnancy and its relation to snoring. Acta Obstet Gynecol Scand. 2012;91(7):850-855.

459. American Academy of Sleep Medicine. International classification of sleep disorders. 3rd ed. Darien, IL: American Academy of Sleep Medicine, 2014.

460. Lee KA, Zaffke ME, Baratte-Beebe K. Restless legs syndrome and sleep disturbance during pregnancy: the role of folate and iron. J Womens Health Gend Based Med. 2001;10(4):335-341.

461. Berry RB, Budhiraja R, Gottlieb DJ, et al. Rules for scoring respiratory events in sleep: update of the 2007 AASM Manual for the Scoring of Sleep and Associated Events. Deliberations of the Sleep Apnea Definitions Task Force of the American Academy of Sleep Medicine. J Clin Sleep Med. 2012;8(5):597619.

462. Scofield $\mathrm{H}$, Roth $\mathrm{T}$, Drake $\mathrm{C}$. Periodic limb movements during sleep: population prevalence, clinical correlates, and racial differences. Sleep. 2008;31(9):1221-1227.

463. Pennestri M, Whittom S, Adam B, Petit D, Carrier J, Montplaisir J. PLMS and PLMW in healthy subjects as a function of age: prevalence and interval distribution. Sleep. 2006;29(9):1183-1187.

464. Haba-Rubio J, Marti-Soler H, Marques-Vidal P, et al. Prevalence and determinants of periodic limb movements in the general population. Ann Neurol. 2016;79(3):464-474.

465. Carrier J, Frenette S, Montplaisir J, Paquet J, Drapeau C, Morettini J. Effects of periodic leg movements during sleep in middle-aged subjects without sleep complaints. Mov Disord. 2005;20(9):1127-1132.

466. Mendelson WB. Are periodic leg movements associated with clinical sleep disturbance? Sleep. 1996;19(3):219-223.

467. Youngstedt SD, Kripke DF, Klauber MR, Sepulveda RS, Mason WJ. Periodic leg movements during sleep and sleep disturbances in elders. J Gerontol A Biol Sci Med Sci. 1998;53(5):M391-M394.

468. Bastuji H, García-Larrea L. Sleep/wake abnormalities in patients with periodic leg movements during sleep: Factor analysis on data from 24-h ambulatory polygraphy. J Sleep Res. 1999;8(3):217-223.

469. Hilbert J, Mohsenin V. Can periodic limb movement disorder be diagnosed without polysomnography? A case-control study. Sleep Med. 2003;4(1):35-41.

470. Pennestri M-H, Montplaisir J, Fradette L, Lavigne G, Colombo R, Lanfranchi PA. Blood pressure changes associated with periodic leg movements during sleep in healthy subjects. Sleep Med. 2013;14(6):555-561.

471. Ohayon MM, Roth T. Prevalence of restless legs syndrome and periodic limb movement disorder in the general population. J Psychosom Res. 2002;53(1):547-554.

472. Ulfberg J, Nyström B, Carter N, Edling C. Prevalence of restless legs syndrome among men aged 18 to 64 years: an association with somatic disease and neuropsychiatric symptoms. Mov Disord. 2001;16(6):1159-1163.

473. Espinar-Sierra J, Vela-Bueno A, Luque-Otero M. Periodic leg movements in sleep in essential hypertension. Psychiatry Clin Neurosci. 1997;51(3):103-107.

474. Sieminski M, Partinen M. A relationship between periodic limb movements in sleep and high nocturnal blood pressure values in patients with insomnia. J Clin Sleep Med. 2016;12(6):865-869.

475. Mirza M, Shen W-K, Sofi A, et al. Frequent periodic leg movement during sleep is associated with left ventricular hypertrophy and adverse cardiovascular outcomes. J Am Soc Echocardiogr. 2013;26(7):783-790. 
476. Hanly PJ, Zuberi-Khokhar N. Periodic limb movements during sleep in patients with congestive heart failure. Chest. 1996;109(6):1497-1502.

477. Yumino D, Wang $\mathrm{H}$, Floras JF, et al. Relation of periodic leg movements during sleep and mortality in patients with systolic heart failure. Am J Cardiol. 2011;107(3):447-451.

478. Nannapaneni S, Ramar K. Periodic limb movements during sleep and their effect on the cardiovascular system: is there a final answer? Sleep Med. 2014;15(4):379-384.

479. Hornyak M, Feige B, Riemann D, Voderholzer U. Periodic leg movements in sleep and periodic limb movement disorder: prevalence, clinical significance and treatment. Sleep Med Rev. 2006;10(3):169177.

480. Earley C, Connor J, Beard J, Malecki E, Epstein D, Allen R. Abnormalities in CSF concentrations of ferritin and transferrin in restless legs syndrome. Neurology. 2000;54(8):1698-1700.

481. Picchietti DL, Hensley JG, Bainbridge JL, et al. Consensus clinical practice guidelines for the diagnosis and treatment of restless legs syndrome/Willis-Ekbom disease during pregnancy and lactation. Sleep Med Rev. 2015;22:64-77.

482. Hornyak M, Kopasz M, Feige B, Riemann D, Voderholzer U. Variability of periodic leg movements in various sleep disorders: implications for clinical and pathophysiologic studies. Sleep. 2005;28(3):331335.

483. Bliwise DL, Carskadon MA, Dement WC. Nightly variation of periodic leg movements in sleep in middle aged and elderly individuals. Arch Gerontol Geriatr. 1988;7(4):273-279.

484. Sforza E, Haba-Rubio J. Night-to-night variability in periodic leg movements in patients with restless legs syndrome. Sleep Med. 2005;6(3):259-267.

485. Hening WA, Allen RP, Washburn M, Lesage SR, Earley CJ. The four diagnostic criteria for Restless Legs Syndrome are unable to exclude confounding conditions ("mimics"). Sleep Med. 2009;10(9):976981.

486. Allen RP, Picchietti DL, Garcia-Borreguero D, et al. Restless legs syndrome/Willis-Ekbom disease diagnostic criteria: updated International Restless Legs Syndrome Study Group (IRLSSG) consensus criteria-history, rationale, description, and significance. Sleep Med. 2014;15(8):860-873.

487. Picchietti D. Periodic limb movements in sleep: irrelevant epiphenomenon, marker for a potential problem, or a disorder. J Clin Sleep Med. 2006;2(4):446-447.

488. Louis J, Auckley D, Bolden N. Management of obstructive sleep apnea in pregnant women. Obstet Gynecol. 2012;119(4):864-868.

489. Pien GW, Schwab RJ. Sleep disorders during pregnancy. Sleep. 2004;27(7):1405-1417.

490. Wolf M, Kettyle E, Sandler L, Ecker JL, Roberts J, Thadhani R. Obesity and preeclampsia: the potential role of inflammation. Obstet Gynecol. 2001;98(5):757-762.

491. Flenady V, Koopmans L, Middleton P, et al. Major risk factors for stillbirth in high-income countries: a systematic review and meta-analysis. Lancet. 2011;377(9774):1331-1340.

492. Chu SY, Kim SY, Lau J, et al. Maternal obesity and risk of stillbirth: a metaanalysis. Am J Obstet Gynecol. 2007;197(3):223-228.

493. O'Donoghue FJ, Catcheside PG, Ellis E, et al. Sleep hypoventilation in hypercapnic chronic obstructive pulmonary disease: prevalence and associated factors. Eur Respir J. 2003;21(6):977-984.

494. Aittokallio T, Saaresranta T, Polo-Kantola P, Nevalainen O, Polo O. Analysis of inspiratory flow shapes in patients with partial upper-airway obstruction during sleep. Chest. 2001;119(1):37-44.

495. Correa P, Palmeiro Y, Soto M, Ugarte C, Illanes S. Etiopathogenesis, prediction, and prevention of preeclampsia. Hypertens Pregnancy. 2016;35(3):280-294.

496. Leslie K, Thilaganathan B, Papageorghiou A. Early prediction and prevention of pre-eclampsia. Best Pract Res Clin Obstet Gynaecol. 2011;25(3):343-354.

497. Stiasny K, Oertel WH, Trenkwalder C. Clinical symptomatology and treatment of restless legs syndrome and periodic limb movement disorder. Sleep Med Rev. 2002;6(4):253-265.

498. Golbe LI. Pregnancy and movement disorders. Neurol Clin. 1994;12(3):497-508.

499. Bin YS, Cistulli PA, Ford JB. Population-based study of sleep apnea in pregnancy and maternal and infant outcomes. J Clin Sleep Med. 2016;12(6):871-877.

500. Gordon A, Raynes-Greenow C, Bond D. Sleep position, fetal growth restriction, and late-pregnancy stillbirth. Obstet Gynecol. 2015;125(2):347-355.

501. Farine D, Seaward PG. When it comes to pregnant women sleeping, is left right? J Obstet Gynaecol Can. 2007;29(10):841-842.

502. Joosten SA, O'Driscoll DM, Berger PJ, Hamilton GS. Supine position related obstructive sleep apnea in adults: pathogenesis and treatment. Sleep Med Rev. 2014;18(1):7-17.

503. Bamber JH, Dresner M. Aortocaval compression in pregnancy: the effect of changing the degree and direction of lateral tilt on maternal cardiac output. Anesth Analg. 2003;97(1):256-258. 
504. Chen GY, Kuo CD, Yang MJ, Lo HM, Tsai YS. Comparison of supine and upright positions on autonomic nervous activity in late pregnancy: the role of aortocaval compression. Anaesthesia. 1999;54(3):215219.

505. Clark SL, Cotton DB, Pivarnik JM, et al. Position change and central hemodynamic profile during normal third-trimester pregnancy and post partum. Am J Obstet Gynecol. 1991;164(3):883-887.

506. Ellington C, Katz VL, Watson WJ, Spielman FJ. The effect of lateral tilt on maternal and fetal hemodynamic variables. Obstet Gynecol. 1991;77(2):201-203.

507. Kauppila A, Koskinen M, Puolakka J, Tuimala R, Kuikka J. Decreased intervillous and unchanged myometrial blood flow in supine recumbency. Obstet Gynecol. 1980;55(2):203-205.

508. Milsom I, Forssman L. Factors influencing aortocaval compression in late pregnancy. Am J Obstet Gynecol. 1984;148(6):764-771.

509. Jeffreys R, Stepanchak W, Lopez B, Hardis J, Clapp J. Uterine blood flow during supine rest and exercise after 28 weeks of gestation. BJOG. 2006;113(11):1239-1247.

510. Khatib N, Weiner Z, Beloosesky R, Vitner D, Thaler I. The effect of maternal supine position on umbilical and cerebral blood flow indices. Eur J Obstet Gynecol Reprod Biol. 2014;175:112-114.

511. Warland J, Dorrian J. Accuracy of self-reported sleep position in late pregnancy. PLoS One. 2014;9(12):e115760. 
Appendix A

Participant Information and Consent Form 


\title{
PARTICIPANT INFORMATION AND CONSENT FORM
}

\author{
SLEEP-DISORDERED BREATHING IN GESTATIONAL HYPERTENSION AND \\ PREECLAMPSIA: IMPACT ON MATERNAL AND FETAL OUTCOMES
}

\section{Project Number:}

\author{
Principal Researcher: \\ Associate Researchers:
}

\author{
H2012/04469 (Austin Health) R12/02 (Mercy Health)
}

Prof. Sue Walker and Dr. Mark Howard.

Ms. Danielle Wilson, Dr. Fergal O’Donoghue, Dr. Maree

Barnes, Dr. Alison Fung, Dr. Martha Lappas.

\section{Introduction}

You are invited to take part in a research project looking at the relationship between sleep health and its effect on pregnancy and the unborn baby. We are especially interested in how sleep-related problems affect women diagnosed with high blood pressure or preeclampsia during pregnancy. Preeclampsia is a serious medical disorder of pregnancy and causes several problems such as high blood pressure, leakage of protein into the urine, thinning of the blood and liver dysfunction. You have been approached to participate in this study either because you have been diagnosed with high blood pressure, or because you have a healthy and uncomplicated pregnancy (i.e. which we wish to use as a healthy comparison). This Participant Information and Consent Form tells you about the research project. It explains the procedures involved. Knowing what is involved will help you decide if you want to take part in the research.

Please read this information carefully. Ask questions about anything that you don't understand or want to know more about. Before deciding whether or not to take part, you might want to talk about it with a relative, friend, or healthcare worker.

Participation in this research is voluntary. If you don't wish to take part, you don't have to. You will receive the best possible care whether you take part or not.

If you decide you want to take part in the research project, you will be asked to sign the consent section. By signed it you are telling us that you:

- Understand what you have read;

- Consent to take part in the research project;

- Consent to participate in the research processes that are described;

- Consent to the use of your personal and health information as described.

You will be given a copy of the Participant Information and Consent Form to keep.

\section{Purpose and Background}

Sleep complaints, including snoring, are commonly reported in late pregnancy. Snoring is a sound caused by the vibration of loose tissue in the upper airway, and can also be a sign of sleep-disordered breathing, or a condition called obstructive sleep apnoea (OSA). OSA is a condition in which a person's breathing stops or is reduced for several seconds at a time, many times over, during sleep. During pregnancy, weight gain and elevation of the diaphragm from the growing uterus may increase the risk of sleep-disordered breathing.

In the general population, snoring and OSA have been associated with the development of conditions such as high blood pressure and other long-term health problems. However, it is unknown how commonly snoring and OSA actually occur during pregnancy, and whether there are any consequences to the mother or her baby.

Preeclampsia and high blood pressure in pregnancy (the development of high blood pressure in a pregnant woman after 20 weeks gestation is known as gestational hypertension) are important 
disorders of pregnancy which can increase the risk of poor outcomes for the mother and baby. Snoring and OSA have been shown to be more common in gestational hypertension and preeclampsia, and it is possible that the effects of these sleep disorders may magnify the already negative consequences of hypertension and preeclampsia. Importantly, if this study can show that snoring and OSA have an additional negative impact on women with hypertension or preeclampsia and their babies, then a trial involving the treatment of snoring and OSA may enable these high risk pregnancies to safely continue later into gestation, thereby improving outcomes.

The first purpose of this study is to see whether snoring and signs of OSA are more common in women diagnosed with hypertension or preeclampsia during pregnancy, compared to women without this diagnosis. The second purpose of this study is to determine whether the presence of snoring or OSA during pregnancy has any effect on the health of the mother and baby, particularly for women diagnosed with hypertension or preeclampsia.

All participants will receive feedback on their sleep patterns from this research. You are invited to participate in this research project because there is a lack of studies looking at the consequences of sleep problems during pregnancy. The identification of any potential health problems during pregnancy is of the utmost importance, especially if there is a potential treatment already available.

A total of approximately 180 pregnant women from the Mercy Hospital for Women will participate in this project. This project is being conducted collaboratively between the Mercy Hospital for Women and the Institute for Breathing and Sleep at the Austin Hospital. Ms. Danielle Wilson will submit this research to University of Melbourne as the basis for the degree of Doctor of Philosophy (PhD). This research project has also received funding from the Austin Medical Research Foundation.

\section{What does participation in this research project involve?}

This project will involve 2 groups of women:

1.Pregnant women with a diagnosis of hypertension or preeclampsia (participants with this condition)

2. Pregnant women without a diagnosis of hypertension or preeclampsia (participants without this condition)

This study will involve three to four hospital visits for all participants. These visits may coincide with your routine antenatal care, when you would already have a scheduled hospital appointment.

For your first visit at approximately 28-34 weeks gestation, we will invite you to attend an interview at the Mercy Hospital for Women so we can fully explain to you what is involved in the research project. After signing the informed consent form on page 7 of this document, a blood sample will be taken. An ultrasound will be performed to monitor the growth of your baby. We will not reveal the sex of your baby unless you specifically ask, and we can only be $95 \%$ confident of accurately identifying the baby's sex. This appointment should take approximately 45 minutes.

Usually within 2 weeks of enrolling in the study, you will be invited to undertake an overnight sleep study. On arrival at the Austin Hospital sleep laboratory, you will be met by one of the researchers. You will be asked to fill out a few questionnaires about your sleep health, and will be asked to take a copy of two of the questionnaires home for your partner to fill out confidentially. Overnight you will be set-up with the sleep monitoring equipment, and you will also have your blood pressure and your baby's heart rate monitored while you sleep. You will be allocated a private room in the sleep laboratory to sleep undisturbed overnight, and when you wake in the morning you will be detached from the monitoring equipment and allowed to go home. Every participant in this research will receive feedback on their sleep patterns once analysed.

If you are yet to deliver your baby four weeks later, you will be invited to undergo a second overnight sleep study with your blood pressure and your baby's heart rate monitored. Your decision whether or not to have this second sleep study will not affect your overall participation in this study or your relationship with your treating doctors.

The last visit for this research study involves sampling of your blood and the umbilical cord blood when you deliver your baby at the Mercy Hospital for Women. With your consent, we will also gather 
information from your medical history about your pregnancy and your baby such as birth weight and APGAR scores, and measures related to your hypertension or preeclampsia diagnosis and treatment.

The APGAR score is a method of assessing the baby's overall status and response to resuscitation. It is based on the assessment of heart rate, respiratory effort, muscle tone, reflex irritability and colour.

You will not be paid for your participation in this research, but you will be reimbursed for any of the travel costs that you incur as a result of additional hospital visits for participating in this research (i.e. $\$ 30$ taxi fare for each visit, public transport costs, hospital parking costs).

\section{What are the tests?}

\section{A. Questionnaires}

i) MAP Index, Berlin Questionnaire and SASQ

These three brief questionnaires will ask you about the frequency of various symptoms of sleep, such as snoring and tiredness. They will take approximately 10 minutes to complete.

ii) Restless Legs Syndrome Questionnaire

This brief questionnaire is designed to screen for the presence of Restless Legs Syndrome, which is a condition involving the urge to move the limbs usually associated with uncomfortable or unpleasant sensations. This condition worsens during the night, and is thought to occur more often during pregnancy. This questionnaire will take less than 5 minutes to complete.

\section{B. Ultrasound - approximately 15 minutes}

The ultrasound is non-invasive and involves the placement of some gel on the abdominal skin to assist the probe with giving us a good image of your baby. These scans will monitor the growth of your baby. The ultrasound is performed by an experienced Consultant Obstetrician. Any abnormal results will be managed clinically as per standard practice.

\section{Overnight Sleep Monitoring}

\section{i) Sleep Study}

You will be asked to arrive at the sleep laboratory at the Austin Hospital at approximately 7:30pm in the evening, and the sleep study will finish at approximately 6.30am the next morning.

When you come in, you will be met by the researcher and shown to your private room. Bathroom facilities are shared. There is a small lounge/television room for your use, and microwave / fridge facilities are available. You should have your dinner before coming to the hospital, bring night attire, toiletries, and are welcome to bring your own pillow. You should bring all your own medication and take any medication as you would normally. Don't drink coffee, tea, coke or alcohol from $4 \mathrm{pm}$ on the afternoon of the overnight study. If you wish, you may bring non-caffeinated drinks with you to the hospital. Alcohol should be avoided from 12 midday on the day of this study.

The researcher will explain the equipment and procedures to you, then will attach several electrodes to your head, face, chest and legs to monitor your heart and the activity of your brain, your eyes, and the muscles of your face and legs. You will also have 2 bands strapped around your chest and abdomen to monitor your breathing, an airflow detector attached to your nose and an oxygen sensor attached to a finger. All of the sensors will be plugged into a portable monitor, which will attach to the band around your chest. This may sound very uncomfortable and restrictive, but none of the sensors are invasive or painful, and you are able to walk around, read, watch television, eat and drink. You will remain under the care of the sleep scientists overnight, but will be left to sleep undisturbed. In the morning, all of the sensors will be detached and you will be free to leave.

Following your sleep study, the researchers will give you feedback on your results. If your test results show that you have obstructive sleep apnoea, we can refer you to a Sleep Specialist within the Austin Hospital for follow-up. 
ii) $\quad$ CTG (Cardiotocography) Monitoring

The researcher will attach this non-invasive portable monitor to your abdomen using five sticky electrodes on the surface of your skin. This device will monitor the fetal heart rate continuously overnight. The data will be analysed first thing in the morning by a consultant obstetrician, and should there be any concerns you will be reviewed by one of the study investigators trained in obstetric medicine.

iii) Blood Pressure Monitoring

The researcher will attach this non-invasive portable monitor to two fingers on one hand. The finger cuffs will inflate at alternating intervals during the night to continuously monitor your blood pressure. Sleep is rarely disturbed by this device and no discomfort is usually reported.

\section{Measure of Oxidative Stress}

A blood sample of $10 \mathrm{ml}$ (about $1 / 2$ tablespoon) will be taken on your first visit by the research assistant or pathology collector at the Mercy Hospital for Women. Another $10 \mathrm{ml}$ blood sample from yourself and the cord blood from the placenta will be collected by midwives at the time of delivery of your baby. This sample will be used to look for markers associated with preeclampsia and hormonal regulators of baby's growth that may be affected by snoring or OSA.

\section{What will happen to my test samples?}

You will be asked to provide additional consent for the collection, storage and use of your blood samples during the research project. The tests that will be performed on your samples will be used for this research study, or related studies examining sleep-disordered breathing and important pregnancy outcomes. A small amount of blood $(10 \mathrm{ml}$ or $1 / 2$ tablespoon) will be sampled from the inside of your elbow by a trained researcher or pathology collector. At delivery, a small amount of cord blood $(10 \mathrm{ml}$ or $1 / 2$ tablespoon) will be sampled from the placenta by the midwives. All samples will be handled by the University Department of Obstetrics and Gynaecology at the Mercy Hospital for Women and will be stored in their research laboratory according to their normal procedures. Data from the blood samples will be entered in a coded manner into our research study database. Thus all information regarding the sample will be recorded using a participant ID number only. The sample will be stored for 15 years and will be destroyed at the end of this period.

\section{What are the possible benefits?}

There will be no direct benefit to you for participating in this trial. You will not be paid for your participation in this trial.

\section{What are the possible risks?}

Possible risks, side effects and discomforts include

1) The main discomfort will be the time commitment we are asking of you, which includes an overnight stay in the sleep laboratory.

2) Blood test - having a blood sample taken may cause some discomfort of bruising. Sometimes, the blood vessel may swell, or blood may clot in the blood vessel, or the spot from which blood is taken could become inflamed. Rarely, there could be a minor infection or bleeding. If this happens, it can be easily treated.

3) During the sleep study, the cream and tapes used to attach the wires may cause some skin irritation.

4) If the ultrasound or overnight sleep testing does reveal any significant abnormalities, you will be referred to a specialist for further investigation.

If you become upset or distressed as a result of your participation in the research, the researcher is able to arrange for counselling or other appropriate support. Any counselling or support will be provided by staff who are not members of the research team. In addition, you may prefer to suspend or end your participation in the research if distress occurs.

There may be additional risks that the researchers do not expect or do not know about. Tell a member of the research team immediately about any new or unusual symptoms that you get. 


\section{What if new information arises during this research project?}

During the research project, new information about the risks and benefits of the project may become known to the researchers. If this occurs, you will be told about this new information and the researcher will discuss whether this new information affects you.

\section{Can I have other treatments during this research project?}

It is important to tell your doctor and the research staff about any treatments or medications you may be taking, including non-prescription medications, vitamins or herbal remedies, acupuncture or other alternative treatments. You should also tell your doctor about any changes to these during your participation in the research.

\section{Do I have to take part in this research project?}

Participation in any research project is voluntary. If you do not wish to take part you are not obliged to. If you decide to take part and later change your mind, you are free to withdraw from the project at any stage. Your decision whether to take part or not to take part, or to take part and then withdraw, will not affect your routine treatment, your relationship with those treating you or your relationship with Mercy Hospital for Women or Austin Health.

\section{What if I withdraw from this project?}

If you decide to withdraw from this project, please notify a member of the research team before you withdraw. This notice will allow that person or the research supervisor to inform you if there are any health risks or special requirements linked to withdrawing.

If you decide to leave the project, the researchers would like to keep the personal and health information about you that has been collected. This is to help them make sure that the results of the research can be measured properly. If you do not want them to do this, you can tell the researchers to withdraw your personal and health information and tissue samples from the project.

\section{How will I be informed of the results of this research project?}

At the end of the study you will receive a hard copy of your results by mail, and these can be further explained to you by one of the researchers by telephone or by appointment. We will happily send a copy to any doctor at your request.

\section{What will happen to information about me?}

Information about your participation in this research project may be recorded in your health records. Your results will not be shared with anyone else for any other purpose. The records dealing with this study will be kept in safe storage for 7 years, and then shredded. All records will be kept in coded form, and the code will only be accessible to the principal and associate researchers of this project.

Information about you may be obtained from your health records held at Mercy Hospital for Women and Austin Health for the purposes of this research. Any information obtained in connection with this research project that can identify you will remain confidential and will only be used for the purpose of this research project. It will only be disclosed with your permission, except as required by law. If you give us your permission by signing the Consent Form, we plan to publish the results in a scientific journal. In any publication, information will be provided in such a way that you cannot be identified.

In accordance with the National Medical Health and Research Council guidelines, the Human Research Ethics Committee is required to conduct audits of research projects from time to time. It may therefore be possible that the Human Research Ethics Committee which has approved this research, will seek to view a copy of your signed consent form, or to contact you, to ensure that the research is being conducted according to the ethical standards required by these guidelines. 
In accordance with the Freedom of Information Act 1982 (Vic), you have the right to access and to request correction of information held about you by Mercy Hospital for Women or Austin Health.

\section{Further Information or Any Problems}

Prof. Sue Walker and Dr. Mark Howard will be co-ordinating this study. If you have any questions or concerns, they can be contacted on:

Sue Walker: $\quad$ ph 84584248 during hours.

After hours: Mercy hospital switchboard, ph 8458-4444.

Mark Howard: ph 9496-3685 during hours.

After hours: Austin hospital switchboard, ph 9496-5000.

If you have any complaints about any aspect of the project, the way it is being conducted or any questions about your rights as a research participant, then you may contact:

- Ms Carole Branch, Administrative Officer, Mercy Health Human Research Ethics Committee on ph. 84584808 for referral of this concern to the Human Research Ethics Committee.

- Ms Jill Davis, Manager, Austin Health Research Ethics Unit. Ph: 94964034.

\section{ETHICAL GUIDELINES}

This project will be carried out according to the National Statement on Ethical Conduct in Human Research (2007) produced by the National Health and Medical Research Council of Australia. This statement has been developed to protect the interests of people who agree to participate in human research studies.

The ethical aspects of this research project have been approved by the Human Research Ethics Committee of Mercy Hospital for Women and Austin Health. 
CONSENT FORM TO PARTICIPATE IN RESEARCH

Project Title: Sleep-Disordered Breathing in Gestational Hypertension and Preeclampsia: Impact on Maternal and Fetal Outcomes

Project Number: H2012/04469 (Austin Health) \& R12/02 (Mercy Health)

I have read, or have had read to me in a language that I understand, this document and I understand the purposes, procedures and risks of this research project as described within it.

I give permission for my doctors, other health professionals, hospitals and laboratories outside this hospital to release information to the Mercy Hospital for Women and Austin Health concerning my disease and treatment that is needed for this project. I understand that such information will remain confidential.

I consent to the Mercy Health and Austin Health Human Research Ethics Committees which approved this study to access my information, or to contact me to ask about my research experience, in order to ensure that the project is being run in accordance with government standards.

I have had an opportunity to ask questions and I am satisfied with the answers I have received. I freely agree to participate in this research project as described. I understand that I will be given a signed copy of this document to keep.

I consent to the storage and use of blood and tissue samples taken from me for use in:

$\square$ this specific research project

$\square$ other research that is closely related to this research project

As described in Section 4 of this document by

Participant's name (printed)

Signature:Date:

Name of witness to participant's signature (printed)

Signature:Date:

Declaration by researcher: I have given a verbal explanation of the research project, its procedures and risks and I believe that the participant has understood that explanation.

Researcher's name (printed)

Signature:Date: 
Appendix B

The Berlin Questionnaire 


\section{Berlin Questionnaire}

Age years

Weight before pregnancy $\mathrm{kg}$

Height $\mathrm{cm}$

Please tick the most appropriate option in regards to the past month:

1. Do you snore?

$\square$ Yes $\quad \square$ No $\quad \square$ Don't know

If you do not snore, please go to Question 5.

If you do snore:

2. Is your snoring loudness:

$\square$ As loud as breathing $\square$ As loud as talking $\square$ Louder than talking $\square$ Very loud

3. How frequently do you snore?

$\square$ Nearly every day $\square$ 3-4 times a week $\square$ 1-2 times a week

$\square$ 1-2 times a month $\quad \square$ Never or nearly never

4. Does your snoring bother other people?

$\square$ Yes $\quad \square$ No $\quad \square$ Don't know

5. While sleeping - have you been told you have breathing pauses?

$\square$ Nearly every day $\quad \square$ 3-4 times a week $\square$ 1-2 times a week

$\square$ 1-2 times a month $\square$ Never or nearly never

6. How often do you feel tired after sleeping?
$\square$ Nearly every day
$\square$ 3-4 times a week
1-2 times a week
$\square$ 1-2 times a month
$\square$ Never or nearly never 
7. How often do you feel tired during waketime?
$\square$ Nearly every day $\square$ 3-4 times a week
1-2 times a week
口 1-2 times a month
$\square$ Never or nearly never

Please tick the most appropriate option:

8. Have you ever fallen asleep while driving?
$\square$ Yes
$\square$ No

9. Do you have high blood pressure?
$\square$ Yes
$\square$ No
$\square$ Don't know

10. Prior to this pregnancy, did you snore?
$\square$ Yes
$\square$ No
$\square$ Don't know

11. In the 6 months before becoming pregnant, had your weight changed?
$\square$ Increased
$\square$ Decreased
$\square$ No Change

Thank you! 
Appendix C

Multivariate Apnea Risk Index 


\section{MAP Index}

ID number

Date

During the last month, have you had, or have you been told about the following symptoms:

(show the frequency by putting a cross in one box)

\begin{tabular}{|c|c|c|c|c|c|}
\hline never & $\begin{array}{c}\text { rarely, } \\
\text { less than } \\
\text { once a } \\
\text { week }\end{array}$ & $\begin{array}{c}1-2 \\
\text { times a } \\
\text { week }\end{array}$ & $\begin{array}{c}3-4 \\
\text { times a } \\
\text { week }\end{array}$ & $\begin{array}{c}5-7 \\
\text { times a } \\
\text { week }\end{array}$ & $\begin{array}{c}\text { don't } \\
\text { know }\end{array}$ \\
\hline
\end{tabular}

1. snorting or gasping during sleep

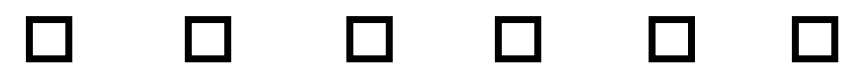

2. loud snoring during sleep

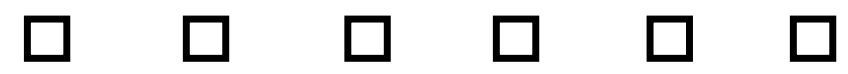

3. breathing stops, choke or struggle for breath (during sleep)

4. frequent awakenings

$\square \quad \square \quad \square \quad \square \quad \square \quad \square$

$\square \quad \square \quad \square \quad \square \quad \square \quad \square$

5. tossing, turning or thrashing while asleep

6. difficulty falling asleep

$\square \quad \square \quad \square \quad \square \quad \square \quad \square$

7. legs feel jumpy or jerky at night

$\square \quad \square \quad \square \quad \square \quad \square \quad \square$

8. morning headaches

$\begin{array}{llllll}\square & \square & \square & \square & \square\end{array}$

9. falling asleep when at work or school

$\begin{array}{llllll}\square & \square & \square & \square & \square\end{array}$

10. falling asleep when driving

$\square \quad \square \quad \square \quad \square \quad \square \quad \square$ 


\section{MAP Index}

ID number

(show the frequency by putting a cross in one box)

11. excessive sleepiness during the day

12. awaken feeling paralysed, unable to move for short period's

13. find yourself in a vivid dreamlike state when falling asleep or awakening even though you know you're awake
Date

\begin{tabular}{|c|c|c|c|c|c|}
\hline never & $\begin{array}{c}\text { rarely, } \\
\text { less than } \\
\text { once a } \\
\text { week }\end{array}$ & $\begin{array}{c}1-2 \\
\text { times a } \\
\text { week }\end{array}$ & $\begin{array}{c}3-4 \\
\text { times a } \\
\text { week }\end{array}$ & $\begin{array}{c}5-7 \\
\text { times a } \\
\text { week }\end{array}$ & $\begin{array}{c}\text { don't } \\
\text { know }\end{array}$ \\
\hline
\end{tabular}

$\square \quad \square \quad \square \quad \square \quad \square \quad \square$

$\square \quad \square \quad \square \quad \square \quad \square \quad \square$

14. falling asleep during the day, particularly when you are not busy (not including planned naps)?

15. morning fatigue (on awakening)?

16. constant unexplained fatigue?

\section{Thank you!}

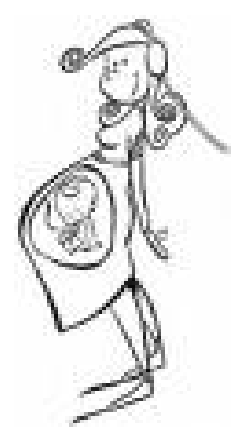


Appendix D

Restless Legs Syndrome Questionnaire 
Restless Legs Syndrome Questionnaire

Please indicate whether the following descriptions have applied to you in the past month:

1. I feel the urge to move my legs - usually because of an uncomfortable or unpleasant sensation in my legs.

(this is not due to leg cramps, positional discomfort or other medical conditions)
$\square$ Yes
$\square$ No
$\square$ Don't know

2. The urge to move my legs and the accompanying unpleasant sensations begin or worsen during periods of rest or inactivity such as lying down or sitting.
$\square$ Yes
$\square$ No
$\square$ Don't know

3. The urge to move my legs and the accompanying unpleasant sensations are partially or totally relieved by movement, such as walking or stretching.
$\square$ Yes
$\square$ No
$\square$ Don't know

4. The urge to move my legs and the accompanying unpleasant sensations only occur or are worse in the evening or night rather than during the day.
$\square$ Yes
$\square$ No
$\square$ Don't know

How often do you experience these symptoms?

$\square$ at least twice a week $\quad \square$ Less than twice a week $\quad \square$ Never

Do these symptoms cause you significant distress or impairment in important areas of functioning? i.e. sleep, energy, daily activities, mood etc.

$$
\square \text { Yes } \quad \square \text { No }
$$

If so, please describe

Did you experience these symptoms before you became pregnant?
$\square$ Yes
$\square$ No 


\section{University Library}

\section{- M I N E R VA}

\section{A gateway to Melbourne's research publications}

Minerva Access is the Institutional Repository of The University of Melbourne

\section{Author/s:}

Wilson, Danielle L.

Title:

Sleep-disordered breathing in gestational hypertension and preeclampsia: impact on maternal and fetal outcomes

Date:

2018

Persistent Link:

http://hdl.handle.net/11343/216478

\section{Terms and Conditions:}

Terms and Conditions: Copyright in works deposited in Minerva Access is retained by the copyright owner. The work may not be altered without permission from the copyright owner. Readers may only download, print and save electronic copies of whole works for their own personal non-commercial use. Any use that exceeds these limits requires permission from the copyright owner. Attribution is essential when quoting or paraphrasing from these works. 